\title{
Cleanup Verification Package for the 618-2 Burial Ground
}

\author{
Prepared for the U.S. Department of Energy \\ by Washington Closure Hanford
}

December 2006 


\section{EXECUTIVE SUMMARY}

This cleanup verification package documents completion of remedial action for the 618-2 Solid Waste Burial Ground, also referred to as Solid Waste Burial Ground No. 2; Burial Ground No. 2; 318-2; and Dry Waste Burial Site No. 2. This site is located in the 300-FF-2 Operable Unit in the 600 Area of the Hanford Site in southeastern Washington State. During its period of operation from 1951 to 1954 , the $618-2$ site was used primarily for the disposal of contaminated equipment, materials, and laboratory waste from 300 Area Facilities.

Site excavation and waste disposal of the 618-2 waste site are complete, and the exposed surfaces have been sampled and analyzed to verify attainment of the remedial action goals. Results of the sampling, laboratory analyses, and data evaluations for the 618-2 site indicate that all remedial action objectives for direct exposure, protection of groundwater, and protection of the Columbia River have been met for industrial land use (see Table ES-1).

The site meets cleanup standards and has been reclassified as "interim closed out" in accordance with the Hanford Federal Facility Agreement and Consent Order (Ecology et al. 1989) and the Waste Site Reclassification Guideline TPA-MP-14 (RL-TPA-90-0001) (DOE-RL 1998). A copy of the waste site reclassification form is included as Attachment ES-1. 
CVP-2006-00010

Rev. 0

Table ES-1. Summary of Cleanup Verification Results for the 618-2 Waste Site. (2 Pages)

\begin{tabular}{|c|c|c|c|c|}
\hline $\begin{array}{l}\text { Regulatory } \\
\text { Requirement }\end{array}$ & Remedial Action Goals & Results & $\begin{array}{l}\text { Remedial } \\
\text { Action } \\
\text { Objectives } \\
\text { Attained? }\end{array}$ & Ref. \\
\hline $\begin{array}{l}\text { Direct Exposure - } \\
\text { Radionuclides }\end{array}$ & $\begin{array}{l}\text { Attain } 15 \mathrm{mrem} / \mathrm{yr} \text { dose rate above } \\
\text { background over } 1,000 \text { years. Attain } \\
\text { the CERCLA risk range of } 10^{-4} \text { to } 10^{-6} \text {. }\end{array}$ & $\begin{array}{l}\text { Maximum dose rate calculated by RESRAD } \\
\text { for the site is } 1.19 \text { mrem/yr and } 0.212 \mathrm{mrem} / \mathrm{yr} \\
\text { for the combined staging pile and } \\
\text { decontamination area. The maximum risk } \\
\text { calculated by RESRAD for the site is } \\
1.5 \times 10^{-5} \text { and } 9.58 \times 10^{-7} \text { for the combined } \\
\text { staging pile and decontamination area. No } \\
\text { radionuclides were detected above } \\
\text { background in the overburden soil. }\end{array}$ & Yes & d \\
\hline $\begin{array}{l}\text { Direct Exposure- } \\
\text { Nonradionuclides }\end{array}$ & Attain individual COC RAGs. & $\begin{array}{l}\text { All individual COC concentrations are below } \\
\text { the RAGs. }\end{array}$ & Yes & d \\
\hline \multirow[t]{4}{*}{$\begin{array}{l}\text { Nonradionuclide } \\
\text { Risk Requirements }\end{array}$} & $\begin{array}{l}\text { Attain hazard quotient of }<1 \text { for } \\
\text { noncarcinogens. }\end{array}$ & All hazard quotients are below 1 . & \multirow{4}{*}{ Yes } & \multirow{4}{*}{ d } \\
\hline & $\begin{array}{l}\text { Attain cumulative hazard quotient of }<1 \\
\text { for noncarcinogens. }\end{array}$ & $\begin{array}{l}\text { The cumulative hazard quotients for the site, } \\
\text { the overburden, and the combined staging pile } \\
\text { and decontamination area are all below } 1 .\end{array}$ & & \\
\hline & $\begin{array}{l}\text { Attain excess cancer risk of }<1 \times 10^{-5} \\
\text { for individual carcinogens. }\end{array}$ & \multirow{2}{*}{$\begin{array}{l}\text { Excess cancer risks were not calculated } \\
\text { because all nonradionuclide carcinogenic } \\
\text { COCs (arsenic and cadmium) were detected } \\
\text { below statistical background levels. }\end{array}$} & & \\
\hline & $\begin{array}{l}\text { Attain a total excess cancer risk of }<1 \times \\
10^{-5} \text { for carcinogens. }\end{array}$ & & & \\
\hline \multirow[t]{4}{*}{$\begin{array}{l}\text { Groundwater/River } \\
\text { Protection - } \\
\text { Radionuclides }\end{array}$} & $\begin{array}{l}\text { Attain single COC groundwater and } \\
\text { river protection RAGs. }\end{array}$ & $\begin{array}{l}\text { With the exception of uranium, RESRAD } \\
\text { modeling predicts that contaminants from the } \\
\text { site and the combined staging pile and } \\
\text { decontamination area do not reach } \\
\text { groundwater within } 1,000 \text { years. Uranium is } \\
\text { predicted to reach groundwater within } 1,000 \\
\text { years, but at concentrations below the RAG. } \\
\text { Therefore, all groundwater and river protection } \\
\text { RAGs have been attained. }\end{array}$ & \multirow{4}{*}{ Yes } & \multirow{4}{*}{$d$} \\
\hline & $\begin{array}{l}\text { Attain National Primary Drinking Water } \\
\text { Standards: } 4 \mathrm{mrem} / \mathrm{yr} \text { (beta/gamma) } \\
\text { dose rate to target receptors/organs. }\end{array}$ & $\begin{array}{l}\text { No beta-/gamma-emitting radionuclides are } \\
\text { predicted to reach groundwater within } 1,000 \\
\text { years; therefore, the Drinking Water Standard } \\
\text { RAGs have been attained. }\end{array}$ & & \\
\hline & $\begin{array}{l}\text { Meet drinking water standards for } \\
\text { nonuranium alpha emitters: the more } \\
\text { stringent of the } 15 \mathrm{pCi} / \mathrm{L} \mathrm{MCL} \text { or } 1 / 25^{\text {th }} \\
\text { of the derived concentration guide per } \\
\text { DOE Order } 5400.5 .^{\mathrm{b}}\end{array}$ & $\begin{array}{l}\text { No nonuranium alpha-emitting radionuclides } \\
\text { are predicted to reach groundwater within } \\
1,000 \text { years; therefore, the drinking water } \\
\text { standards for alpha emitters have been } \\
\text { attained. }\end{array}$ & & \\
\hline & $\begin{array}{l}\text { Meet total uranium standard of } \\
21.2 \mathrm{pCi} / \mathrm{L}^{\mathrm{c}}\end{array}$ & $\begin{array}{l}\text { The maximum predicted activity for total } \\
\text { uranium in } 1,000 \text { years }(11.2 \mathrm{pCi} / \mathrm{L}) \text { is below } \\
\text { groundwater and river protection RAGs. }\end{array}$ & & \\
\hline $\begin{array}{l}\text { Groundwater/River } \\
\text { Protection - } \\
\text { Nonradionuclides }\end{array}$ & $\begin{array}{l}\text { Attain individual nonradionuclide } \\
\text { groundwater and river cleanup } \\
\text { requirements. }\end{array}$ & $\begin{array}{l}\text { Soil cleanup levels for groundwater and river } \\
\text { protection have been attained. }\end{array}$ & Yes & d \\
\hline
\end{tabular}


CVP-2006-00010

Rev. 0

\section{Table ES-1. Summary of Cleanup Verification Results for the}

618-2 Waste Site. (2 Pages)

\begin{tabular}{|c|c|c|c|c|}
\hline $\begin{array}{c}\text { Regulatory } \\
\text { Requirement }\end{array}$ & Remedial Action Goals & Results & $\begin{array}{c}\text { Remedial } \\
\text { Action } \\
\text { Objectives } \\
\text { Attained? }\end{array}$ & Ref. \\
\hline \multirow{2}{*}{$\begin{array}{l}\text { Supporting } \\
\text { Information }\end{array}$} & \multicolumn{3}{|c|}{ 1. $618-2$ cleanup verification sample location design (Appendix D) } & e \\
\hline & \multicolumn{3}{|c|}{ 2. Closeout Plan for the 618-2 Burial Ground } & $f$ \\
\hline
\end{tabular}

a "National Primary Drinking Water Regulations" (40 Code of Federal Regulations 141).

b Radiation Protection of the Public and the Environment (DOE Order 5400.5).

c Based on the isotopic distribution of uranium in the Hanford Site background, the $30 \mu \mathrm{g} / \mathrm{L}$ MCL (65 Federal Register 76708) corresponds to 21.2 $\mathrm{pCi} / \mathrm{L}$. Concentration-to-activity calculations are documented in Calculation of Total Uranium Activity Corresponding to a Maximum Contaminant Level for Total Uranium of 30 Micrograms per Liter in Groundwater, 0100X-CA-V0038 (BHI 2001).

d Cleanup Verification 95\% UCL Calculations for 618-2 Shallow Zone/Deep Zone/Overburden and 618-2, 618-3, 618-8 Staging Pile and Decon Pad Footprint, 0600X-CA-V0060, Rev. 2, Washington Closure Hanford, Richland, Washington.

e 618-2 Shallow, Deep Zone, Overburden/Stockpile Area Sampling Plan, 0300X-CA-V0077, Rev. 0, Washington Closure Hanford, Richland, Washington.

f Closeout Plan for the 618-2 Burial Ground, CCN 129577, Washington Closure Hanford, Richland, Washington (WCH 2006a).

CERCLA = Comprehensive Environmental Response, Compensation, and Liability Act of 1980

COC = contaminant of concern

$\mathrm{MCL} \quad=$ maximum contaminant level (drinking water standard)

NA = not applicable

RAG = remedial action goal

UCL = upper confidence limit 
CVP-2006-00010

Rev. 0

\section{Attachment ES-1 \\ Waste Site Reclassification Form}

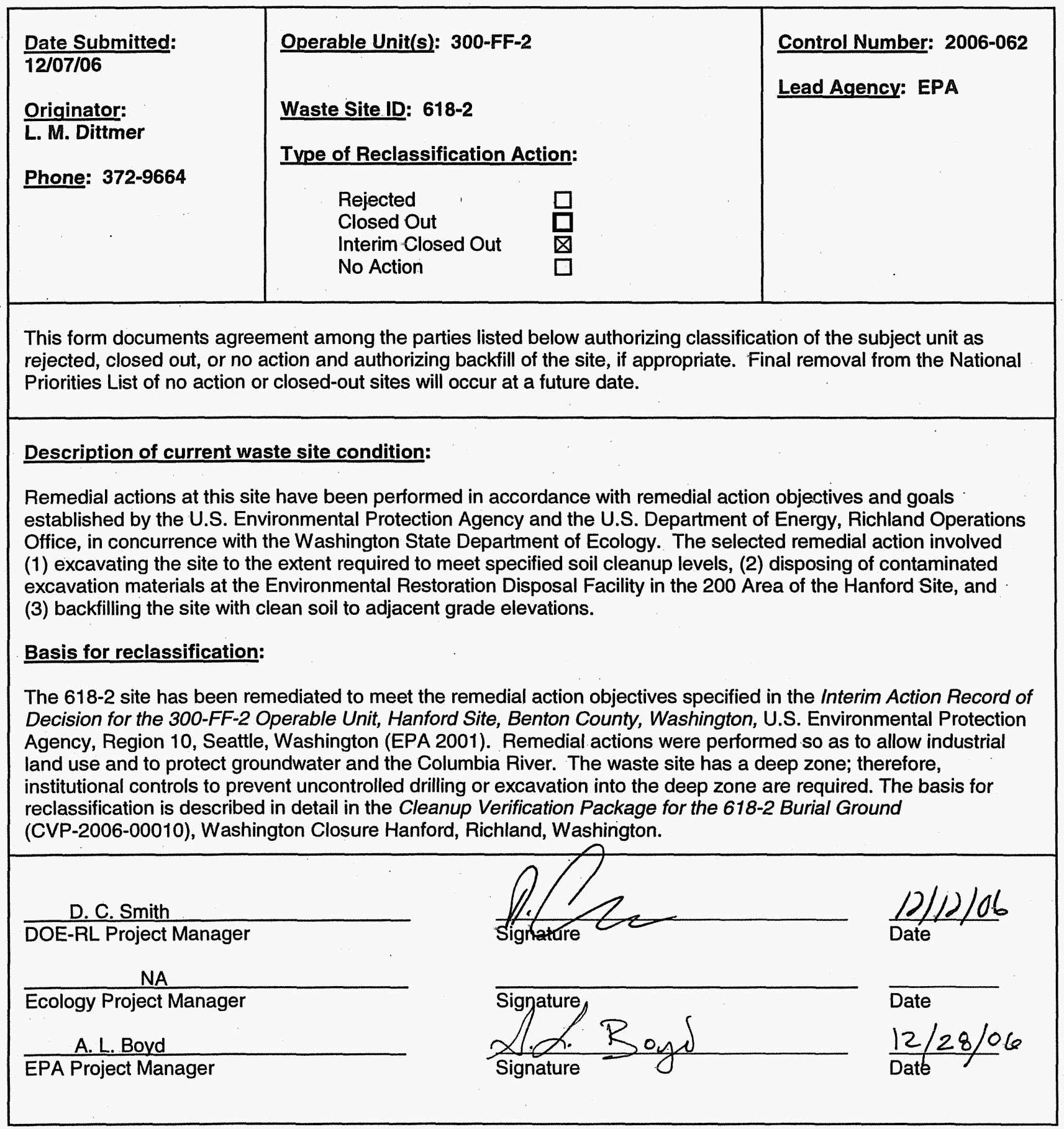




\section{CONTENTS}

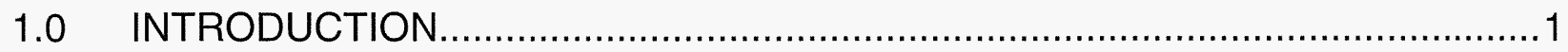

2.0 SITE DESCRIPTION AND SUPPORTING INFORMATION ..............................

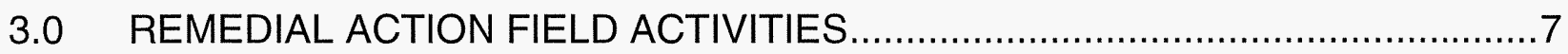

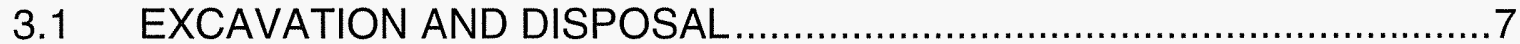

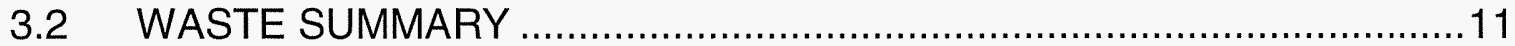

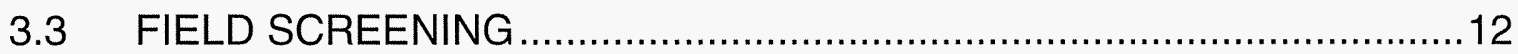

3.3.1 In-Process Radiological Surveys ............................................. 12

3.3.2 Final Status Radiological Surveys ……....................................16

3.4 BIASED SAMPLING, ANALYSIS, AND RESULTS ............................16

3.5 CLEANUP VERIFICATION SAMPLING AND ANALYSIS ....................24

4.0 CLEANUP VERIFICATION DATA EVALUATION ...........................................25

4.1 DATA QUALITY ASSESSMENT PROCESS .....................................25

4.2 CONTAMINANTS OF CONCERN 95\% UPPER CONFIDENCE LIMIT ...25

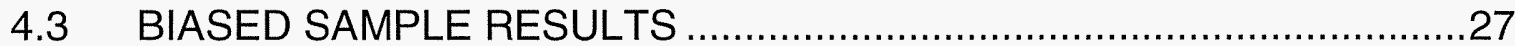

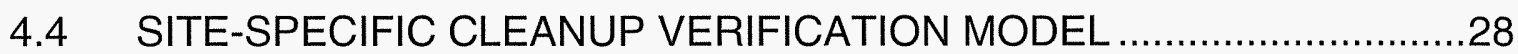

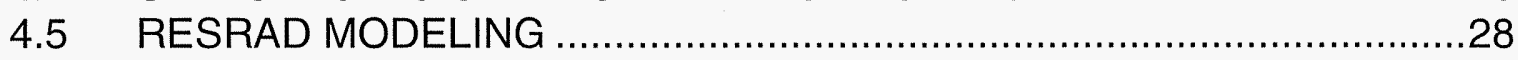

5.0 EVALUATION OF REMEDIAL ACTION GOAL ATTAINMENT …....................29

5.1 DIRECT EXPOSURE SOIL REMEDIAL ACTION GOALS ATTAINED ....29

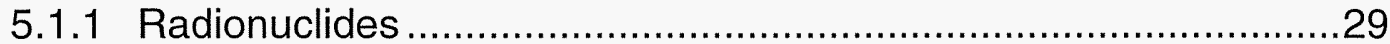

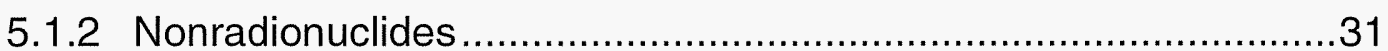

5.2 GROUNDWATER REMEDIAL ACTION GOALS ATTAINED ...................32

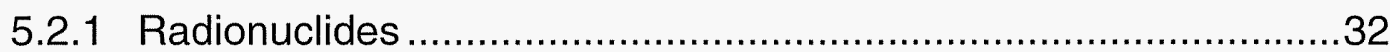

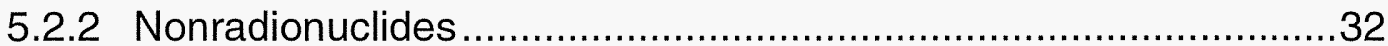

5.3 COLUMBIA RIVER REMEDIAL ACTION GOALS ATTAINED .................33

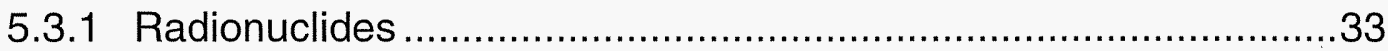

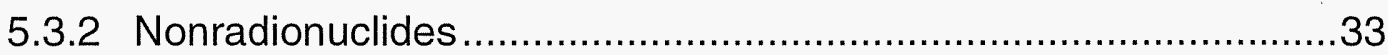

5.4 WAC 173-340 THREE-PART TEST FOR NONRADIONUCLIDES ..........33

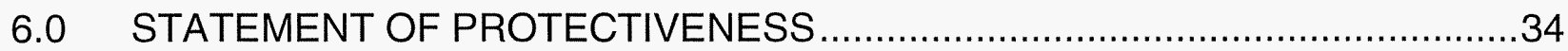

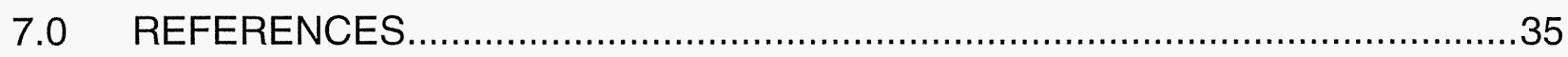




\section{APPENDICES}

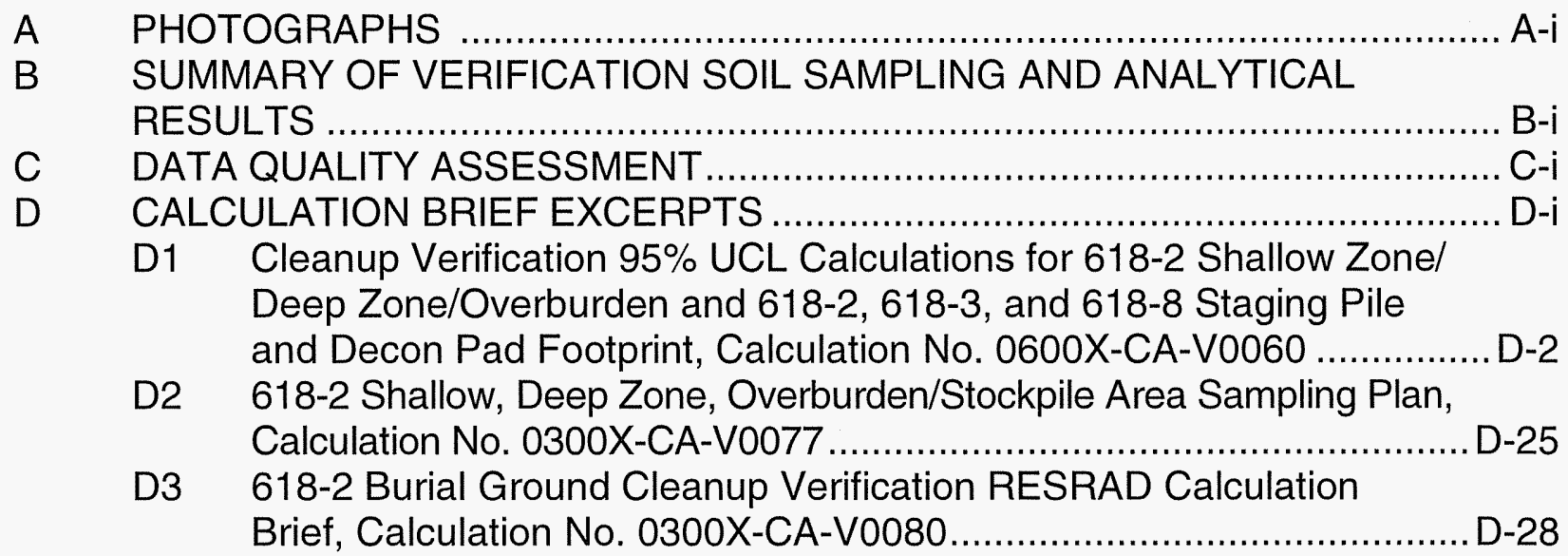

\section{FIGURES}

1. Hanford Site Map and Location of the 618-2 Waste Site. ...................................

2. Pre-Remediation Topographic Map for the 618-2 Waste Site............................

3. Post-Remediation Topographic Civil Survey Map for the 618-2 Waste Site. ........9

4. 618-2 Burial Ground Test Pit Sample Locations. ...........................................14

5. Location of Test Pit and Potholes.............................................................. 15

6. Radiological Mapping (Gamma) Survey Results for the 618-2 Site. ..................17

7. Radiological Mapping (Beta) Survey Results for the 618-2 Site........................18

8. Radiological Mapping (FIDLER) Survey Results for the 618-2 Site. ..................19

9. Radiological Mapping (FIDLER) Survey Results for the 618-2 Site. ..................20

10. Radiological Mapping (FIDLER) Survey Results for the Staging Pile Area.........21

11. Radiological Survey Locating "Hot Spots." ....................................................22

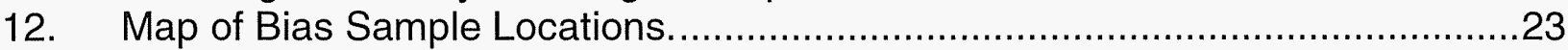

13. RESRAD Analysis - All-Radionuclides, All-Pathways Dose Rate Estimate Combined Shallow and Deep Zone...............................................................30

14. RESRAD Analysis - All-Radionuclides, All-Pathways Dose Rate Estimate Combined Staging Pile and Decontamination Pad Areas.

15. RESRAD Analysis - Radionuclide Risk, All Pathways - Combined Shallow and Deep Zone.

16. RESRAD Analysis - Radionuclide Risk - Combined Staging Pile and Decontamination Pad Areas. 


\section{TABLES}

1. Summary of Remedial Action Goals - Industrial Land Use. ..............................1

2. Summary of Remedial Action Goals - Unrestricted Land Use. ............................3

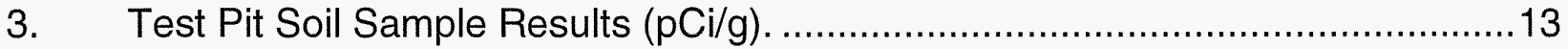

4. Number of Verification Samples for 618-2 Burial Ground. .................................24

5. Statistical Cleanup Verification Data Set. .....................................................26

6. Maximum Results from Biased Sampling. ...................................................

7. Application of the WAC 173-340 Three-Part Test..........................................34 


\section{ACRONYMS AND ABBREVIATIONS}

\begin{tabular}{|c|c|}
\hline COC & contaminant of concern \\
\hline CVP & cleanup verification package \\
\hline DQA & data quality assessment \\
\hline ELCR & excess lifetime cancer risk \\
\hline EPA & U.S. Environmental Protection Agency \\
\hline ERDF & Environmental Restoration Disposal Facility \\
\hline ESD & Explanation of Significant Differences \\
\hline GPERS & Global Positioning Environmental Radiological Surveyc \\
\hline LDR & land disposal restriction \\
\hline RAG & remedial action goal \\
\hline RAO & remedial action objective \\
\hline RDR/RAWP & remedial design report/remedial action work plan \\
\hline RESRAD & RESidual RADioactivity (dose assessment model) \\
\hline ROD & record of decision \\
\hline SAP & sampling and analysis plan \\
\hline & upper confidence limit \\
\hline & \\
\hline
\end{tabular}




\subsection{INTRODUCTION}

This cleanup verification package (CVP) documents that the 618-2 waste site was remediated in accordance with the Interim Action Record of Decision for the 300-FF-2 Operable Unit, Hanford Site, Benton County, Washington (ROD) (EPA 2001), as modified by the Explanation of Significant Differences for the 300-FF-2 Operable Unit Interim Record of Decision (ESD) (EPA 2004). Remedial action objectives (RAOs) and remedial action goals (RAGs) for this site are documented in the ROD (EPA 2001) and the Remedial Design Report/Remedial Action Work Plan for the 300 Area (RDR/RAWP) (DOE-RL 2004b). The ROD provides the U.S. Department of Energy, Richland Operations Office, the authority, guidance, and objectives to conduct this remedial action.

The remedy specified in the ROD (EPA 2001) and conducted for the 618-2 waste site included: (1) excavating the site to the extent required to meet specified soil cleanup levels, (2) disposing of contaminated excavation materials at the Environmental Restoration Disposal Facility (ERDF) in the 200 Area of the Hanford Site, and (3) backfilling the site with clean soil to the average adjacent grade elevation. Excavation was driven by RAOs for direct exposure, protection of groundwater, and protection of the Columbia River. For the respective points of compliance, RAGs, summarized in Table 1, were established for the contaminants of concern (COCs) in the RDR/RAWP (DOE-RL 2004b) to support industrial land use. Preliminary waste site contaminants of potential concern were identified in the 300 Area Remedial Action Sampling and Analysis Plan (SAP) (DOE-RL 2004a). Following excavation of the site, final COCs were identified in the Closeout Plan for the 618-2 Burial Ground (WCH 2006a) and are listed in Table 1.

Table 1. Summary of Remedial Action Goals - Industrial Land Use. (3 Pages)

\begin{tabular}{|c|c|c|c|}
\hline $\begin{array}{l}\text { Contaminants } \\
\text { of Concern }\end{array}$ & $\begin{array}{l}\text { Industrial } \\
\text { Soil Cleanup } \\
\text { Level for Direct } \\
\text { Contact } \\
\text { (mg/kg) }\end{array}$ & $\begin{array}{l}\text { Industrial Soil Cleanup Level for } \\
\text { Groundwater Protection } \\
\text { (mg/kg) }\end{array}$ & $\begin{array}{l}\text { Industrial Soil Cleanup } \\
\text { Level for River } \\
\text { Protection }^{c} \\
\text { (mg/kg) }\end{array}$ \\
\hline \multicolumn{4}{|c|}{ Nonradionuclides } \\
\hline Barium & $4,900^{d}$ & $N A^{e}$ & $N A^{e}$ \\
\hline Cadmium & $139^{d}$ & $N A^{e}$ & $N A^{e}$ \\
\hline Chromium & $>1,000,000^{\dagger}$ & $N A^{e}$ & $N A^{e}$ \\
\hline Lead & $1,000^{g}$ & $N A^{e}$ & $N A^{e}$ \\
\hline Selenium & $17,500^{h}$ & $N A^{e}$ & $N A^{e}$ \\
\hline Tin & $>1,000,000^{f}$ & $N A^{e}$ & $N A^{e}$ \\
\hline Uranium (total) & $505^{i}$ & $385^{i}$ & $385^{i}$ \\
\hline
\end{tabular}


Table 1. Summary of Remedial Action Goals - Industrial Land Use. (3 Pages)

\begin{tabular}{|c|c|c|c|}
\hline Contaminants of Concern & $\begin{array}{l}\text { Industrial Soil } \\
\text { Cleanup Level for } \\
\text { Direct Exposure } \\
\text { (pCi/g) }\end{array}$ & $\begin{array}{l}\text { Industrial Soil } \\
\text { Cleanup Level for } \\
\text { Groundwater } \\
\text { Protection }^{\mathrm{k}} \\
\text { (p/Ci/g) }\end{array}$ & $\begin{array}{l}\text { Industrial Soil Cleanup } \\
\text { Level for River } \\
\text { Protection' } \\
\text { (pCi/g) }\end{array}$ \\
\hline \multicolumn{4}{|c|}{ Radionuclides } \\
\hline Tritium (H-3) & 471 & $6,932,000$ & $13,864,000$ \\
\hline Cobalt-60 & 5.2 & $N A^{e}$ & $N A^{e}$ \\
\hline Nickel-63 & $4,026^{m}$ & $83^{m}$ & $83^{m}$ \\
\hline Strontium-90 & 2,500 & $N A^{e}$ & $\mathrm{NA}^{\mathrm{e}}$ \\
\hline Cesium-137 & 25 & $N A^{e}$ & $N A^{e}$ \\
\hline Europium-152 & 12 & $N A^{e}$ & $N A^{e}$ \\
\hline Europium-154 & 11 & $N A^{e}$ & $N A^{e}$ \\
\hline Europium-155 & 518 & $N A^{e}$ & $N A^{e}$ \\
\hline Plutonium-238 & 155 & $N A^{e}$ & $N A^{e}$ \\
\hline Plutonium-239/240 & 245 & $N A^{e}$ & $N A^{e}$ \\
\hline Plutonium-241 & $12,900^{n}$ & $N A^{e}$ & $N A^{e}$ \\
\hline Americium-241 & 210 & $N A^{e}$ & $N A^{e}$ \\
\hline Uranium-233/234 & $169^{\circ}$ & $129^{p}$ & $129^{p}$ \\
\hline Uranium-235 & $17^{\circ}$ & $13^{\mathrm{p}}$ & $13^{\mathrm{p}}$ \\
\hline Uranium-238 & $164^{\circ}$ & $125^{\mathrm{p}}$ & $125^{\mathrm{p}}$ \\
\hline Uranium (Total) & $350^{9}$ & $267^{r}$ & $267^{r}$ \\
\hline
\end{tabular}

a Direct contact values represent soil concentrations that are protective of human receptors from direct contact with contaminated waste/soil. Listed WAC 173-340-745(4), Method C cleanup standards for industrial soil apply to the top $4.6 \mathrm{~m}$ (15 ft) (WAC 173340-745[6])(1996).

b Groundwater protection values represent soil concentrations that will be protective of groundwater. Values are equal to 100 times the groundwater cleanup standard (WAC 173-340-745(4)(a)(ii)(A)) (1996), unless otherwise noted.

c River protection values represent soil concentrations that will not cause applicable river cleanup levels to be exceeded as contaminants migrate through the soil column to groundwater, and from groundwater to the river. Listed values are equal to 100 times the applicable river cleanup standard multiplied by a dilution attenuation factor of 2, unless otherwise noted.

${ }^{d}$ Cleanup limit based on the inhalation exposure pathway per WAC 173-340-750(4)(b)(ii)(A) or (B), (1996).

e The RESRAD model predicts that the constituent will not reach groundwater within 1,000 years based on a generic site profile ( $4 \mathrm{~m}[13.1 \mathrm{ft}]$ contaminated zone and $5.6 \mathrm{~m}$ [18.4 ft] uncontaminated zone) per the 300 Area RDR/RAWP, Rev. 1 (DOE/RL2004b).

f Direct contact soil cleanup levels calculated using WAC 173-340-745(4), (1996) can result in values greater than pure material (e.g., >1 million parts per million) (DOE-RL 2004b).

$\mathrm{g}$ The $1,000 \mathrm{mg} / \mathrm{kg}$ lead value reflects the Method A soil cleanup level from WAC 173-340-745 (1996).

${ }^{h}$ Method C industrial cleanup level from the Cleanup Levels and Risk Calculations (CLARC) Database (Ecology 2005).

i Based on the calculated isotopic distribution of uranium in the $300 \mathrm{Area}$ and cleanup levels of $350 \mathrm{pCi} / \mathrm{g}$ and $267 \mathrm{pCi} / \mathrm{g}$ for total uranium, the corresponding uranium concentrations are $505 \mathrm{mg} / \mathrm{kg}$ and $385 \mathrm{mg} / \mathrm{kg}$, respectively (BHI 2002) for 300-FF-2 Operable Unit sites.

i Direct exposure values represent soil activities for individual radionuclides that would meet the remedial action objective for cumulative risk (i.e., $10^{-4}$ to $10^{-6}$ risk under an industrial land-use scenario) from exposure to contaminated waste/soil. Values will be lower for multiple radionuclides to achieve the same risk endpoint. Listed values are calculated by RESRAD and apply to the top $4.6 \mathrm{~m}$ (15 ft) (DOE-RL 2004b).

k Groundwater protection values represent soil concentrations that will be protective of groundwater. Listed values are calculated by RESRAD, based on the applicable groundwater cleanup standard (DOE-RL 2004b).

1 River protection values represent soil concentrations that will not cause applicable river cleanup standards to be exceeded as contaminants migrate through the soil column to groundwater, and from groundwater to the river. Listed values are calculated by RESRAD, based on the applicable river cleanup standard (DOE-RL 2004b). 
${ }^{m}$ Cleanup level values from 100 Area RDR/RAWP (DOE-RL 2005) and (BHI 2004), 100 Area Radionuclide Lookup Values for the 1995 Interim Remedial Action Record of Decision, Calculation Number 0100X-CA-V0046, Rev. 0, Bechtel Hanford Inc. Richland, WA.

${ }^{n}$ Cleanup level value determined by RESRAD modeling for plutonium-241 using industrial land-use input parameters, as provided in the 300-FF-2 Focused Feasibility Study (DOE-RL 2000).

- Values determined using uranium equilibrium isotopic ratios and the industrial scenario selected soil cleanup level for direct exposure for total uranium $(350 \mathrm{pCi} / \mathrm{g})$ as provided in the Remedial Design Report/Remedial Action Work Plan for the 300 Area (300 Area RDR/RAWP) (DOE-RL 2004b).

$\mathrm{P}$ Values determined using uranium equilibrium isotopic ratios and the industrial scenario selected soil cleanup level for groundwater/river protection for total uranium (267 pCi/g) as provided in the 300 Area RDR/RAWP (DOE-RL 2004b).

q Listed value is equal to a $15 \mathrm{mrem} / \mathrm{yr}$ dose, based on the isotopic distribution of uranium-234, uranium-235, and uranium-238 in the 300 Area from calculation brief 0300X-CA-V0042.

$r$ Value calculated using RESRAD, based on the generic site model, with a length parallel to groundwater of $100 \mathrm{~m}(328 \mathrm{ft})$ and $\mathrm{Kd}$ values of $8.9 \mathrm{~mL} / \mathrm{g}$ for the contaminated zone and $0 \mathrm{~mL} / \mathrm{g}$ for the saturated zone (with no unsaturated, uncontaminated zone).

$\mathrm{MCL} \quad=$ maximum contamination level (drinking water standard)

NA $\quad=$ not applicable

RDR/RAWP = remedial design report/remedial action work plan

RESRAD = RESidual RADioactivity (dose assessment model)

WAC $=$ Washington Administrative Code

A waste staging pile and decontamination pad area was located in an uncontaminated area near the 618-2 site for combined use during excavation of the 618-2, 618-3 and 618-8 Burial Grounds. Verification sampling of the residual soil beneath the footprint of the 618-2, 618-3, and 618-8 Burial Ground Stockpile Staging Area is evaluated in this CVP using the RAGs for unrestricted land-use listed in Table 2 and includes the additional COCs, arsenic and silver, for the 618-3 and 618-8 waste sites. The combined waste staging pile and decontamination pad area was evaluated for cleanup verification as a single waste staging pile.

Table 2. Summary of Remedial Action Goals - Unrestricted Land Use. (2 Pages)

\begin{tabular}{|l|c|c|c|}
\hline \multicolumn{1}{|c|}{$\begin{array}{c}\text { Contaminants of } \\
\text { Concern }\end{array}$} & $\begin{array}{c}\text { Direct Exposure } \\
\text { RAGs } \\
(\mathbf{m g} / \mathbf{k g})^{\mathbf{a}}\end{array}$ & $\begin{array}{c}\text { Soil RAG for } \\
\text { Groundwater } \\
\text { Protection } \\
(\mathbf{m g} / \mathbf{k g})^{\mathbf{b}}\end{array}$ & $\begin{array}{c}\text { Soil RAG for } \\
\text { Columbia River } \\
\text { Protection } \\
(\mathbf{m g} / \mathbf{k g})^{\mathrm{c}}\end{array}$ \\
\hline Arsenic & \multicolumn{2}{|c|}{ Nonradionuclides } \\
\hline Barium & 20 & $\mathrm{NA}^{\mathrm{d}}$ & $\mathrm{NA}^{\mathrm{d}}$ \\
\hline Cadmium & 1,600 & $\mathrm{NA}^{\mathrm{d}}$ & $\mathrm{NA}^{\mathrm{d}}$ \\
\hline Chromium & $13.9^{\mathrm{e}}$ & $\mathrm{NA}^{\mathrm{d}}$ & $\mathrm{NA}^{\mathrm{d}}$ \\
\hline Lead & 120,000 & $\mathrm{NA}^{\mathrm{d}}$ & $\mathrm{NA}^{\mathrm{d}}$ \\
\hline Tin & $353^{\mathrm{f}}$ & $\mathrm{NA}^{\mathrm{d}}$ & $\mathrm{NA}^{\mathrm{d}}$ \\
\hline Selenium & 48,000 & $N A^{\mathrm{d}}$ & $1^{\mathrm{g}}$ \\
\hline Silver & 400 & $1^{\mathrm{g}}$ & $1^{\mathrm{g}}$ \\
\hline Uranium (total metal) & $400^{\mathrm{g}}$ & $8^{\mathrm{g}}$ & $\mathrm{N}^{\mathrm{h}}$ \\
\hline
\end{tabular}


CVP-2006-00010

Rev. 0

\section{Table 2. Summary of Remedial Action Goals - Unrestricted Land Use. (2 Pages)}

\begin{tabular}{|c|c|c|c|}
\hline $\begin{array}{c}\text { Contaminants of } \\
\text { Concern }\end{array}$ & $\begin{array}{c}\text { Direct Exposure } \\
\text { RAGs } \\
\text { (pCi/g) }\end{array}$ & $\begin{array}{l}\text { Soil RAG for } \\
\text { Groundwater } \\
\text { Protection } \\
\text { (pCi/g) }\end{array}$ & $\begin{array}{l}\text { Soil RAG for } \\
\text { Columbia River } \\
\text { Protection } \\
\text { (pCi } / g)\end{array}$ \\
\hline \multicolumn{4}{|c|}{ Radionuclides } \\
\hline Tritium & 711 & 30,530 & 61,060 \\
\hline Cobalt- 60 & 1.4 & $N A^{d}$ & $N A^{d}$ \\
\hline Nickel-63 & $4,026^{i}$ & $83^{i}$ & $83^{i}$ \\
\hline Strontium-90 & 4.50 & $N A^{d}$ & $N A^{d}$ \\
\hline Cesium-137 & 6.2 & $N A^{d}$ & $N A^{d}$ \\
\hline Europium-152 & 3.3 & $N A^{d}$ & $N A^{d}$ \\
\hline Europium-154 & 3.0 & $N A^{d}$ & $N A^{d}$ \\
\hline Europium-155 & 125 & $N A^{d}$ & $N A^{d}$ \\
\hline Uranium-233/234 & $27.1^{i}$ & $17.9^{\mathrm{j}}$ & 35.8 \\
\hline Uranium-235 & $2.7^{\mathrm{i}}$ & $1.8^{\mathrm{j}}$ & 3.6 \\
\hline Uranium-238 & $26.2^{i}$ & $17.3^{\mathrm{j}}$ & 34.6 \\
\hline Plutonium-238 & 38.8 & $N A^{d}$ & $N A^{d}$ \\
\hline Plutonium-239/240 & 35.1 & $N A^{d}$ & $N A^{d}$ \\
\hline Plutonium-241 & $854^{k}$ & None ${ }^{k}$ & None $^{k}$ \\
\hline Americium-241 & 32.1 & $N A^{d}$ & $N A^{d}$ \\
\hline
\end{tabular}

${ }^{a}$ Direct contact values represent soil concentrations that are protective of human receptors from direct contact with contaminated

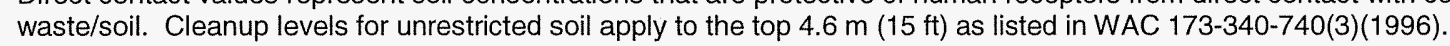

b. Groundwater protection values represent soil concentrations that will be protective of groundwater. Values are equal to 100 times the groundwater cleanup standard (WAC 173-340-740) (1996), unless otherwise noted.

c River protection values represent soil concentrations that will not cause applicable river cleanup levels to be exceeded as contaminants migrate through the soil column to groundwater, and from groundwater to the river. Listed values are equal to 100 times the applicable river cleanup standard multiplied by a dilution attenuation factor of 2 , unless otherwise noted.

d The RESRAD model predicts the constituent will not reach groundwater within 1,000 years based on a generic site profile (4.6 $\mathrm{m}$ [15 ft] contaminated zone and $6 \mathrm{~m}$ [19.6 ft] uncontaminated zone).

e Cleanup limit based on the inhalation exposure pathway per WAC 173-340-750(4)(b)(ii)(a) or (b) (1996).

f A WAC 173-340-740(3) (1996) value for lead is not available. This value is based on the Guidance Manual for the Integrated Exposure Update Biokinetic Model for Lead in Children (EPA 1994).

9 Cleanup level values from 100 Area RDR/RAWP (DOE-RL 2005) and BHI 2004, 100 Area Radionuclide Lookup Values for the 1995 Interim Remedial Action Record of Decision, Calculation Number 0100X-CA-V0046, Rev. 0, Bechtel Hanford Inc. Richland, WA.

${ }^{\mathrm{h}}$ Based on the calculated isotopic distribution of uranium in the 300 Area and a groundwater protective cleanup level of $37 \mathrm{pCi} / \mathrm{g}$ for total uranium, the corresponding uranium concentration is $53 \mathrm{mg} / \mathrm{kg}$. For direct exposure, the activity concentration corresponding to $15 \mathrm{mrem} / \mathrm{yr}$ of $56 \mathrm{pCi} / \mathrm{g}$ corresponds to a uranium concentration of $81 \mathrm{mg} / \mathrm{kg}$.

1 Cleanup level values from 100 Area RDR/RAWP (DOE-RL 2005) and BHI 2004, 100 Area Radionuclide Lookup Values for the 1995 Interim Remedial Action Record of Decision, Calculation Number 0100X-CA-V0046, Rev. 0, Bechtel Hanford Inc. Richland, WA.

I Values determined using uranium equilibrium isotopic ratios and the unrestricted land use scenario selected soil cleanup level for direct exposure for total uranium (56 pCi/g) as provided in the Remedial Design Report/Remedial Action Work Plan for the 300 Area (300 Area RDR/RAWP) (DOE-RL 2004b).

${ }^{k}$ Cleanup level values determined using RESRAD calculation with input parameters from BHI 2003, 300 Area Unrestricted LandUse Radioactive Look-up Values RESRAD Calculation, Calculation Number 0300X-CA-V0042, Rev. 0, Bechtel Hanford Inc., Richland, WA.

RAG = remedial action goal

RESRAD = RESidual RADioactivity (dose assessment model)

RDR/RAWP = remedial design report/remedial action work plan

NA $\quad$ not applicable 
Soil cleanup levels were established in the ROD based on a limited ecological risk assessment. Although not required by the ROD, a comparison against ecological risk screening levels has been made for the site COCs. Screening values were not exceeded for the waste site COCs, with the exception of selenium. Exceeding screening values does not necessarily indicate the existence of risk to ecological receptors. It is believed that the presence of selenium at this site does not pose a risk to ecological receptors because concentrations of selenium are within the range of natural site background (DOE-RL 1996). Barium, selenium, and uranium concentrations were found in soil at depths greater than $4.6 \mathrm{~m}(15 \mathrm{ft})$ and are, therefore, excluded from ecological risk consideration since the depth is below the standard point of compliance per WAC 173-340-7490(4)(b). A baseline risk assessment for the river corridor portion of the Hanford Site began in 2004 and includes a more complete quantitative ecological risk assessment. That baseline risk assessment will be used as part of the final ROD for these sites.

\subsection{SITE DESCRIPTION AND SUPPORTING INFORMATION}

The 618-2 Burial Ground, part of the 300-FF-2 Operable Unit, is located just north of the 300 Area exclusion area fence, and immediately adjacent to the 618-3 Burial Ground in the vicinity of Washington State Plane coordinates E594020, N116360 (Figure 1). The waste site, also known as Solid Waste Burial Ground No. 2; Burial Ground No. 2; 318-2; and Dry Waste Burial Site No. 2, consists of three east-west oriented trenches. A ground penetrating radar investigation, performed in 1995, identified three distinct trenches as follows: a northern trench, approximately $49 \mathrm{~m}$ (160 ft) long and $9 \mathrm{~m}$ (30 ft) wide; a middle trench approximately $54 \mathrm{~m}(175 \mathrm{ft})$ long and $15 \mathrm{~m}(50 \mathrm{ft})$ wide; and a southern trench approximately $55 \mathrm{~m}(180 \mathrm{ft})$ long and $15 \mathrm{~m}(50 \mathrm{ft})$ wide. Each trench was assumed to be approximately $4.6 \mathrm{~m}(15 \mathrm{ft})$ deep.

The 618-2 Burial Ground is reported to have been in operation from 1951 to 1954 , primarily for the disposal of contaminated equipment, materials, and laboratory waste from the 300 Area Facilities. Radiological contaminants were reported to include uranium, plutonium, and mixed fission products. The uranium waste was reportedly solid metal (uranium oxide) cuttings from reactor fuel fabrication facilities in the 300 Area. The plutonium and fission products came from waste generated in the 300 Area laboratory facilities. Non-radiological contaminants are reported to include lead and tin from various "dip-canning" processes. In 1954, a series of fires in the burial ground reportedly destroyed most of the flammable material contained in the burial ground at the time of the fires. A fire on February 17, 1954, in the northwest corner of burial trench \#2, was reported not to have spread to the southeast portion of the trench where high-activity waste was known to be concentrated (GE 1954). In 1989, during interim stabilization, a large number of automotive batteries were discovered on the surface of the waste site. The batteries were left in-place and covered with approximately $0.6 \mathrm{~m}$ $(2 \mathrm{ft})$ of clean backfill. 
Figure 1. Hanford Site Map and Location of the 618-2 Waste Site.

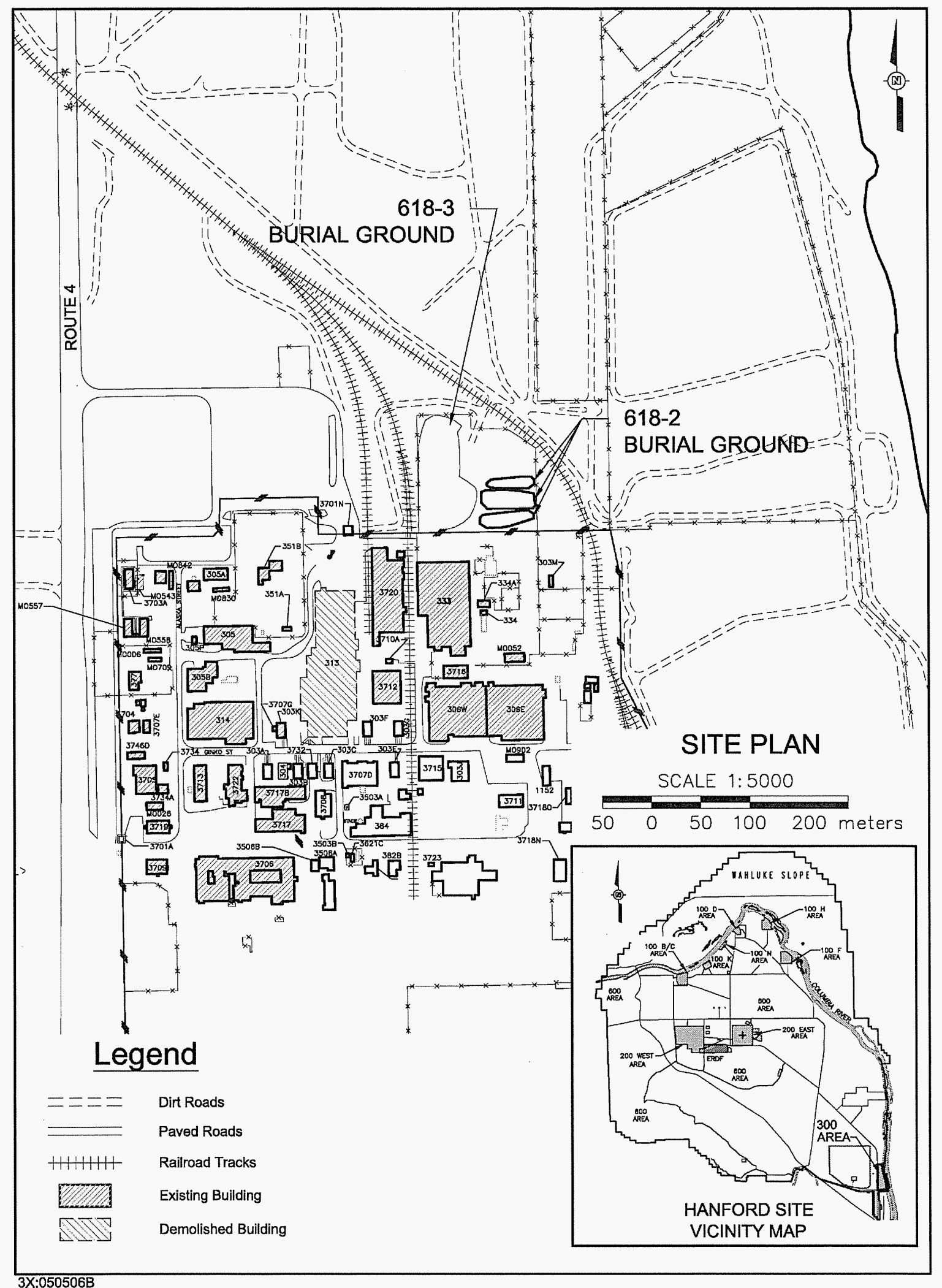




\subsection{REMEDIAL ACTION FIELD ACTIVITIES}

\subsection{EXCAVATION AND DISPOSAL}

Remedial action activities at the 618-2 waste site initially began in November 2004 and were completed in August 2006 (WCH 2006a, 2006b). Excavation and load-out operations were suspended from December 2004 to December 2005, due to the discovery of higher than anticipated levels of plutonium-contaminated waste. Remediation activities were conducted in accordance with the RDR/RAWP (DOE-RL 2004b). Remediation involved excavation and removal of buried waste and contaminated soil to the extent required to satisfy the RAOs and corresponding RAGs. In-process radiological field screening was conducted during the site remedial actions to guide the excavation and quickly assess the presence and level of contamination. The radiological survey results indicated the presence of isotopes of uranium and plutonium, as well as cesium-137 (WCH 2006a).

The buried waste consisted primarily of a wide variety of laboratory and constructiontype debris. Excavation activities found no indication of bulk liquid waste disposal at this waste site. During the initial excavation phase, contaminated soil and debris were excavated from the burial ground and transported to a designated "stockpile" area for sorting and sampling. Sorting and sampling of the excavated soil and debris was performed in a designated staging pile area. This staging pile area supported the 618-2, 618-3, and 618-8 Burial Ground excavations and is included with the closure of the 618-2 waste site. A photo of the staging pile area is provided in Appendix A. Land disposal restriction materials, primarily consisting of lead solids, were identified and separated from the bulk soil and debris during excavation and sorting operations (WCH 2006a). All excavated materials were disposed of at ERDF. Approximately 71,203 metric tons (78,488 U.S. tons) of material from the site was removed and disposed of at ERDF (WCH 2006a). Pre- and post-remediation topographic civil survey results for the 618-2 waste site are depicted in Figures 2 and 3, respectively.

Excavated material consisted of contaminated soil and a wide variety of miscellaneous debris. Some Land Disposal Restriction (LDR) materials, (primarily lead solids) were identified among the debris and were subsequently segregated from the bulk soil and non-LDR debris, then designated for separate load-out as LDR material. Subsequent to sorting, the remaining bulk soil and non-LDR debris material stockpiles were sampled to ensure that the material was in compliance with LDR restrictions. Upon receipt of sample results confirming compliance with LDR restrictions, the "released" stockpile material was loaded into ERDF containers and transported to ERDF for disposal. LDR materials that had been segregated for treatment were subsequently loaded-out and transported to ERDF under a separate waste profile. 
CVP-2006-00010

Rev. 0

Figure 2. Pre-Remediation Topographic Map for the 618-2 Waste Site.

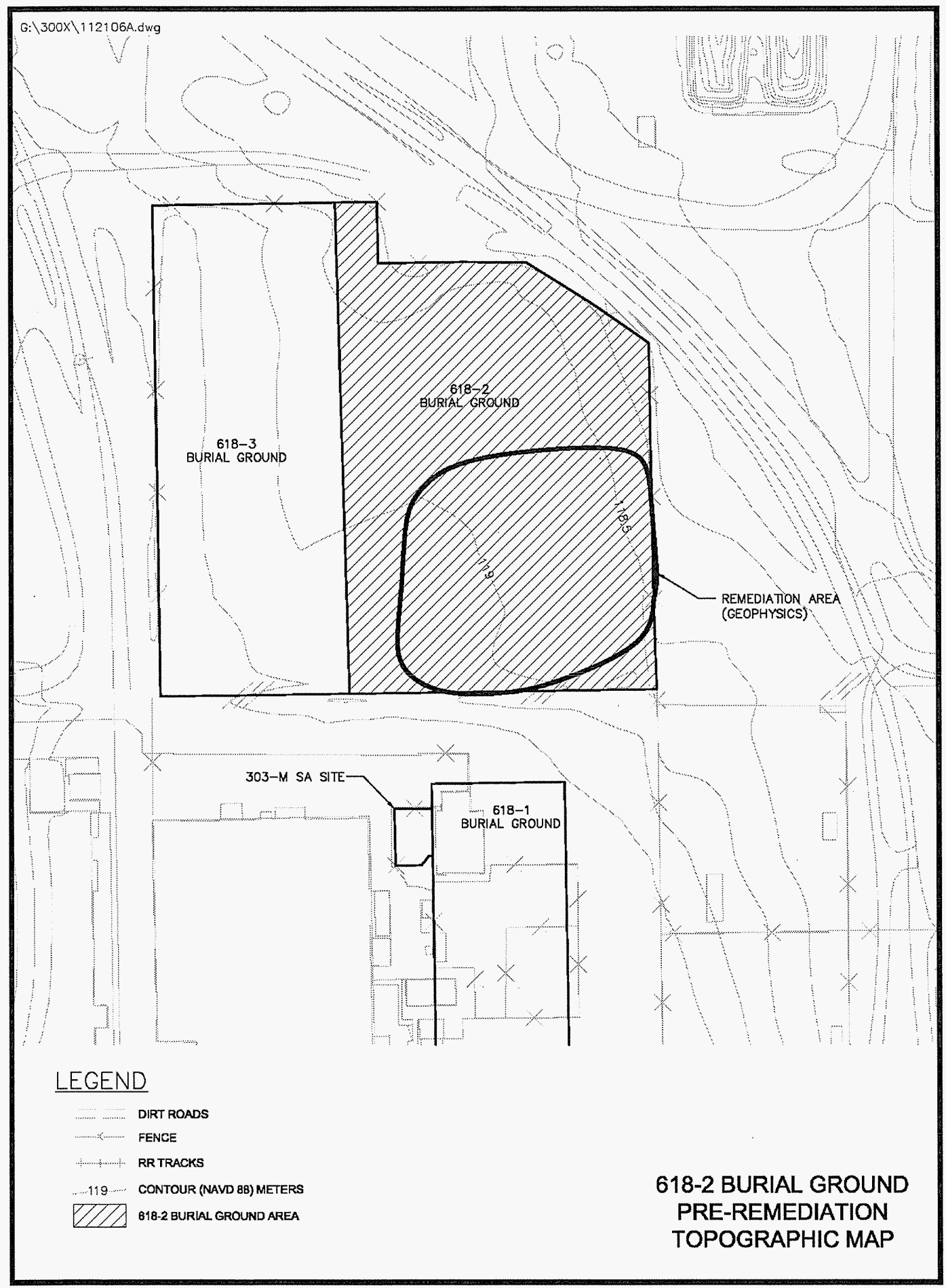


Figure 3. Post-Remediation Topographic Civil Survey Map for the 618-2 Waste Site.

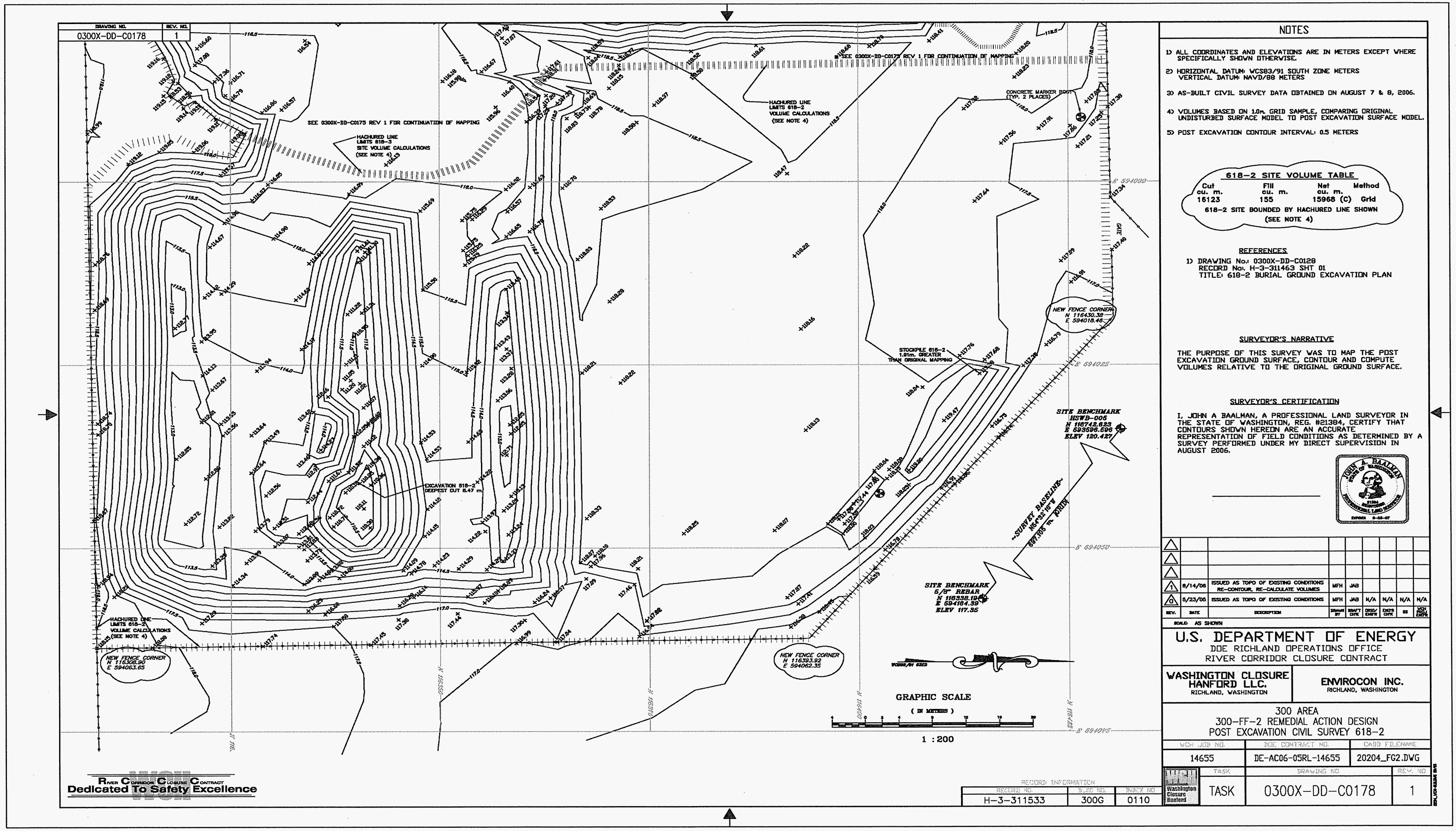


Excavation of contaminated soil and debris was performed until native soil was reached at the floor of each trench. The determination of native soil was based on the absence of debris materials, plus radiological surveys and visual observations of the soil at the base of the excavation.

When operations resumed in December 2005, the first materials designated for load-out were the existing sorted stockpiles located on the east side of the designated stockpilesorting area. Following a sampling campaign to ensure compliance with LDR restrictions, these sorted stockpiles were loaded into ERDF containers and transported to ERDF for disposal. Concurrent with the load-out of the existing sorted stockpile material, additional stockpiles of previously unsorted and unsampled 618-2 material, located on the west side of the designated stockpile/sorting area, were thoroughly sorted and subsequently sampled. Upon receipt of sample results confirming compliance with LDR restrictions, these stockpiles were also loaded into ERDF containers and transported to ERDF for disposal.

In January 2006, excavation and sorting operations resumed at the 618-2 Burial Ground trenches utilizing a revised material handling strategy that eliminated the requirement for hauling the excavated material to a designated stockpile area. The revised strategy provided a mechanism for direct sorting and stockpiling of the excavated material inside the boundaries of the existing trenches. After sorting, the "in-trench" stockpiles were then sampled to ensure compliance with LDR restrictions. Upon receipt of sample results confirming compliance with LDR restrictions, the "in-trench" stockpiles were loaded into ERDF containers and transported to ERDF for disposal. Load-out of all 618-2 "in-trench" stockpile material was completed in August 2006.

\subsection{WASTE SUMMARY}

Waste encountered during excavation of the 618-2 Burial Ground trenches was consistent with the types of material anticipated based on reported use of the 618-2 Burial Ground for disposal of contaminated equipment, building materials, and laboratory waste from the 300 Area Facilities. The waste consisted mostly of contaminated soil and debris, with some LDR material. Small quantities of laboratory glassware, drummed oil, metal crucibles and centrifuges were unearthed in discrete areas of the burial ground. A "safe" containing laboratory material was discovered during excavation of the south trench in December 2004. Characterization of the contents of the safe, including sampling and laboratory analysis of the containers found inside the safe, is still in process. LDR material, which included the pile of abandoned automotive batteries, as well as any items containing lead and/or asbestos, were set aside and loaded-out in separate load-out campaigns, under a separate waste shipping profile for LDR materials. No evidence of bulk liquid waste disposal was observed during excavation of this waste site. Photos of waste items are provided in Appendix A. 
The total amount of non-LDR waste that was transported to ERDF from the 618-2 Burial Ground waste site was approximately 71,049 metric tons (78,318 U.S. tons) as weighed across the 300 Area truck scale, in 4,181 roll-off containers. The total amount of LDR material (including the pile of abandoned automotive batteries) was approximately 154 metric tons (170 U.S. tons), in 11 roll-off containers.

\subsection{FIELD SCREENING}

Radiological surveys were performed during the site remedial actions to guide the excavation and after the excavation operations were substantially complete to provide an initial assessment of attainment of radiological cleanup levels. The surveys were performed using Global Positioning Environmental Radiological Surveyor (GPERS) with instrumentation specific to detection of radiation associated with gamma, beta, and alpha emitting radionuclides. A sodium iodide detector was used for gamma radiation and a plastic scintillation detector (SHP-380AB) was used for measurement of beta radiological activity. The plastic scintillation detector was used in addition to the sodium iodide detector because it responds principally to the beta radiation emitted by the uranium-238 progeny and is, therefore, a good indicator of residual uranium contamination. Due to the presence of residual plutonium contamination, a field instrument for the detection of low-energy radiation (FIDLER) was deployed as it responds well to low-energy gamma radiation emitted by plutonium-241 progeny.

\subsubsection{In-Process Radiological Surveys}

In-process radiological field screening was conducted during the site remedial actions to guide the excavation and quickly assess the presence and level of contamination. Contamination identified by the radiological surveys was further excavated and the site was resurveyed and sampled as necessary to verify the adequacy of removal of contaminated soil.

In June 2006, an area of yellow to orange discolored soil exhibiting high plutonium contamination was located and sampled in the eastern portion of the middle trench at a depth of $8 \mathrm{~m}(26.2 \mathrm{ft})$ below grade (111 $\mathrm{m}$ [463.2 ft] elevation). This location likely corresponds with the "high activity" waste disposal area of the middle trench that is discussed in the historical memo (GE 1954) concerning the February 17, 1954 fire at the $618-2$ Burial Ground. An additional $1 \mathrm{~m}(3.3 \mathrm{ft})$ of soil was removed from this area and a soil sample collected at a depth of $9 \mathrm{~m}(29.5 \mathrm{ft})$ below grade. The sample results indicated residual plutonium activities decreasing with depth, but not at a rate that would support an evaluation of protection of groundwater using the "50-50" model as discussed in the RDR/RAWP (DOE-RL 2004b). Additionally, a review of historical groundwater levels in the vicinity of the 618-2 site indicated a groundwater low of $104 \mathrm{~m}$ (341.2 ft) above sea level (15 m [49.2 ft] below grade) and a groundwater high of $107.5 \mathrm{~m}$ (352.7 ft) above sea level ( $11.5 \mathrm{~m} \mathrm{[37.7]} \mathrm{below} \mathrm{grade).} \mathrm{Considering} \mathrm{this}$ information, and in agreement with the EPA, a test pit was excavated to groundwater in August 2006 to remove the contaminated soil and to collect discrete soil samples for laboratory analysis to support further evaluation of protection of groundwater. A grab 
sample of water at the base of the test pit was also collected. An additional $70 \mathrm{BCM}$ (bank cubic meter) of soil was removed at this location and shipped to ERDF.

Figure 4 is a schematic of the test pit excavation and associated sample collection intervals. The results of the test pit excavation sampling (Table 3 ) indicate plutonium contamination was mobilized in the soil column at this location due to the presence of acid in the waste. The contamination decreased with depth to groundwater, but was still present at low activities in the soil column within the range of the historical groundwater table fluctuation (104.5 m [342.8 ft] to $107.5 \mathrm{~m}$ [352.7 ft]). A grab sample of water was collected for laboratory analysis and indicated the presence of contamination potentially exceeding drinking water standards. This data, however, is suspect due to the method of collection where stagnant water that had infiltrated into the excavation at the capillary fringe was collected using a backhoe bucket. The Hanford Groundwater Project will perform further evaluation of the groundwater at the 618-2 site. The results of the soil samples collected at this location for plutonium, uranium, and americium-241, including the $\mathrm{pH}$ measurements, are summarized in Table 3 with the complete laboratory analysis provided in Appendix B.

Table 3. Test Pit Soil Sample Results (pCi/g).

\begin{tabular}{|c|c|c|c|c|c|c|c|c|c|c|}
\hline $\begin{array}{c}\text { Sample } \\
\text { Number }\end{array}$ & $\begin{array}{c}\text { Sample } \\
\text { Date }\end{array}$ & $\begin{array}{c}\text { Sample } \\
\text { Elevation } \\
(\mathbf{m})\end{array}$ & $\mathrm{pH}$ & $\begin{array}{c}\text { Am-241 } \\
(\mathbf{p C i} / \mathbf{g})\end{array}$ & $\begin{array}{c}\text { Pu-238 } \\
(\mathbf{p C i} / \mathbf{g})\end{array}$ & $\begin{array}{c}\text { Pu-239/240 } \\
(\mathbf{p C i} / \mathbf{g})\end{array}$ & $\begin{array}{c}\text { Pu-241 } \\
(\mathbf{p C i} / \mathbf{g})\end{array}$ & $\begin{array}{c}\text { U-233/234 } \\
(\mathbf{p C i} / \mathbf{g})\end{array}$ & $\begin{array}{c}\text { U-235 } \\
(\mathbf{p C i} / \mathbf{g})\end{array}$ & $\begin{array}{c}\text { U-238 } \\
(\mathbf{p C i} / \mathbf{g})\end{array}$ \\
\hline $\mathrm{J} 12 \mathrm{NK} 9$ & $6 / 19 / 06$ & 111 & $\mathrm{NA}$ & 874 & 163 & 13,500 & 6,760 & 19 & 2.71 & 10.1 \\
\hline $\mathrm{J} 12 \mathrm{NL3}$ & $7 / 12 / 06$ & 110.5 & $\mathrm{NA}$ & 295 & 38.7 & 2,350 & 1,160 & 15.1 & 0.468 & 12.8 \\
\hline $\mathrm{J} 12 \mathrm{Y} 08$ & $8 / 2 / 06$ & 110 & 6.08 & 332 & 1.47 & 57.2 & 23.5 & 56 & 3.45 & 50.9 \\
\hline $\mathrm{J} 12 \mathrm{Y} 09$ & $8 / 2 / 06$ & 109 & 3.66 & 306 & 21.1 & 1,710 & 776 & 13.9 & 1.16 & 14.4 \\
\hline $\mathrm{J} 12 \mathrm{Y} 10$ & $8 / 2 / 06$ & 108 & 6.9 & 111 & 2.39 & 150 & 67.2 & 16.1 & 0.996 & 15.2 \\
\hline $\mathrm{J} 12 \mathrm{Y} 11$ & $8 / 2 / 06$ & 107 & 5.94 & 63.6 & 0.827 & 62.1 & 32.7 & 18.2 & 1.32 & 18.1 \\
\hline $\mathrm{J} 134 \mathrm{~V} 2$ & $8 / 2 / 06$ & 106 & 7.35 & 0.732 & 0.49 & 5.62 & 13.4 & 4.46 & 0.282 & 3.79 \\
\hline $\mathrm{J} 134 \mathrm{V3}$ & $8 / 2 / 06$ & 105 & 7.46 & 2.81 & 0 & 0.918 & 1.15 & 3.33 & 0.117 & 2.71 \\
\hline
\end{tabular}

Four additional potholes were dug surrounding the test pit area (see Figure 5), to assess the adequacy of removal of the plutonium contamination. Each pothole was dug $2 \mathrm{~m}(6.6 \mathrm{ft})(108 \mathrm{~m}$ [354.3 ft] elevation) and then a discrete soil sample was collected. The pothole sampling results found residual contamination and soil discoloration in the area southwest of the original test pit excavation. Therefore, additional excavation was required. After this additional soil removal, two bias samples were collected, as discussed in Section 3.4, to verify adequacy of soil remediation. The results of the bias sampling are provided in Appendix B. Figure 5 shows the location of the test pit, the four additional potholes, and the approximate boundary of additional soil removal. 
Figure 4. 618-2 Burial Ground Test Pit Sample Locations.

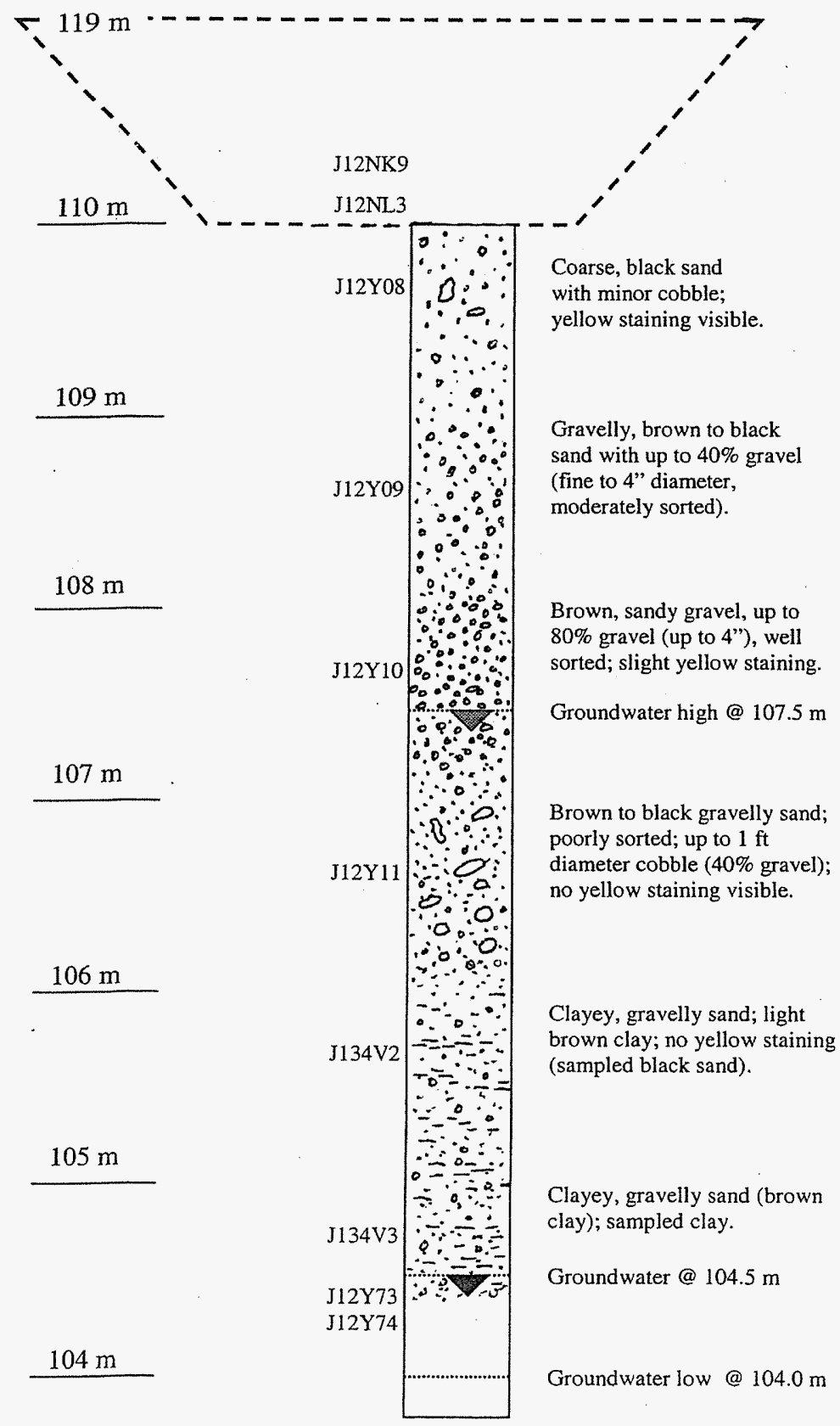

NOT TO SCALE 


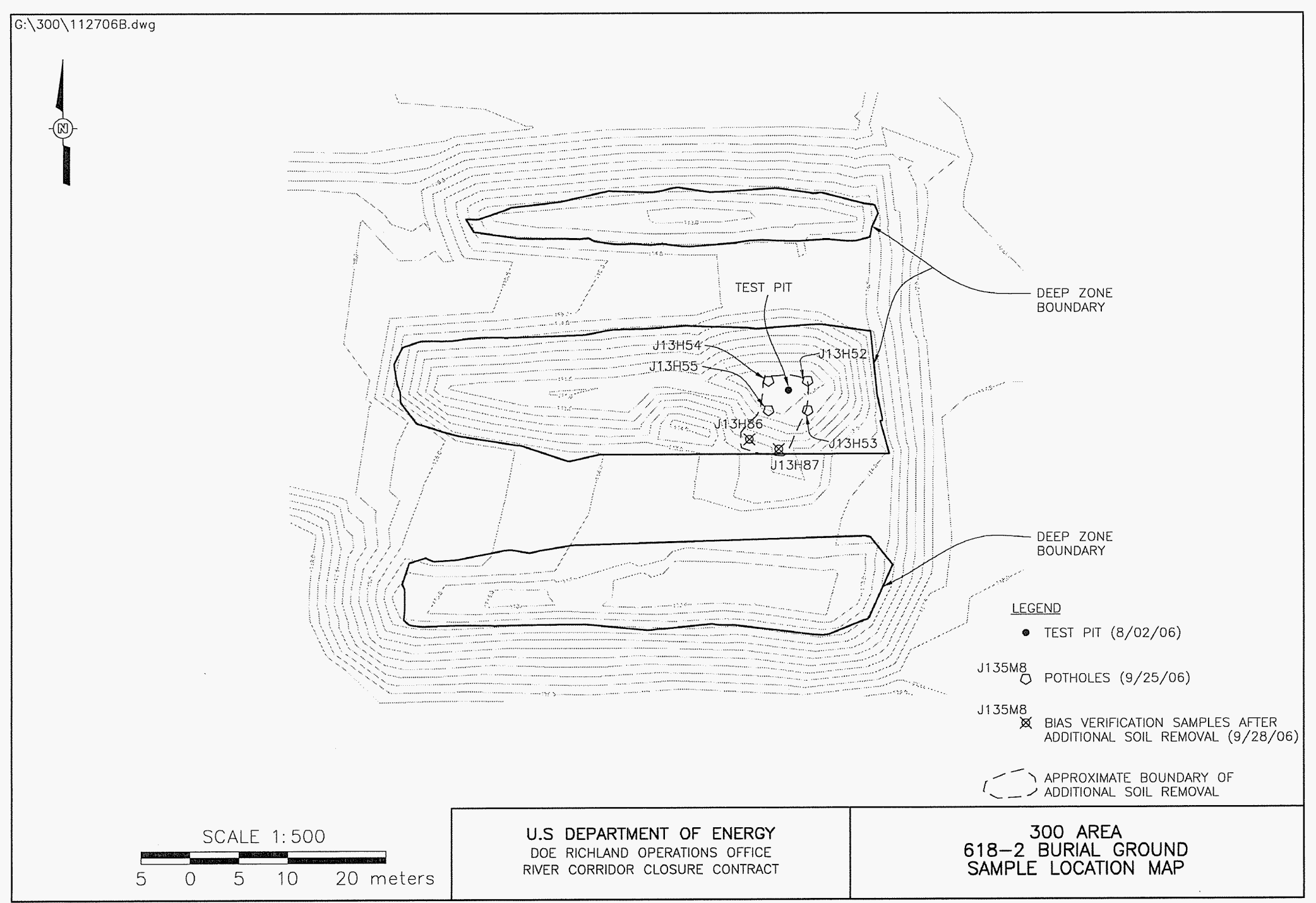

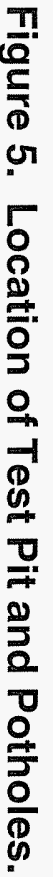




\subsubsection{Final Status Radiological Surveys}

Radiological surveys were performed in August and September of 2006, after excavation operations were substantially complete at the 618-2 waste site. These surveys were performed to provide an initial assessment of attainment of radiological cleanup levels ( $\mathrm{WCH} 2006 \mathrm{a})$. The results of the gamma and beta radiological surveys are provided in Figures 6 and 7, respectively. Figures 8 and 9 show the low energy gamma (FIDLER) surveys for the excavation.

Figure 10 provides the low-energy gamma survey results for the staging pile area. Evaluation of the combined survey results for the excavation identified several "hot spot" areas of residual radiological contamination, shown in Figure 11, that were further characterized by collecting "hot spot" samples to support waste designation prior to performing additional soil removal. The location of these samples is shown in Figure 11 and the sample results are provided in Appendix B. An additional 205 BCM of contaminated soil was removed for disposal to ERDF. Bias closeout samples were then collected to verify adequacy of the soil removal as discussed in Section 3.4.

\subsection{BIASED SAMPLING, ANALYSIS, AND RESULTS}

As provided for in the SAP (DOE-RL 2004a), biased samples were collected to provide confidence for the absence of "hot spots" in residual soil beneath locations that had visual stains, buried liquid wastes, large inventories of hazardous wastes (e.g., lead bricks), or areas where characterization or radiological survey results showed elevated contamination levels. Nine discrete soil verification soil samples (i.e., bias samples) were collected, in addition to the statistical cleanup verification samples, based on agreement with the U.S. Environmental Protection Agency (EPA) and the Department of Energy to assess area-specific residual contamination within the 618-2 Burial Ground excavation. Figure 12 provides the location of the bias soil samples.

Five of the bias samples were determined by post-excavation radiological surveys, which identified suspect "hot spots" that were further excavated (Figure 11). Of these "hot spots," three located in the northern trench were predominantly cesium-137 and were evaluated after additional excavation using radiological field screening instrumentation and one was a piece of debris that was picked up. The bias sample results for the five "hot spots" that exhibited residual plutonium are provided in Appendix B.

Two bias samples were collected at locations where anomalous waste items were found. One bias sample (Bias \#1) was collected from the floor of the excavation beneath the location where "the safe" was found. The second bias sample (Bias \#2) was collected from the floor of the excavation beneath the former location of several waste oil drums. In addition, two bias soil samples were collected adjacent to the test pit in an area where stained soil had been removed. The results of the bias verification soil samples are provided in Appendix B. 


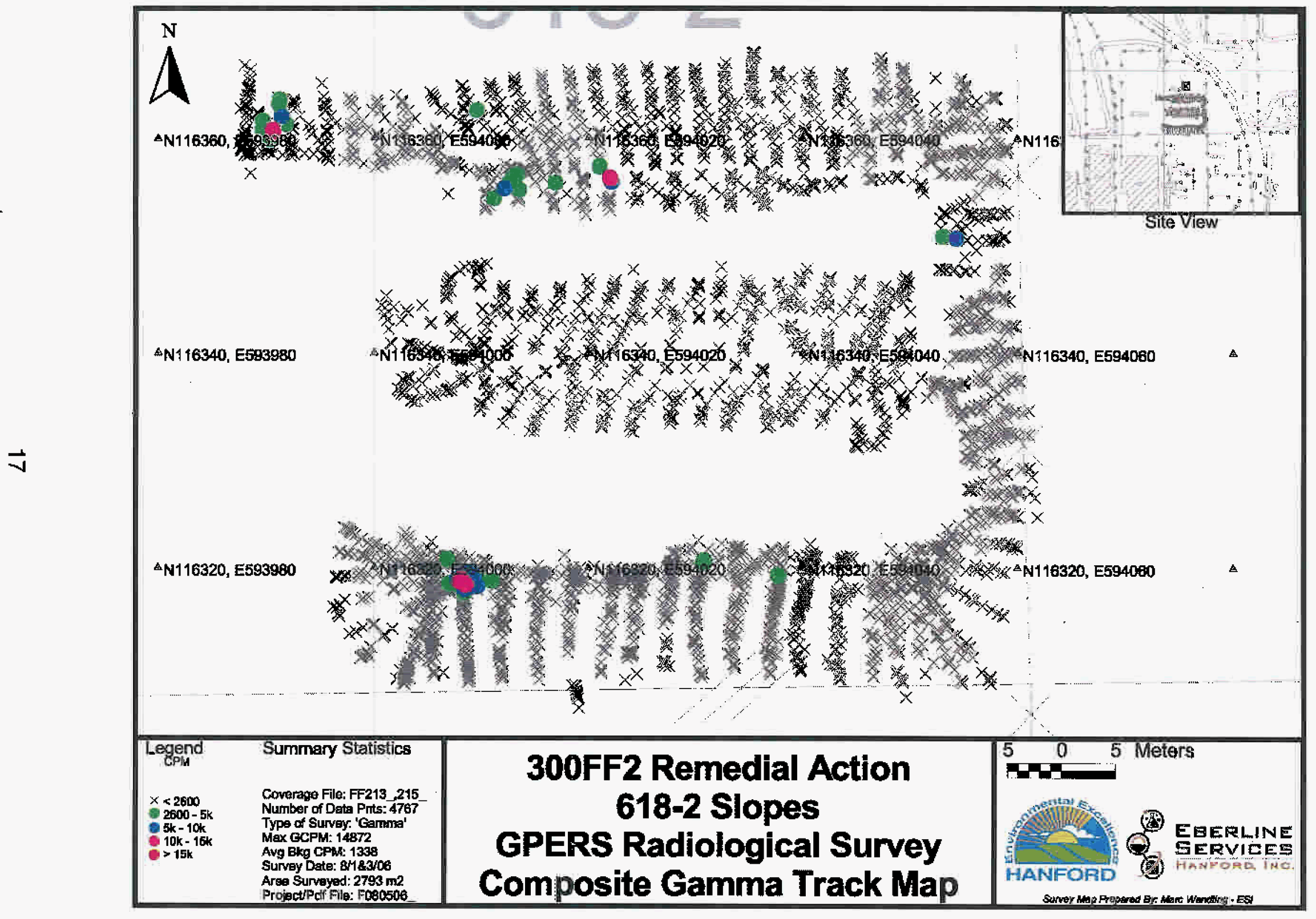

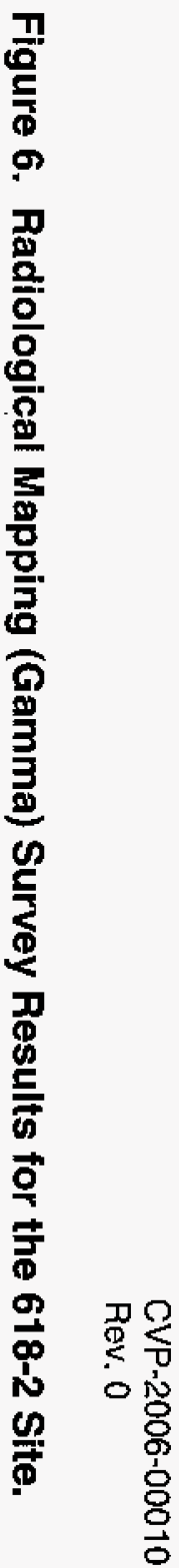




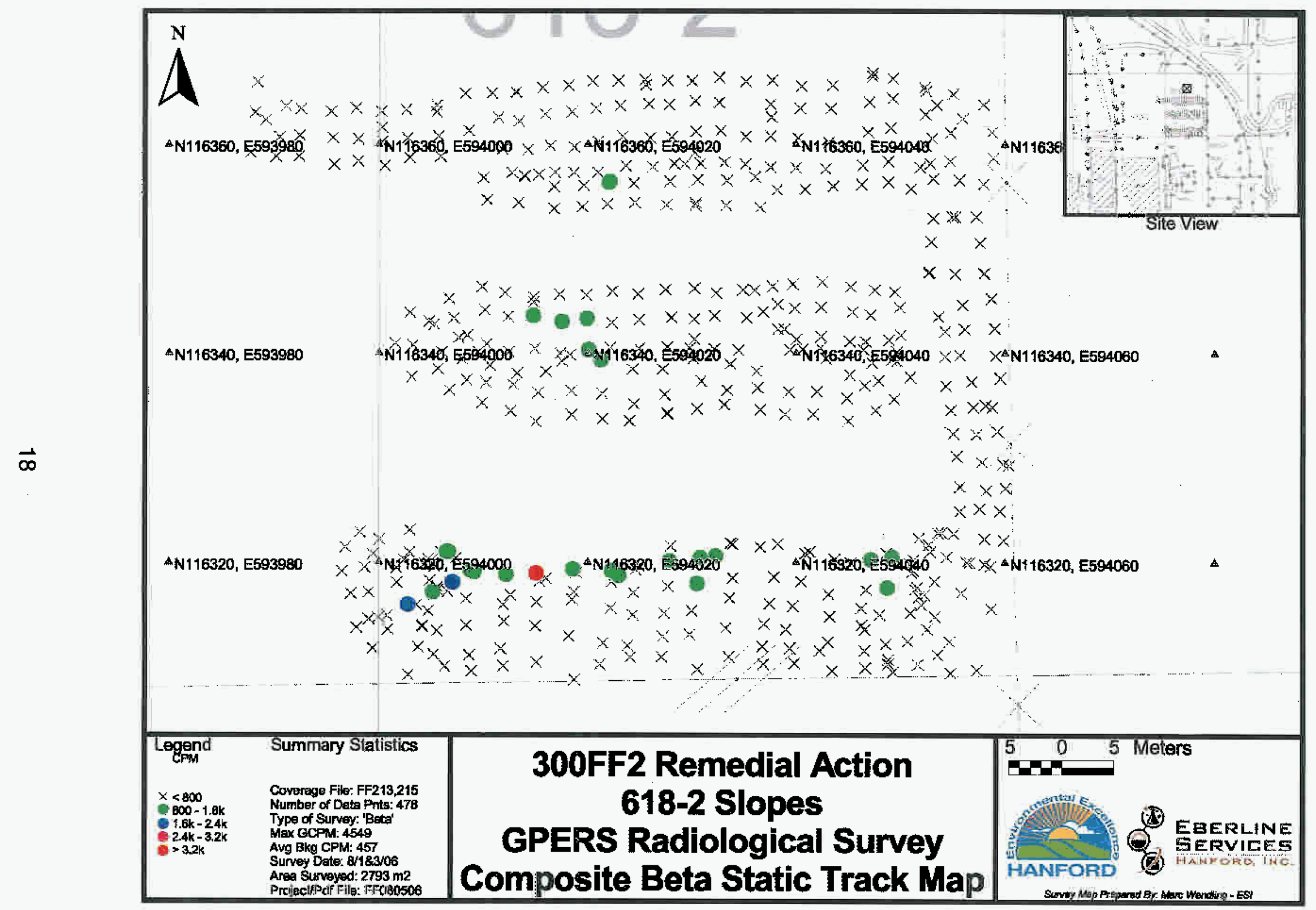

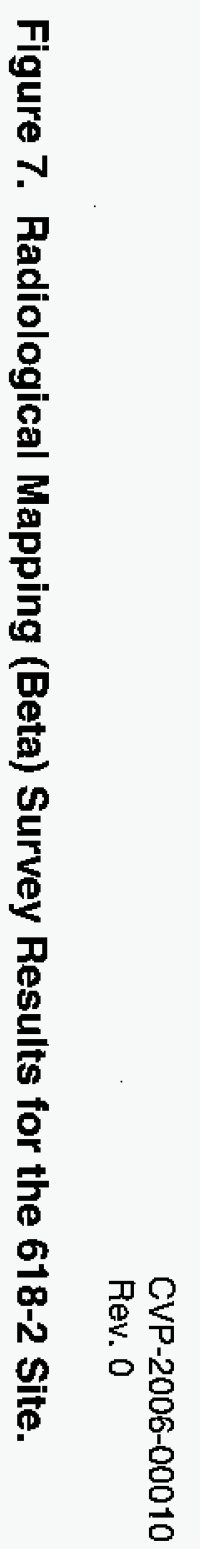




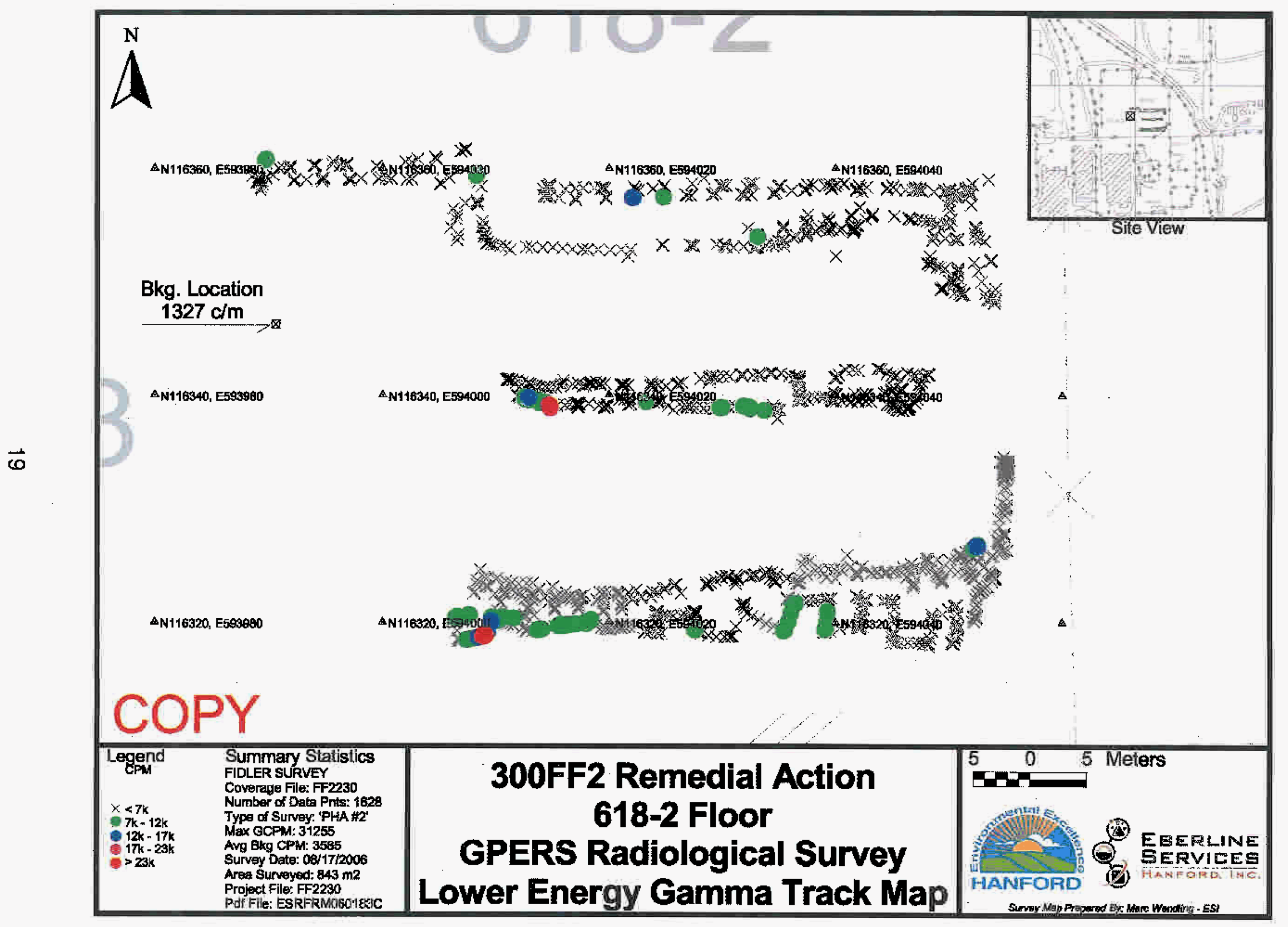

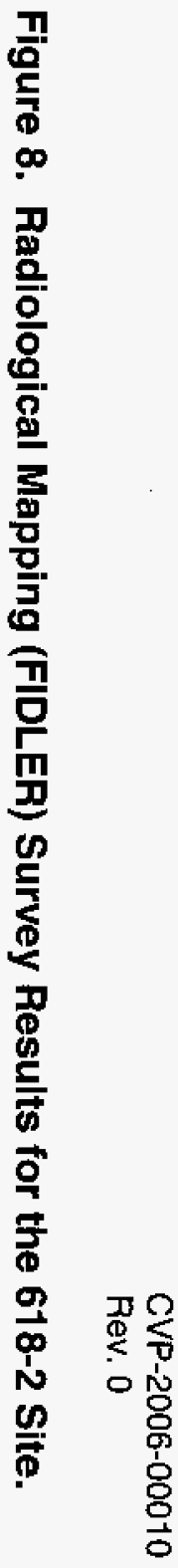




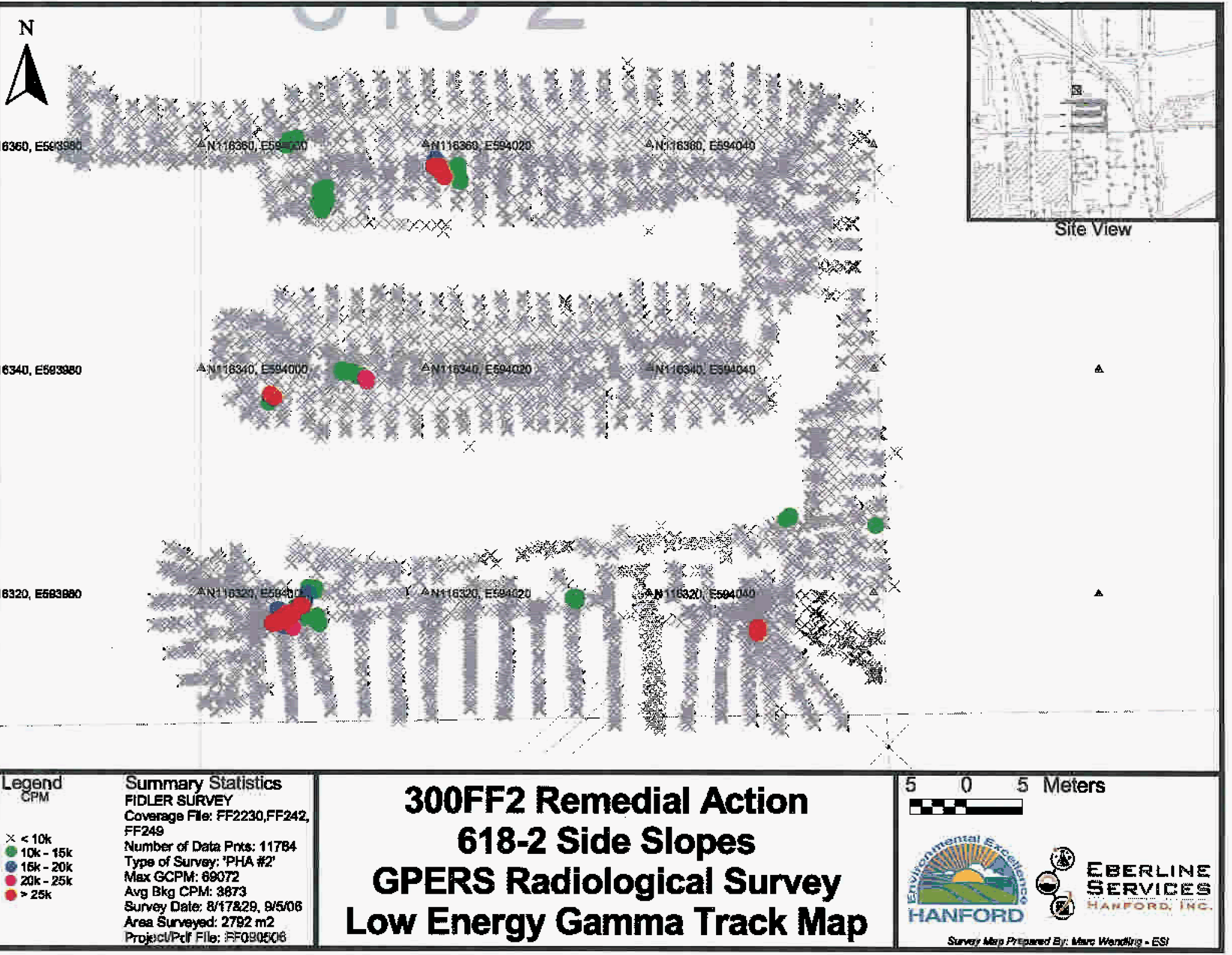

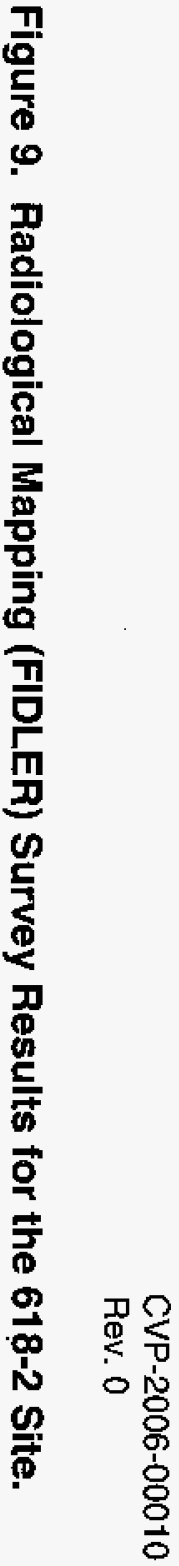




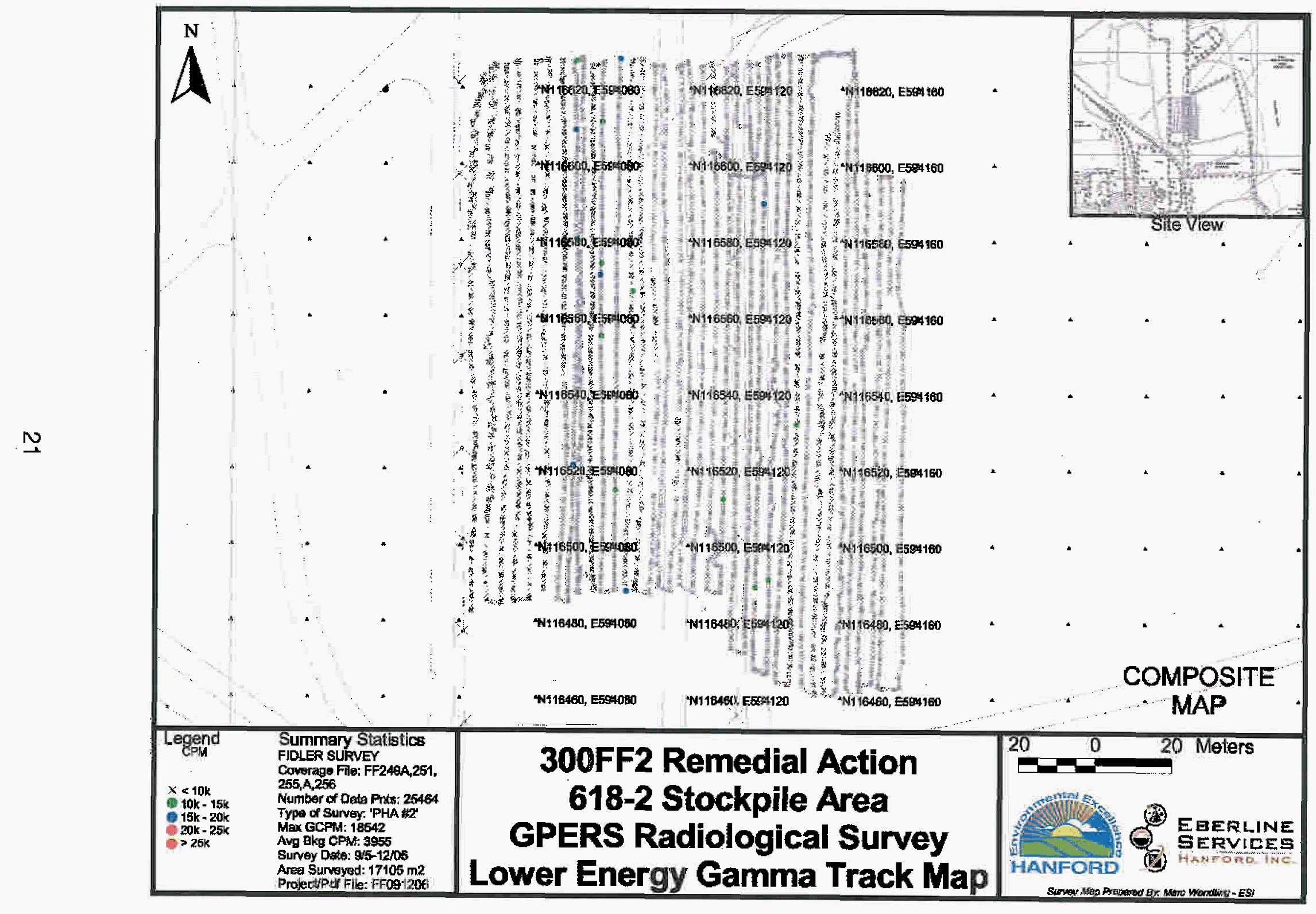

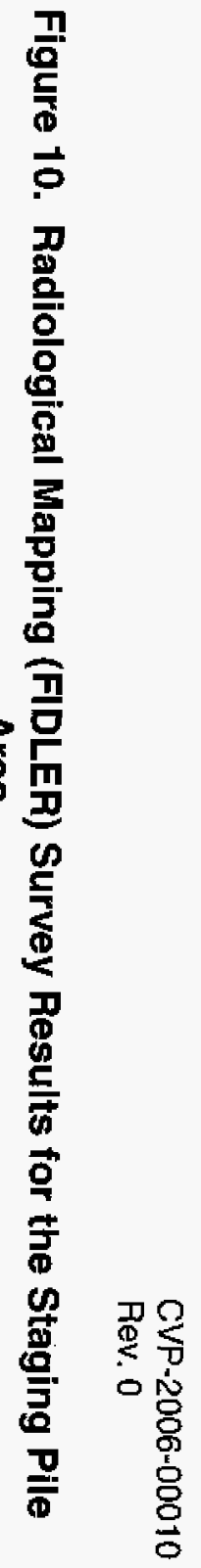




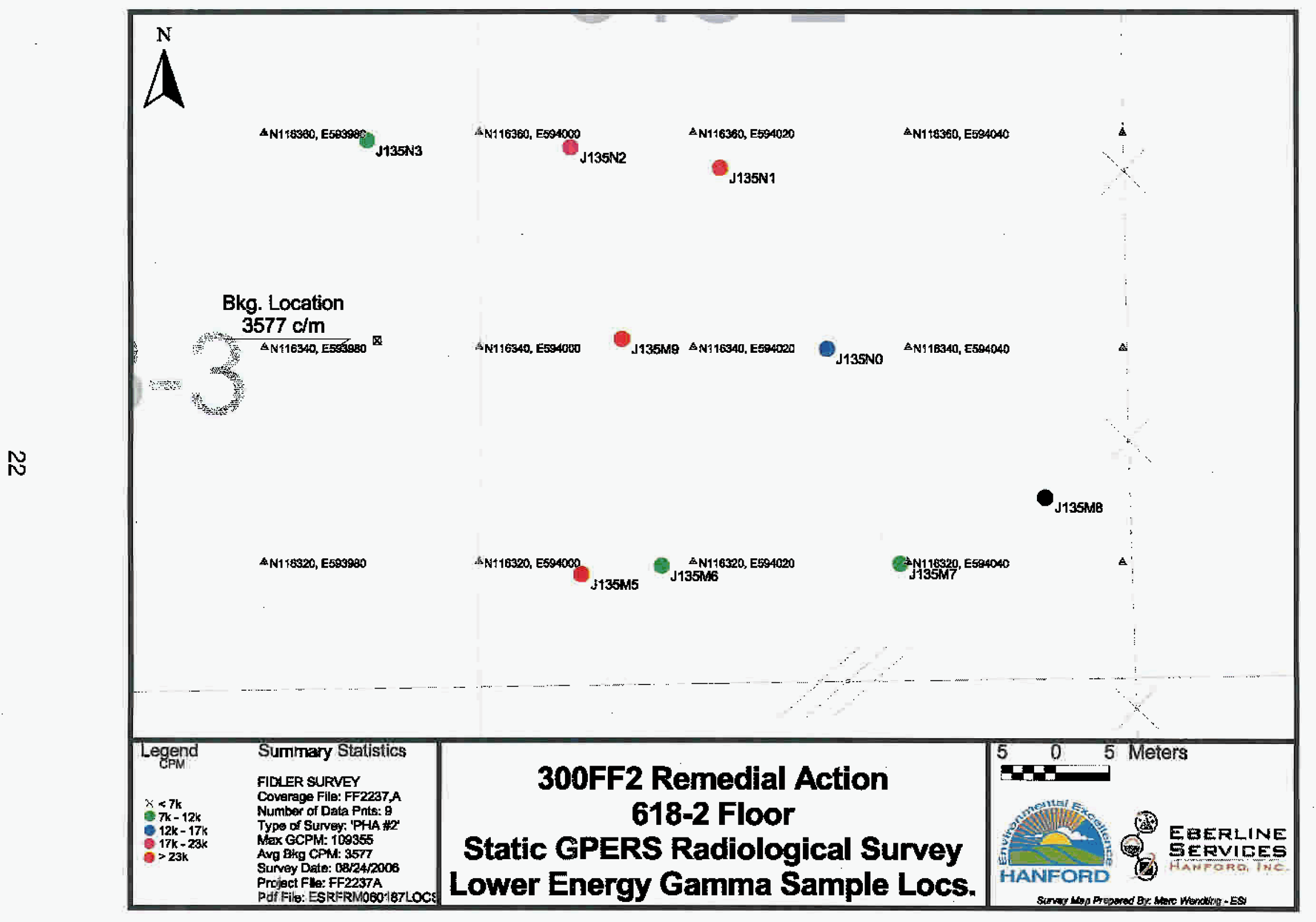




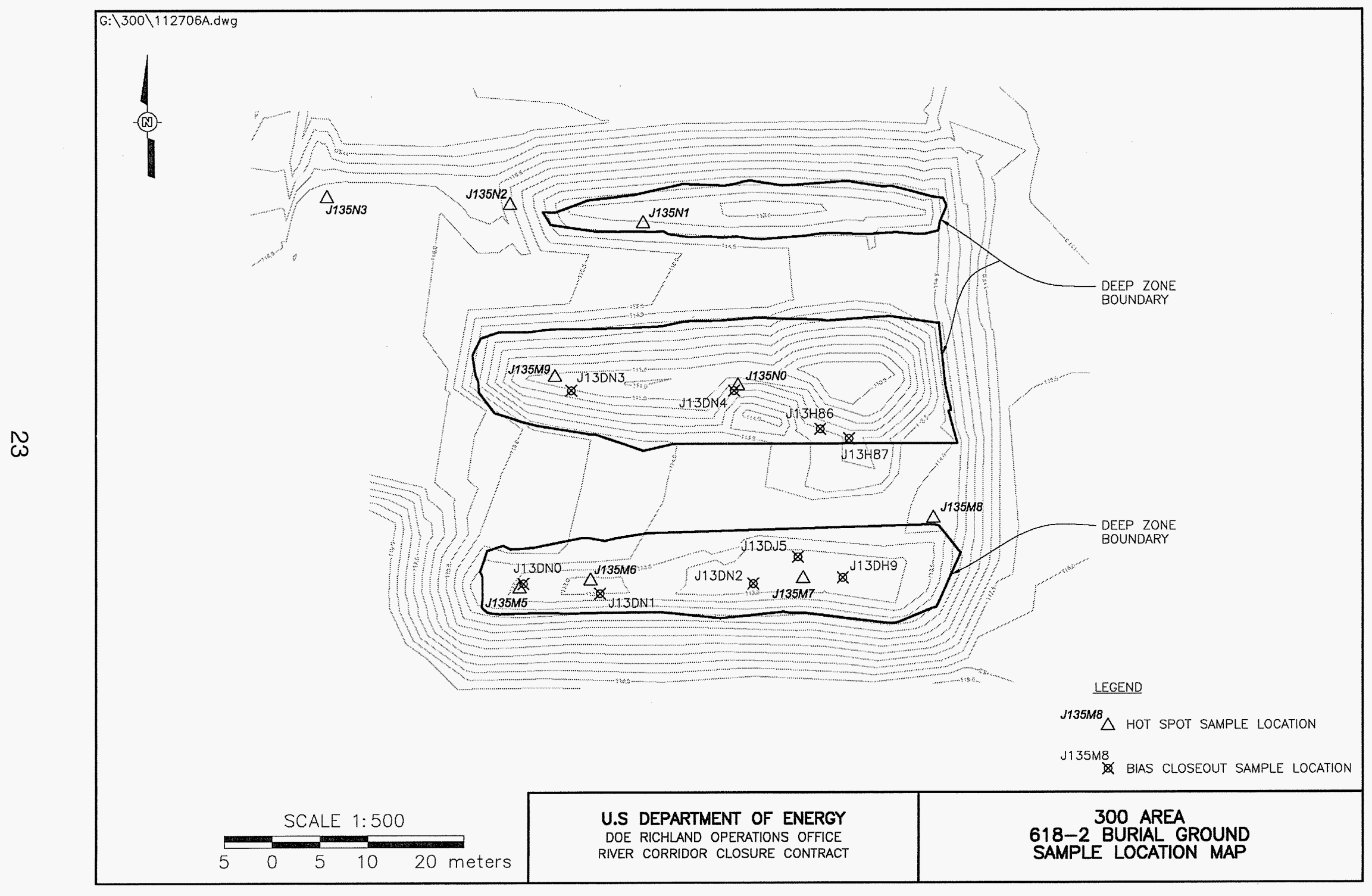

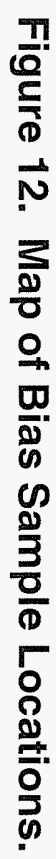




\subsection{CLEANUP VERIFICATION SAMPLING AND ANALYSIS}

Final cleanup verification samples were collected on September 6 and 7, 2006, to confirm acceptability of residual contaminant concentrations in the soil at the 618-2 waste site (WCH 2006a, 2006b). Each verification sample was a composite formed by combining soil collected at four randomly selected nodes within each sampling area. The sample design methodology and sample location figures are presented in the calculation brief for verification sample design in Appendix $D$. The final verification samples were submitted to offsite laboratories for analysis using approved EPA analytical methods as required per the SAP (DOE-RL 2004a).

The 618-2 Burial Ground verification sampling events involved four decision units including a shallow zone, deep zone, overburden soil stockpile, and staging pile/decontamination pad footprint. The staging pile/decontamination pad supported the 618-2, 618-3, and 618-8 Burial Ground excavations and, therefore, arsenic and silver were added as COPCs for staging pile/decontamination pad only. The division of the site excavation into shallow zone and deep zone decision units, as shown on the sample design figures in Appendix $\mathrm{D}$, is a function of the applicable RAGs. The direct exposure, groundwater protection, and river protection RAGs are applicable to soils within $4.6 \mathrm{~m}(15 \mathrm{ft})$ of the ground surface. This soil zone is referred to as the shallow zone. The groundwater protection and river protection RAGs are applicable to soils

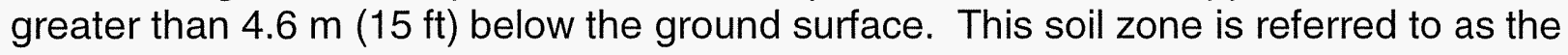
deep zone. The 618-2 site consisted of both a shallow and a deep zone decision unit. The site was excavated to a depth of approximately $6 \mathrm{~m}(19.7 \mathrm{ft})$, with the shallow zone consisting of the excavation sidewalls to a depth of $4.6 \mathrm{~m}(15 \mathrm{ft})$ and the deep zone consisting of the excavation sidewalls below $4.6 \mathrm{~m}(15 \mathrm{ft})$ together with the floor of the excavation. All deep zone samples were collected below $4.6 \mathrm{~m}(15 \mathrm{ft})$.

The number of decision subunits is determined by the overall footprint area of the decision unit, with the default number of verification samples for each decision unit being four composite samples. The required number of verification samples for each of the decision units associated with the 618-2 Burial Ground waste site are listed in Table 4.

Table 4. Number of Verification Samples for 618-2 Burial Ground.

\begin{tabular}{|c|c|c|c|c|}
\hline $\begin{array}{c}\text { Decision } \\
\text { Unit }\end{array}$ & $\begin{array}{c}\text { Waste Site } \\
\text { Footprint }\left(\mathrm{ft}^{2}\right)\end{array}$ & $\begin{array}{c}\text { Size Classification } \\
\left(\mathrm{ft}^{2}\right)\end{array}$ & $\begin{array}{c}\text { Decision } \\
\text { Subunits }\end{array}$ & $\begin{array}{c}\text { Verification } \\
\text { Samples }\end{array}$ \\
\hline Residual soil (Shallow Zone) & 32,570 & Small $(<100,000)$ & 1 & 4 composite \\
\hline Residual soil (Deep Zone) & 12,665 & Small $(<100,000)$ & 1 & 4 composite \\
\hline Residual soil (Overburden) & 20,270 & Small $(<100,000)$ & 1 & 4 composite \\
\hline $\begin{array}{c}\text { Residual soil } \\
\text { (Waste staging and } \\
\text { decontamination pad } \\
\text { footprint) }\end{array}$ & 250,131 & $\begin{array}{c}\text { Medium } \\
(>100,000 \text { to } 400,000)\end{array}$ & 4 & 16 composite \\
\hline
\end{tabular}


CVP-2006-00010

Rev. 0

\subsection{CLEANUP VERIFICATION DATA EVALUATION}

This section presents the evaluation and modeling of the 618-2 cleanup verification data for comparison with the data quality criteria and RAGs.

\subsection{DATA QUALITY ASSESSMENT PROCESS}

A data quality assessment (DQA) is performed to compare the verification sampling approach and resulting analytical data with the sampling and data quality requirements specified by the project objectives and performance specifications.

The DQA for the 618-2 waste site determined that the data are of the right type, quality, and quantity to support site verification decisions within specified error tolerances. All analytical data were found to be acceptable for decision-making purposes. The evaluation also verified that the sample design was sufficient to support clean site verification. The cleanup verification sample analytical data are stored in the Environmental Restoration database prior to archiving in the Hanford Environmental Information System and are summarized in Appendix B. The detailed DQA is presented in Appendix C.

\subsection{CONTAMINANTS OF CONCERN 95\% UPPER CONFIDENCE LIMIT}

The primary statistical calculation to support cleanup verification is the $95 \% \mathrm{UCL}$ on the arithmetic mean of the data. The $95 \% \mathrm{UCL}$ values for each COC are computed for the decision unit. Prior to calculating the $95 \%$ UCL, the individual sample results are reviewed and, as appropriate, adjusted per the SAP (DOE-RL 2004a). This process is summarized below.

Radionuclides: The laboratory-reported value is used in the calculation of the $95 \%$ UCL. In cases where the laboratory does not report a value for data qualified with a "U" (i.e., less than the detection limit), one-half of the minimum detectable activity is used in the calculation of the $95 \% \mathrm{UCL}$.

Nonradionuclides: For data flagged with a "U" (i.e., less than the detection limit), a value equal to one-half the practical quantitation limit is used for in the calculation of the 95\% UCL, as required by Washington Administrative Code (WAC) 173-340-740[7][g] (1996). If greater than half of the sample results for a given nonradionuclide COC are below detection, the statistical value is set equal to the maximum concentration detected (i.e., versus computing a $95 \% \mathrm{UCL}$ ).

Statistical calculations for 618-2 verification data are presented in the $95 \%$ UCL calculation briefs (Appendix D), with results shown in Table 5. Uranium background concentrations are accounted for in the shallow zone, deep zone, overburden soils and combined staging pile and decontamination pad area by subtracting the background 
concentration from the statistical value. Anthropogenic and naturally occurring radionuclide background are accounted for in the overburden soil only by subtracting the background concentration from the statistical value.

Table 5. Statistical Cleanup Verification Data Set. (2 Pages) ${ }^{a}$

\begin{tabular}{|c|c|c|c|c|c|}
\hline cocs & $\begin{array}{l}\text { Hanford Site } \\
\text { Background }\end{array}$ & Shallow Zone & Deep Zone & Overburden & $\begin{array}{c}\text { Staging Pile/ } \\
\text { Decon Pad }\end{array}$ \\
\hline \multicolumn{6}{|c|}{ Radionuclide Concentration (pCi/g) } \\
\hline Americium-241 & NA & 0.54 & 6.52 & $.023 \mathrm{U}$ & 0.15 \\
\hline Cesium-137 & $1.1^{b}$ & 1.5 & 0.914 & $0(<B G)$ & 0.032 \\
\hline Cobalt-60 & $0.008^{b}$ & $0.021 \mathrm{U}$ & $0.023 \mathrm{U}$ & $0.013 \mathrm{U}$ & $0.020 \mathrm{U}$ \\
\hline Europium-152 & NA & $0.055 \mathrm{U}$ & $0.068 \mathrm{U}$ & $0.054 \mathrm{U}$ & $0.052 \mathrm{U}$ \\
\hline Europium-154 & $0.033^{b}$ & $0.070 \mathrm{U}$ & $0.080 \mathrm{U}$ & $0.037 \mathrm{U}$ & $0.070 \mathrm{U}$ \\
\hline Europium-155 & $0.054^{b}$ & $0.069 \mathrm{U}$ & $0.13 \mathrm{U}$ & $0.0077 \mathrm{U}$ & $0.064 \mathrm{U}$ \\
\hline Nickel-63 & NA & $0.498 \mathrm{U}$ & $0.261 \mathrm{U}$ & $0.648 \mathrm{U}$ & $0.85 \mathrm{U}$ \\
\hline Tritium & NA & $0.0938 \mathrm{U}$ & $0.788 \mathrm{U}$ & $0.862 \mathrm{U}$ & 0.98 \\
\hline Plutonium-238 & $0.004^{b}$ & $0.056 \mathrm{U}$ & 1.08 & $0.038 \mathrm{U}$ & $0.067 \mathrm{U}$ \\
\hline Plutonium-239/240 & $0.025^{\mathrm{b}}$ & 5.2 & 72.9 & $0.065 \mathrm{U}$ & 1.8 \\
\hline Plutonium-241 & NA & $14.5 \mathrm{U}$ & 34.3 & $1.32 \mathrm{U}$ & $2.5 \mathrm{U}$ \\
\hline Strontium-90 & $0.18^{b}$ & $0.037 \mathrm{U}$ & 7.4 & $0(<B G) U$ & $0.055 \mathrm{U}$ \\
\hline Uranium-233/234 & $1.1^{\mathrm{b}}$ & 0.930 & 108 & $0(<B G)$ & 0.339 \\
\hline Uranium-235 & $0.11^{b}$ & 0.062 & 2.24 & $0(<B G) U$ & 0.0066 \\
\hline Uranium-238 & $1.1^{b}$ & 1.07 & 111 & $0(<B G)$ & 0.294 \\
\hline \multicolumn{6}{|c|}{ Nonradionuclide Concentration $(\mathrm{mg} / \mathrm{kg}$ ) } \\
\hline Arsenic & 6.5 & 1.9 & 1.4 & 2.6 & 3.4 \\
\hline Barium & 132 & 73.6 & 76.9 & 70.9 & 67.5 \\
\hline Cadmium & $0.81^{c}$ & $0.09 \mathrm{U}$ & $0.09 \mathrm{U}$ & $0.09 \mathrm{U}$ & $1.4 \mathrm{U}$ \\
\hline Chromium & 18.5 & 6.6 & 5.7 & 6.0 & 6.4 \\
\hline Lead & 10.2 & 4.9 & 5.5 & 4.2 & 4.0 \\
\hline Selenium & $0.78^{c}$ & 0.76 & 0.78 & 1.0 & $1.4 \mathrm{U}$ \\
\hline Silver & 0.73 & NA & NA & NA & $2.9 \mathrm{U}$ \\
\hline Tin & $N A^{d}$ & 2.3 & 2.9 & 2.4 & $28.8 \mathrm{U}$ \\
\hline
\end{tabular}


Table 5. Statistical Cleanup Verification Data Set. (2 Pages) ${ }^{a}$

\begin{tabular}{|c|c|c|c|c|c|}
\hline cocs & $\begin{array}{c}\text { Hanford Site } \\
\text { Background }\end{array}$ & Shallow Zone & Deep Zone & Overburden & $\begin{array}{c}\text { Staging Pile/ } \\
\text { Decon Pad }\end{array}$ \\
\hline Uranium (total) & 3.21 & 4.17 & 338 & 1.06 & 3.32 \\
\hline
\end{tabular}

${ }^{a}$ Laboratory data, including the minimum detectable activity or practical quantitation limit for the individual cleanup verification samples, are included in Appendix B and the 95\% UCL calculation brief in Appendix D.

b DOE-RL, 1996, Hanford Site Background: Part 2, Soil Background for Radionuclides, DOE/RL-96-12, Rev. 0, U.S. Department of Energy, Richland Operations Office, Richland, Washington.

c Hanford Site-specific background not available. Value is from Ecology publication 94-115 (Ecology 1994).

${ }^{d}$ No Hanford Site-specific background is available.

$\mathrm{BG}=$ background $\quad \mathrm{NA}=$ not applicable

$\mathrm{COC}=$ contaminant of concern $\quad \mathrm{U}=$ undetected

\subsection{BIASED SAMPLE RESULTS}

Nine biased grab samples were collected. The sample locations are shown in Figure 12. Five were located at suspect hot spots as determined from post-excavation radiological surveys. Bias samples were also collected below the location of the safe and also below the location of the drums. Two bias samples were collected in an area southwest of the test pit to verify the adequacy of removal of the plutonium soil contamination (Figures 5 and 12).

Statistical analysis (e.g., calculation of a $95 \%$ UCL value) is inappropriate for evaluation of biased samples; therefore, the sample results for each biased sample are evaluated using the maximum detected activity for each $\mathrm{COC}$ and comparing the value directly to the cleanup level. Table 6 provides a comparison of the maximum result of the nine biased samples against the cleanup criteria. Individual sample results are provided in Appendix B.

Table 6. Maximum Results from Biased Sampling. (2 Pages)

\begin{tabular}{|c|c|c|c|c|}
\hline \multirow{2}{*}{$\begin{array}{l}\text { Contaminant of } \\
\text { Concern }\end{array}$} & \multirow{2}{*}{ Maximum Result } & \multicolumn{2}{|c|}{$\begin{array}{l}\text { Industrial Land-Use Soil } \\
\text { Cleanup Level }\end{array}$} & \multirow{2}{*}{$\begin{array}{l}\text { Does the Maximum Result } \\
\text { Meet the Cleanup Level? }\end{array}$} \\
\hline & & $\begin{array}{c}\text { Direct } \\
\text { Exposure }\end{array}$ & $\begin{array}{l}\text { Groundwater } \\
\text { Protection }\end{array}$ & \\
\hline \multicolumn{5}{|c|}{ Nonradionuclides (mg/kg) } \\
\hline Arsenic & 3.3 & 58 & NA & Yes \\
\hline Barium & 109 & 4,900 & NA & Yes \\
\hline Cadmium & 0.20 & 139 & NA & Yes \\
\hline Chromium & 10.4 & $>1,000,000$ & NA & Yes \\
\hline Lead & 6.8 & 1,000 & NA & Yes \\
\hline Selenium & 1.2 & 17,500 & NA & Yes \\
\hline Silver & 0.6 & 17,500 & NA & Yes \\
\hline Tin & 42.5 & $>1,000,000$ & NA & Yes \\
\hline
\end{tabular}


CVP-2006-00010

Rev. 0

Table 6. Maximum Results from Biased Sampling. (2 Pages)

\begin{tabular}{|c|c|c|c|c|}
\hline \multirow{2}{*}{$\begin{array}{l}\text { Contaminant of } \\
\text { Concern }\end{array}$} & \multirow{2}{*}{ Maximum Result } & \multicolumn{2}{|c|}{$\begin{array}{l}\text { Industrial Land-Use Soil } \\
\text { Cleanup Level }\end{array}$} & \multirow{2}{*}{$\begin{array}{l}\text { Does the Maximum Result } \\
\text { Meet the Cleanup Level? }\end{array}$} \\
\hline & & $\begin{array}{c}\text { Direct } \\
\text { Exposure }\end{array}$ & $\begin{array}{l}\text { Groundwater } \\
\text { Protection }\end{array}$ & \\
\hline Total uranium $^{a}$ & 148 & 505 & 385 & Yes \\
\hline Aroclor-1254 & 0.0069 & 65.6 & 0.438 & Yes \\
\hline \multicolumn{5}{|c|}{ Radionuclides } \\
\hline Americium-241 & 9.19 & 210 & NA & Yes \\
\hline Cesium-137 & 1.12 & 25 & NA & Yes \\
\hline Plutonium-238 & 1.57 & 155 & NA & Yes \\
\hline Plutonium-239/240 & 91.6 & 245 & NA & Yes \\
\hline Plutonium-241 & 32.9 & 12,900 & NA & Yes \\
\hline Strontium-90 & 7.20 & 2,500 & NA & Yes \\
\hline Uranium-233/234, & 47.8 & 169 & 129 & Yes \\
\hline Uranium-235 & 2.42 & 17 & 13 & Yes \\
\hline Uranium-238 $8^{\mathrm{b}, \mathrm{c}}$ & 50.1 & 164 & 125 & Yes \\
\hline
\end{tabular}

a Reported total uranium value is based on summation of laboratory-reported isotopic uranium concentrations.

b The industrial scenario groundwater protection selected soil cleanup level for total uranium (267 pCi/g) was calculated using RESRAD, based on the generic site model, with a length parallel to groundwater of $100 \mathrm{~m}$ (328 ft) and distribution coefficient values of $8.9 \mathrm{~mL} / \mathrm{g}$ for the contaminated unsaturated zone and $0 \mathrm{~mL} / \mathrm{g}$ for the saturated zone (with no unsaturated, uncontaminated zone) (DOE-RL 2004b).

c Values determined using uranium equilibrium isotopic ratios and the industrial scenario selected soil cleanup levels for total uranium as provided in the Remedial Design Report/Remedial Action Work Plan for the 300 Area (DOE-RL 2004b).

RESRAD $=$ RESidual RADioactivity (dose model) $\quad N A=$ not applicable

\subsection{SITE-SPECIFIC CLEANUP VERIFICATION MODEL}

The statistical values summarized in Table 5 were evaluated and used to develop a site-specific cleanup verification model. The 618-2 site cleanup verification model comprises two depth intervals: (1) the shallow zone and overburden and (2) the deep zone ( $4.6 \mathrm{~m}[15 \mathrm{ft}$ ] below surrounding grade). Based on the conservative assumption that residual contaminant levels in the deep zone data set extend uniformly to groundwater (as discussed in the RDR/RAWP [DOE-RL 2004b]), residual soil activities of uranium 233/234, uranium-235, and uranium-238 are predicted to reach groundwater but at concentrations below the RAG for total uranium $(30 \mu \mathrm{g} / \mathrm{L}=21.2 \mathrm{pCi} / \mathrm{g})$.

\subsection{RESRAD MODELING}

The individual radionuclide cleanup verification statistical values (Table 5) were entered into the RESidual RADioactivity dose assessment model (RESRAD) computer code, Version 6.30 (ANL 2005), to estimate the dose rate and to estimate the impact on groundwater and the river from residual $\mathrm{COC}$ concentrations. The individual 
contributions from the shallow zone and the deep zone, and the combined staging pile and decontamination footprint were separately evaluated for direct exposure pathways and groundwater protection. No RESRAD modeling was performed for the overburden because all radionuclides were either undetected or below background.

The RESRAD modeling methodologies, results, input values, and the site-specific cleanup verification model are included in the RESRAD calculation brief (Appendix D). Specific results from the calculations are discussed in the RAG evaluation section (Section 5.0).

\subsection{EVALUATION OF REMEDIAL ACTION GOAL ATTAINMENT}

This section demonstrates that remedial actions at the 618-2 waste site have achieved the RAGs developed to support industrial land use at the 300 Area as established in the ESD (EPA 2004) and documented in the RDR/RAWP (DOE-RL 2004b). Since the combined staging pile and decontamination pad area was located in an uncontaminated area, the sample results are compared against the unrestricted land-use soil cleanup levels listed in Table 2. Section 5.4 documents application of the WAC 173-340 threepart test to the shallow zone, deep zone, overburden, and combined staging pile and decontamination pad area. This test is required for nonradionuclide COCs only and is based on the most restrictive RAG for each zone.

\subsection{DIRECT EXPOSURE SOIL REMEDIAL ACTION GOALS ATTAINED}

\subsubsection{Radionuclides}

5.1.1.1 Direct Comparison to RAGs. The results of the RESRAD dose rate estimates for the combined shallow and deep zone and for the combined staging pile and decontamination pad area, all-pathways scenarios are presented in Figures 13 and 14, respectively. The dose rates represent the dose contributions from soils at relevant time periods. The dose rate is largest at present (year 2006), $1.19 \mathrm{mrem} / \mathrm{yr}$, for the combined shallow and deep zones. The maximum dose rate for the combined staging pile and decontamination pad area is $0.212 \mathrm{mrem} / \mathrm{yr}$ at present (year 2006). The estimated dose rate in the year 2018 is $0.999 \mathrm{mrem} / \mathrm{yr}$ for shallow and deep zone and $0.202 \mathrm{mrem} / \mathrm{yr}$ for the combined staging pile and decontamination pad area. The 2018 date corresponds to the 30-year site cleanup schedule of the Hanford Federal Facility Agreement and Consent Order (Ecology et al. 1989). All dose rate estimates are less than the $15 \mathrm{mrem} / \mathrm{yr}$ RAG. The RESRAD computations are presented in detail in the referenced calculation briefs summarized in Appendix D. 
Figure 13. RESRAD Analysis - All-Radionuclides, All-Pathways Dose Rate Estimate - Combined Shallow and Deep Zone.

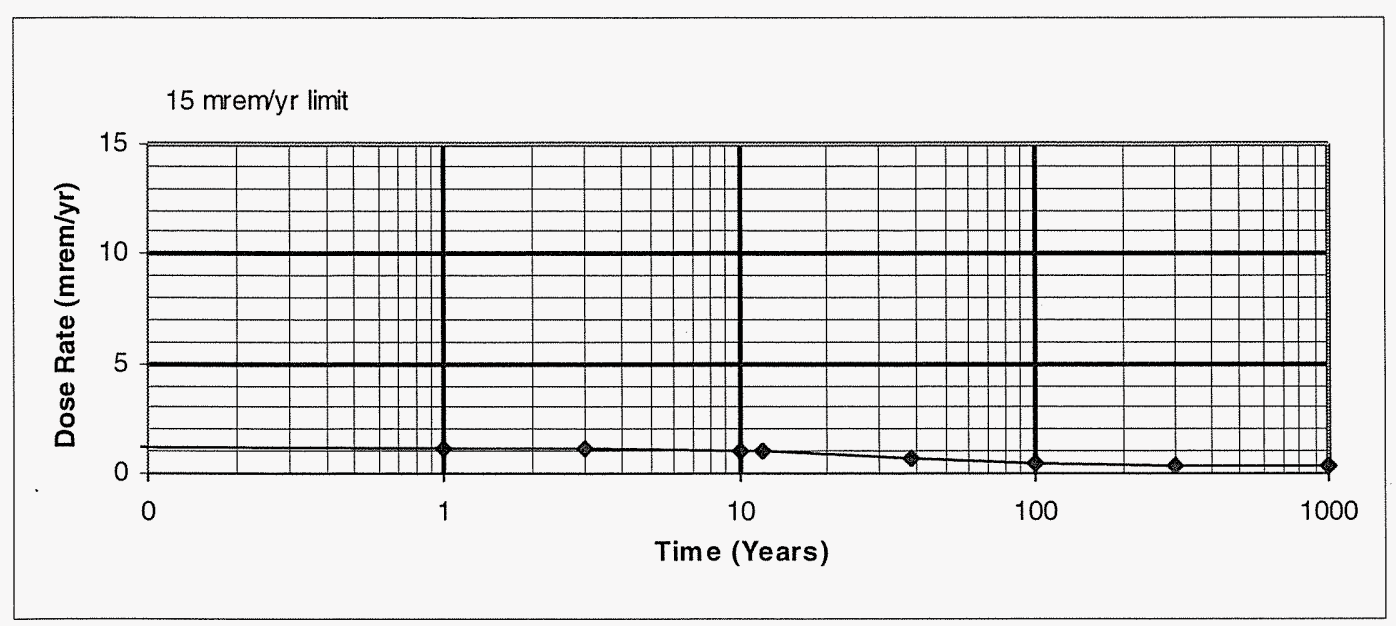

Figure 14. RESRAD Analysis - All-Radionuclides, All-Pathways Dose Rate Estimate - Combined Staging Pile and Decontamination Pad Areas.

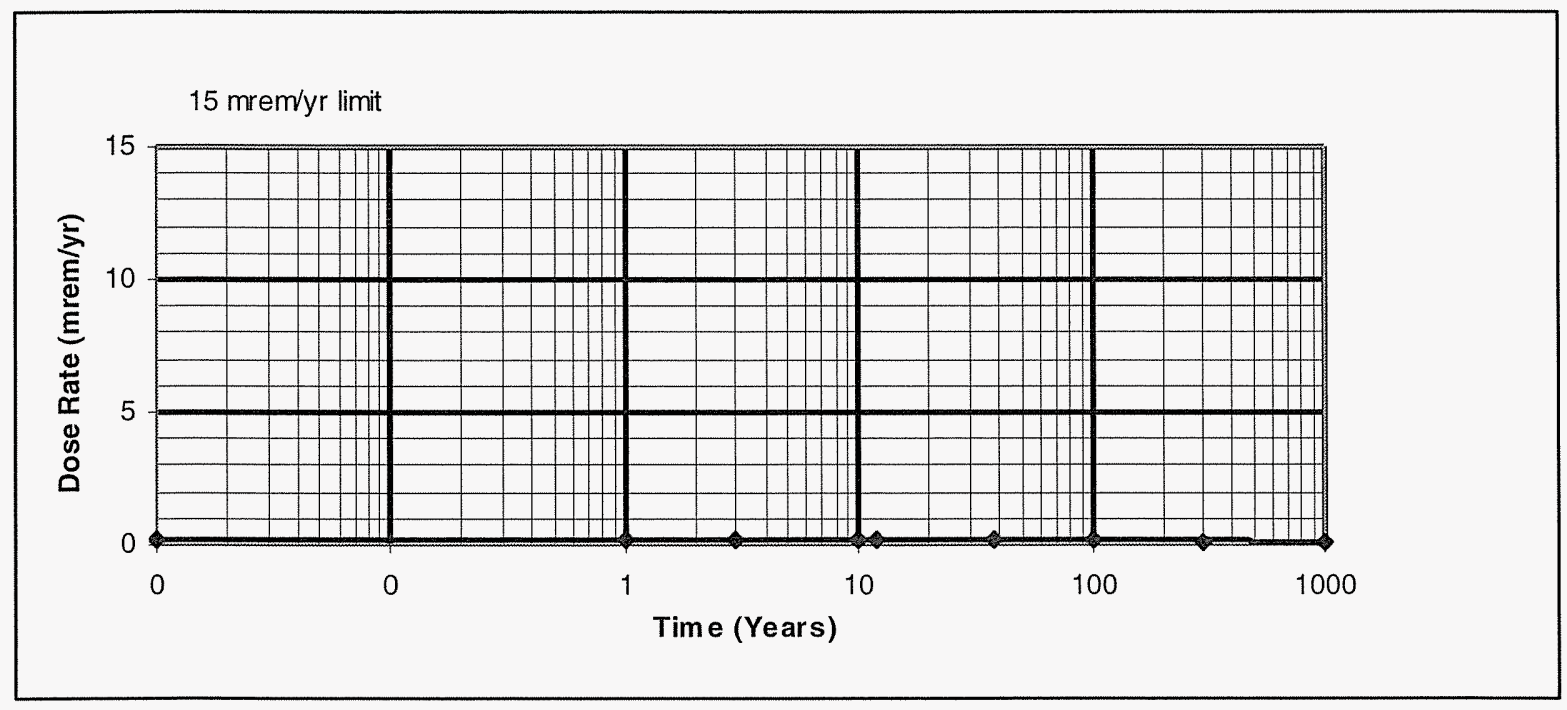

5.1.1.2 Radionuclide Risk. The results of the RESRAD analysis for radionuclide excess lifetime cancer risk (ELCR) for the combined shallow and deep zone, and the combined staging pile and decontamination area are shown in Figures 15 and 16, respectively. The maximum ELCR for the combined shallow and deep zone is $1.5 \times 10^{-5}$ and occurs at year 2006. The maximum ELCR for the combined staging pile and decontamination area is $9.58 \times 10^{-7}$ and occurs in year 2006. The ELCR predicted for the year 2018 is $1.19 \times 10^{-5}$ for the combined shallow and deep zone and $8.70 \times 10^{-7}$ for the combined staging pile and decontamination area. Therefore, both the combined shallow and deep zone ELCR and the combined staging pile and decontamination area attain the CERCLA risk range of $10^{-4}$ to $10^{-6}$. 


\subsubsection{Nonradionuclides}

5.1.2.1 Direct Comparison to RAGs. All nonradionuclide COCs were either not detected, detected below background levels, or below RAGs per the 300 Area RDR/RAWP as shown in Tables 5 and 6 . Therefore, all applicable RAGs are met.

5.1.2.2 Noncarcinogenic Hazard Quotient RAG Attained. For noncarcinogenic COCs, WAC 173-340-745(a) and (b) specify the evaluation of the hazard quotient which is given as a daily intake divided by a reference dose. For the shallow zone, tin and uranium were detected above background with calculated individual hazard quotients of $2.3 \times 10^{-6}$ and $8.3 \times 10^{-3}$, respectively, resulting in a cumulative hazard quotient of $8.3 \times 10^{-3}$. Selenium, tin, and uranium detected in the overburden resulted in individual respective hazard quotients of $5.7 \times 10^{-6}, 2.3 \times 10^{-6}$ and $2.1 \times 10^{-3}$ with a cumulative hazard quotient of $2.2 \times 10^{-3}$. For the combined staging pile and decontamination area, only uranium was detected above background resulting in both an individual and cumulative hazard quotient of $4.1 \times 10^{-2}$. This evaluation is shown in the $95 \% \mathrm{UCL}$ calculation brief (Appendix D). All calculated individual and cumulative hazard quotients were less than 1.0, therefore, the noncarcinogenic hazard quotient RAG has been attained for the 618-2 waste site.

Figure 15. RESRAD Analysis - Radionuclide Risk, All Pathways - Combined Shallow and Deep Zone.

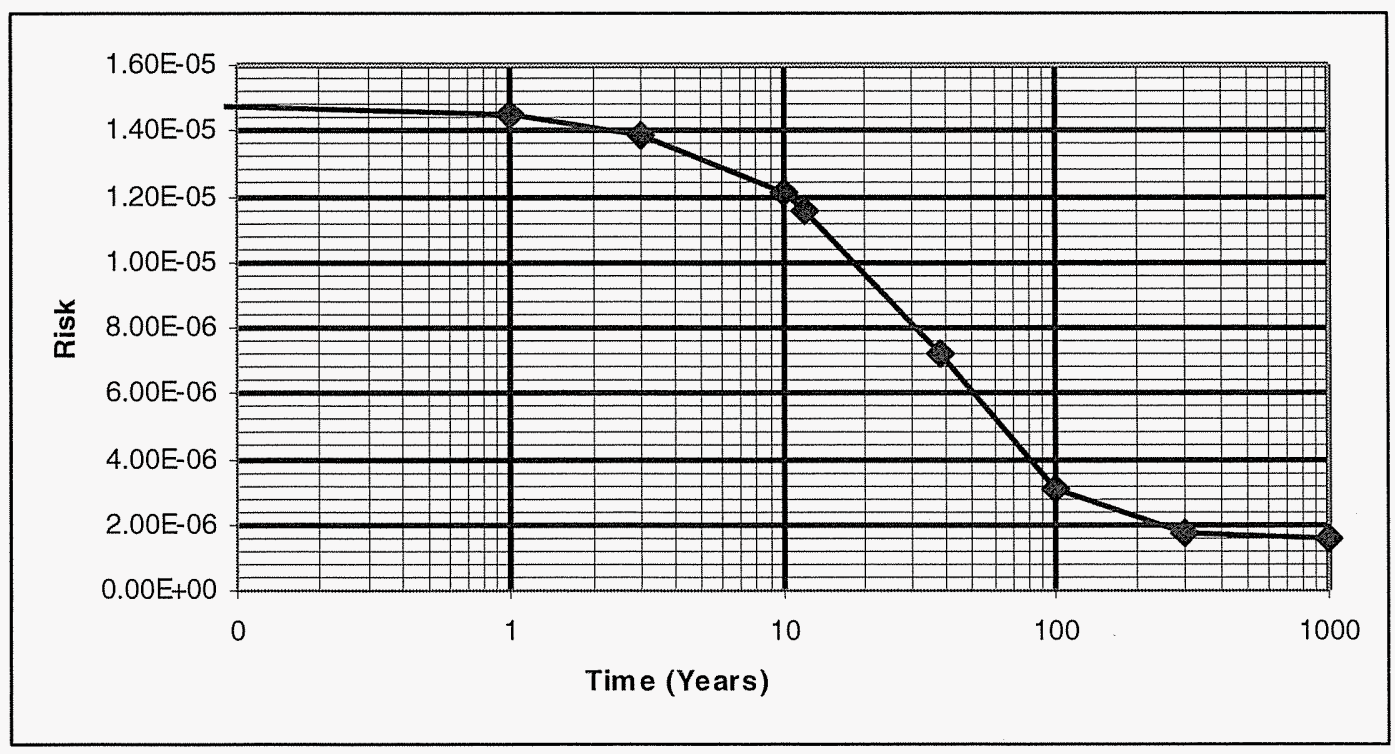


Figure 16. RESRAD Analysis - Radionuclide Risk - Combined Staging Pile and Decontamination Pad Areas.

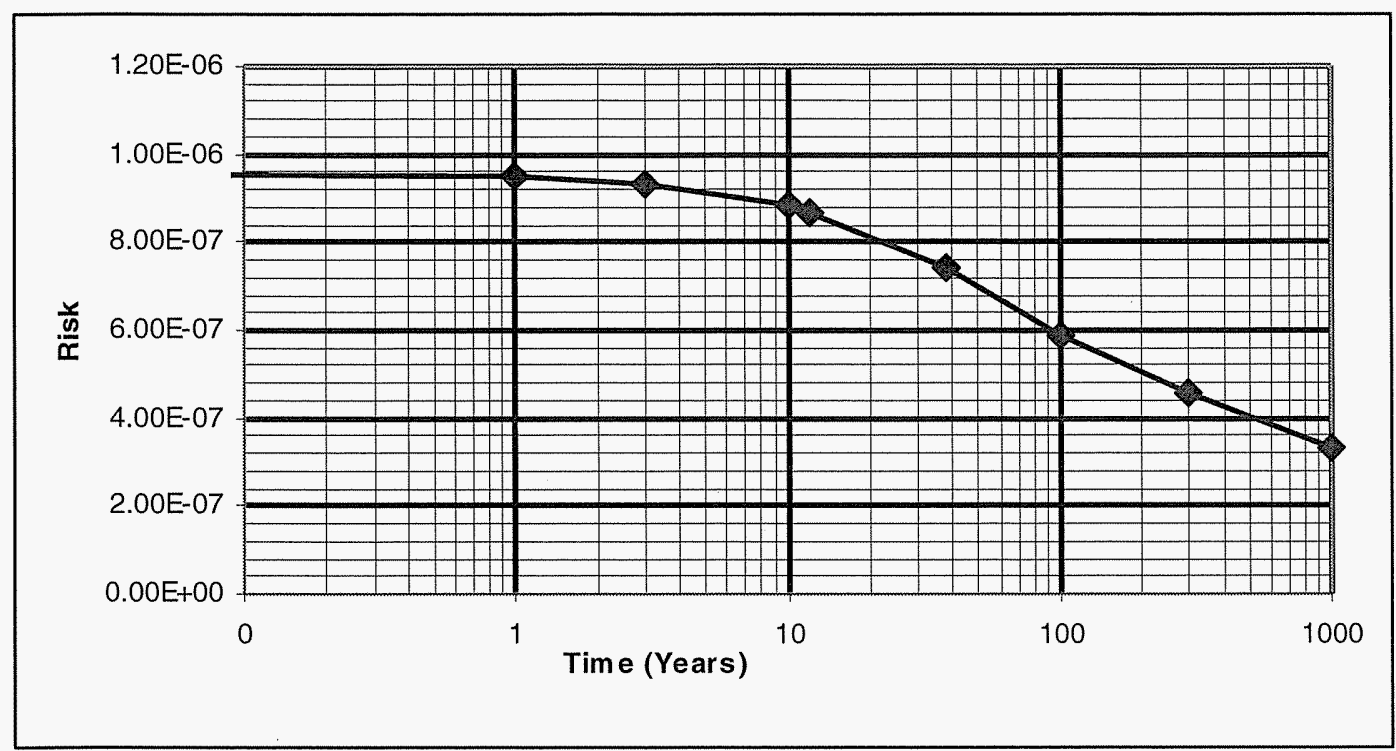

5.1.2.3 Carcinogenic Risk RAG Attained. For individual nonradionuclide carcinogenic COCs, the WAC 173-340-745(4)(a)(iii) Method C cleanup limits are based on an industrial land-use incremental cancer risk of $1 \times 10^{-5}$. The cumulative excess cancer risk for all nonradionuclide carcinogenic COCs must also be less than $1 \times 10^{-5}$ (WAC 173-340). Excess cancer risk was not calculated because the nonradionuclide carcinogenic COCs (arsenic and cadmium) were either not detected or detected below the statistical background levels.

\subsection{GROUNDWATER REMEDIAL ACTION GOALS ATTAINED}

\subsubsection{Radionuclides}

For the radionuclide contaminants of concern, only uranium-233/234, uranium-235 and uranium-238 are predicted to reach groundwater and at concentrations below the RAG established for the protection of groundwater (21.2 pCi/L) (EPA 2004, DOE-RL 2004b).

\subsubsection{Nonradionuclides}

All nonradionuclide COCs were either not detected, detected below background or below remedial action goals (as shown in Tables 5 and 6 ) and, therefore, are protective of the groundwater RAGs. 
CVP-2006-00010

Rev. 0

\subsection{COLUMBIA RIVER REMEDIAL ACTION GOALS ATTAINED}

\subsubsection{Radionuclides}

The cleanup verification statistical values for isotopic uranium were detected below the total uranium soil cleanup level of $267 \mathrm{pCi} / \mathrm{g}$, which has been determined to be protective of the Columbia River (EPA 2004, DOE-RL 2004b).

\subsubsection{Nonradionuclides}

All nonradionuclide COCs were either not detected, detected below background levels, or below remedial action goals (as shown in Tables 1 and 2) and, therefore, are protective of the Columbia River. Selenium was not detected in any of the verification samples collected from the combined waste staging pile/decontamination pad area; however, the laboratory practical quantification limit of $1.4 \mathrm{mg} / \mathrm{kg}$, exceeded the RAG of $1.0 \mathrm{mg} / \mathrm{kg}$ for protection of the Columbia River. Even if selenium were present at a concentration exceeding $1.4 \mathrm{mg} / \mathrm{kg}$, it has a large $\mathrm{K}_{\mathrm{d}}(150 \mathrm{~mL} / \mathrm{g})$ and therefore RESRAD predicts it will not reach groundwater or the Columbia River within 1,000 years.

\subsection{WAC 173-340 THREE-PART TEST FOR NONRADIONUCLIDES}

The WAC 173-340-740(7)(e) three-part test is applicable to nonradionuclide COCs and consists of the following criteria: (1) the cleanup verification statistical value must be less than the cleanup level, (2) no single detection can exceed two times the cleanup criteria, and (3) the percentage of samples exceeding the cleanup criteria must be less than $10 \%$. The most restrictive RAG (defined as the lowest of the direct exposure, groundwater protection, and river protection RAGs) is used for the test.

Selenium, tin, and uranium were the only nonradionuclides detected above background in the statistical verification data sets. The three-part test was not performed for selenium for the shallow zone, deep zone, and combined waste staging pile/ decontamination pad area because it was either not detected or was found at a concentration only slightly above the most restrictive RAG of $1.0 \mathrm{mg} / \mathrm{kg}$ for protection of the Columbia River and well below the direct exposure industrial land use RAG of $17,500 \mathrm{mg} / \mathrm{kg}$. Additionally, as stated in Section 5.3.2, even if selenium were present at a concentration exceeding $1.4 \mathrm{mg} / \mathrm{kg}$, it has a large $\mathrm{K}_{\mathrm{d}}(150 \mathrm{~mL} / \mathrm{g})$ and RESRAD predicts it will not reach groundwater within 1,000 years. The three-part test was not performed for tin because it was either not detected or was found at concentrations that RESRAD modeling predicts will not reach the groundwater within 1,000 years per the RDR/RAWP (DOE-RL 2004b). Table 7 summarizes the results of the WAC 173-340740[7][e] three-part test for the 618-2 cleanup verification nonradionuclide data sets for selenium and uranium in comparison to the most restrictive applicable RAG using industrial land use for the shallow zone, deep zone and overburden and unrestricted land use for the combined waste staging pile/decontamination pad area. The table lists the most restrictive RAG (from Table 1 and Table 2 as applicable), the maximum detected value, the total number of samples collected, and the percentage of samples 
exceeding the RAG. The final column of the table describes the result of applying the three criteria using the values listed in the preceding columns.

Table 7. Application of the WAC 173-340 Three-Part Test.

\begin{tabular}{|c|c|c|c|c|c|c|}
\hline $\begin{array}{l}\text { Contaminant } \\
\text { of Concern }\end{array}$ & $\begin{array}{c}\text { Most } \\
\text { Restrictive } \\
\text { Applicable } \\
\text { RAG }^{\mathrm{a}} \\
\text { (mg/kg) }\end{array}$ & $\begin{array}{c}\text { Statistical } \\
\text { Cleanup } \\
\text { Verification } \\
\text { Value } \\
(\mathrm{mg} / \mathrm{kg})^{\mathrm{b}}\end{array}$ & $\begin{array}{l}\text { Maximum } \\
\text { Detected } \\
\text { Cleanup } \\
\text { Verification } \\
\text { Value } \\
(\mathrm{mg} / \mathrm{kg})^{\mathrm{c}}\end{array}$ & $\begin{array}{c}\text { Total } \\
\text { Number } \\
\text { of } \\
\text { Samples }^{d}\end{array}$ & $\begin{array}{l}\text { Percentage of } \\
\text { Cleanup } \\
\text { Verification Data } \\
\text { Set Exceeding } \\
\text { RAG }^{\mathrm{e}}\end{array}$ & $\begin{array}{l}\text { Cleanup } \\
\text { Criteria } \\
\text { Attained? }\end{array}$ \\
\hline \multicolumn{7}{|c|}{ Shallow Zone } \\
\hline Uranium & 385 & 4.17 & 4.53 & 4 & 0 & Yes \\
\hline \multicolumn{7}{|c|}{ Deep Zone } \\
\hline Uranium & 385 & 338 & 501 & 4 & $25 \%$ & Yes $^{f}$ \\
\hline \multicolumn{7}{|c|}{ Overburden } \\
\hline Selenium & 1 & 1.0 & 1.0 & 4 & 0 & Yes \\
\hline \multicolumn{7}{|c|}{ Staging Pile/Decontamination Area } \\
\hline Uranium & 53 & 3.32 & 11.2 & 16 & 0 & Yes \\
\hline
\end{tabular}

a From Table 1, the most restrictive RAG is the soil RAG for protection of the Columbia River.

$b$ Criterion is statistical value cannot exceed most restrictive applicable RAG.

c Criterion is no single detection can exceed two times the most restrictive applicable RAG.

d Total number of samples in the decision unit includes field duplicate samples, which are included in the evaluation as separate samples.

e Criterion is percentage of data set exceeding the most restrictive applicable RAG cannot exceed $10 \%$.

${ }^{f}$ RESRAD modeling of the isotopes that comprise total uranium predict that the total concentration of uranium that may reach groundwater will not exceed the total uranium standard of $21.2 \mathrm{pCi} / \mathrm{L}$.

RAG = remedial action goal

WAC $=$ Washington Administrative Code

RESRAD = RESidual RADioactivity (dose model)

\subsection{STATEMENT OF PROTECTIVENESS}

This cleanup verification package demonstrates that remedial action at the 618-2 waste site has achieved the RAOs and corresponding RAGs established for the industrial land-use scenario in the ROD (EPA 2001), the ESD (EPA 2004), and the RDR/RAWP (DOE-RL 2004b). The contaminated materials from the site have been excavated and disposed of at ERDF. The remaining soil at the 618-2 site has been sampled, analyzed, and evaluated. Results indicate that the site supports future land uses that can be represented (or bounded) by the industrial land-use scenario and poses no threat to groundwater or the Columbia River. Institutional controls are required to prevent drilling or excavation into the deep zone. The 618-2 waste site is verified to be remediated in accordance with the ROD (EPA 2001) and the ESD (EPA 2004) and may be backfilled.

This cleanup verification package also demonstrates that the combined waste staging pile and decontamination pad area for the 618-2, 618-3, and 618-8 Burial Grounds do not preclude any future uses, as bounded by the rural-residential scenario, and provided 
for in the ESD (EPA 2004) and the RDR/RAWP (DOE-RL 2004b) and allow unrestricted use of shallow zone soils within this area. In consideration of this and because the waste staging pile area has no deep zone, no institutional controls are required at the waste staging pile location.

\subsection{REFERENCES}

40 CFR 141, "National Primary Drinking Water Regulations," Code of Federal Regulations, as amended.

65 FR 76708, "National Primary Drinking Water Regulations; Radionuclides; Final Rule," Federal Register, Vol. 65, No. 236, p. 76708, December 7, 2000.

ANL, 2005, RESRAD for Windows, Version 6.30, Argonne National Laboratory, Environmental Assessment Division, Argonne, Illinois.

BHI, 2001, Calculation of Total Uranium Activity Corresponding to a Maximum Contaminant Level for Total Uranium of 30 Micrograms per Liter in Groundwater, 0100X-CA-V0038, Rev. 0, Bechtel Hanford, Inc., Richland, Washington.

BHI, 2002, Protection of 300 Area Groundwater from Uranium-Contaminated Soils at Remediated Sites, BHI-01667, Rev. 0, Bechtel Hanford, Inc. Richland, Washington.

BHI, 2003, 300 Area Unrestricted Land-Use Radioactive Look-up Values RESRAD Calculation, Calculation Number 0300X-CA-V0042, Rev. 0, Bechtel Hanford Inc., Richland, WA.

BHI, 2004, 100 Area Radionuclide Lookup Values for the 1995 Interim Remedial Action Record of Decision, Calculation Number, 0100X-CA-V0046, Rev. 0, Bechtel Hanford, Inc., Richland, WA.

Comprehensive Environmental Response, Compensation, and Liability Act of 1980, 42 U.S.C. 9601 , et seq.

DOE Order 5400.5, Radiation Protection of the Public and the Environment, U.S. Department of Energy, Washington, D.C., as amended.

DOE-RL, 1996, Hanford Site Background: Part 2, Soil Background for Radionuclides, DOE/RL-96-12, Rev. 0, U.S. Department of Energy, Richland Operations Office, Richland, Washington. 
DOE-RL, 1998, Tri-Party Agreement Handbook Management Procedures, RL-TPA-90-0001, Guideline Number TPA-MP-14, "Maintenance of the Waste Information Data System (WIDS)," U.S. Department of Energy, Richland Operations Office, Richland, Washington.

DOE-RL, 2000, Focused Feasibility Study for the 300-FF-2 Operable Unit, DOE/RL-99-040, Rev. 0, U.S. Department of Energy, Richland Operations Office, Richland, Washington.

DOE-RL, 2004a, 300 Area Remedial Action Sampling and Analysis Plan, DOE/RL-2001-48, Rev. 1, U.S. Department of Energy, Richland Operations Office, Richland, Washington.

DOE-RL, 2004b, Remedial Design Report/Remedial Action Work Plan for the 300 Area, DOE/RL-2001-47, Rev. 1, U.S. Department of Energy, Richland Operations Office, Richland, Washington.

DOE-RL, 2005, Remedial Design Report/Remedial Action Work Plan for the 100 Area, DOE/RL-96-017, Rev. 5, U.S. Department of Energy, Richland Operations Office, Richland, Washington.

Ecology, 1994, National Background Soil Metals Concentrations in Washington State, Publication 94-115, Washington State Department of Ecology, Olympia, Washington.

Ecology, 2005, Cleanup Levels and Risk Calculations (CLARC) Database, Washington State Department of Ecology, Olympia, Washington, $<$ https://fortress.wa.gov/ecy/clarc/CLARCHome.aspx>.

Ecology, EPA, and DOE, 1989, Hanford Federal Facility Agreement and Consent Order, 2 vols., as amended, Washington State Department of Ecology,

U.S. Environmental Protection Agency, and U.S. Department of Energy, Olympia, Washington.

EPA, 1994, Guidance Manual for the Integrated Exposure Uptake Biokinetic Model for Lead in Children, EPA/540/R-93/081, Publication No. 9285.7-15-1, U.S. Environmental Protection Agency, Washington, D.C.

EPA, 2001, Interim Action Record of Decision for the 300-FF-2 Operable Unit, Hanford Site, Benton County, Washington, U.S. Environmental Protection Agency, Region 10, Seattle, Washington.

EPA, 2004, Explanation of Significant Differences for the 300-FF-2 Operable Unit Interim Record of Decision, U.S. Environmental Protection Agency, Region 10, Seattle, Washington. 
GE, 1954, Radiological Sciences Department Investigation Radiation Incident, Class 1, No. 343, General Electric, Richland, Washington.

WAC 173-340, 1996, "Model Toxics Control Act - Cleanup" Washington Administrative Code.

WCH, 2006a, Closeout Plan for the 618-2 Burial Ground, CCN 129577, Washington Closure Hanford, Richland, Washington.

WCH, 2006b, Remedial Sampling, Logbook EL-1395-12, Washington Closure Hanford, Richland, Washington. 
CVP-2006-00010

Rev. 0 
CVP-2006-00010

Rev. 0

\section{APPENDIX A}

\section{PHOTOGRAPHS}


CVP-2006-00010

Rev. 0

A-ii 

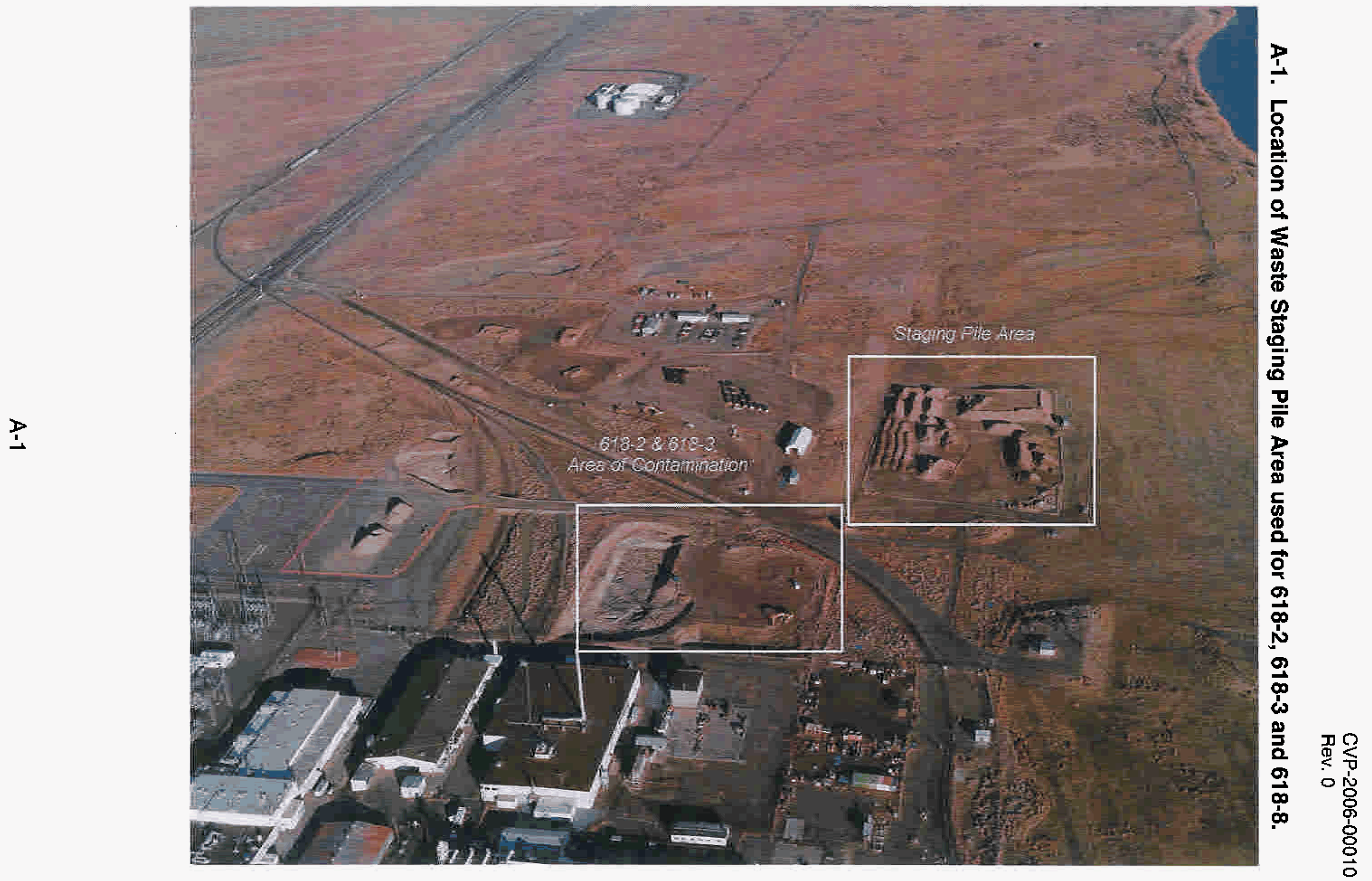
A-2. Historical Photo Showing 618-2 Burial Ground with Open Middle Trench.

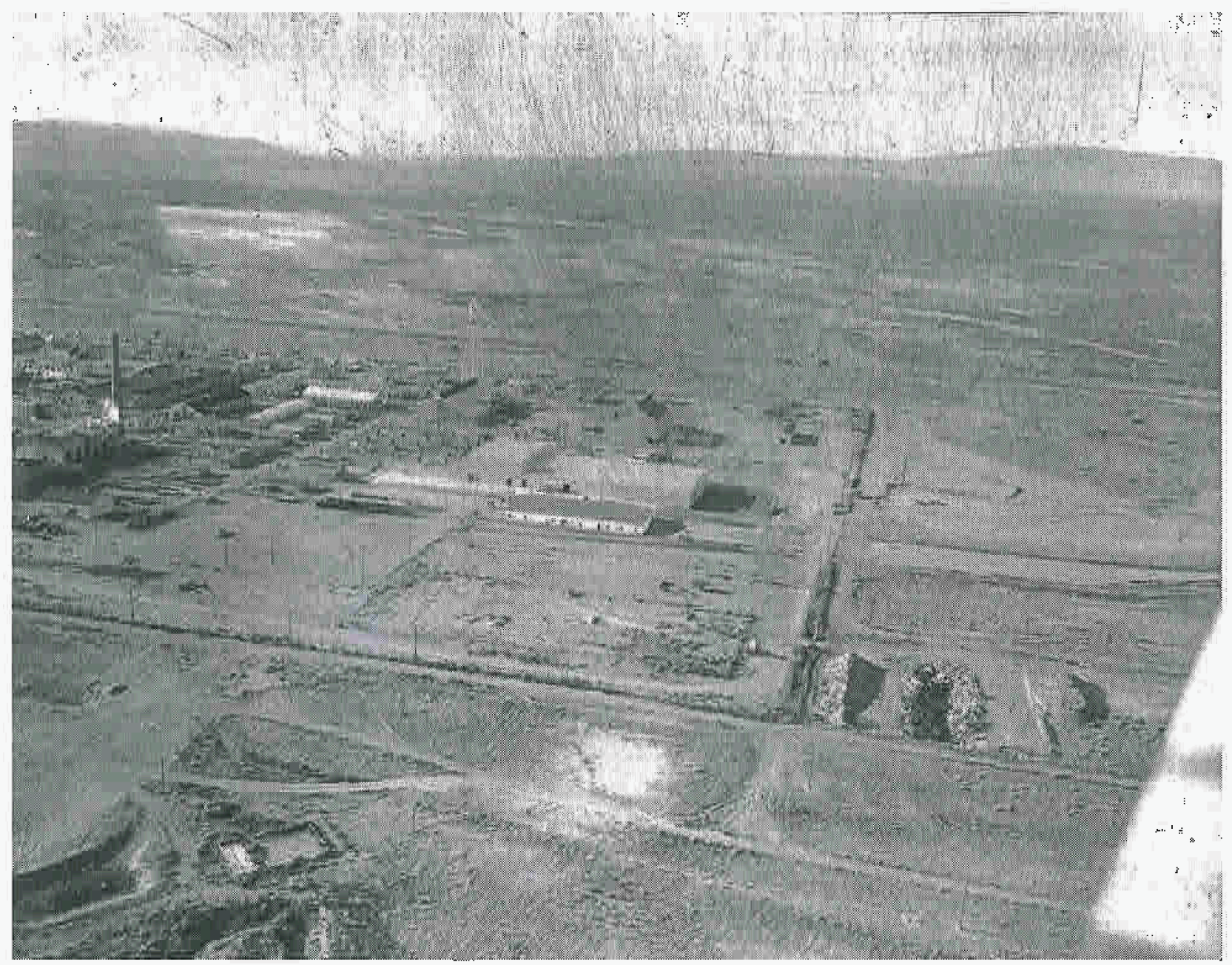


CVP-2006-00010

Rev. 0

A-3. Safe and Inner Containers Removed from 618-2 Burial Ground.

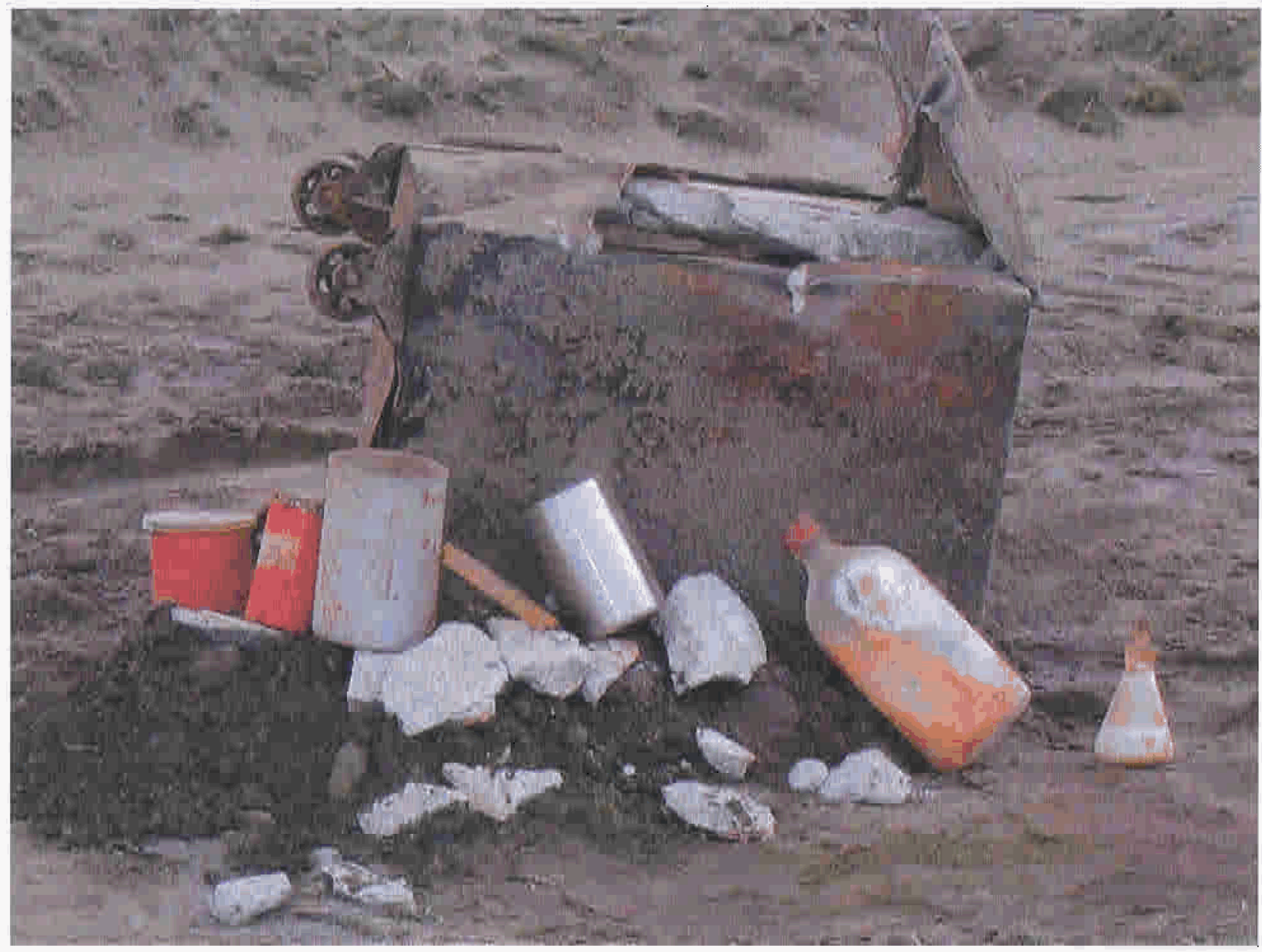


CVP-2006-00010

Rev. 0

\section{A-4. Laboratory Containers Found in 618-2 Burial Ground.}
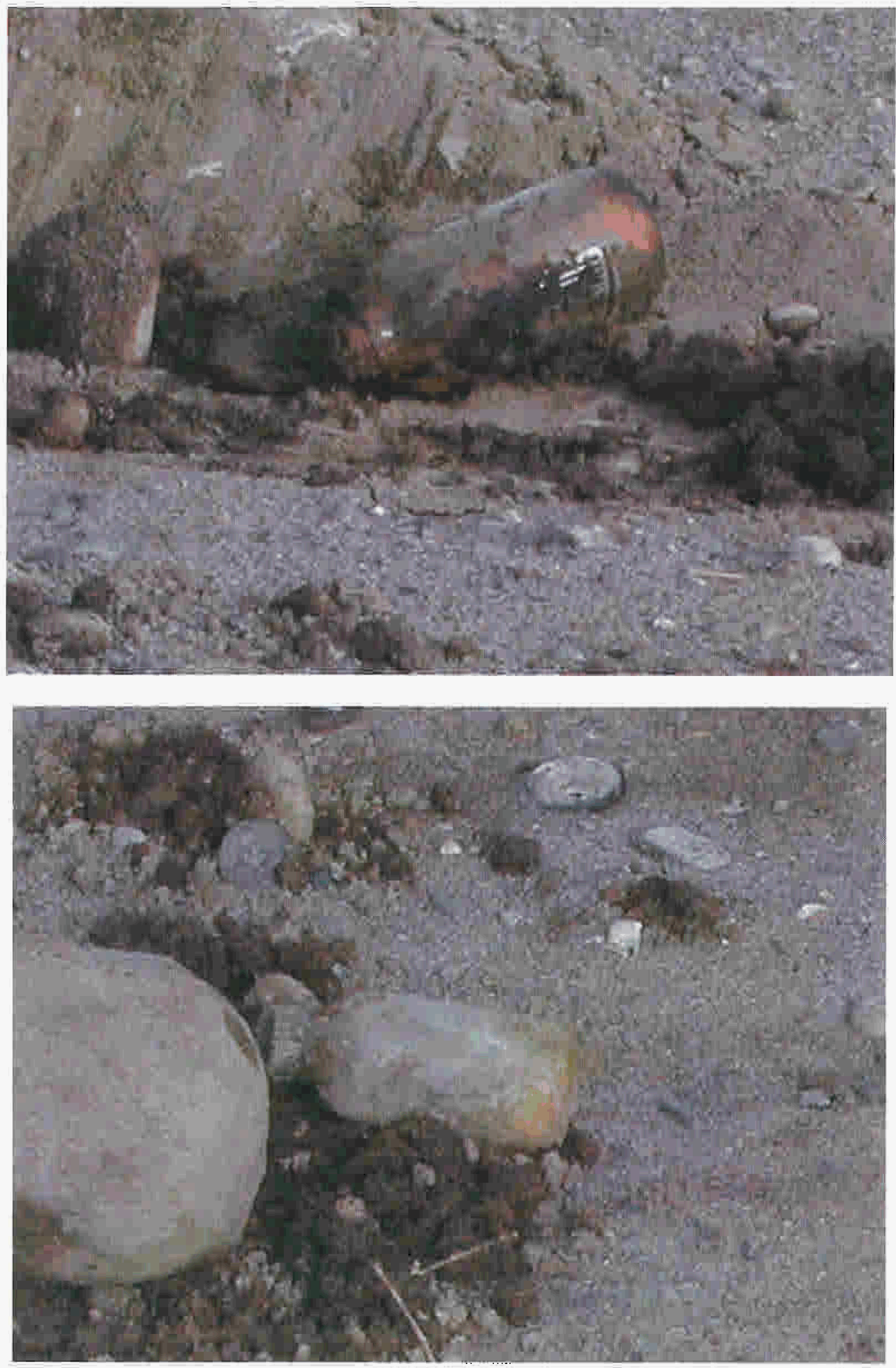
CVP-2006-00010

Rev. 0

A-5. Drums Removed from 618-2 Burial Ground and Overpacked.
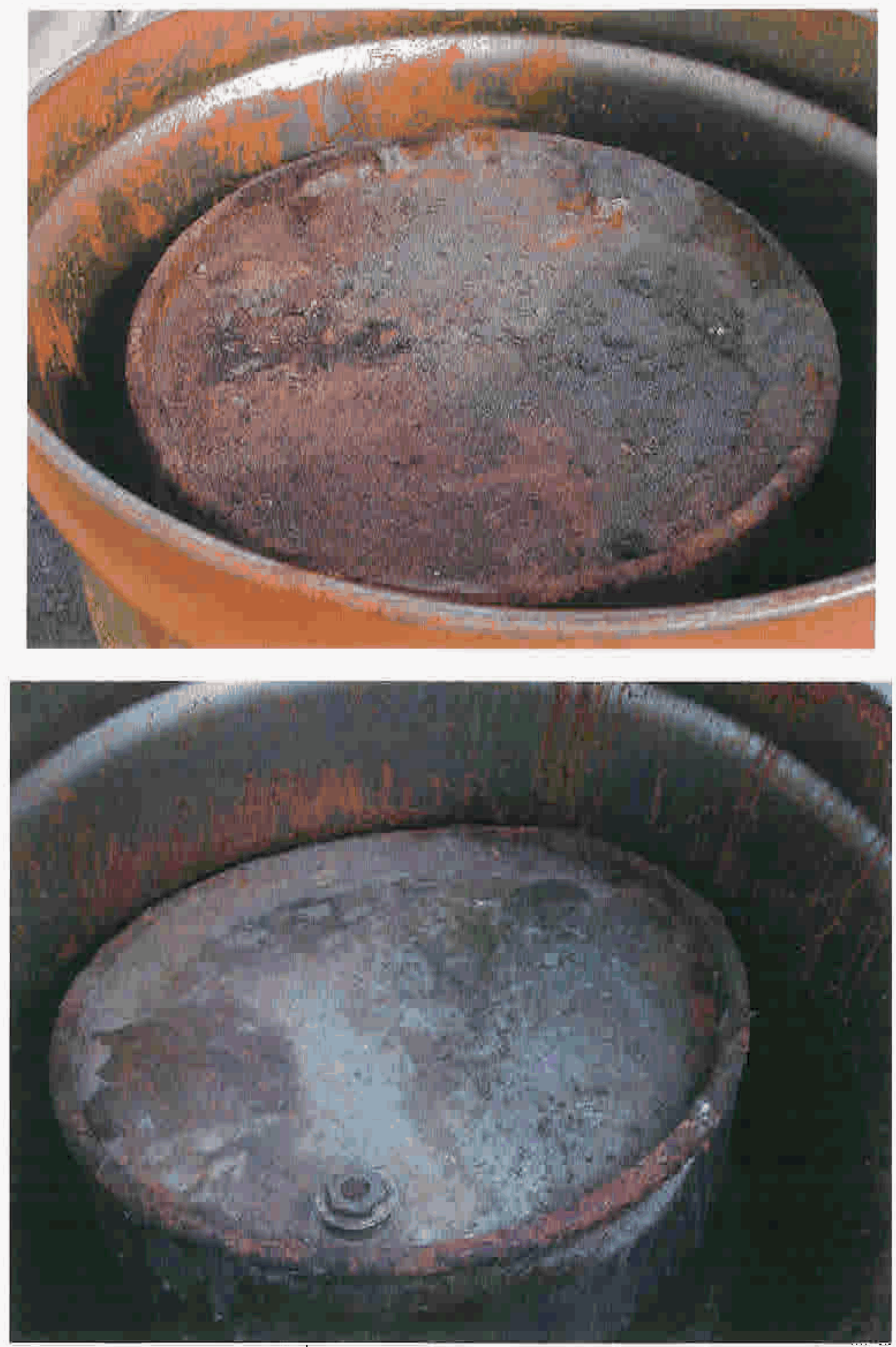
CVP-2006-00010

Rev. 0

A-6. "Hot Pig" Container Removed from 618-2 Burial Ground and Staged Until Characterization, Designation and Waste Shipment and Disposal Completed.

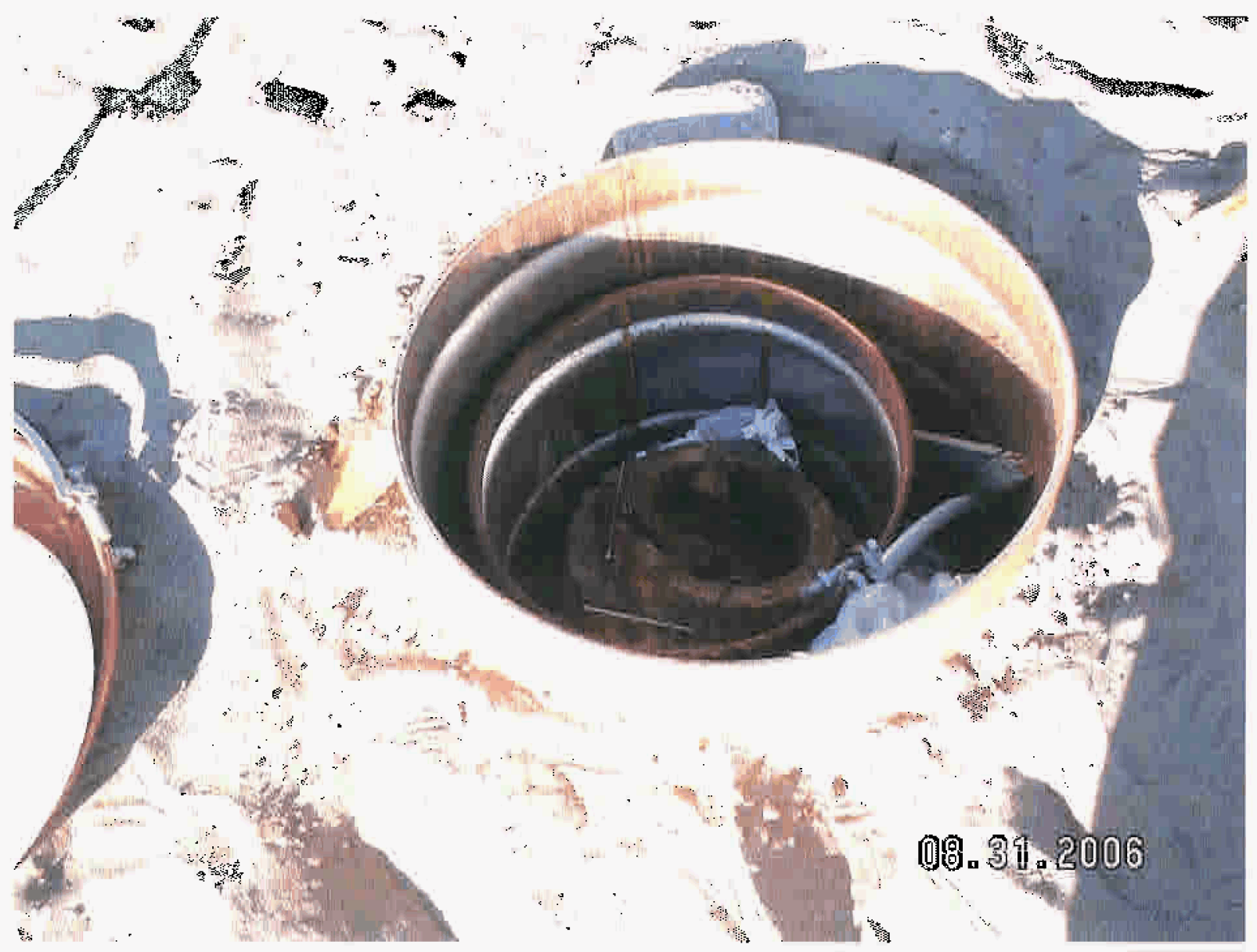


CVP-2006-00010

Rev. 0

\section{A-7. Photo of Test Pit Excavation Performed in Southeast Section of the Middle} Trench at 618-2 Burial Ground.

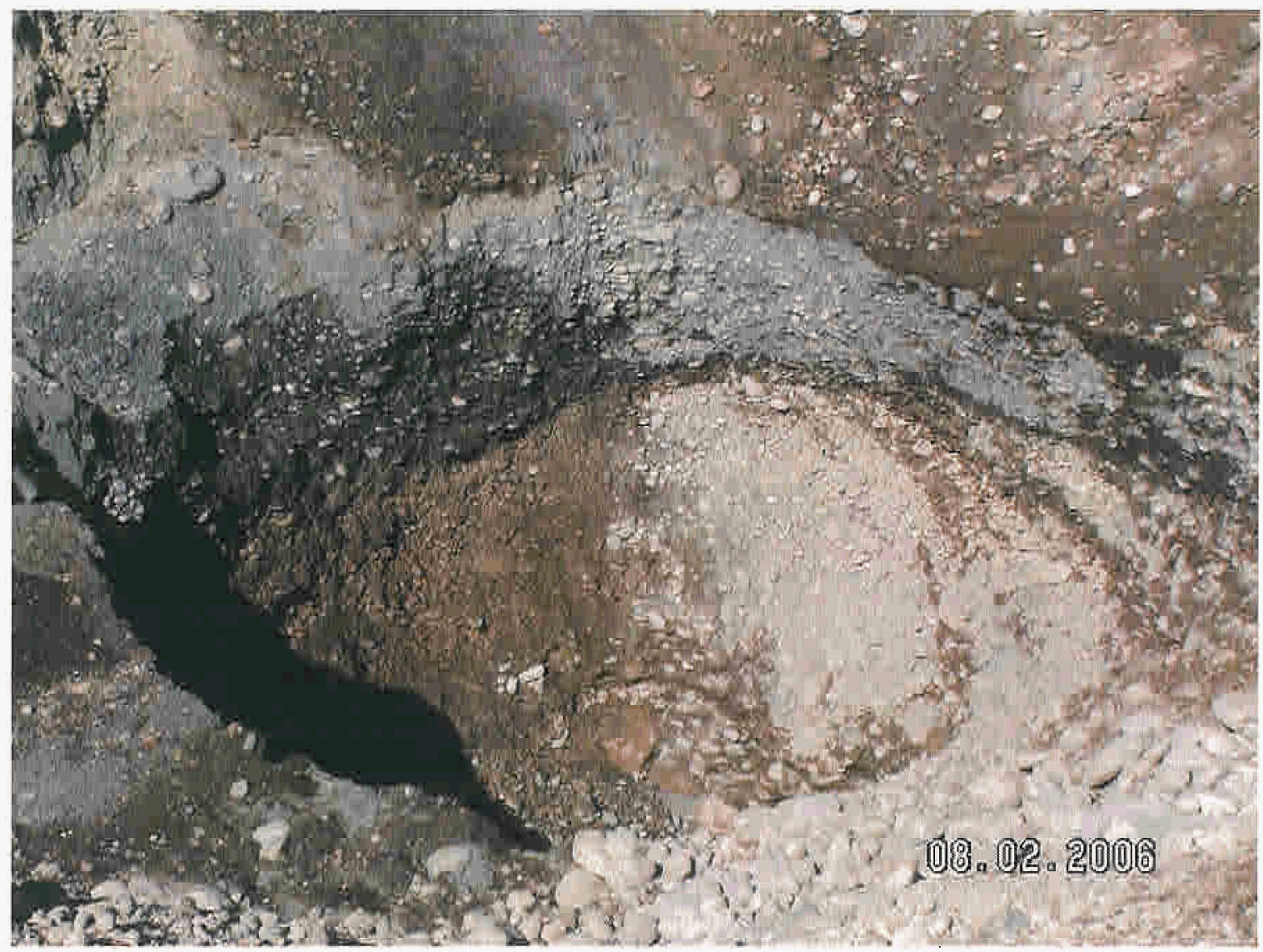


CVP-2006-00010

Rev. 0

A-8 
CVP-2006-00010

Rev. 0

APPENDIX B

SUMMARY OF ANALYTICAL SAMPLING RESULTS

B-i 
CVP-2006-00010

Rev. 0

B-ii 


\section{List of Analytical Tables}

Table B-1. 618-2 Shallow Zone Cleanup Verification Data. (2 Pages)

Table B-2. 618-2 Deep Zone Cleanup Verification Data. (2 Pages)

Table B-3. 618-2 Overburden Cleanup Verification Data. (2 Pages)

Table B-4. 618-2, 618-3, 618-8 Staging Pile/Decontamination Pad Cleanup Verification Data. (5 Pages)

Table B-5. 618-2 Verification Sample Results (Biased Samples). (3 Pages)

Table B-6. Test Pit Sample Results. (4 Pages)

Table B-7. Pothole Sample Results. (2 Pages)

Table B-8. FIDLER “Hot Spot” Sample Results. 
CVP-2006-00010

Rev. 0

B-iv 
Table B-1. 618-2 Shallow Zone Cleanup Verification Data. (2 Pages)

\begin{tabular}{|c|c|c|c|c|c|c|c|c|c|c|c|c|c|c|}
\hline \multirow{2}{*}{$\begin{array}{c}\text { Sampling } \\
\text { Area }\end{array}$} & \multirow{2}{*}{$\begin{array}{l}\text { Sample } \\
\text { Number }\end{array}$} & \multirow{2}{*}{$\begin{array}{c}\text { Sample } \\
\text { Date }\end{array}$} & \multicolumn{3}{|c|}{ Arsenic } & \multicolumn{3}{|c|}{ Barium } & \multicolumn{3}{|c|}{ Cadmium } & \multicolumn{3}{|c|}{ Chromium } \\
\hline & & & $\mathrm{mg} / \mathrm{kg}$ & $\mathbf{Q}$ & $\overline{P Q L}$ & $\mathrm{mg} / \mathrm{kg}$ & $\mathbf{Q}$ & $\overline{P Q L}$ & $\mathrm{mg} / \mathrm{kg}$ & $\mathbf{Q}$ & PQL & $\mathrm{mg} / \mathrm{kg}$ & $\mathbf{Q}$ & PQL \\
\hline $\mathrm{A} 1$ & J13DJ6 & $9 / 7 / 06$ & $1.8 \mathrm{E}+00$ & & $1.1 \mathrm{E}+00$ & $6.73 E+01$ & & $6 \mathrm{E}-02$ & $9 \mathrm{E}-02$ & $U$ & $9 \mathrm{E}-02$ & $7.1 \mathrm{E}+00$ & & $2.0 \mathrm{E}-01$ \\
\hline A3 & J13DJ9 & $9 / 7 / 06$ & $1.8 \mathrm{E}+00$ & & $1.1 \mathrm{E}+00$ & $7.13 \mathrm{E}+01$ & & $6 \mathrm{E}-02$ & $9 \mathrm{E}-02$ & $\bar{U}$ & $9 \mathrm{E}-02$ & $6.1 \mathrm{E}+00$ & & $2.0 \mathrm{E}-01$ \\
\hline A4 & J13DK0 & $9 / 7 / 06$ & $1.9 \mathrm{E}+00$ & & $1.1 \mathrm{E}+00$ & $5.85 E+01$ & & $6 \mathrm{E}-02$ & $9 \mathrm{E}-02$ & $U$ & $9 \mathrm{E}-02$ & $4.2 E+00$ & & $2.0 \mathrm{E}-01$ \\
\hline $\mathrm{A} 2$ & $\mathrm{~J} 13 \mathrm{DJ} 7$ & $9 / 7 / 06$ & $1.3 E+00$ & & $1.1 \mathrm{E}+00$ & $7.91 \mathrm{E}+01$ & & $6 \mathrm{E}-02$ & $9 \mathrm{E}+00$ & $\mathrm{U}$ & $9 \mathrm{E}+00$ & $4.5 E+00$ & & $2.0 \mathrm{E}-01$ \\
\hline $\begin{array}{l}\text { Duplicate } \\
\text { of } \mathrm{J} 13 \mathrm{DJ} 7 \\
\end{array}$ & J13DJ8 & 9/7/06 & -00 & & -00 & $6.98 \mathrm{E}+01$ & & 6E-02 & $9 \mathrm{E}+00$ & U & $9 \mathrm{E}+00$ & $4.6 \mathrm{E}+00$ & & 2.0E-01 \\
\hline $\begin{array}{l}\text { Split of } \\
\text { J13DJ7 }\end{array}$ & J13DK2 & 9/7/06 & $1.8 E+00$ & & 2.8E-01 & $7.98 \mathrm{E}+01$ & & 5.0E-01 & $1.4 \mathrm{E}-01$ & U & $1.4 \mathrm{E}-01$ & $5.5 \mathrm{E}+00$ & & 3.6E-01 \\
\hline $\begin{array}{c}\text { Equipment } \\
\text { Blank }\end{array}$ & J13DK1 & 9/7/06 & $1.9 \mathrm{E}+00$ & & $1.1 E+00$ & $5.85 E+01$ & & 6E-02 & 9E-02 & $U$ & 9E-02 & $4.2 \mathrm{E}+00$ & & $2.0 E-01$ \\
\hline
\end{tabular}

\begin{tabular}{|c|c|c|c|c|c|c|c|c|c|c|c|c|c|c|}
\hline \multirow{2}{*}{$\begin{array}{c}\text { Sampling } \\
\text { Area }\end{array}$} & \multirow{2}{*}{$\begin{array}{l}\text { Sample } \\
\text { Number }\end{array}$} & \multirow{2}{*}{$\begin{array}{c}\text { Sample } \\
\text { Date }\end{array}$} & \multicolumn{3}{|c|}{ Lead } & \multicolumn{3}{|c|}{ Selenium } & \multicolumn{3}{|c|}{ Tin } & \multicolumn{3}{|c|}{ Uranium } \\
\hline & & & $\mathrm{mg} / \mathrm{kg}$ & $\mathbf{Q}$ & $\overline{P Q L}$ & $\mathrm{mg} / \mathrm{kg}$ & $\mathbf{Q}$ & PQL & $\mathrm{mg} / \mathrm{kg}$ & $\mathbf{Q}$ & PQL & $\mathrm{mg} / \mathrm{kg}$ & $\mathbf{Q}$ & PQL \\
\hline A1 & J13DJ6 & $9 / 7 / 06$ & $5.6 \mathrm{E}+00$ & & $3.5 \mathrm{E}+00$ & $7.5 E-01$ & & $7.5 \mathrm{E}+00$ & $2.0 E+00$ & $J$ & $1.0 \mathrm{E}+00$ & $3.51 \mathrm{E}+00$ & & $7 E-03$ \\
\hline$\overline{\mathrm{A} 3}$ & J13DJ9 & $9 / 7 / 06$ & $3.7 \mathrm{E}+00$ & & $3.5 \mathrm{E}+00$ & $7.5 \mathrm{E}-01$ & $\bar{U}$ & $7.5 \mathrm{E}+00$ & $2.4 \mathrm{E}+00$ & $J$ & $1.0 \mathrm{E}+00$ & $3.35 \mathrm{E}+00$ & & 7E-03 \\
\hline $\mathrm{A} 4$ & J13DK0 & $9 / 7 / 06$ & $3.3 E+00$ & & $3.5 \mathrm{E}+00$ & 7.5E-01 & $U$ & $7.5 \mathrm{E}+00$ & $2.1 \mathrm{E}+00$ & $J$ & $1.0 E+00$ & $4.53 E+00$ & & $7 E-03$ \\
\hline $\mathrm{A} 2$ & J13DJ7 & $9 / 7 / 06$ & $3.4 \mathrm{E}+00$ & & $3.4 \mathrm{E}+00$ & 7.4E-01 & U & 7.4E-01 & $2.1 \mathrm{E}+00$ & $\mathrm{~J}$ & $1.0 \mathrm{E}+00$ & $3.72 E+00$ & & $7 E-03$ \\
\hline $\begin{array}{c}\text { Duplicate } \\
\text { of J13DJ7 }\end{array}$ & J13DJ8 & $9 / 7 / 06$ & +00 & & +00 & 7.4E-01 & U & 7.4E-01 & $2.2 \mathrm{E}+00$ & $J$ & $1.0 E+00$ & $3.35 E+00$ & & 7E-03 \\
\hline $\begin{array}{l}\text { Split of } \\
\text { J13DJ7 } \\
\end{array}$ & J13DK2 & 9/7/06 & $2.6 \mathrm{E}+00$ & & $1.5 \mathrm{E}-01$ & 5.5E-01 & & 1.7E-01 & $2.8 E+00$ & U & $2.8 E+00$ & +01 & & $2.08 \mathrm{E}-02$ \\
\hline $\begin{array}{c}\text { Equipment } \\
\text { Blank }\end{array}$ & J13DK1 & 9/7/06 & 7.0E-01 & & 1.2E-01 & $2.5 \mathrm{E}-01$ & $U$ & 2.5E-01 & $6.8 \mathrm{E}-01$ & $\mathrm{~J}$ & $3.4 \mathrm{E}-01$ & $5.88 \mathrm{E}-01$ & & 7E-03 \\
\hline
\end{tabular}

$J=$ estimated

MDA = minimum detectable activity

$\mathrm{NA}=$ not analyzed

$P Q L=$ practical quantification limit

$Q=$ Qualifier

$U=$ undetected 
Table B-1. 618-2 Shallow Zone Cleanup Verification Data. (2 pages)

\begin{tabular}{|c|c|c|c|c|c|c|c|c|c|c|c|c|c|c|c|c|}
\hline \multirow{2}{*}{$\begin{array}{l}\text { Sample } \\
\text { Number }\end{array}$} & \multirow{2}{*}{$\begin{array}{c}\text { Sample } \\
\text { Date }\end{array}$} & \multicolumn{3}{|c|}{ Americium-241 } & \multicolumn{3}{|c|}{ Cesium-137 } & \multicolumn{3}{|c|}{ Cobalt-60 } & \multicolumn{3}{|c|}{ Europium-152 } & \multicolumn{3}{|c|}{ Europium-154 } \\
\hline & & $\mathrm{pCi} / \mathrm{g}$ & $\mathbf{Q}$ & MDA & $\mathrm{pCi} / \mathrm{g}$ & $\mathbf{Q}$ & MDA & $\mathrm{pCi} / \mathrm{g}$ & $\mathbf{Q}$ & MDA & $\mathrm{pCi} / \mathrm{g}$ & $\mathbf{Q}$ & MDA & $\mathrm{pCi} / \mathrm{g}$ & $\mathbf{Q}$ & MDA \\
\hline J13DJ6 & $9 / 7 / 06$ & $-1.0 \mathrm{E}-01$ & $\mathrm{U}$ & $7.3 \mathrm{E}-02$ & $2.24 E+00$ & & $3.9 E-02$ & $2.7 \mathrm{E}-02$ & $\mathrm{U}$ & 2.7E-02 & $8.4 \mathrm{E}-02$ & $U$ & $8.4 \mathrm{E}-02$ & 9.7E-02 & $\mathrm{U}$ & $9.7 E-02$ \\
\hline J13DJ9 & $9 / 7 / 06$ & $-3.7 E-02$ & $\bar{U}$ & $9.3 \mathrm{E}-02$ & $4.8 E-02$ & $\mathrm{U}$ & $4.8 \mathrm{E}-02$ & $4.8 \mathrm{E}-02$ & $\mathrm{U}$ & $4.8 E-02$ & $1.3 \mathrm{E}-01$ & $\mathrm{U}$ & 1.3E-01 & 1.7E-01 & $U$ & 1.7E-01 \\
\hline J13DK0 & $9 / 7 / 06$ & $8.1 \mathrm{E}-01$ & & -02 & $E-02$ & $\mathrm{U}$ & $2.1 \mathrm{E}-02$ & 2.3E-02 & $\mathrm{U}$ & $2.3 \mathrm{E}-02$ & $4.7 E-02$ & $\mathrm{U}$ & 4.7E-02 & $7.8 \mathrm{E}-02$ & $\mathrm{U}$ & 7.8E-02 \\
\hline J13DJ7 & $9 / 7 / 06$ & $5.6 \mathrm{E}-02$ & $\mathrm{U}$ & E-02 & $3.4 \mathrm{E}-02$ & & $1.7 \mathrm{E}-02$ & -02 & $\mathrm{U}$ & $4.6 \mathrm{E}-02$ & $\mathrm{E}-02$ & $U$ & $\mathrm{E}-02$ & $5.3 \mathrm{E}-02$ & $\mathrm{U}$ & $5.3 \mathrm{E}-02$ \\
\hline J13DJ8 & $9 / 7 / 06$ & $9.9 \mathrm{E}-02$ & $U$ & 1.1E-01 & 4.0E-02 & & 1.9E-02 & $1.7 E-02$ & $U$ & 1.7E-02 & 4.3E-02 & $U$ & 4.3E-02 & 5.1E-02 & $\mathrm{U}$ & 5.1E-02 \\
\hline J13DK2 & $9 / 7 / 06$ & $3.37 E-01$ & & 2.52E-02 & $5.00 E-02$ & & 1.57E-02 & 8.05E-03 & $\mathrm{U}$ & $1.84 \mathrm{E}-02$ & $-8.08 E-03$ & $\mathrm{U}$ & $3.70 \mathrm{E}-02$ & $-1.63 E-02$ & $\mathrm{u}$ & $5.89 E-02$ \\
\hline
\end{tabular}

\begin{tabular}{|c|c|c|c|c|c|c|c|c|c|c|c|c|c|c|c|c|}
\hline $\begin{array}{l}\text { Sample } \\
\text { Number }\end{array}$ & $\begin{array}{c}\text { Sample } \\
\text { Date }\end{array}$ & \multicolumn{3}{|c|}{ Europium-155 } & \multicolumn{3}{|c|}{ Nickel-63 } & \multicolumn{3}{|c|}{ Tritium } & \multicolumn{3}{|c|}{ Plutonium-238 } & \multicolumn{3}{|c|}{ Plutonium-239/240 } \\
\hline J13DJ6 & $9 / 7 / 06$ & $6.7 \mathrm{E}-02$ & $\bar{U}$ & $6.7 \mathrm{E}-02$ & $-1.71 E+00$ & $U$ & $3.8 \mathrm{E}+00$ & $-4.29 \mathrm{E}-01$ & $U$ & $2.4 \mathrm{E}+00$ & $5.1 \mathrm{E}-02$ & U & $4.9 \mathrm{E}-01$ & $3.55 \mathrm{E}-01$ & $\mathrm{U}$ & $3.9 \mathrm{E}-01$ \\
\hline J13DJ9 & $9 / 7 / 06$ & $1.8 \mathrm{E}-01$ & $\bar{U}$ & $1.8 \mathrm{E}-01$ & $8.08 \mathrm{E}-01$ & $U$ & $3.7 \mathrm{E}+00$ & $-8.46 \mathrm{E}-01$ & $U$ & $2.5 \mathrm{E}+00$ & 0 & U & 5.3E-01 & $6.9 \mathrm{E}-02$ & $\mathrm{U}$ & $5.3 \mathrm{E}-01$ \\
\hline J13DK0 & $9 / 7 / 06$ & $4.9 \mathrm{E}-02$ & $\bar{U}$ & $4.9 \mathrm{E}-02$ & \begin{tabular}{|l|}
$-5.40 \mathrm{E}-01$ \\
\end{tabular} & $U$ & $3.7 \mathrm{E}+00$ & $-2.26 \mathrm{E}-01$ & $\mathrm{U}$ & $2.5 E+00$ & 0 & $\mathrm{U}$ & $4.5 \mathrm{E}-01$ & $7.67 E+00$ & & $4.5 \mathrm{E}-01$ \\
\hline J13DJ8 & $9 / 7 / 06$ & $6.1 \mathrm{E}-02$ & $u$ & $6.1 \mathrm{E}-02$ & $-1.01 E+00$ & $u$ & $3.9 E+00$ & 4.77E-01 & $u$ & $2.4 \mathrm{E}+00$ & $6.8 \mathrm{E}-02$ & $u$ & $2.6 E-01$ & 7.18E-01 & & $2.6 \mathrm{E}-01$ \\
\hline J13DK2 & $9 / 7 / 06$ & 3.61E-02 & $u$ & 4.06E-02 & $5.95 \mathrm{E}+00$ & $u$ & $6.18 E+00$ & $8.82 \mathrm{E}-03$ & $u$ & $3.89 \mathrm{E}-02$ & $1.57 \mathrm{E}-02$ & $u$ & $2.92 \mathrm{E}-02$ & $1.73 \mathrm{E}+00$ & & 2.92E-02 \\
\hline
\end{tabular}

\begin{tabular}{|c|c|c|c|c|c|c|c|c|c|c|c|c|c|c|c|c|}
\hline \multirow{2}{*}{$\begin{array}{l}\text { Sample } \\
\text { Number }\end{array}$} & \multirow{2}{*}{$\begin{array}{c}\text { Sample } \\
\text { Date }\end{array}$} & \multicolumn{3}{|c|}{ Plutonium-241 } & \multicolumn{3}{|c|}{ Strontium-90 } & \multicolumn{3}{|c|}{ Uranium-233/234 } & \multicolumn{3}{|c|}{ Uranium-235 } & \multicolumn{3}{|c|}{ Uranium-238 } \\
\hline & & $\mathrm{pCi} / \mathrm{g}$ & 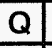 & MDA & $\mathrm{pCi} / \mathrm{g}$ & $\mathbf{Q}$ & MDA & $\mathrm{pCi} / \mathrm{g}$ & $Q$ & MDA & $\mathrm{pCi} / \mathrm{g}$ & $\mathbf{Q}$ & MDA & $\mathrm{pCi} / \mathrm{g}$ & $\mathbf{Q}$ & MDA \\
\hline J13DJ6 & $9 / 7 / 06$ & $1.32 \mathrm{E}+01$ & $\mathrm{U}$ & $1.9 \mathrm{E}+01$ & $6.3 \mathrm{E}-02$ & $U$ & $2.6 \mathrm{E}-01$ & $1.66 \mathrm{E}+00$ & & 1.7E-01 & $1.85 \mathrm{E}-01$ & $U$ & $2.0 \mathrm{E}-01$ & $1.90 \mathrm{E}+00$ & & $1.7 \mathrm{E}-01$ \\
\hline J13DJ9 & $9 / 7 / 06$ & $1.40 \mathrm{E}+01$ & $U$ & $2.2 E+01$ & $-6.5 \mathrm{E}-02$ & $U$ & $2.4 \mathrm{E}-01$ & $1.59 \mathrm{E}+00$ & & 2.1E-01 & $6.8 \mathrm{E}-02$ & $U$ & $2.6 \mathrm{E}-01$ & $2.20 \mathrm{E}+00$ & & $2.1 \mathrm{E}-01$ \\
\hline J13DK0 & $9 / 7 / 06$ & $4.27 \mathrm{E}+00$ & $U$ & $2.4 \mathrm{E}+01$ & $-7.0 \mathrm{E}-03$ & $\mathrm{U}$ & $2.2 \mathrm{E}-01$ & $2.20 \mathrm{E}+00$ & & $2.1 \mathrm{E}-01$ & $3.3 \mathrm{E}-02$ & $U$ & $2.5 \mathrm{E}-01$ & $2.14 \mathrm{E}+00$ & & $2.1 \mathrm{E}-01$ \\
\hline J13DJ7 & $9 / 7 / 06$ & $1.42 \mathrm{E}+01$ & $U$ & $2.0 \mathrm{E}+01$ & $-1.4 \mathrm{E}-02$ & $U$ & $2.2 \mathrm{E}-01$ & $1.71 \mathrm{E}+00$ & & 1.7E-01 & $2.14 \mathrm{E}-01$ & & $2.1 \mathrm{E}-01$ & $1.77 \mathrm{E}+00$ & & $1.7 \mathrm{E}-01$ \\
\hline J13DJ8 & $9 / 7 / 06$ & $9.65 E+00$ & $u$ & $1.8 \mathrm{E}+01$ & .02 & $\mathrm{u}$ & $2.5 \mathrm{E}-01$ & $1.83 \mathrm{E}+00$ & & 2.3E-01 & 1.11E-01 & U & $2.8 \mathrm{E}-01$ & $1.89 \mathrm{E}+00$ & & 2.3E-01 \\
\hline J13DK2 & 9/7/06 & $6.60 \mathrm{E}-01$ & $u$ & $9.79 \mathrm{E}-01$ & $9.71 \mathrm{E}-03$ & $\mathrm{U}$ & $1.26 \mathrm{E}-01$ & $5.02 \mathrm{E}+00$ & & 3.84E-02 & 1.15E-01 & & 3.23E-02 & $4.98 \mathrm{E}+00$ & & 2.61E-02 \\
\hline
\end{tabular}


Table B-2. 618-2 Deep Zone Cleanup Verification Data. (2 pages)

\begin{tabular}{|c|c|c|c|c|c|c|c|c|c|c|c|c|c|c|}
\hline $\begin{array}{c}\text { Sampling } \\
\text { Area }\end{array}$ & $\begin{array}{c}\text { Sample } \\
\text { Number }\end{array}$ & $\begin{array}{c}\text { Sample } \\
\text { Date }\end{array}$ & $\mathbf{m g} / \mathbf{k g}$ & $\mathbf{Q}$ & $\mathbf{P Q L}$ & $\mathbf{m g} / \mathbf{k g}$ & $\mathbf{Q}$ & $\mathbf{P Q L}$ & $\mathbf{m g} / \mathbf{k g}$ & $\mathbf{Q}$ & $\mathbf{P Q L}$ & $\mathbf{m g} / \mathbf{k g}$ & $\mathbf{Q}$ & $\mathrm{PQL}$ \\
\hline $\mathrm{A} 1$ & $\mathrm{~J} 13 \mathrm{DJ} 4$ & $9 / 7 / 06$ & $1.4 \mathrm{E}+00$ & & $1.1 \mathrm{E}+00$ & $5.95 \mathrm{E}+01$ & & $6 \mathrm{E}-02$ & $9 \mathrm{E}-02$ & $\mathrm{U}$ & $9 \mathrm{E}-02$ & $4.2 \mathrm{E}+00$ & $2.1 \mathrm{E}+00$ \\
\hline $\mathrm{A} 3$ & $\mathrm{~J} 13 \mathrm{DJ} 2$ & $9 / 7 / 06$ & $1.1 \mathrm{E}+00$ & $\mathrm{U}$ & $1.1 \mathrm{E}+00$ & $5.85 \mathrm{E}+01$ & & $6 \mathrm{E}-02$ & $9 \mathrm{E}-02$ & $\mathrm{U}$ & $9 \mathrm{E}-02$ & $3.7 \mathrm{E}+00$ & $2.1 \mathrm{E}+00$ \\
\hline $\mathrm{A} 4$ & $\mathrm{~J} 13 \mathrm{D} J 3$ & $9 / 7 / 06$ & $1.5 \mathrm{E}+00$ & & $1.1 \mathrm{E}+00$ & $8.33 \mathrm{E}+01$ & & $6 \mathrm{E}-02$ & $9 \mathrm{E}-02$ & $\mathrm{U}$ & $9 \mathrm{E}-02$ & $6.7 \mathrm{E}+00$ & $2.0 \mathrm{E}-01$ \\
\hline A2 & $\mathrm{J} 13 \mathrm{DJ} 0$ & $9 / 7 / 06$ & $1.1 \mathrm{E}+00$ & $\mathrm{U}$ & $1.1 \mathrm{E}+00$ & $6.35 \mathrm{E}+01$ & & $6 \mathrm{E}-02$ & $9 \mathrm{E}-02$ & $\mathrm{U}$ & $9 \mathrm{E}-02$ & $2.5 \mathrm{E}+00$ & $2.0 \mathrm{E}-01$ \\
\hline $\begin{array}{c}\text { Duplicate } \\
\text { of J13DJ0 }\end{array}$ & J13DJ1 & $9 / 7 / 06$ & $1.1 \mathrm{E}+00$ & $\mathrm{U}$ & $1.1 \mathrm{E}+00$ & $7.37 \mathrm{E}+01$ & & $6 \mathrm{E}-02$ & $9 \mathrm{E}-02$ & $\mathrm{U}$ & $9 \mathrm{E}-02$ & $4.4 \mathrm{E}+00$ & $2.0 \mathrm{E}-01$ \\
\hline $\begin{array}{c}\text { Split of } \\
\text { J13DJ0 }\end{array}$ & J13DK3 & $9 / 7 / 06$ & $1.8 \mathrm{E}+00$ & & $2.8 \mathrm{E}-01$ & $1.85 \mathrm{E}+02$ & & $5.1 \mathrm{E}-01$ & $1.4 \mathrm{E}-01$ & $\mathrm{U}$ & $1.4 \mathrm{E}-01$ & $5.4 \mathrm{E}+00$ & $3.6 \mathrm{E}-01$ \\
\hline
\end{tabular}

\begin{tabular}{|c|c|c|c|c|c|c|c|c|c|c|c|c|c|c|}
\hline \multirow{2}{*}{$\begin{array}{c}\text { Sampling } \\
\text { Area }\end{array}$} & \multirow{2}{*}{$\begin{array}{l}\text { Sample } \\
\text { Number }\end{array}$} & \multirow{2}{*}{$\begin{array}{c}\text { Sample } \\
\text { Date }\end{array}$} & \multicolumn{3}{|c|}{ Lead } & \multicolumn{3}{|c|}{ Selenium } & \multicolumn{3}{|c|}{ Tin } & \multicolumn{3}{|c|}{ Uranium } \\
\hline & & & $\mathrm{mg} / \mathrm{kg}$ & $\mathbf{Q}$ & $P Q L$ & $\mathrm{mg} / \mathrm{kg}$ & $\mathbf{Q}$ & $P Q L$ & $\mathrm{mg} / \mathrm{kg}$ & $\mathbf{Q}$ & $P Q L$ & $\mathrm{mg} / \mathrm{kg}$ & $\mathbf{Q}$ & PQL \\
\hline $\mathrm{A} 1$ & J13DJ4 & $9 / 7 / 06$ & $6.6 \mathrm{E}+00$ & & $3.5 \mathrm{E}-01$ & $7.8 \mathrm{E}-01$ & & $7.6 \mathrm{E}-01$ & $2.8 \mathrm{E}+00$ & $J$ & $1.0 \mathrm{E}+00$ & $4.54 \mathrm{E}+00$ & & $7 \mathrm{E}-03$ \\
\hline$\overline{A 3}$ & J13DJ2 & $9 / 7 / 06$ & $2.7 \mathrm{E}+00$ & & $3.5 \mathrm{E}-01$ & $7.6 \mathrm{E}-01$ & $\mathrm{U}$ & $7.6 \mathrm{E}-01$ & $2.0 E+00$ & $J$ & $1.0 \mathrm{E}+00$ & $1.37 \mathrm{E}+01$ & & $7.2 \mathrm{E}-02$ \\
\hline$\overline{\mathrm{A} 4}$ & $3 \mathrm{DJ} 3$ & $9 / 7 / 06$ & $3.8 \mathrm{E}+00$ & & $3.5 \mathrm{E}+00$ & $7.5 \mathrm{E}-01$ & $U$ & $7.5 \mathrm{E}-01$ & $2.7 \mathrm{E}+00$ & $\mathrm{~J}$ & $1.0 \mathrm{E}+00$ & $5.01 E+02$ & & $7.2 \mathrm{E}-01$ \\
\hline $\bar{A} 2$ & J13DJ0 & $9 / 7 / 06$ & $2.7 \mathrm{E}+00$ & & $3.5 \mathrm{E}+00$ & $7.6 \mathrm{E}-01$ & $\bar{U}$ & $7.6 \mathrm{E}-01$ & $2.5 \mathrm{E}+00$ & $\mathrm{~J}$ & $1.0 \mathrm{E}+00$ & $3.57 \mathrm{E}+01$ & & $7.2 \mathrm{E}-02$ \\
\hline $\begin{array}{l}\text { Duplicate } \\
\text { of } \mathrm{J} 13 \mathrm{DJ} 0\end{array}$ & $J 1$ & 9/ & 3.2 & & $3.5 \mathrm{E}-01$ & 7.6E-01 & U & $7.6 \mathrm{E}-01$ & $2.9 \mathrm{E}+00$ & $\mathrm{~J}$ & $1.0 \mathrm{E}+00$ & $3.70 E+01$ & & 7.2E-02 \\
\hline $\begin{array}{l}\text { Split of } \\
\text { J13DJ0 }\end{array}$ & DK3 & 9/7/06 & 3.5 & & $1.5 \mathrm{E}-01$ & $3.9 \mathrm{E}-01$ & & $1.8 \mathrm{E}-01$ & $8.7 E+00$ & & $2.9 \mathrm{E}+00$ & $8.98 \mathrm{E}+01$ & & 2.04E-02 \\
\hline
\end{tabular}


Table B-2. 618-2 Deep Zone Cleanup Verification Data. (2 pages)

\begin{tabular}{|c|c|c|c|c|c|c|c|c|c|c|c|c|c|c|c|c|c|}
\hline \multirow{2}{*}{$\begin{array}{c}\text { Sampling } \\
\text { Area }\end{array}$} & \multirow{2}{*}{$\begin{array}{l}\text { Sample } \\
\text { Number }\end{array}$} & \multirow{2}{*}{$\begin{array}{c}\text { Sample } \\
\text { Date }\end{array}$} & \multicolumn{3}{|c|}{ Americium-241 } & \multicolumn{3}{|c|}{ Cesium-137 } & \multicolumn{3}{|c|}{ Cobalt-60 } & \multicolumn{3}{|c|}{ Europium-152 } & \multicolumn{3}{|c|}{ Europium-154 } \\
\hline & & & $\mathrm{pCi} / \mathrm{g}$ & $Q$ & MDA & $\mathrm{pCi} / \mathrm{g}$ & $\mathbf{Q}$ & MDA & $\mathrm{pCi} / \mathrm{g}$ & $Q$ & MDA & $\mathrm{pCi} / \mathrm{g}$ & Q & MDA & $\mathrm{pCi} / \mathrm{g}$ & $\mathbf{Q}$ & MDA \\
\hline $\mathrm{A1}$ & J13DJ4 & $9 / 7 / 06$ & $3.08 \mathrm{E}-01$ & & $7.2 E-02$ & $7.86 \mathrm{E}-01$ & & $2.3 \mathrm{E}-02$ & 1.7E-02 & $U$ & 1.7E-02 & $4.7 E-02$ & $U$ & 4.7E-02 & $5.2 E-02$ & $\mathrm{U}$ & $5.2 E-02$ \\
\hline$A 3$ & J13DJ2 & $9 / 7 / 06$ & $2.77 E+00$ & & $7.9 \mathrm{E}-02$ & $5.90 E-02$ & & $4.8 \mathrm{E}-02$ & 3.7E-02 & $\mathrm{U}$ & $3.7 E-02$ & $9.0 \mathrm{E}-02$ & $\mathrm{U}$ & $9.0 \mathrm{E}-02$ & $1.2 \mathrm{E}-01$ & $U$ & $1.2 \mathrm{E}-01$ \\
\hline A4 & J13DJ3 & $9 / 7 / 06$ & $5.45 \mathrm{E}+00$ & & 8.7E-02 & $1.04 E+00$ & & $5.9 \mathrm{E}-02$ & 4.9E-02 & $\mathrm{U}$ & $4.9 E-02$ & 1.6E-01 & $U$ & $1.6 \mathrm{E}-01$ & $1.8 \mathrm{E}-01$ & $U$ & $1.8 \mathrm{E}-01$ \\
\hline $\mathrm{A} 2$ & J13DJ0 & $9 / 7 / 06$ & $7.94 \mathrm{E}+00$ & & $6.7 \mathrm{E}-02$ & $2.09 \mathrm{E}-01$ & & $4.6 \mathrm{E}-02$ & 3.7E-02 & $\mathrm{U}$ & $3.7 \mathrm{E}-02$ & $9.9 E-02$ & $\mathrm{U}$ & $9.9 \mathrm{E}-02$ & $1.2 \mathrm{E}-01$ & $\mathrm{U}$ & $1.2 \mathrm{E}-01$ \\
\hline \begin{tabular}{|c|} 
Duplicate of \\
J13DJ0
\end{tabular} & J13DJ1 & $9 / 7 / 06$ & $6.82 E+00$ & & 4.7E-02 & 3.92E-01 & & 4.6E-02 & $3.2 E-02$ & $U$ & $3.2 E-02$ & $9.2 E-02$ & $U$ & $9.2 \mathrm{E}-02$ & 1.1E-01 & $U$ & 1.1E-01 \\
\hline $\begin{array}{l}\text { Split of } \\
\text { J13DJ0 }\end{array}$ & J13DK3 & $9 / 7 / 06$ & $1.78 E+01$ & & 2.00E-02 & $2.78 \mathrm{E}-01$ & & $1.80 \mathrm{E}-02$ & 4.98E-03 & $U$ & $1.88 \mathrm{E}-02$ & $-1.00 E-02$ & $u$ & 4.12E-02 & $-2.80 E-02$ & $\mathrm{U}$ & $5.74 \mathrm{E}-02$ \\
\hline
\end{tabular}

\begin{tabular}{|c|c|c|c|c|c|c|c|c|c|c|c|c|c|c|c|c|c|}
\hline \multirow{2}{*}{$\begin{array}{c}\text { Sampling } \\
\text { Area }\end{array}$} & \multirow{2}{*}{$\begin{array}{l}\text { Sample } \\
\text { Number }\end{array}$} & \multirow{2}{*}{$\begin{array}{c}\text { Sample } \\
\text { Date }\end{array}$} & \multicolumn{3}{|c|}{ Europium-155 } & \multicolumn{3}{|c|}{ Nickel-63 } & \multicolumn{3}{|c|}{ Tritium } & \multicolumn{3}{|c|}{ Plutonium-238 } & \multicolumn{3}{|c|}{ Plutonium-239/240 } \\
\hline & & & $\mathrm{pCi} / \mathrm{g}$ & $\mathbf{Q}$ & MDA & $\mathrm{pCi} / \mathrm{g}$ & Q & MDA & $\mathrm{pCi} / \mathrm{g}$ & $\mathbf{Q}$ & MDA & $\mathrm{pCi} / \mathrm{g}$ & $\mathbf{Q}$ & MDA & $\mathrm{pCi} / \mathrm{g}$ & $\mathbf{Q}$ & MDA \\
\hline $\mathrm{A1}$ & J13DJ4 & $9 / 7 / 06$ & $4.0 E-02$ & $U$ & $4.0 \mathrm{E}-02$ & $1.72 \mathrm{E}-01$ & $\mathrm{U}$ & $3.8 E+00$ & $7.9 \mathrm{E}-02$ & $U$ & $2.5 E+00$ & $9.8 E-02$ & $\mathrm{U}$ & $3.7 E-01$ & $2.20 E+00$ & & $3.7 E-01$ \\
\hline A3 & J13DJ2 & $9 / 7 / 06$ & $8.6 \mathrm{E}-02$ & $\mathrm{U}$ & $8.6 \mathrm{E}-02$ & $-1.48 E+00$ & $\mathrm{U}$ & $3.5 E+00$ & $6.24 E-01$ & $U$ & $2.3 E+00$ & $1.98 \mathrm{E}-01$ & $U$ & 5.0E-01 & $2.68 \mathrm{E}+01$ & & $5.0 \mathrm{E}-01$ \\
\hline A4 & J13DJ3 & $9 / 7 / 06$ & $3.5 \mathrm{E}-01$ & $U$ & $3.5 \mathrm{E}-01$ & $-4.06 E-01$ & $\mathrm{U}$ & $3.6 \mathrm{E}+00$ & $8.59 \mathrm{E}-01$ & $U$ & $2.3 E+00$ & $1.50 E+00$ & & $4.8 \mathrm{E}-01$ & $9.06 \mathrm{E}+01$ & & $4.8 E-01$ \\
\hline $\mathrm{A} 2$ & J13DJ0 & $9 / 7 / 06$ & $9.6 E-02$ & $U$ & $9.6 \mathrm{E}-02$ & $-4.97 E-01$ & $\mathrm{U}$ & $3.6 \mathrm{E}+00$ & $4.54 \mathrm{E}-01$ & $\mathrm{U}$ & $2.5 E+00$ & $4.17 \mathrm{E}-01$ & $\mathrm{U}$ & $5.3 E-01$ & $5.16 \mathrm{E}+01$ & & $5.3 \mathrm{E}-01$ \\
\hline $\begin{array}{c}\text { Duplicate of } \\
\text { J13DJ0 }\end{array}$ & J13DJ1 & $9 / 7 / 06$ & 8.9E-02 & $U$ & 8.9E-02 & 8.68E-01 & $U$ & $3.6 E+00$ & $5.80 \mathrm{E}-01$ & $U$ & $2.4 \mathrm{E}+00$ & $4.15 \mathrm{E}-01$ & $U$ & $4.5 \mathrm{E}-01$ & $4.55 E+01$ & & $4.5 \mathrm{E}-01$ \\
\hline $\begin{array}{l}\text { Split of } \\
\text { J13DJ0 }\end{array}$ & J13DK3 & $9 / 7 / 06$ & 4.37E-02 & $U$ & $5.46 \mathrm{E}-02$ & $4.44 E+00$ & $\mathrm{U}$ & $6.05 E+00$ & 7.13E-02 & & 3.80E-02 & $1.32 E+00$ & & 2.19E-02 & $9.23 E+01$ & & 2.19E-02 \\
\hline
\end{tabular}

\begin{tabular}{|c|c|c|c|c|c|c|c|c|c|c|c|c|c|c|c|c|c|}
\hline \multirow{2}{*}{$\begin{array}{c}\text { Sampling } \\
\text { Area }\end{array}$} & \multirow{2}{*}{$\begin{array}{l}\text { Sample } \\
\text { Number }\end{array}$} & \multirow{2}{*}{$\begin{array}{c}\text { Sample } \\
\text { Date } \\
\end{array}$} & \multicolumn{3}{|c|}{ Plutonium-241 } & \multicolumn{3}{|c|}{ Strontium-90 } & \multicolumn{3}{|c|}{ Uranium-233/234 } & \multicolumn{3}{|c|}{ Uranium-235 } & \multicolumn{3}{|c|}{ Uranium-238 } \\
\hline & & & $\mathrm{pCi} / \mathrm{g}$ & $\mathbf{Q}$ & MDA & $\mathrm{pCi} / \mathrm{g}$ & Q & MDA & $\mathrm{pCi} / \mathrm{g}$ & $\mathbf{Q}$ & MDA & $\mathrm{pCi} / \mathrm{g}$ & $Q$ & MDA & $\mathrm{pCi} / \mathrm{g}$ & $\mathbf{Q}$ & MDA \\
\hline $\mathrm{A} 1$ & J13DJ4 & $9 / 7 / 06$ & $7.07 E+00$ & & $1.8 \mathrm{E}+01$ & $9.9 E-02$ & $\mathrm{U}$ & $2.2 \mathrm{E}-01$ & $3.26 \mathrm{E}+00$ & & 2.2E-01 & $1.02 \mathrm{E}-01$ & $U$ & 2.6E-01 & $2.87 E+00$ & & 2.2E-01 \\
\hline $\mathrm{A} 3$ & J13DJ2 & $9 / 7 / 06$ & $2.08 \mathrm{E}+01$ & & $2.0 \mathrm{E}+01$ & $1.81 \mathrm{E}+00$ & & $2.2 \mathrm{E}-01$ & $5.53 \mathrm{E}+00$ & & $2.2 E-01$ & $1.02 E-01$ & $U$ & $2.6 \mathrm{E}-01$ & $5.22 \mathrm{E}+00$ & & $2.2 \mathrm{E}-01$ \\
\hline A4 & J13DJ3 & $9 / 7 / 06$ & $4.20 E+01$ & & $2.6 \mathrm{E}+01$ & $1.75 E+00$ & & 1.9E-01 & $1.61 \mathrm{E}+02$ & & $4.8 \mathrm{E}-01$ & $3.35 E+00$ & $\mathrm{U}$ & $5.8 \mathrm{E}+00$ & $1.65 \mathrm{E}+02$ & & $4.6 \mathrm{E}-01$ \\
\hline $\mathrm{A} 2$ & J13DJ0 & $9 / 7 / 06$ & $2.97 E+01$ & & $2.9 \mathrm{E}+01$ & $1.16 \mathrm{E}+01$ & & 2.1E-01 & $1.46 \mathrm{E}+01$ & & 2.9E-01 & 7.76E-01 & & $2.8 \mathrm{E}-01$ & $1.48 \mathrm{E}+01$ & & $2.9 \mathrm{E}-01$ \\
\hline $\begin{array}{c}\text { Duplicate of } \\
\text { J13DJ0 }\end{array}$ & J13DJ1 & $9 / 7 / 06$ & $9.69 E+00$ & $U$ & $2.2 E+01$ & $9.39 \mathrm{E}+00$ & & $2.0 \mathrm{E}-01$ & $1.28 \mathrm{E}+01$ & & $3.0 \mathrm{E}-01$ & 7.61E-01 & & $2.5 \mathrm{E}-01$ & $1.25 E+01$ & & 2.6E-01 \\
\hline
\end{tabular}


Table B-3. $618-2$ Overburden Cleanup Verification Data. (2 pages)

\begin{tabular}{|c|c|c|c|c|c|c|c|c|c|c|c|c|c|c|}
\hline \multirow{2}{*}{$\begin{array}{c}\text { Sampling } \\
\text { Area }\end{array}$} & \multirow{2}{*}{$\begin{array}{l}\text { Sample } \\
\text { Number }\end{array}$} & \multirow{2}{*}{$\begin{array}{c}\text { Sample } \\
\text { Date }\end{array}$} & \multicolumn{3}{|c|}{ Arsenic } & \multicolumn{3}{|c|}{ Barium } & \multicolumn{3}{|c|}{ Cadmium } & \multicolumn{3}{|c|}{ Chromium } \\
\hline & & & $\mathrm{mg} / \mathrm{kg}$ & $\mathbf{Q}$ & $\overline{P Q L}$ & $\mathrm{mg} / \mathrm{kg}$ & Q & $\overline{P Q L}$ & $\mathrm{mg} / \mathrm{kg}$ & $\mathbf{Q}$ & $\overline{P Q L}$ & $\mathrm{mg} / \mathrm{kg}$ & $\mathbf{Q}$ & $\overline{P Q L}$ \\
\hline $\mathrm{A} 1$ & J13DF9 & $9 / 7 / 06$ & $2.7 E+00$ & & $1.2 E+00$ & $6.42 \mathrm{E}+01$ & & $6 \mathrm{E}-02$ & $9 \mathrm{E}-02$ & $U$ & $9 \mathrm{E}-02$ & $6.1 E+00$ & & 2.0E-01 \\
\hline $\mathrm{A3}$ & $\mathrm{J} 13 \mathrm{DH} 2$ & $9 / 7 / 06$ & $1.8 \mathrm{E}+00$ & & $1.1 \mathrm{E}+00$ & $7.47 E+01$ & & $6 \mathrm{E}-02$ & $9 \mathrm{E}-02$ & $U$ & $9 \mathrm{E}-02$ & $5.1 E+00$ & & 2.0E-01 \\
\hline A4 & J13DH3 & $9 / 7 / 06$ & $2.4 \mathrm{E}+00$ & & $1.1 \mathrm{E}+00$ & $6.29 E+01$ & & $6 \mathrm{E}-02$ & $9 \mathrm{E}-02$ & $U$ & $9 \mathrm{E}-02$ & $5.2 E+00$ & & 2.0E-01 \\
\hline $\mathrm{A} 2$ & J13DH0 & $9 / 7 / 06$ & $1.5 \mathrm{E}+00$ & & $1.1 \mathrm{E}+00$ & $6.22 E+01$ & & $6 \mathrm{E}-02$ & $9 \mathrm{E}-02$ & U & $9 \mathrm{E}-02$ & $5.3 E+00$ & & 2.0E-01 \\
\hline $\begin{array}{c}\text { Duplicate } \\
\text { of } \mathrm{J} 13 \mathrm{DHO}\end{array}$ & J13DH1 & $9 / 7 / 06$ & 0 & & 0 & 01 & & 6E-02 & $9 \mathrm{E}-02$ & $U$ & $\mathrm{E}-02$ & -00 & & -01 \\
\hline $\begin{array}{l}\text { Split of } \\
\mathrm{J} 13 \mathrm{DHO} O\end{array}$ & $\mathrm{~J} 13 \mathrm{DH} 4$ & 9/7/06 & $2.0 \mathrm{E}+00$ & & 2.8E-01 & $8.41 E+01$ & & $5.0 \mathrm{E}-01$ & $1.4 \mathrm{E}-01$ & $U$ & 1.4E-01 & $7.0 \mathrm{E}+00$ & & $3.6 \mathrm{E}-01$ \\
\hline
\end{tabular}

\begin{tabular}{|c|c|c|c|c|c|c|c|c|c|c|c|c|c|c|}
\hline \multirow{2}{*}{$\begin{array}{c}\text { Sampling } \\
\text { Area }\end{array}$} & \multirow{2}{*}{$\begin{array}{l}\text { Sample } \\
\text { Number }\end{array}$} & \multirow{2}{*}{$\begin{array}{c}\text { Sample } \\
\text { Date }\end{array}$} & \multicolumn{3}{|c|}{ Lead } & \multicolumn{3}{|c|}{ Selenium } & \multicolumn{3}{|c|}{ Tin } & \multicolumn{3}{|c|}{ Uranium } \\
\hline & & & $\mathrm{mg} / \mathrm{kg}$ & $\mathbf{Q}$ & PQL & $\mathrm{mg} / \mathrm{kg}$ & Q & $\overline{P Q L}$ & $\mathrm{mg} / \mathrm{kg}$ & $\mathbf{Q}$ & $\overline{\mathrm{PQL}}$ & $\mathrm{mg} / \mathrm{kg}$ & $\mathbf{Q}$ & $\overline{P Q L}$ \\
\hline$\overline{A 1}$ & J13DF9 & $9 / 7 / 06$ & $4.1 \mathrm{E}+00$ & & $3.8 \mathrm{E}-01$ & $8.2 \mathrm{E}-01$ & $\mathrm{U}$ & $8.2 \mathrm{E}-01$ & $2.7 \mathrm{E}+00$ & $J$ & $1.1 \mathrm{E}+00$ & $1.14 \mathrm{E}+00$ & & $7 \mathrm{E}-03$ \\
\hline $\mathrm{A} 3$ & $\mathrm{~J} 13 \mathrm{DH} 2$ & $9 / 7 / 06$ & $4.3 \mathrm{E}+00$ & & $3.5 \mathrm{E}-01$ & $1.0 \mathrm{E}+00$ & & $7.6 \mathrm{E}-01$ & $1.6 \mathrm{E}+00$ & $J$ & $1.0 \mathrm{E}+00$ & $9.15 E-01$ & & $7 \mathrm{E}-03$ \\
\hline A4 & $\mathrm{J} 13 \mathrm{DH} 3$ & $9 / 7 / 06$ & $3.4 \mathrm{E}+00$ & & $3.5 \mathrm{E}-01$ & $7.5 \mathrm{E}-01$ & $\mathrm{U}$ & $7.5 \mathrm{E}-01$ & $2.0 E+00$ & $\mathrm{~J}$ & $1.0 E+00$ & $8.72 \mathrm{E}-01$ & & $7 \mathrm{E}-03$ \\
\hline A2 & $\mathrm{J} 13 \mathrm{DHO}$ & $9 / 7 / 06$ & $3.5 E+00$ & & $3.5 \mathrm{E}-01$ & $7.6 \mathrm{E}-01$ & $\mathrm{U}$ & $7.6 \mathrm{E}-01$ & $1.0 \mathrm{E}+00$ & $\mathrm{U}$ & $1.0 \mathrm{E}+00$ & 9.72E-01 & & $7 E-03$ \\
\hline $\begin{array}{c}\text { Duplicate } \\
\text { of } \mathrm{J} 13 \mathrm{DH} 0\end{array}$ & J13DH1 & 9/7/06 & $4.3 E+00$ & & $3.5 \mathrm{E}-01$ & 7.5E-01 & $u$ & 7.5E-01 & $2.2 E+00$ & $J$ & $1.0 \mathrm{E}+00$ & 8.91E-01 & & 7E-03 \\
\hline $\begin{array}{l}\text { Split of } \\
\text { J13DHO }\end{array}$ & $\mathrm{J} 13 \mathrm{DH} 4$ & $9 / 7 / 06$ & $3.2 E+00$ & & $1.5 \mathrm{E}-01$ & 5.5E-01 & & 1.7E-01 & $2.8 E+00$ & U & $2.8 E+00$ & $2.69 \mathrm{E}+00$ & & 2.10E-02 \\
\hline
\end{tabular}


Table B-3. 618-2 Overburden Cleanup Verification Data. (2 pages)

\begin{tabular}{|c|c|c|c|c|c|c|c|c|c|c|c|c|c|c|c|c|c|}
\hline \multirow{2}{*}{$\begin{array}{c}\text { Sampling } \\
\text { Area }\end{array}$} & \multirow{2}{*}{$\begin{array}{l}\text { Sample } \\
\text { Number }\end{array}$} & \multirow{2}{*}{$\begin{array}{c}\text { Sample } \\
\text { Date }\end{array}$} & \multicolumn{3}{|c|}{ Americium-241 } & \multicolumn{3}{|c|}{ Cesium-137 } & \multicolumn{3}{|c|}{ Cobalt-60 } & \multicolumn{3}{|c|}{ Europium-152 } & \multicolumn{3}{|c|}{ Europium-154 } \\
\hline & & & $\mathrm{pCi} / \mathrm{g}$ & $Q$ & MDA & $\mathrm{pCi} / \mathrm{g}$ & $\mathbf{Q}$ & MDA & $\mathrm{pCi} / \mathrm{g}$ & $Q$ & MDA & $\mathrm{pCi} / \mathrm{g}$ & $\mathrm{Q}$ & MDA & $\mathrm{pCi} / \mathrm{g}$ & $\mathbf{Q}$ & MDA \\
\hline $\mathrm{A} 1$ & J13DF9 & $9 / 7 / 06$ & $-6.0 \mathrm{E}-03$ & $U$ & $6.9 \mathrm{E}-02$ & $5.8 \mathrm{E}-02$ & & $3.9 \mathrm{E}-02$ & $3.3 \mathrm{E}-02$ & $U$ & $3.3 \mathrm{E}-02$ & $9.0 \mathrm{E}-02$ & $\mathrm{U}$ & $9.0 \mathrm{E}-02$ & $1.1 \mathrm{E}-01$ & $U$ & 1.1E-01 \\
\hline $\mathrm{A} 3$ & $\mathrm{~J} 13 \mathrm{DH} 2$ & $9 / 7 / 06$ & $3.5 \mathrm{E}-02$ & $\mathrm{U}$ & $6.2 \mathrm{E}-02$ & $3.6 \mathrm{E}-02$ & $\mathrm{U}$ & $3.9 \mathrm{E}-02$ & 4.0E-02 & $U$ & $4.0 \mathrm{E}-02$ & $1.1 \mathrm{E}-01$ & $U$ & $1.1 \mathrm{E}-01$ & 1.4E-01 & $\mathrm{U}$ & 1.4E-01 \\
\hline A4 & $\mathrm{J13DH3}$ & $9 / 7 / 06$ & $-1.2 E-02$ & $\mathrm{U}$ & $8.2 E-02$ & $4.0 E-02$ & & $3.4 \mathrm{E}-02$ & $2.9 \mathrm{E}-02$ & $U$ & 2.9E-02 & $7.4 \mathrm{E}-02$ & $U$ & $7.4 \mathrm{E}-02$ & $9.9 \mathrm{E}-02$ & $U$ & 9.9E-02 \\
\hline $\mathrm{A} 2$ & $\mathrm{~J} 13 \mathrm{DHO}$ & $9 / 7 / 06$ & $2.0 \mathrm{E}-02$ & $U$ & $6.5 \mathrm{E}-02$ & $4.2 \mathrm{E}-02$ & $U$ & $4.2 \mathrm{E}-02$ & $3.8 \mathrm{E}-02$ & $U$ & $3.8 \mathrm{E}-02$ & $9.3 \mathrm{E}-02$ & $\mathrm{U}$ & $9.3 E-02$ & $1.2 \mathrm{E}-01$ & $\mathrm{U}$ & $1.2 \mathrm{E}-01$ \\
\hline $\begin{array}{l}\text { Duplicate } \\
\text { of } \mathrm{J} 13 \mathrm{DH} 0\end{array}$ & J13DH1 & $9 / 7 / 06$ & $-5.0 E-03$ & $U$ & 7.0E-02 & 4.8E-02 & & 4.8E-02 & 4.9E-02 & $U$ & 4.9E-02 & 1.2E-01 & $U$ & $1.2 E-01$ & 1.6E-01 & $U$ & $1.6 \mathrm{E}-01$ \\
\hline $\begin{array}{c}\text { Split of } \\
J 13 \mathrm{DHO}\end{array}$ & $\mathrm{J} 13 \mathrm{DH} 4$ & $9 / 7 / 06$ & 2.97E-02 & & 2.03E-02 & $1.55 E-02$ & & $1.50 \mathrm{E}-02$ & $1.98 E-03$ & $U$ & $1.65 E-02$ & $-1.99 E-02$ & $U$ & $3.28 \mathrm{E}-02$ & 2.36E-02 & $U$ & 5.37E-02 \\
\hline
\end{tabular}

\begin{tabular}{|c|c|c|c|c|c|c|c|c|c|c|c|c|c|c|c|c|c|}
\hline \multirow{2}{*}{$\begin{array}{c}\text { Sampling } \\
\text { Area }\end{array}$} & \multirow{2}{*}{$\begin{array}{l}\text { Sample } \\
\text { Number }\end{array}$} & \multirow{2}{*}{$\begin{array}{c}\text { Sample } \\
\text { Date }\end{array}$} & \multicolumn{3}{|c|}{ Europium-155 } & \multicolumn{3}{|c|}{ Nickel-63 } & \multicolumn{3}{|c|}{ Tritium } & \multicolumn{3}{|c|}{ Plutonium-238 } & \multicolumn{3}{|c|}{ Plutonium-239/240 } \\
\hline & & & $\mathrm{pCi} / \mathrm{g}$ & Q & MDA & $\mathrm{pCi} / \mathrm{g}$ & $\mathbf{Q}$ & MDA & $\mathrm{pCi} / \mathrm{g}$ & Q & MDA & $\mathrm{pCi} / \mathrm{g}$ & Q & MDA & $\mathrm{pCi} / \mathrm{g}$ & $\mathbf{Q}$ & MDA \\
\hline$\overline{A 1}$ & J13DF9 & $9 / 7 / 06$ & $8.9 \mathrm{E}-02$ & $U$ & $8.9 E-02$ & $-6.92 E-01$ & $u$ & $3.8 \mathrm{E}+00$ & $7.23 \mathrm{E}-01$ & U & $2.5 E+00$ & $-6.2 E-02$ & $\mathrm{U}$ & $4.7 \mathrm{E}-01$ & $6.2 E-02$ & $U$ & $4.7 E-01$ \\
\hline A3 & $\mathrm{J} 13 \mathrm{DH} 2$ & $9 / 7 / 06$ & $1.4 \mathrm{E}-01$ & U & $1.4 \mathrm{E}-01$ & $1.02 E+00$ & $U$ & $4.1 E+00$ & $1.23 \mathrm{E}-01$ & U & $2.6 E+00$ & 0 & $\mathrm{U}$ & $4.9 \mathrm{E}-01$ & $6.5 \mathrm{E}-02$ & $\mathrm{U}$ & $4.9 \mathrm{E}-01$ \\
\hline A4 & $\mathrm{J} 13 \mathrm{DH} 3$ & $9 / 7 / 06$ & $6.6 \mathrm{E}-02$ & U & $6.6 E-02$ & $2.43 \mathrm{E}-01$ & $U$ & $4.3 E+00$ & $9.62 \mathrm{E}-01$ & U & $2.4 E+00$ & 0 & $U$ & $5.2 E-01$ & 0 & $U$ & $5.2 \mathrm{E}-01$ \\
\hline $\mathrm{A} 2$ & $\mathrm{J13DHO}$ & $9 / 7 / 06$ & $8.4 \mathrm{E}-02$ & U & $8.4 \mathrm{E}-02$ & $-7.17 \mathrm{E}-01$ & $\mathrm{U}$ & $3.7 \mathrm{E}+00$ & $3.49 \mathrm{E}-01$ & $U$ & $2.6 E+00$ & $1.26 \mathrm{E}-01$ & $\mathrm{U}$ & $4.8 \mathrm{E}-01$ & 0 & $\mathrm{U}$ & $4.8 \mathrm{E}-01$ \\
\hline $\begin{array}{l}\text { Duplicate } \\
\text { of } \mathrm{J} 13 \mathrm{DHO}\end{array}$ & J13DH1 & $9 / 7 / 06$ & $1.1 \mathrm{E}-01$ & $\mathrm{U}$ & 1.1E-01 & $-1.15 E+00$ & $U$ & $4.0 E+00$ & 5.62E-01 & $U$ & $2.6 E+00$ & 0 & $U$ & 5.0E-01 & 1.97E-01 & $U$ & 5.0E-01 \\
\hline $\begin{array}{c}\text { Split of } \\
\text { J13DH0 }\end{array}$ & J13DH4 & 9/7/06 & 1.92E-02 & $U$ & 4.05E-02 & $1.82 E+00$ & $U$ & $5.96 E+00$ & 3.38E-02 & $U$ & $4.15 \mathrm{E}-02$ & 0 & 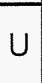 & $1.79 \mathrm{E}-02$ & $-1.32 E-03$ & $U$ & 3.16E-02 \\
\hline
\end{tabular}

\begin{tabular}{|c|c|c|c|c|c|c|c|c|c|c|c|c|c|c|c|c|c|}
\hline \multirow{2}{*}{$\begin{array}{c}\text { Sampling } \\
\text { Area }\end{array}$} & \multirow{2}{*}{$\begin{array}{l}\text { Sample } \\
\text { Number }\end{array}$} & \multirow{2}{*}{$\begin{array}{c}\text { Sample } \\
\text { Date }\end{array}$} & \multicolumn{3}{|c|}{ Plutonium-241 } & \multicolumn{3}{|c|}{ Strontium-90 } & \multicolumn{3}{|c|}{ Uranium-233/234 } & \multicolumn{3}{|c|}{ Uranium-235 } & \multicolumn{3}{|c|}{ Uranium-238 } \\
\hline & & & $\mathrm{pCi} / \mathrm{g}$ & $\mathrm{Q}$ & MDA & $\mathrm{pCi} / \mathrm{g}$ & $\mathbf{Q}$ & MDA & $\mathrm{pCi} / \mathrm{g}$ & $\mathbf{Q}$ & MDA & $\mathrm{pCi} / \mathrm{g}$ & $\mathbf{Q}$ & MDA & $\mathrm{pCi} / \mathrm{g}$ & $\mathbf{Q}$ & MDA \\
\hline$\overline{A 1}$ & J13DF9 & $9 / 7 / 06$ & $-7.94 \mathrm{E}+00$ & U & $2.2 E+01$ & $5.1 \mathrm{E}-02$ & $U$ & $2.6 \mathrm{E}-01$ & $5.42 E-01$ & & 1.9E-01 & $3.0 \mathrm{E}-02$ & $U$ & $2.3 E-01$ & $4.44 \mathrm{E}-01$ & & $1.9 \mathrm{E}-01$ \\
\hline$\overline{A 3}$ & $\mathrm{~J} 13 \mathrm{DH} 2$ & $9 / 7 / 06$ & $-5.23 E+00$ & U & $2.4 \mathrm{E}+01$ & $7.0 \mathrm{E}-02$ & $\mathrm{U}$ & $2.5 \mathrm{E}-01$ & $4.38 \mathrm{E}-01$ & & $2.1 \mathrm{E}-01$ & $3.3 \mathrm{E}-02$ & $U$ & $2.5 \mathrm{E}-01$ & 3.56E-01 & & $2.1 \mathrm{E}-01$ \\
\hline $\mathrm{A} 4$ & $\mathrm{J13 \textrm {DH } 3}$ & $9 / 7 / 06$ & $-4.35 E+00$ & U & $2.5 E+01$ & $-2.4 \mathrm{E}-02$ & $\mathrm{U}$ & $2.4 \mathrm{E}-01$ & $5.86 E-01$ & & $2.1 \mathrm{E}-01$ & $3.4 \mathrm{E}-02$ & $U$ & $2.6 \mathrm{E}-01$ & 4.75E-01 & & $2.1 \mathrm{E}-01$ \\
\hline $\mathrm{A} 2$ & $\mathrm{J13 \textrm {DHO }}$ & $9 / 7 / 06$ & $4.63 E+00$ & $U$ & $2.4 \mathrm{E}+01$ & $-1.2 \mathrm{E}-02$ & $\underline{U}$ & $2.5 \mathrm{E}-01$ & $6.44 \mathrm{E}-01$ & & $2.1 \mathrm{E}-01$ & 9.7E-02 & $U$ & $2.5 \mathrm{E}-01$ & $5.10 E-01$ & & $2.1 \mathrm{E}-01$ \\
\hline $\begin{array}{l}\text { Duplicate } \\
\text { of } \mathrm{J} 13 \mathrm{DHO}\end{array}$ & J13DH1 & $9 / 7 / 06$ & $4.82 E+00$ & $U$ & $2.8 E+01$ & $-9.7 E-02$ & $U$ & $2.5 \mathrm{E}-01$ & 5.37E-01 & & 2.1E-01 & 0 & $U$ & $2.5 \mathrm{E}-01$ & 5.91E-01 & & 2.1E-01 \\
\hline $\begin{array}{l}\text { Split of } \\
\mathrm{J} 13 \mathrm{DHO}\end{array}$ & J13DH4 & $9 / 7 / 06$ & $3.28 \mathrm{E}-01$ & $U$ & $1.50 E+00$ & 8.98E-02 & $U$ & $1.20 \mathrm{E}-01$ & $1.02 \mathrm{E}+00$ & & $3.74 \mathrm{E}-02$ & 2.97E-02 & & 2.43E-02 & 7.67E-01 & & 2.43E-02 \\
\hline
\end{tabular}


Table B-4. 618-2, 618-3, 618-8 Staging Pile/Decon Pad Cleanup Verification Data. (5 pages)

\begin{tabular}{|c|c|c|c|c|c|c|c|c|c|c|c|c|c|c|c|}
\hline \multirow{2}{*}{$\begin{array}{l}\text { Sampling } \\
\text { number }\end{array}$} & \multirow{2}{*}{$\begin{array}{l}\text { Sample } \\
\text { Number }\end{array}$} & \multirow{2}{*}{$\begin{array}{c}\text { Sample } \\
\text { Date }\end{array}$} & \multicolumn{3}{|c|}{ Arsenic } & \multicolumn{3}{|c|}{ Barium } & \multicolumn{3}{|c|}{ Cadmium } & \multicolumn{2}{|c|}{ Chromium } & \multicolumn{2}{|c|}{ Lead } \\
\hline & & & $\mathrm{mg} / \mathrm{kg}$ & \begin{tabular}{|l|l} 
Q \\
\end{tabular} & $\overline{P Q L}$ & $\mathrm{mg} / \mathrm{kg}$ & Q & $\mathrm{PQL}$ & $\mathrm{mg} / \mathrm{kg}$ & Q & $\overline{P Q L}$ & $\mathrm{mg} / \mathrm{kg}$ & $\mathrm{PQL}$ & $\mathrm{mg} / \mathrm{kg}$ & $P Q L$ \\
\hline B5 & J13D61 & $9 / 6 / 06$ & $2.9 E+00$ & U & $2.9 \mathrm{E}+00$ & $7.19 \mathrm{E}+01$ & & $5.73 \mathrm{E}+01$ & $1.4 \mathrm{E}+00$ & U & $1.4 \mathrm{E}+00$ & $6.1 \mathrm{E}+00$ & $2.9 \mathrm{E}+00$ & $3.6 \mathrm{E}+00$ & $8.6 \mathrm{E}-01$ \\
\hline $\mathrm{B} 6$ & J13D60 & $9 / 6 / 06$ & $2.8 \mathrm{E}+00$ & $\mathrm{U}$ & $2.8 \mathrm{E}+00$ & $5.94 \mathrm{E}+01$ & & $5.69 \mathrm{E}+01$ & $1.4 \mathrm{E}+00$ & $U$ & $1.4 \mathrm{E}+00$ & $5.9 \mathrm{E}+00$ & $2.8 \mathrm{E}+00$ & $3.4 \mathrm{E}+00$ & $8.5 \mathrm{E}-01$ \\
\hline B7 & J13D63 & $9 / 6 / 06$ & $2.9 E+00$ & $\mathrm{U}$ & $2.9 E+00$ & $6.24 \mathrm{E}+01$ & & $5.72 E+01$ & $1.4 \mathrm{E}+00$ & $\mathrm{U}$ & $1.4 \mathrm{E}+00$ & $5.0 \mathrm{E}+00$ & $2.9 \mathrm{E}+00$ & $3.7 \mathrm{E}+00$ & $8.6 \mathrm{E}-01$ \\
\hline B8 & J13D64 & $9 / 6 / 06$ & $2.9 \mathrm{E}+00$ & $\mathrm{U}$ & $2.9 E+00$ & $5.91 \mathrm{E}+01$ & & $5.72 E+01$ & $1.4 \mathrm{E}+00$ & $U$ & $1.4 \mathrm{E}+00$ & $4.5 \mathrm{E}+00$ & $2.9 \mathrm{E}+00$ & $2.8 \mathrm{E}+00$ & $8.6 \mathrm{E}-01$ \\
\hline $\mathrm{C9}$ & J13D65 & $9 / 6 / 06$ & $2.8 \mathrm{E}+00$ & $\mathrm{U}$ & $2.8 \mathrm{E}+00$ & $6.54 \mathrm{E}+01$ & & $5.66 \mathrm{E}+01$ & $1.4 \mathrm{E}+00$ & $\mathrm{U}$ & $1.4 \mathrm{E}+00$ & $6.8 \mathrm{E}+00$ & $2.8 \mathrm{E}+00$ & $3.5 \mathrm{E}+00$ & $8.5 \mathrm{E}-01$ \\
\hline $\mathrm{C} 10$ & J13D66 & $9 / 6 / 06$ & $3.0 \mathrm{E}+00$ & & $2.8 \mathrm{E}+00$ & $6.03 E+01$ & & $5.68 \mathrm{E}+01$ & $1.4 \mathrm{E}+00$ & U & $1.4 \mathrm{E}+00$ & $5.8 \mathrm{E}+00$ & $2.8 \mathrm{E}+00$ & $3.4 \mathrm{E}+00$ & $8.5 \mathrm{E}-01$ \\
\hline $\mathrm{C} 1$ & J13D67 & $9 / 6 / 06$ & $2.8 \mathrm{E}+00$ & $\mathrm{U}$ & $2.8 \mathrm{E}+00$ & $6.62 \mathrm{E}+01$ & & $5.68 \mathrm{E}+01$ & $1.4 \mathrm{E}+00$ & U & $1.4 \mathrm{E}+00$ & $6.9 \mathrm{E}+00$ & $2.8 \mathrm{E}+00$ & $4.0 \mathrm{E}+00$ & $8.5 \mathrm{E}-01$ \\
\hline $\mathrm{C} 2$ & J13D68 & $9 / 6 / 06$ & $2.8 \mathrm{E}+00$ & & $2.8 \mathrm{E}+00$ & $6.86 \mathrm{E}+01$ & & $5.64 \mathrm{E}+01$ & $1.4 \mathrm{E}+00$ & $U$ & $1.4 \mathrm{E}+00$ & $6.0 \mathrm{E}+00$ & $2.8 \mathrm{E}+00$ & $4.1 \mathrm{E}+00$ & $8.5 \mathrm{E}-01$ \\
\hline D3 & J13D69 & 9/6/06 & $3.4 \mathrm{E}+00$ & & $2.8 \mathrm{E}+00$ & $8.21 \mathrm{E}+01$ & & $5.66 \mathrm{E}+01$ & $1.4 \mathrm{E}+00$ & $U$ & $1.4 \mathrm{E}+00$ & $7.8 \mathrm{E}+00$ & $2.8 \mathrm{E}+00$ & $4.7 \mathrm{E}+00$ & 8.5E-01 \\
\hline $\mathrm{D} 4$ & J13D70 & $9 / 6 / 06$ & $2.9 \mathrm{E}+00$ & U & $2.9 \mathrm{E}+00$ & $6.09 \mathrm{E}+01$ & & $5.71 \mathrm{E}+01$ & $1.4 \mathrm{E}+00$ & $U$ & $1.4 \mathrm{E}+00$ & $6.1 \mathrm{E}+00$ & $2.9 \mathrm{E}+00$ & $3.6 \mathrm{E}+00$ & $8.6 \mathrm{E}-01$ \\
\hline D5 & J13D71 & $9 / 6 / 06$ & $3.1 \mathrm{E}+00$ & & $2.9 \mathrm{E}+00$ & $6.92 \mathrm{E}+01$ & & $5.74 \mathrm{E}+01$ & $1.4 \mathrm{E}+00$ & U & $1.4 \mathrm{E}+00$ & $5.9 \mathrm{E}+00$ & $2.9 \mathrm{E}+00$ & $5.1 \mathrm{E}+00$ & $8.6 \mathrm{E}-01$ \\
\hline $\mathrm{D} 6$ & J13D72 & $9 / 6 / 06$ & $2.9 \mathrm{E}+00$ & U & $2.9 \mathrm{E}+00$ & $7.37 \mathrm{E}+01$ & & $5.72 \mathrm{E}+01$ & $1.4 \mathrm{E}+00$ & $\mathrm{U}$ & $1.4 \mathrm{E}+00$ & $5.4 \mathrm{E}+00$ & $2.9 \mathrm{E}+00$ & $4.0 \mathrm{E}+00$ & $8.6 \mathrm{E}-01$ \\
\hline $\mathrm{A} 1$ & J13D73 & $9 / 6 / 06$ & $2.8 \mathrm{E}+00$ & U & $2.8 \mathrm{E}+00$ & $5.68 \mathrm{E}+01$ & & $5.66 \mathrm{E}+01$ & $1.4 \mathrm{E}+00$ & $\mathrm{U}$ & $1.4 \mathrm{E}+00$ & $5.9 \mathrm{E}+00$ & $2.8 \mathrm{E}+00$ & $3.3 \mathrm{E}+00$ & $8.5 \mathrm{E}-01$ \\
\hline A3 & J13D75 & $9 / 6 / 06$ & $2.8 \mathrm{E}+00$ & $U$ & $2.8 \mathrm{E}+00$ & $5.78 \mathrm{E}+01$ & & $5.66 \mathrm{E}+01$ & $1.4 \mathrm{E}+00$ & $\mathrm{U}$ & $1.4 \mathrm{E}+00$ & $6.5 \mathrm{E}+00$ & $2.8 \mathrm{E}+00$ & $4.0 \mathrm{E}+00$ & $8.5 \mathrm{E}-01$ \\
\hline A4 & J13D62 & $9 / 6 / 06$ & $3.0 \mathrm{E}+00$ & & $2.9 \mathrm{E}+00$ & $6.28 \mathrm{E}+01$ & & $5.73 \mathrm{E}+01$ & $1.4 \mathrm{E}+00$ & U & $1.4 \mathrm{E}+00$ & $5.9 \mathrm{E}+00$ & $2.9 \mathrm{E}+00$ & $3.6 \mathrm{E}+00$ & $8.6 \mathrm{E}-01$ \\
\hline $\mathrm{A} 2$ & $\mathrm{~J} 13 \mathrm{D} 74$ & $9 / 6 / 06$ & $2.9 \mathrm{E}+00$ & U & $2.9 \mathrm{E}+00$ & $5.75 \mathrm{E}+01$ & $U$ & $5.75 \mathrm{E}+01$ & $1.4 \mathrm{E}+00$ & $U$ & $1.4 \mathrm{E}+00$ & $5.4 \mathrm{E}+00$ & $2.9 \mathrm{E}+00$ & $3.0 \mathrm{E}+00$ & $8.6 \mathrm{E}-01$ \\
\hline $\begin{array}{l}\text { Duplicate } \\
\text { of J13D74 }\end{array}$ & J13D59 & $9 / 6 / 06$ & $3.0 E+00$ & & $2.9 \mathrm{E}+00$ & $5.74 \mathrm{E}+01$ & $U$ & $5.74 \mathrm{E}+01$ & $1.4 \mathrm{E}+00$ & $U$ & $1.4 \mathrm{E}+00$ & $6.8 \mathrm{E}+00$ & $2.9 \mathrm{E}+00$ & $3.5 \mathrm{E}+00$ & 8.6E-01 \\
\hline $\begin{array}{c}\text { Split of } \\
\text { J13D74 }\end{array}$ & J13D76 & $9 / 6 / 06$ & $4.6 E+00$ & & 2.8E-01 & $7.27 \mathrm{E}+01$ & & $5.1 \mathrm{E}-01$ & 1.4E-01 & $U$ & $1.4 \mathrm{E}-01$ & $9.4 \mathrm{E}+00$ & 3.6E-01 & +00 & $1.5 \mathrm{E}-01$ \\
\hline
\end{tabular}


Table B-4. 618-2, 618-3, 618-8 Staging Pile/Decon Pad Cleanup Verification Data. (5 pages)

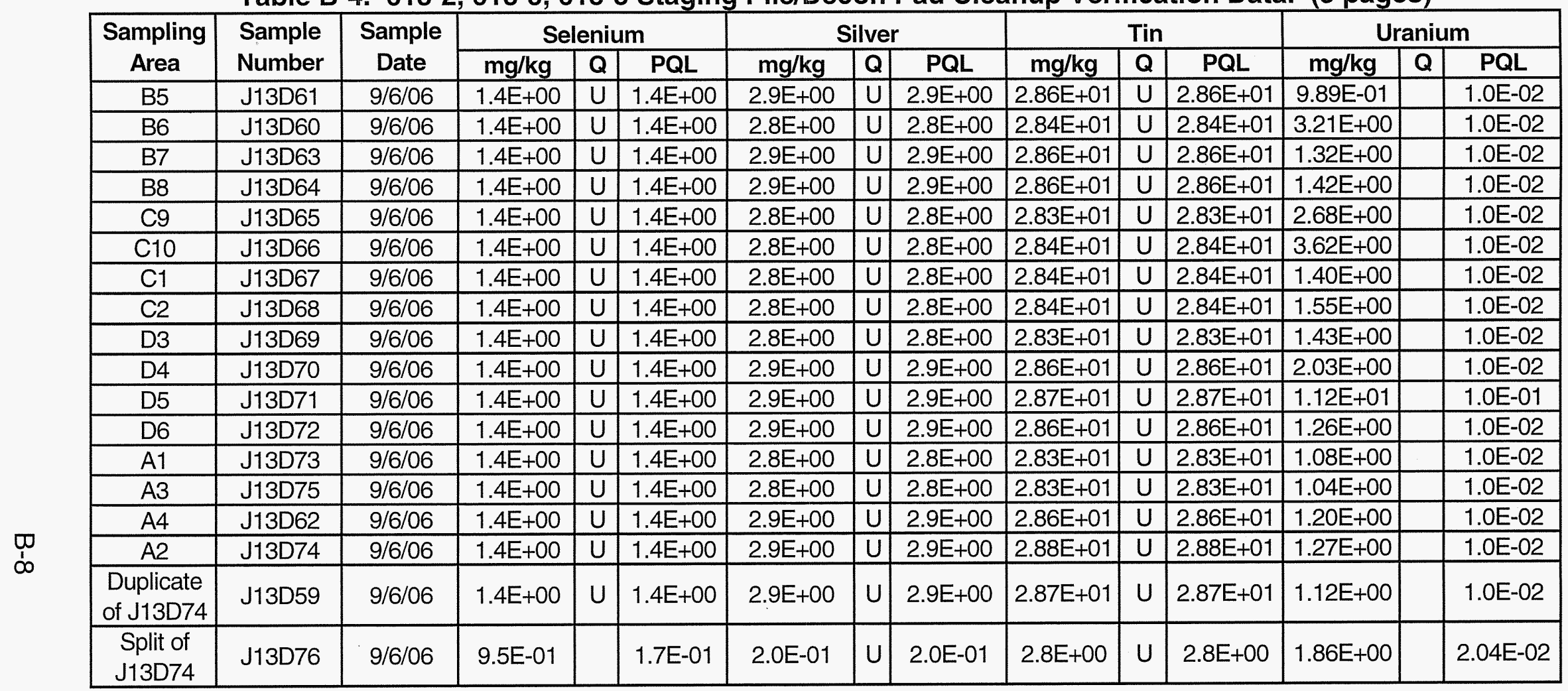


Table B-4. 618-2, 618-3, 618-8 Staging Pile/Decon Pad Cleanup Verification Data. (5 pages)

\begin{tabular}{|c|c|c|c|c|c|c|c|c|c|c|c|c|c|c|c|c|c|}
\hline \multirow{2}{*}{$\begin{array}{c}\text { Sampling } \\
\text { Area }\end{array}$} & \multirow{2}{*}{$\begin{array}{l}\text { Sample } \\
\text { Number }\end{array}$} & \multirow{2}{*}{$\begin{array}{c}\text { Sample } \\
\text { Date }\end{array}$} & \multicolumn{3}{|c|}{ Americium-241 } & \multicolumn{3}{|c|}{ Cesium-137 } & \multicolumn{3}{|c|}{ Cobalt-60 } & \multicolumn{3}{|c|}{ Europium-152 } & \multicolumn{3}{|c|}{ Europium-154 } \\
\hline & & & $\mathrm{pCi} / \mathrm{g}$ & Q & MDA & $\mathrm{pCi} / \mathrm{g}$ & $\mathbf{Q}$ & MDA & $\mathrm{pCi} / \mathrm{g}$ & $\mathbf{Q}$ & MDA & $\mathrm{pCi} / \mathrm{g}$ & $\mathbf{Q}$ & MDA & $\mathrm{pCi} / \mathrm{g}$ & $Q$ & MDA \\
\hline B5 & J13D61 & $9 / 6 / 06$ & $-1.5 \mathrm{E}-02$ & $U$ & $\begin{array}{ll}6.1 \mathrm{E}-02 \\
\end{array}$ & $4.6 \mathrm{E}-02$ & $\bar{U}$ & $4.6 \mathrm{E}-02$ & $4.0 \mathrm{E}-02$ & $U$ & $4.0 \mathrm{E}-02$ & $1.1 \mathrm{E}-01$ & $\bar{U}$ & $1.1 \mathrm{E}-01$ & $1.4 \mathrm{E}-01$ & $U$ & $1.4 \mathrm{E}-01$ \\
\hline$B 6$ & J13D60 & $9 / 6 / 06$ & $4.5 \mathrm{E}-02$ & $U$ & $6.4 \mathrm{E}-02$ & $4.4 \mathrm{E}-02$ & $\bar{U}$ & $4.4 \mathrm{E}-02$ & $4.3 \mathrm{E}-02$ & $U$ & $4.3 \mathrm{E}-02$ & $1.1 \mathrm{E}-01$ & $\bar{U}$ & $1.1 \mathrm{E}-01$ & $1.4 \mathrm{E}-01$ & $U$ & $1.4 \mathrm{E}-01$ \\
\hline B7 & J13D63 & $9 / 6 / 06$ & $-6.0 \mathrm{E}-03$ & $U$ & $6.1 \mathrm{E}-02$ & $3.0 \mathrm{E}-02$ & $U$ & $3.0 \mathrm{E}-02$ & $2.6 \mathrm{E}-02$ & $U$ & $2.6 \mathrm{E}-02$ & $7.0 \mathrm{E}-02$ & $U$ & $7.0 \mathrm{E}-02$ & $8.9 \mathrm{E}-02$ & $\mathrm{U}$ & $8.9 \mathrm{E}-02$ \\
\hline$B 8$ & J13D64 & $9 / 6 / 06$ & $3.1 \mathrm{E}-02$ & $\mathrm{U}$ & $4.8 \mathrm{E}-02$ & $2.7 \mathrm{E}-02$ & $\bar{U}$ & $2.7 \mathrm{E}-02$ & $3.2 \mathrm{E}-02$ & $U$ & $3.2 \mathrm{E}-02$ & $6.1 \mathrm{E}-02$ & $\bar{U}$ & $6.1 \mathrm{E}-02$ & $1.0 \mathrm{E}-01$ & $U$ & $1.0 \mathrm{E}-01$ \\
\hline $\mathrm{C9}$ & J13D65 & 9/6/06 & $4.0 \mathrm{E}-03$ & $U$ & $6.2 \mathrm{E}-02$ & $2.0 \mathrm{E}-02$ & $U$ & $2.0 \mathrm{E}-02$ & $2.0 \mathrm{E}-02$ & $U$ & 2.0E-02 & $4.5 \mathrm{E}-02$ & $\mathrm{U}$ & 4.5E-02 & $8.2 \mathrm{E}-02$ & $U$ & $8.2 \mathrm{E}-02$ \\
\hline $\mathrm{C} 10$ & J13D66 & $9 / 6 / 06$ & $8.18 \mathrm{E}-01$ & & $3.6 \mathrm{E}-02$ & $9.5 \mathrm{E}-02$ & & 2.4E-02 & $2.2 \mathrm{E}-02$ & $U$ & $2.2 \mathrm{E}-02$ & $5.6 \mathrm{E}-02$ & $U$ & $5.6 \mathrm{E}-02$ & 7.3E-02 & $\mathrm{U}$ & 7.3E-02 \\
\hline $\mathrm{C} 1$ & J13D67 & $9 / 6 / 06$ & $2.0 \mathrm{E}-02$ & $U$ & $4.4 \mathrm{E}-02$ & $4.0 \mathrm{E}-02$ & $\mathrm{U}$ & $4.0 \mathrm{E}-02$ & $3.2 \mathrm{E}-02$ & $\bar{U}$ & $3.2 \mathrm{E}-02$ & $9.1 \mathrm{E}-02$ & $U$ & $9.1 \mathrm{E}-02$ & $1.1 \mathrm{E}-01$ & $\mathrm{U}$ & $1.1 \mathrm{E}-01$ \\
\hline $\mathrm{C} 2$ & J13D68 & $9 / 6 / 06$ & $1.0 \mathrm{E}-02$ & $U$ & $6.5 \mathrm{E}-02$ & $5.5 \mathrm{E}-02$ & $\mathrm{U}$ & $5.5 \mathrm{E}-02$ & $5.4 \mathrm{E}-02$ & $U$ & $5.4 \mathrm{E}-02$ & $1.5 \mathrm{E}-01$ & $\bar{U}$ & $1.5 \mathrm{E}-01$ & $2.1 \mathrm{E}-01$ & $U$ & $2.1 \mathrm{E}-01$ \\
\hline D3 & J13D69 & 9/6/06 & $4.1 \mathrm{E}-02$ & $U$ & $6.6 \mathrm{E}-02$ & $3.5 \mathrm{E}-02$ & $\mathrm{U}$ & $3.5 \mathrm{E}-02$ & $3.8 \mathrm{E}-02$ & $\mathrm{U}$ & $3.8 \mathrm{E}-02$ & $8.2 \mathrm{E}-02$ & $U$ & $8.2 \mathrm{E}-02$ & $1.4 \mathrm{E}-01$ & $\mathrm{U}$ & $1.4 \mathrm{E}-01$ \\
\hline D4 & J13D70 & $9 / 6 / 06$ & $4.7 \mathrm{E}-02$ & $\mathrm{U}$ & $5.6 \mathrm{E}-02$ & $2.5 \mathrm{E}-02$ & $\mathrm{U}$ & $2.5 \mathrm{E}-02$ & $2.4 \mathrm{E}-02$ & $U$ & $2.4 \mathrm{E}-02$ & $6.5 \mathrm{E}-02$ & $U$ & $6.5 \mathrm{E}-02$ & 8.0E-02 & $\mathrm{U}$ & $8.0 \mathrm{E}-02$ \\
\hline $\mathrm{D} 5$ & J13D71 & $9 / 6 / 06$ & $6.1 \mathrm{E}-02$ & $U$ & $6.9 \mathrm{E}-02$ & $4.9 \mathrm{E}-02$ & $\mathrm{U}$ & $4.9 \mathrm{E}-02$ & $3.8 \mathrm{E}-02$ & $U$ & $3.8 \mathrm{E}-02$ & 1.1E-01 & $U$ & $1.1 \mathrm{E}-01$ & 1.3E-01 & $\mathrm{U}$ & $1.3 \mathrm{E}-01$ \\
\hline $\mathrm{D} 6$ & $\begin{array}{l}J 13 D 72 \\
\end{array}$ & $9 / 6 / 06$ & $-2.0 \mathrm{E}-02$ & $U$ & $5.8 \mathrm{E}-02$ & $4.9 \mathrm{E}-02$ & $U$ & $4.9 \mathrm{E}-02$ & $5.1 \mathrm{E}-02$ & $U$ & $5.1 \mathrm{E}-02$ & $1.4 \mathrm{E}-01$ & $\mathrm{U}$ & $1.4 \mathrm{E}-01$ & $1.8 \mathrm{E}-01$ & $\bar{U}$ & $1.8 \mathrm{E}-01$ \\
\hline $\mathrm{A} 1$ & J13D73 & $9 / 6 / 06$ & $8.0 \mathrm{E}-03$ & $\mathrm{U}$ & $4.9 \mathrm{E}-02$ & $4.2 E-02$ & $U$ & $4.2 \mathrm{E}-02$ & $4.0 \mathrm{E}-02$ & $U$ & $4.0 \mathrm{E}-02$ & 1.0E-01 & $\mathrm{U}$ & $1.0 \mathrm{E}-01$ & 1.3E-01 & $\mathrm{U}$ & $1.3 \mathrm{E}-01$ \\
\hline $\mathrm{A} 3$ & J13D75 & $9 / 6 / 06$ & 0 & $U$ & $2.1 \mathrm{E}-01$ & $2.6 \mathrm{E}-02$ & $U$ & $2.6 \mathrm{E}-02$ & $2.5 \mathrm{E}-02$ & $\mathrm{U}$ & $2.5 \mathrm{E}-02$ & $7.4 \mathrm{E}-02$ & $U$ & 7.4E-02 & 8.6E-02 & $U$ & $8.6 \mathrm{E}-02$ \\
\hline A4 & J13D62 & $9 / 6 / 06$ & $1.1 \mathrm{E}-02$ & $U$ & $6.0 \mathrm{E}-02$ & $4.5 \mathrm{E}-02$ & $U$ & $4.5 \mathrm{E}-02$ & $4.6 \mathrm{E}-02$ & $\mathrm{U}$ & $4.6 \mathrm{E}-02$ & $1.2 \mathrm{E}-01$ & $U$ & $1.2 \mathrm{E}-01$ & 1.7E-01 & $\mathrm{U}$ & $1.7 \mathrm{E}-01$ \\
\hline $\mathrm{A} 2$ & J13D74 & $9 / 6 / 06$ & $-1.5 \mathrm{E}-02$ & $U$ & $5.5 \mathrm{E}-02$ & $3.1 \mathrm{E}-02$ & $U$ & $3.1 \mathrm{E}-02$ & $2.7 \mathrm{E}-02$ & $\mathrm{U}$ & $2.7 \mathrm{E}-02$ & $7.7 \mathrm{E}-02$ & $\mathrm{U}$ & 7.7E-02 & $8.2 E-02$ & $\bar{U}$ & $8.2 \mathrm{E}-02$ \\
\hline $\begin{array}{l}\text { Duplicate } \\
\text { of } J 13 D 74\end{array}$ & J13D59 & 9/6/06 & 2.2E-02 & $u$ & $6.9 \mathrm{E}-02$ & $E-02$ & $U$ & E-02 & E-02 & $U$ & $E-02$ & $E-01$ & U & 1.1E-01 & 1.5E-01 & U & $1.5 \mathrm{E}-01$ \\
\hline $\begin{array}{c}\text { Split of } \\
\mathrm{J} 13 \mathrm{D} 74 \\
\end{array}$ & J13D76 & 9/6/06 & $6.77 \mathrm{E}-03$ & $u$ & 4.06E-02 & 6E-03 & $U$ & 02 & $60 \mathrm{E}-05$ & U & 1.55E-02 & -03 & $u$ & 3.24E-02 & $E-02$ & $\mathrm{U}$ & $=-02$ \\
\hline
\end{tabular}


Table B-4. 618-2, 618-3, 618-8 Staging Pile/Decon Pad Cleanup Verification Data. (5 pages)

\begin{tabular}{|c|c|c|c|c|c|c|c|c|c|c|c|c|c|c|c|c|c|}
\hline \multirow{2}{*}{$\begin{array}{c}\text { Sampling } \\
\text { Area }\end{array}$} & \multirow{2}{*}{$\begin{array}{l}\text { Sample } \\
\text { Number }\end{array}$} & \multirow{2}{*}{\begin{tabular}{|c|} 
Sample \\
Date
\end{tabular}} & \multicolumn{3}{|c|}{ Europium-155 } & \multicolumn{3}{|c|}{ Nickel-63 } & \multicolumn{3}{|c|}{ Tritium } & \multicolumn{3}{|c|}{ Plutonium-238 } & \multicolumn{3}{|c|}{ Plutonium-239/240 } \\
\hline & & & $\mathrm{pCi} / \mathrm{g}$ & Q & MDA & $\mathrm{pCi} / \mathrm{g}$ & Q & MDA & $\mathrm{pCi} / \mathrm{g}$ & $\mathbf{Q}$ & MDA & $\mathrm{pCi} / \mathrm{g}$ & $Q$ & MDA & $\mathrm{pCi} / \mathrm{g}$ & Q & MDA \\
\hline B5 & J13D61 & $9 / 6 / 06$ & 1.0E-01 & $u$ & $1.0 \mathrm{E}-01$ & 3.56E-01 & $\mathrm{U}$ & $3.6 \mathrm{E}+00$ & $8.5 \mathrm{E}-02$ & UJ & $2.4 \mathrm{E}+00$ & $-1.06 E-01$ & UJ & 8.1E-01 & 0 & UJ & $8.1 \mathrm{E}-01$ \\
\hline $\mathrm{B} 6$ & J13D60 & $9 / 6 / 06$ & $1.0 \mathrm{E}-01$ & $\mathrm{U}$ & $1.0 \mathrm{E}-01$ & $2.78 \mathrm{E}-01$ & $\mathrm{U}$ & $3.7 \mathrm{E}+00$ & $2.22 \mathrm{E}-01$ & UJ & $2.5 \mathrm{E}+00$ & $\overline{0}$ & $\mathrm{U}$ & $4.0 \mathrm{E}-01$ & 0 & $\mathrm{U}$ & $4.0 \mathrm{E}-01$ \\
\hline B7 & J13D63 & $9 / 6 / 06$ & $6.3 \mathrm{E}-02$ & $\mathrm{Uu}$ & $6.3 \mathrm{E}-02$ & $1.23 \mathrm{E}+00$ & $\mathrm{Uu}$ & $3.8 \mathrm{E}+00$ & $8.61 \mathrm{E}-01$ & UJ & $2.7 \mathrm{E}+00$ & $-5.2 \mathrm{E}-02$ & $\mathrm{U}$ & $4.0 \mathrm{E}-01$ & $5.2 \mathrm{E}-02$ & $u$ & $4.0 \mathrm{E}-01$ \\
\hline$B 8$ & J13D64 & $9 / 6 / 06$ & $8.4 \mathrm{E}-02$ & $\mathrm{U}$ & $8.4 \mathrm{E}-02$ & $1.02 \mathrm{E}+00$ & $\mathrm{U}$ & $3.7 \mathrm{E}+00$ & $-1.28 \mathrm{E}-01$ & UJ & $2.4 \mathrm{E}+00$ & $5.2 \mathrm{E}-02$ & $\bar{U}$ & $4.0 \mathrm{E}-01$ & $1.56 \mathrm{E}-01$ & $u$ & $4.0 \mathrm{E}-01$ \\
\hline $\mathrm{C9}$ & J13D65 & $9 / 6 / 06$ & $4.8 \mathrm{E}-02$ & $\mathrm{U}$ & $4.8 \mathrm{E}-02$ & $5.47 \mathrm{E}-01$ & $\mathrm{U}$ & $3.7 \mathrm{E}+00$ & 0 & UJ & $2.3 \mathrm{E}+00$ & 0 & $\mathrm{U}$ & $4.0 \mathrm{E}-01$ & $6.26 \mathrm{E}-01$ & & $4.0 \mathrm{E}-01$ \\
\hline $\mathrm{C} 10$ & J13D66 & $9 / 6 / 06$ & $7.8 \mathrm{E}-02$ & $\mathrm{U}$ & $7.8 \mathrm{E}-02$ & 0 & $\mathrm{U}$ & $3.6 \mathrm{E}+00$ & $2.33 \mathrm{E}+00$ & J & $2.3 E+00$ & $1.03 \mathrm{E}-01$ & $\mathrm{U}$ & $3.9 \mathrm{E}-01$ & $1.00 \mathrm{E}+01$ & & $3.9 \mathrm{E}-01$ \\
\hline $\mathrm{C} 1$ & J13D67 & $9 / 6 / 06$ & $8.1 \mathrm{E}-02$ & $\mathrm{U}$ & $8.1 \mathrm{E}-02$ & $1.31 \mathrm{E}-01$ & $\mathrm{U}$ & $3.5 \mathrm{E}+00$ & $1.13 \mathrm{E}+00$ & UJ & $2.5 \mathrm{E}+00$ & $4.6 \mathrm{E}-02$ & $\mathrm{U}$ & $4.4 \mathrm{E}-01$ & $1.86 \mathrm{E}-01$ & $\mathrm{U}$ & $3.5 \mathrm{E}-01$ \\
\hline $\mathrm{C} 2$ & J13D68 & $9 / 6 / 06$ & $2.1 \mathrm{E}-01$ & $\mathrm{U}$ & $2.1 \mathrm{E}-01$ & $6.74 \mathrm{E}-01$ & $U$ & $3.6 E+00$ & $6.95 \mathrm{E}-01$ & \begin{tabular}{|l|} 
UJ \\
\end{tabular} & $2.3 E+00$ & $2.48 \mathrm{E}-01$ & $\bar{U}$ & $4.0 \mathrm{E}-01$ & $4.1 \mathrm{E}-02$ & $\mathrm{U}$ & $3.2 \mathrm{E}-01$ \\
\hline D3 & J13D69 & $9 / 6 / 06$ & $1.2 \mathrm{E}-01$ & $\mathrm{U}$ & $1.2 \mathrm{E}-01$ & $1.22 \mathrm{E}+00$ & $\mathrm{U}$ & $3.7 \mathrm{E}+00$ & $1.29 \mathrm{E}+00$ & UJ & $2.5 \mathrm{E}+00$ & $-9.7 \mathrm{E}-02$ & $\bar{U}$ & $4.7 \mathrm{E}-01$ & $4.9 \mathrm{E}-02$ & $U$ & $3.7 \mathrm{E}-01$ \\
\hline D4 & J13D70 & $9 / 6 / 06$ & $9.4 \mathrm{E}-02$ & U & $9.4 \mathrm{E}-02$ & $6.9 \mathrm{E}-01$ & $\mathrm{U}$ & $3.5 \mathrm{E}+00$ & $2.51 \mathrm{E}-01$ & UJ & $2.3 E+00$ & 0 & $\mathrm{U}$ & $4.0 \mathrm{E}-01$ & $5.2 \mathrm{E}-02$ & $\mathrm{U}$ & $4.0 \mathrm{E}-01$ \\
\hline D5 & J13D71 & 9/6/06 & $1.0 \mathrm{E}-01$ & $U$ & $1.0 \mathrm{E}-01$ & $1.49 \mathrm{E}+00$ & $\mathrm{U}$ & $3.5 \mathrm{E}+00$ & $6.8 \mathrm{E}-02$ & UJ & $2.5 \mathrm{E}+00$ & 0 & $\mathrm{U}$ & 4.2E-01 & $1.05 \mathrm{E}+00$ & & $4.2 \mathrm{E}-01$ \\
\hline $\mathrm{D} 6$ & J13D72 & $9 / 6 / 06$ & $1.9 \mathrm{E}-01$ & $\mathrm{U}$ & $1.9 \mathrm{E}-01$ & $7.79 \mathrm{E}-01$ & $\mathrm{U}$ & $3.5 \mathrm{E}+00$ & $1.14 \mathrm{E}+00$ & UJ & $2.5 \mathrm{E}+00$ & $-6.2 \mathrm{E}-02$ & $\mathrm{U}$ & 4.7E-01 & $1.23 \mathrm{E}-01$ & $U$ & $4.7 \mathrm{E}-01$ \\
\hline $\mathrm{A} 1$ & J13D73 & $9 / 6 / 06$ & $9.0 \mathrm{E}-02$ & $\mathrm{U}$ & $9.0 \mathrm{E}-02$ & $2.99 \mathrm{E}-01$ & $\mathrm{U}$ & $3.4 \mathrm{E}+00$ & $4.13 \mathrm{E}-01$ & UJ & $2.3 E+00$ & $6.1 \mathrm{E}-02$ & $U$ & $4.7 \mathrm{E}-01$ & 0 & $\mathrm{U}$ & $4.7 \mathrm{E}-01$ \\
\hline $\mathrm{A3}$ & J13D75 & $9 / 6 / 06$ & $1.1 \mathrm{E}-01$ & $\mathrm{U}$ & $1.1 \mathrm{E}-01$ & $7.6 \mathrm{E}-01$ & $U$ & $3.6 \mathrm{E}+00$ & 7.77E-01 & UJ & $2.3 E+00$ & \begin{tabular}{|c|}
$1.52 \mathrm{E}-01$ \\
\end{tabular} & $\mathrm{U}$ & $5.8 \mathrm{E}-01$ & 0 & $\mathrm{U}$ & $5.8 \mathrm{E}-01$ \\
\hline A4 & J13D62 & $9 / 6 / 06$ & $1.8 \mathrm{E}-01$ & $U$ & $1.8 \mathrm{E}-01$ & $9.08 \mathrm{E}-01$ & $\mathrm{U}$ & $4.1 \mathrm{E}+00$ & $1.12 \mathrm{E}+00$ & UJ & $2.4 \mathrm{E}+00$ & 1.32E-01 & $U$ & $5.0 \mathrm{E}-01$ & 0 & $U$ & $5.0 \mathrm{E}-01$ \\
\hline $\mathrm{A} 2$ & J13D74 & $9 / 6 / 06$ & 6.6E-02 & U & 6.6E-02 & $5.03 \mathrm{E}-01$ & $\mathrm{U}$ & $3.7 \mathrm{E}+00$ & $1.18 \mathrm{E}+00$ & UJ & $2.4 \mathrm{E}+00$ & $-6.2 \mathrm{E}-02$ & $u$ & 4.7E-01 & 0 & $\mathrm{U}$ & 4.7E-01 \\
\hline $\begin{array}{c}\text { Duplicate of } \\
\text { J13D74 }\end{array}$ & J13D59 & 9/6/06 & $9.9 \mathrm{E}-02$ & $u$ & $9.9 \mathrm{E}-02$ & 2.15E-01 & $u$ & $4.4 \mathrm{E}+00$ & $1.11 \mathrm{E}+00$ & UJ & $2.3 E+00$ & 0 & u & $4.8 \mathrm{E}-01$ & 0 & $u$ & $4.8 \mathrm{E}-01$ \\
\hline $\begin{array}{c}\text { Split of } \\
\text { J13D74 }\end{array}$ & J13D76 & 9/6/06 & $4.61 \mathrm{E}-02$ & $u$ & $4.64 \mathrm{E}-02$ & $4.56 \mathrm{E}+00$ & $U$ & $6.14 \mathrm{E}+00$ & $-7.58 \mathrm{E}-04$ & U & 3.67E-02 & 0 & $U$ & $6.46 \mathrm{E}-02$ & E-02 & $u$ & $1.35 \mathrm{E}-01$ \\
\hline
\end{tabular}


Table B-4. 618-2, 618-3, 618-8 Staging Pile/Decon Pad Cleanup Verification Data. (5 pages)

\begin{tabular}{|c|c|c|c|c|c|c|c|c|c|c|c|c|c|c|c|c|}
\hline \multirow{2}{*}{$\begin{array}{c}\text { Sampling } \\
\text { Area }\end{array}$} & \multirow{2}{*}{$\begin{array}{l}\text { Sample } \\
\text { Number }\end{array}$} & \multirow{2}{*}{$\begin{array}{c}\text { Sample } \\
\text { Date }\end{array}$} & \multicolumn{3}{|c|}{ Plutonium-241 } & \multicolumn{3}{|c|}{ Strontium-90 } & \multicolumn{3}{|c|}{ Uranium-233/234 } & \multicolumn{3}{|c|}{ Uranium-235 } & \multicolumn{2}{|c|}{ Uranium-238 } \\
\hline & & & $\mathrm{pCi} / \mathrm{g}$ & Q & MDA & $\mathrm{pCi} / \mathrm{g}$ & Q & MDA & $\mathrm{pCi} / \mathrm{g}$ & $\mathbf{Q}$ & MDA & $\mathrm{pCi} / \mathrm{g}$ & Q & MDA & $\mathrm{pCi} / \mathrm{g}$ & MDA \\
\hline$\overline{B 5}$ & J13D61 & $9 / 6 / 06$ & $2.92 \mathrm{E}+00$ & $u$ & $2.2 E+01$ & $2.8 \mathrm{E}-02$ & U & $2.7 E-01$ & $5.51 \mathrm{E}-01$ & & $1.6 \mathrm{E}-01$ & 0 & $\bar{U}$ & $1.9 \mathrm{E}-01$ & $4.69 \mathrm{E}-01$ & $1.6 \mathrm{E}-01$ \\
\hline $\mathrm{B} 6$ & J13D60 & $9 / 6 / 06$ & $-5.17 \mathrm{E}+00$ & $\mathrm{U}$ & $2.2 \mathrm{E}+01$ & $-2.9 \mathrm{E}-02$ & $\mathrm{U}$ & $2.4 \mathrm{E}-01$ & $1.68 \mathrm{E}+00$ & & $1.5 \mathrm{E}-01$ & $1.96 \mathrm{E}-01$ & & $1.9 \mathrm{E}-01$ & $1.76 \mathrm{E}+00$ & $1.5 \mathrm{E}-01$ \\
\hline$\overline{B 7}$ & J13D63 & 9/6/06 & $-1.14 \mathrm{E}+00$ & $U$ & $2.4 \mathrm{E}+01$ & $4.0 \mathrm{E}-02$ & $U$ & $2.2 E-01$ & $6.80 \mathrm{E}-01$ & & $2.3 \mathrm{E}-01$ & $7.2 \mathrm{E}-02$ & $\mathrm{U}$ & $2.7 \mathrm{E}-01$ & 7.69E-01 & $2.3 \mathrm{E}-01$ \\
\hline B8 & J13D64 & 9/6/06 & 0 & $u$ & $2.3 E+01$ & $1.10 \mathrm{E}-01$ & $\mathrm{U}$ & 2.3E-01 & 5.57E-01 & & 1.7E-01 & 2.7E-02 & U & $2.1 E-01$ & $7.80 \mathrm{E}-01$ & 1.7E-01 \\
\hline C9 & J13D65 & 9/6/06 & $-1.90 \mathrm{E}-01$ & $u$ & $2.4 \mathrm{E}+01$ & $1.25 \mathrm{E}-01$ & $U$ & $2.4 \mathrm{E}-01$ & $1.20 \mathrm{E}+00$ & & 4.3E-02 & $1.34 \mathrm{E}-01$ & & $2.4 \mathrm{E}-02$ & $1.19 \mathrm{E}+00$ & $3.2 \mathrm{E}-02$ \\
\hline $\mathrm{C} 10$ & J13D66 & $9 / 6 / 06$ & $-1.79 E-01$ & U & $2.3 E+01$ & $-8.6 \mathrm{E}-02$ & 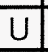 & $2.6 \mathrm{E}-01$ & $1.75 \mathrm{E}+00$ & & $4.2 E-02$ & 1.39E-01 & & $2.5 \mathrm{E}-02$ & $1.83 \mathrm{E}+00$ & $3.7 \mathrm{E}-02$ \\
\hline $\mathrm{C} 1$ & J13D67 & 9/6/06 & $3.22 E+00$ & U & $2.4 \mathrm{E}+01$ & $1.38 \mathrm{E}-01$ & U & $3.0 E-01$ & $7.50 \mathrm{E}-01$ & & $3.2 E-02$ & $5.6 \mathrm{E}-02$ & & $3.9 E-02$ & $7.21 \mathrm{E}-01$ & $3.5 \mathrm{E}-02$ \\
\hline $\mathrm{C} 2$ & J13D68 & 9/6/06 & $-2.04 E-01$ & U & $2.6 \mathrm{E}+01$ & $-4.5 E-02$ & 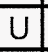 & $2.4 \mathrm{E}-01$ & $8.36 \mathrm{E}-01$ & & $4.0 \mathrm{E}-01$ & $8.4 \mathrm{E}-02$ & & $2.8 \mathrm{E}-02$ & $8.02 E-01$ & $3.3 \mathrm{E}-02$ \\
\hline D3 & J13D69 & $9 / 6 / 06$ & $2.36 \mathrm{E}+00$ & U & $2.5 E+01$ & $-5.0 \mathrm{E}-03$ & $U$ & $2.4 \mathrm{E}-01$ & $7.26 \mathrm{E}-01$ & & $4.0 \mathrm{E}-01$ & $8.3 E-02$ & & $2.5 E-02$ & $6.13 E-01$ & $3.7 \mathrm{E}-02$ \\
\hline$\overline{D 4}$ & J13D70 & $9 / 6 / 06$ & $-1.94 E+00$ & U & $2.2 E+01$ & $1.46 \mathrm{E}-01$ & $U$ & 3.0E-01 & $1.04 \mathrm{E}+00$ & & $5.5 \mathrm{E}-02$ & $8.6 \mathrm{E}-02$ & & $4.1 \mathrm{E}-02$ & $9.77 \mathrm{E}-01$ & $5.5 \mathrm{E}-02$ \\
\hline D5 & J13D71 & 9/6/06 & $9.22 \mathrm{E}+00$ & u & $2.1 E+01$ & $-1.1 \mathrm{E}-02$ & $U$ & $2.1 \mathrm{E}-01$ & $4.07 \mathrm{E}+00$ & & $5.3 \mathrm{E}-02$ & $2.67 \mathrm{E}-01$ & & $2.9 \mathrm{E}-02$ & $3.82 \mathrm{E}+00$ & $5.0 \mathrm{E}-02$ \\
\hline D6 & J13D72 & $9 / 6 / 06$ & $2.62 \mathrm{E}+00$ & U & $2.5 \mathrm{E}+01$ & $5.7 \mathrm{E}-02$ & $\mathrm{U}$ & $2.7 \mathrm{E}-01$ & $6.17 \mathrm{E}-01$ & & $3.1 \mathrm{E}-02$ & $5.1 \mathrm{E}-02$ & & $2.6 E-02$ & $6.70 \mathrm{E}-01$ & $2.7 \mathrm{E}-02$ \\
\hline $\mathrm{A} 1$ & J13D73 & $9 / 6 / 06$ & $1.81 \mathrm{E}+00$ & $\mathrm{U}$ & $2.3 E+01$ & $-6.1 \mathrm{E}-02$ & $\mathrm{U}$ & $3.4 \mathrm{E}-01$ & $8.38 \mathrm{E}-01$ & & $1.8 \mathrm{E}-01$ & $8.5 \mathrm{E}-02$ & $U$ & $2.2 E-01$ & 5.82E-01 & $1.8 \mathrm{E}-01$ \\
\hline $\mathrm{A} 3$ & J13D75 & 9/6/06 & $3.55 \mathrm{E}+00$ & $u$ & $2.8 \mathrm{E}+01$ & $2.0 \mathrm{E}-03$ & $\bar{U}$ & $2.1 \mathrm{E}-01$ & $4.69 \mathrm{E}-01$ & & $1.9 \mathrm{E}-01$ & $9.0 \mathrm{E}-02$ & $U$ & $2.3 \mathrm{E}-01$ & $4.69 \mathrm{E}-01$ & $1.9 \mathrm{E}-01$ \\
\hline A4 & J13D62 & $9 / 6 / 06$ & $1.73 \mathrm{E}+00$ & $U$ & $2.7 \mathrm{E}+01$ & $4.3 \mathrm{E}-02$ & $\mathrm{U}$ & $2.7 \mathrm{E}-01$ & $6.43 E-01$ & & $1.5 \mathrm{E}-01$ & $2.4 \mathrm{E}-02$ & $U$ & $1.9 \mathrm{E}-01$ & $5.62 \mathrm{E}-01$ & $1.5 \mathrm{E}-01$ \\
\hline $\mathrm{A} 2$ & J13D74 & $\begin{array}{l}9 / 6 / 06 \\
\end{array}$ & $7.42 \mathrm{E}+00$ & $u$ & $2.7 E+01$ & $-2.3 E-02$ & U & $2.1 \mathrm{E}-01$ & $7.45 \mathrm{E}-01$ & & $1.9 \mathrm{E}-01$ & 0 & $U$ & $2.3 \mathrm{E}-01$ & $8.45 \mathrm{E}-01$ & $1.9 \mathrm{E}-01$ \\
\hline $\begin{array}{c}\text { Duplicate } \\
\text { of } J 13 D 74 \\
\end{array}$ & J13D59 & 9/6/06 & $-4.44 E+00$ & $u$ & $2.4 \mathrm{E}+01$ & $-1.05 E-01$ & $u$ & 2.7E-01 & $E-01$ & & -01 & $3.5 \mathrm{E}-02$ & $u$ & $E-01$ & $30 \mathrm{E}-01$ & 2.2E-01 \\
\hline $\begin{array}{l}\text { Split of } \\
\text { J13D74 }\end{array}$ & 6 & 6 & -4 & $u$ & -00 & $1.35 \mathrm{E}-02$ & $u$ & 1.12E-01 & $1.72 \mathrm{E}+00$ & & 2.95E-02 & 7.87E-02 & & $3.65 \mathrm{E}-02$ & $1.75 \mathrm{E}+00$ & $3.65 \mathrm{E}-02$ \\
\hline
\end{tabular}


Table B-5. 618-2 Verification Sample Results (Biased Samples). (3 pages)

\begin{tabular}{|c|c|c|c|c|c|c|c|c|c|c|c|c|c|c|c|c|c|}
\hline \multirow{2}{*}{ Description } & \multirow{2}{*}{\begin{tabular}{|c|} 
Sample \\
Number
\end{tabular}} & \multirow{2}{*}{$\begin{array}{c}\text { Sample } \\
\text { Date }\end{array}$} & \multicolumn{3}{|c|}{ Arsenic } & \multicolumn{3}{|c|}{ Barium } & \multicolumn{3}{|c|}{ Cadmium } & \multicolumn{3}{|c|}{ Chromium } & \multicolumn{3}{|c|}{ Lead } \\
\hline & & & $\mathrm{mg} / \mathrm{kg}$ & $Q$ & PQL & $\mathrm{mg} / \mathrm{kg}$ & $Q$ & $P Q L$ & $\mathrm{mg} / \mathrm{kg}$ & $\mathbf{Q}$ & \begin{tabular}{|l|}
$P Q L$ \\
\end{tabular} & $\mathrm{mg} / \mathrm{kg}$ & $\mathbf{Q}$ & $\mathrm{PQL}$ & $\mathrm{mg} / \mathrm{kg}$ & Q & PQL \\
\hline Discolo & J13H86 & 09/28/06 & 1.4 & & 1.1 & 68 & & 0.06 & 0.09 & $U$ & 0.09 & 3.8 & & 0.21 & 1.6 & & 0.37 \\
\hline Discolored soil & J13H87 & \begin{tabular}{|l|}
$09 / 28 / 06$ \\
\end{tabular} & 1.7 & & 1.1 & 64.3 & & 0.06 & 0.16 & & 0.09 & 3.2 & & 0.21 & 2.3 & & 0.36 \\
\hline Hot spot & NO & 09/26/06 & 1.6 & & 1.1 & 107 & & 0.06 & 0.09 & $\mathrm{U}$ & 0.09 & 4.4 & & 4.4 & 2.4 & & 0.35 \\
\hline Hot spot & $\mathrm{N} 1$ & $\mid 09 / 26 / 06$ & 1.7 & & 1.1 & 58.8 & & 0.06 & 0.20 & & 0.09 & 4.4 & & 0.20 & 3.3 & & 0.34 \\
\hline Hot sp & $\mathrm{N} 2$ & $\mid 09 / 26 / 06$ & 1.8 & & 1.1 & 109 & & 0.06 & 0.09 & $\mathrm{U}$ & 0.09 & 10.4 & & 0.21 & 6.6 & & 0.35 \\
\hline Hot si & N3 & $09 / 26 / 06$ & 1.7 & & 1.1 & 56.1 & & 0.06 & 0.09 & $\mathrm{U}$ & 0.09 & 4.0 & & 0.20 & 1.7 & & 0.35 \\
\hline Hot S & N4 & $09 / 26 / 06$ & 1.6 & & 1.1 & 60.2 & & 0.06 & 0.09 & $\mathrm{U}$ & 0.09 & 3.9 & & 0.20 & 1.8 & & 0.35 \\
\hline Beneath s & $\mathrm{J} 5$ & \begin{tabular}{|l|}
$09 / 07 / 06$ \\
\end{tabular} & 2.5 & & 1.1 & 101 & & 0.06 & 0.09 & $\mathrm{U}$ & 0.09 & 6.9 & & 0.20 & 4.5 & & 0.35 \\
\hline Beneath oil drums & J13DH9 & \begin{tabular}{|l|}
$09 / 07 / 06$ \\
\end{tabular} & 3.3 & & 1.1 & 84.5 & & 0.06 & 0.09 & $\mathrm{U}$ & 0.09 & 7.9 & & 0.21 & 6.8 & & 0.35 \\
\hline
\end{tabular}

\begin{tabular}{|c|c|c|c|c|c|c|c|c|c|c|c|c|c|c|}
\hline \multirow{2}{*}{ Description } & \multirow{2}{*}{\begin{tabular}{|l|} 
Sample \\
Number
\end{tabular}} & \multirow{2}{*}{$\begin{array}{c}\text { Sample } \\
\text { Date }\end{array}$} & \multicolumn{3}{|c|}{ Selenium } & \multicolumn{3}{|c|}{ Silver } & \multicolumn{3}{|c|}{ Tin } & \multicolumn{3}{|c|}{ Uranium } \\
\hline & & & $\mathrm{mg} / \mathrm{kg}$ & Q & $P Q L$ & $\mathrm{mg} / \mathrm{kg}$ & $\mathbf{Q}$ & $\overline{P Q L}$ & $\mathrm{mg} / \mathrm{kg}$ & $\mathbf{Q}$ & PQL & $\mathrm{mg} / \mathrm{kg}$ & $\mathbf{Q}$ & PQL \\
\hline Discolored soil & $\mathrm{J} 13 \mathrm{H} 86$ & $09 / 28 / 06$ & 1.2 & & 0.80 & 0.34 & $U$ & 0.34 & 1.1 & $U$ & 1.1 & 16.5 & & 0.10 \\
\hline Discolored soil & $\mathrm{J} 13 \mathrm{H} 87$ & $09 / 28 / 06$ & 0.78 & $U$ & 0.78 & 0.33 & $\mathrm{U}$ & 0.33 & 1.0 & $U$ & 1.0 & 20.1 & & 0.10 \\
\hline Hot spot & J13DNO & $09 / 26 / 06$ & 0.76 & $\mathrm{U}$ & 0.76 & 0.32 & $\mathrm{U}$ & 0.32 & 1.0 & $U$ & 1.0 & 148 & & 0.98 \\
\hline Hot spot & J13DN1 & $09 / 26 / 06$ & 0.74 & U & 0.74 & 0.31 & $U$ & 0.31 & 1.0 & $U$ & 1.0 & 26.0 & & 0.098 \\
\hline Hot spot & 3DN2 & $09 / 26 / 06$ & 0.76 & $\mathrm{U}$ & 0.76 & 0.32 & $U$ & 0.32 & 42.5 & & 1.0 & 32.8 & & 0.098 \\
\hline Hot spot & J13DN3 & $09 / 26 / 06$ & 0.75 & $U$ & 0.75 & 0.32 & & 0.32 & 1.0 & $\mathrm{U}$ & 1.0 & 0.878 & & 0.010 \\
\hline Hot spot & J13DN4 & $09 / 26 / 06$ & 0.94 & & 0.76 & 0.32 & $\bar{U}$ & 0.32 & 1.2 & & 1.0 & 0.952 & & 0.010 \\
\hline Beneath safe & J13DJ5 & $09 / 07 / 06$ & 0.82 & & 0.76 & 0.32 & $\bar{U}$ & 0.32 & 1.6 & $J$ & 1.0 & 5.80 & & 0.007 \\
\hline Beneath oil drums & $\mathrm{J13 \textrm {DH } 9}$ & $09 / 07 / 06$ & 0.77 & $U$ & 0.77 & 0.6 & & 0.32 & 1.9 & $\mathrm{~J}$ & 1.0 & 29.1 & & 0.072 \\
\hline
\end{tabular}

\begin{tabular}{|c|c|c|c|c|c|c|c|c|c|c|c|c|c|c|c|c|c|c|c|c|}
\hline \multirow[b]{2}{*}{ Description } & \multirow{2}{*}{$\begin{array}{l}\text { Sample } \\
\text { Number }\end{array}$} & \multirow[b]{2}{*}{$\begin{array}{c}\text { Sample } \\
\text { Date }\end{array}$} & \multicolumn{3}{|c|}{ Aroclor-1016 } & \multicolumn{3}{|c|}{ Aroclor-1221 } & \multicolumn{3}{|c|}{ Aroclor-1232 } & \multicolumn{3}{|c|}{ Aroclor-1242 } & \multicolumn{3}{|c|}{ Aroclor-1248 } & \multicolumn{3}{|c|}{ Aroclor-1254 } \\
\hline & & & ug/ $/ \mathbf{k g}$ & $\mathbf{Q}$ & PQL & $\mathrm{ug} / \mathrm{kg}$ & $\mathbf{Q}$ & PQL & $\mathrm{kg}$ & $\mathbf{Q}$ & $P Q L$ & $\mathrm{gg}$ & $\mathbf{Q}$ & $P Q L$ & $\mathrm{gg}$ & $\mathbf{Q}$ & PQL & $\begin{array}{c}\text { ug/k } \\
\mathrm{g}\end{array}$ & $\mathbf{Q}$ & PQL \\
\hline Beneath oil drums & J13DH9 & \begin{tabular}{|l|}
$09 / 07 / 06$ \\
\end{tabular} & 14 & U & 14 & 14 & U & 14 & 14 & $\bar{U}$ & 14 & 14 & $\mathrm{U}$ & 14 & 14 & U & 14 & 6.9 & $\mathrm{~J}$ & 14 \\
\hline
\end{tabular}

\begin{tabular}{|c|c|c|c|c|c|c|c|c|}
\hline \multirow{2}{*}{ Description } & Sample & Sample & \multicolumn{3}{|c|}{ Aroclor-1260 } & \multicolumn{3}{|c|}{ TPH } \\
\cline { 4 - 8 } & Number & Date & ug/kg & Q & PQL & $\mathrm{mg} / \mathrm{kg}$ & $\mathrm{Q}$ & $\mathrm{PQL}$ \\
\hline Beneath oil drums & J13DH9 & $09 / 07 / 06$ & 14 & $\mathrm{U}$ & 14 & 145 & $\mathrm{U}$ & 145 \\
\hline
\end{tabular}


Table B-5. 618-2 Verification Sample Results (Biased Samples). (3 pages)

\begin{tabular}{|c|c|c|c|c|c|c|c|c|c|c|c|c|c|c|c|c|c|}
\hline \multirow{2}{*}{ Description } & \multirow{2}{*}{$\begin{array}{l}\text { Sample } \\
\text { Number }\end{array}$} & \multirow{2}{*}{$\begin{array}{c}\text { Sample } \\
\text { Date }\end{array}$} & \multicolumn{3}{|c|}{ Americium-241 } & \multicolumn{3}{|c|}{ Cesium-137 } & \multicolumn{3}{|c|}{ Cobalt-60 } & \multicolumn{3}{|c|}{ Europium-152 } & \multicolumn{3}{|c|}{ Europium-154 } \\
\hline & & & $\mathrm{pCi} / \mathrm{g}$ & $\mathbf{Q}$ & MDA & $\mathrm{pCi} / \mathrm{g}$ & $\mathbf{Q}$ & MDA & $\mathrm{pCi} / \mathrm{g}$ & $\mathbf{Q}$ & MDA & $\mathrm{pCi} / \mathrm{g}$ & $\mathbf{Q}$ & MDA & $\mathrm{pCi} / \mathrm{g}$ & $\mathbf{Q}$ & MDA \\
\hline Discolored soil & J13H86 & $09 / 28 / 06$ & 0.086 & $\bar{U}$ & 0.16 & 0.018 & $\bar{U}$ & 0.018 & 0.053 & $u$ & 0.053 & 0.051 & $\mathrm{U}$ & 0.051 & 0.065 & $\mathrm{U}$ & 0.065 \\
\hline Disc & & $09 / 2$ & 0.842 & & 0.18 & 39 & $\mathrm{U}$ & 0.039 & 0.046 & $u$ & 0.046 & 0.11 & $u$ & 0.11 & 0.14 & $U$ & 0.14 \\
\hline Hot spot & No & $09 / 26 / 06$ & 0.044 & & 0.041 & 0.249 & & 0.056 & 0.047 & $U$ & 0.047 & 0.15 & $U$ & 0.15 & 0.16 & $\mathrm{U}$ & 0.16 \\
\hline Hot spot & J13DN1 & $09 / 26 / 06$ & 6.47 & & 0.047 & 0.158 & & 0.028 & 0.025 & $U$ & 0.025 & 0.066 & $U$ & 0.066 & 0.084 & $\mathrm{U}$ & 0.084 \\
\hline Hot spot & J13DN2 & $09 / 26 / 06$ & 9.19 & & 0.046 & 1.12 & & 0.048 & 0.033 & $U$ & 0.033 & 0.098 & $U$ & 0.098 & 0.1 & $\mathrm{U}$ & 0.1 \\
\hline Hot spot & J13DN3 & $09 / 26 / 06$ & -0.003 & $\mathrm{U}$ & 0.045 & 0.126 & & 0.027 & 0.037 & $U$ & 0.037 & 0.063 & $U$ & 0.063 & 0.077 & $\mathrm{U}$ & 0.077 \\
\hline Hot spot & J13DN4 & $09 / 26 / 06$ & 0 & $U$ & 0.24 & 0.235 & & 0.040 & 0.033 & $U$ & 0.033 & 0.095 & $\mathrm{U}$ & 0.095 & 0.13 & $\mathrm{U}$ & 0.13 \\
\hline Beneath safe & J13DJ5 & $09 / 07 / 06$ & 0.15 & & 0.088 & 0.061 & $\mathrm{U}$ & 0.061 & 0.078 & $U$ & 0.078 & 0.095 & $U$ & 0.095 & 0.22 & $U$ & 0.22 \\
\hline Beneath oil drums & J13DH9 & $09 / 07 / 06$ & 1.38 & & 0.053 & 0.069 & & 0.026 & 0.019 & $U$ & 0.019 & 0.050 & $U$ & 0.050 & 0.062 & $\bar{U}$ & 0.062 \\
\hline
\end{tabular}

\begin{tabular}{|c|c|c|c|c|c|c|c|c|c|c|c|c|c|c|c|c|c|}
\hline \multirow{2}{*}{ Description } & \multirow{2}{*}{$\begin{array}{c}\text { Sample } \\
\text { Number }\end{array}$} & \multirow{2}{*}{$\begin{array}{c}\text { Sample } \\
\text { Date }\end{array}$} & \multicolumn{3}{|c|}{ Europium-155 } & \multicolumn{3}{|c|}{ Nickel-63 } & \multicolumn{3}{|c|}{ Tritium } & \multicolumn{3}{|c|}{ Plutonium-238 } & \multicolumn{3}{|c|}{ Plutonium-239/240 } \\
\hline & & & $\mathrm{pCi} / \mathrm{g}$ & $\mathbf{Q}$ & MDA & $\mathrm{pCi} / \mathrm{g}$ & $\mathbf{Q}$ & MDA & $\mathrm{pCi} / \mathrm{g}$ & $\mathbf{Q}$ & MDA & $\mathrm{pCi} / \mathrm{g}$ & $\mathbf{Q}$ & MDA & $\mathrm{pCi} / \mathrm{g}$ & $\mathbf{Q}$ & MDA \\
\hline \begin{tabular}{|l|} 
Discolored soil \\
\end{tabular} & J13H86 & $09 / 28 / 06$ & 0.076 & $\bar{U}$ & 0.076 & 1.03 & $\mathrm{U}$ & 3.6 & 0.464 & $U$ & 2.6 & 0 & $U$ & 0.30 & 0.237 & $\mathrm{U}$ & 0.30 \\
\hline Discolored soil & J13H87 & $09 / 28 / 06$ & 0.14 & $\mathrm{U}$ & 0.14 & -0.486 & $\mathrm{U}$ & 3.7 & 0.548 & $\bar{U}$ & 2.7 & 0.089 & $U$ & 0.34 & 1.12 & & 0.34 \\
\hline Hot spot & J13DNO & $09 / 26 / 06$ & 0.29 & $\mathrm{U}$ & 0.29 & 1.08 & $\mathrm{U}$ & 3.5 & -0.232 & $U$ & 2.7 & 0.155 & $U$ & 0.50 & 0.207 & $\mathrm{U}$ & 0.40 \\
\hline Hot spot & J13DN1 & $09 / 26 / 06$ & 0.093 & $U$ & 0.093 & -0.302 & $U$ & 3.3 & 0.844 & $U$ & 2.7 & 0.526 & & 0.42 & 79.7 & & 0.33 \\
\hline Hot spot & J13DN2 & $09 / 26 / 06$ & 0.092 & $\mathrm{U}$ & 0.092 & 0.306 & $\mathrm{U}$ & 3.6 & 0.236 & $U$ & 2.7 & 1.57 & & 0.48 & 91.6 & & 0.48 \\
\hline Hot spot & J13DN3 & $09 / 26 / 06$ & 0.082 & $U$ & 0.082 & 0.391 & $\mathrm{U}$ & 3.5 & -1.24 & $u$ & 2.8 & -0.058 & $u$ & 0.44 & 0 & $\mathrm{U}$ & 0.44 \\
\hline Hot spot & J13DN4 & $09 / 26 / 06$ & 0.082 & $\bar{U}$ & 0.082 & -0.174 & $U$ & 3.5 & -1.64 & $U$ & 2.9 & -0.081 & U & 0.62 & 0 & $\mathrm{U}$ & 0.62 \\
\hline Beneath safe & J13DJ5 & $09 / 07 / 06$ & 0.062 & $\bar{U}$ & 0.062 & -0.243 & $\bar{U}$ & 3.6 & 0.852 & $U$ & 2.6 & 0.088 & $\mathrm{U}$ & 0.42 & 1.58 & & 0.34 \\
\hline Beneath oil drums & $\mathrm{J13DH} 9$ & $09 / 07 / 06$ & 0.047 & $U$ & 0.047 & 1.29 & $U$ & 3.8 & 1.79 & $U$ & 2.6 & 0.142 & $\mathrm{U}$ & 0.54 & 21.1 & & 0.54 \\
\hline
\end{tabular}

\begin{tabular}{|c|c|c|c|c|c|c|c|c|c|c|c|c|c|c|c|c|c|}
\hline \multirow{2}{*}{ Description } & \multirow{2}{*}{$\begin{array}{l}\text { Sample } \\
\text { Number }\end{array}$} & \multirow{2}{*}{$\begin{array}{c}\text { Sample } \\
\text { Date }\end{array}$} & \multicolumn{3}{|c|}{ Plutonium-241 } & \multicolumn{3}{|c|}{ Strontium-90 } & \multicolumn{3}{|c|}{ Uranium-233/234 } & \multicolumn{3}{|c|}{ Uranium-235 } & \multicolumn{3}{|c|}{ Uranium-238 } \\
\hline & & & $\mathrm{pCi} / \mathrm{g}$ & $\mathbf{Q}$ & MDA & $\mathrm{pCi} / \mathrm{g}$ & $\mathbf{Q}$ & MDA & $\mathrm{pCi} / \mathrm{g}$ & $\mathbf{Q}$ & MDA & $\mathrm{pCi} / \mathrm{g}$ & $\mathbf{Q}$ & MDA & $\mathrm{pCi} / \mathrm{g}$ & $\mathbf{Q}$ & MDA \\
\hline Discolored soil & $\mathrm{J} 13 \mathrm{H} 86$ & $09 / 28 / 06$ & 3.93 & $\mathrm{U}$ & 20 & 0.014 & $U$ & 0.29 & 6.37 & & 0.22 & 0.465 & & 0.21 & 6.28 & & 0.22 \\
\hline Discolored soil & J13H87 & $09 / 28 / 06$ & 10.2 & $\mathrm{U}$ & 23 & 0.038 & $\mathrm{U}$ & 0.22 & 7.24 & & 0.20 & 0.520 & & 0.19 & 6.71 & & 0.20 \\
\hline Hot spot & J13DNO & $09 / 26 / 06$ & 1.04 & $\mathrm{U}$ & 18 & 7.09 & & 0.22 & 47.8 & & 0.42 & 2.42 & & 0.23 & 50.1 & & 0.40 \\
\hline Hot spot & J13DN1 & $09 / 26 / 06$ & 12.3 & $\mathrm{U}$ & 17 & 0.462 & & 0.23 & 8.99 & & 0.23 & 0.473 & & 0.23 & 10.3 & & 0.23 \\
\hline Hot spot & J13DN2 & $09 / 26 / 06$ & 32.9 & & 17 & 7.20 & & 0.23 & 13.4 & & 0.28 & 0.695 & & 0.23 & 13.4 & & 0.24 \\
\hline Hot spot & J13DN3 & $09 / 26 / 06$ & -6.99 & $\mathrm{U}$ & 19 & 0.006 & $U$ & 0.32 & 0.546 & & 0.20 & 0 & $\mathrm{U}$ & 0.24 & 0.494 & & 0.20 \\
\hline Hot spot & J13DN4 & $09 / 26 / 06$ & 2.10 & $\mathrm{U}$ & 20 & 0.062 & $u$ & 0.32 & 0.497 & & 0.21 & 0.033 & $\mathrm{U}$ & 0.26 & 0.497 & & 0.21 \\
\hline Beneath safe & J13DJ5 & $09 / 07 / 06$ & 6.09 & $\mathrm{U}$ & 18 & 0.375 & & 0.28 & 3.05 & & 0.17 & 0.293 & & 0.20 & 3.52 & & 0.17 \\
\hline Beneath oil drums & J13DH9 & $09 / 07 / 06$ & 19.6 & $\bar{U}$ & 22 & 1.61 & & 0.21 & 9.82 & & 0.25 & 0.438 & & 0.24 & 10.7 & & 0.20 \\
\hline
\end{tabular}


CVP-2006-00010

Rev. 0

Table B-5. 618-2 Verification Sample Results (Biased Samples). (3 pages)

\begin{tabular}{|l|c|c|c|}
\hline \multirow{2}{*}{ Constituents } & \multicolumn{3}{|c|}{$\begin{array}{l}\text { J13DH9 } \\
\text { Beneath oil drums } \\
\mathbf{0 9 / 0 7 / 0 6}\end{array}$} \\
\cline { 2 - 5 } & $\boldsymbol{\mu g} / \mathbf{k g}$ & $\mathbf{Q}$ & PQL \\
\hline 1,2,4-Trichlorobenzene & 340 & $\mathrm{UJ}$ & 340 \\
\hline 1,2-Dichlorobenzene & 340 & $\mathrm{U}$ & 340 \\
\hline 1,3-Dichlorobenzene & 340 & $\mathrm{U}$ & 340 \\
\hline 1,4-Dichlorobenzene & 340 & $\mathrm{U}$ & 340 \\
\hline 2,4,5-Trichlorophenol & 850 & $\mathrm{U}$ & 850 \\
\hline 2,4,6-Trichlorophenol & 340 & $\mathrm{U}$ & 340 \\
\hline 2,4-Dichlorophenol & 340 & $\mathrm{U}$ & 340 \\
\hline 2,4-Dimethylphenol & 340 & $\mathrm{UJ}$ & 340 \\
\hline 2,4-Dinitrophenol & 850 & $\mathrm{U}$ & 850 \\
\hline 2,4-Dinitrotoluene & 340 & $\mathrm{U}$ & 340 \\
\hline 2,6-Dinitrotoluene & 340 & $\mathrm{U}$ & 340 \\
\hline 2-Chloronaphthalene & 340 & $\mathrm{U}$ & 340 \\
\hline 2-Chlorophenol & 340 & $\mathrm{U}$ & 340 \\
\hline 2-Methylnaphthalene & 340 & $\mathrm{UJ}$ & 340 \\
\hline 2-Methylphenol (cresol, o-) & 340 & $\mathrm{UJ}$ & 340 \\
\hline 2-Nitroaniline & 850 & $\mathrm{U}$ & 850 \\
\hline 2-Nitrophenol & 340 & $\mathrm{U}$ & 340 \\
\hline 3+4 Methylphenol (cresol, $\mathrm{m+p}$ ) & 340 & $\mathrm{UJ}$ & 340 \\
\hline 3,3'-Dichlorobenzidine & 340 & $\mathrm{U}$ & 340 \\
\hline 3-Nitroaniline & 850 & $\mathrm{U}$ & 850 \\
\hline 4,6-Dinitro-2-methylphenol & 850 & $\mathrm{U}$ & 850 \\
\hline 4-Bromophenylphenyl ether & 340 & $\mathrm{U}$ & 340 \\
\hline 4-Chloro-3-methylphenol & 340 & $\mathrm{UJ}$ & 340 \\
\hline 4-Chloroaniline & 340 & $\mathrm{U}$ & 340 \\
\hline 4-Chlorophenylphenyl ether & 340 & $\mathrm{U}$ & 340 \\
\hline 4-Nitroaniline & 850 & $\mathrm{U}$ & 850 \\
\hline 4-Nitrophenol & 850 & $\mathrm{U}$ & 850 \\
\hline Acenaphthene & 340 & $\mathrm{U}$ & 340 \\
\hline Acenaphthylene & 340 & $\mathrm{U}$ & 340 \\
\hline Anthracene & 340 & $\mathrm{U}$ & 340 \\
\hline Benzo(a)anthracene & 340 & $\mathrm{U}$ & 340 \\
\hline Benzo(a)pyrene & 340 & $\mathrm{U}$ & 340 \\
\hline
\end{tabular}

\begin{tabular}{|c|c|c|c|}
\hline \multirow[t]{2}{*}{ Constituents } & \multicolumn{3}{|c|}{$\begin{array}{c}\text { J13DH9 } \\
\text { Beneath oil drums } \\
09 / 07 / 06\end{array}$} \\
\hline & $\mu \mathrm{g} / \mathrm{kg}$ & $\mathbf{Q}$ & PQL \\
\hline Benzo(b)fluoranthene & 340 & $\mathrm{U}$ & 340 \\
\hline Benzo(ghi)perylene & 340 & U & 340 \\
\hline Benzo(k)fluoranthene & 340 & $\mathrm{U}$ & 340 \\
\hline bis(2-Chloro-1-methylethyl)ether & 340 & $\mathrm{U}$ & 340 \\
\hline bis(2-Chloroethoxy)methane & 340 & $\mathrm{U}$ & 340 \\
\hline bis(2-Chloroethyl) ether & 340 & $\mathrm{U}$ & 340 \\
\hline bis(2-Ethylhexyl) phthalate & 27 & $\mathrm{~J}$ & 340 \\
\hline Butylbenzylphthalate & 340 & $\mathrm{U}$ & 340 \\
\hline Carbazole & 340 & $\mathrm{U}$ & 340 \\
\hline Chrysene & 340 & $\mathrm{U}$ & 340 \\
\hline Di-n-butylphthalate & 18 & $\mathrm{~J}$ & 340 \\
\hline Di-n-octylphthalate & 340 & $\mathrm{U}$ & 340 \\
\hline Dibenz[a,h]anthracene & 340 & $\mathrm{U}$ & 340 \\
\hline Dibenzofuran & 340 & $\mathrm{U}$ & 340 \\
\hline Diethylphthalate & 340 & $\mathrm{U}$ & 340 \\
\hline Dimethyl phthalate & 340 & $\mathrm{U}$ & 340 \\
\hline Fluoranthene & 340 & $\mathrm{U}$ & 340 \\
\hline Fluorene & 340 & $\mathrm{U}$ & 340 \\
\hline Hexachlorobenzene & 340 & $\mathrm{U}$ & 340 \\
\hline Hexachlorobutadiene & 340 & $\mathrm{U}$ & 340 \\
\hline Hexachlorocyclopentadiene & 340 & $\mathrm{U}$ & 340 \\
\hline Hexachloroethane & 340 & $\mathrm{U}$ & 340 \\
\hline Indeno(1,2,3-cd)pyrene & 340 & $\mathrm{U}$ & 340 \\
\hline Isophorone & 340 & $\mathrm{UJ}$ & 340 \\
\hline N-Nitroso-di-n-dipropylamine & 340 & $\mathrm{U}$ & 340 \\
\hline N-Nitrosodiphenylamine & 340 & $\mathrm{U}$ & 340 \\
\hline Naphthalene & 340 & $\mathrm{U}$ & 340 \\
\hline Nitrobenzene & 340 & UJ & 340 \\
\hline Pentachlorophenol & 850 & $\mathrm{U}$ & 850 \\
\hline Phenanthrene & 340 & $\mathrm{U}$ & 340 \\
\hline Phenol & 340 & $\mathrm{U}$ & 340 \\
\hline Pyrene & 340 & $\mathrm{U}$ & 340 \\
\hline
\end{tabular}


Table B-6. Test Pit Sample Results. (4 Pages)

\begin{tabular}{|c|c|c|c|c|c|c|c|c|c|c|c|c|c|c|c|c|c|c|}
\hline \multirow[b]{2}{*}{ Description } & \multirow{2}{*}{\begin{tabular}{|c|} 
Sample \\
Elevation \\
(m)
\end{tabular}} & \multirow{2}{*}{$\begin{array}{c}\text { Sample } \\
\text { Number }\end{array}$} & \multirow{2}{*}{$\begin{array}{c}\text { Sample } \\
\text { Date }\end{array}$} & \multicolumn{3}{|c|}{ Arsenic } & \multicolumn{3}{|c|}{ Barium } & \multicolumn{3}{|c|}{ Beryllium } & \multicolumn{3}{|c|}{ Cadmium } & \multicolumn{3}{|c|}{ Chromium } \\
\hline & & & & $\mathrm{mg} / \mathrm{kg}$ & $\mathbf{Q}$ & PQL & mg/kg & Q & PQL & $\mathrm{mg} / \mathrm{kg}$ & Q & PQL & $\mathrm{mg} / \mathrm{kg}$ & $\mathbf{Q}$ & PQL & $\mathrm{mg} / \mathrm{kg}$ & Q & PQL \\
\hline Soil & 111 & J12NK9 & $6 / 19 / 06$ & 1 & & 0.62 & 88.4 & & 0.02 & 0.17 & & 0.02 & 1.5 & & 0.07 & 6.7 & & 0.13 \\
\hline Soil & 110.5 & J12NL3 & $7 / 12 / 06$ & 0.99 & & 0.61 & 102 & & 0.02 & 0.03 & & 0.02 & 1.6 & & 0.07 & 3.9 & & 0.13 \\
\hline Soil & 110 & H12Y08 & $8 / 2 / 06$ & 1.5 & & 0.61 & 72.3 & & 0.02 & 0.02 & $\mathrm{U}$ & 0.02 & 2.5 & & 0.07 & 1.9 & & 0.13 \\
\hline Soil & 109 & J12Y09 & $8 / 2 / 06$ & 1.3 & & 0.60 & 47.9 & & 0.02 & 0.02 & U & 0.02 & 0.64 & & 0.07 & 4.6 & & 0.13 \\
\hline Soil & 108 & $\mathrm{~J} 12 \mathrm{Y} 10$ & $8 / 2 / 06$ & 1.6 & & 0.61 & 47.8 & & 0.02 & 0.18 & & 0.02 & 0.89 & & 0.07 & 3.5 & & 0.13 \\
\hline Soil & 107 & $\mathrm{~J} 12 \mathrm{Y} 11$ & $8 / 2 / 06$ & 2.3 & & 0.64 & 60.2 & & 0.02 & 0.11 & & 0.02 & 1.1 & & 0.07 & 3.7 & & 0.14 \\
\hline Soil & 106 & $\mathrm{~J} 134 \mathrm{~V} 2$ & $8 / 2 / 06$ & 7.5 & & 0.73 & 123 & & 0.02 & 0.42 & & 0.02 & 0.38 & & 0.08 & 8.7 & & 0.16 \\
\hline Soil & 105 & $\mathrm{~J} 134 \mathrm{~V} 3$ & $8 / 2 / 06$ & 2.1 & & 0.74 & 84.5 & & 0.02 & 0.91 & & 0.02 & 0.60 & & 0.08 & 10.4 & & 0.16 \\
\hline \multicolumn{4}{|c|}{ Water Samples } & $\mathrm{ug} / \mathrm{L}$ & $\mathbf{Q}$ & PQL & ug/L & $\mathbf{Q}$ & PQL & $\mathrm{ug} / \mathrm{L}$ & Q & PQL & $\mathrm{ug} / \mathrm{L}$ & $\mathbf{Q}$ & PQL & $\mathrm{ug} / \mathrm{L}$ & Q & PQL \\
\hline Water (Filtered) & 104.5 & $\mathrm{~J} 12 \mathrm{Y} 73$ & $8 / 2 / 06$ & 6.1 & $U$ & 6.1 & 229 & & 229 & 0.20 & U & 0.20 & 421 & & 0.70 & 1.3 & U & 1.3 \\
\hline Water (nonfiltered) & 104.5 & J12Y74 & $8 / 2 / 06$ & 738 & & 30.5 & 38,300 & & 1.0 & 95.3 & & 1.0 & 2380 & & 3.5 & 2180 & & 6.5 \\
\hline
\end{tabular}

\begin{tabular}{|c|c|c|c|c|c|c|c|c|c|c|c|c|c|c|c|c|c|c|}
\hline \multirow[b]{2}{*}{ Description } & \multirow{2}{*}{\begin{tabular}{|c|} 
Sample \\
Elevation \\
$(\mathrm{m})$ \\
\end{tabular}} & \multirow{2}{*}{$\begin{array}{l}\text { Sample } \\
\text { Number }\end{array}$} & \multirow{2}{*}{$\begin{array}{c}\text { Sample } \\
\text { Date }\end{array}$} & \multicolumn{3}{|c|}{ Lead } & \multicolumn{3}{|c|}{ Selenium } & \multicolumn{3}{|c|}{ Silver } & \multicolumn{3}{|c|}{ Tin } & \multicolumn{3}{|c|}{ Uranium } \\
\hline & & & & $\mathrm{mg} / \mathrm{kg}$ & $\mathbf{Q}$ & PQL & $\mathrm{mg} / \mathrm{kg}$ & $Q$ & PQL & $\mathrm{mg} / \mathrm{kg}$ & $\mathbf{Q}$ & PQL & $\mathrm{mg} / \mathrm{kg}$ & $\mathbf{Q}$ & PQL & $\mathrm{mg} / \mathrm{kg}$ & $Q$ & PQL \\
\hline Soil & 111 & J12NK9 & $6 / 19 / 06$ & 29.6 & & 0.31 & 0.48 & U & 0.48 & 0.07 & U & 0.07 & 2.9 & & 1.1 & 28.1 & & 0.89 \\
\hline Soil & 110.5 & J12NL3 & $7 / 12 / 06$ & 3.4 & & 0.31 & 0.47 & $\mathrm{U}$ & 0.47 & 0.07 & U & 0.07 & 1.1 & $U$ & 1.1 & 13.0 & & 0.88 \\
\hline Soil & 110 & H12Y08 & $8 / 2 / 06$ & 2.0 & & 0.31 & 0.47 & $U$ & 0.47 & 0.07 & $U$ & 0.07 & 1.1 & $\mathrm{U}$ & 1.1 & 74.2 & & 0.88 \\
\hline Soil & 109 & J12Y09 & $8 / 2 / 06$ & 2.6 & & 0.31 & 0.46 & $\mathrm{U}$ & 0.46 & 0.07 & U & 0.07 & 1.1 & $\mathrm{U}$ & 1.1 & 12.8 & & 0.87 \\
\hline Soil & 108 & $\mathrm{~J} 12 \mathrm{Y} 10$ & $8 / 2 / 06$ & 2.7 & & 0.31 & 0.47 & $\mathrm{U}$ & 0.47 & 0.07 & U & 0.07 & 1.1 & $U$ & 1.1 & 33.9 & & 0.89 \\
\hline Soil & 107 & $\mathrm{~J} 12 \mathrm{Y} 11$ & $8 / 2 / 06$ & 2.6 & & 0.33 & 0.50 & $\mathrm{U}$ & 0.50 & 0.07 & U & 0.07 & 1.1 & 1 & 1.1 & 18.0 & & 0.93 \\
\hline Soil & 106 & $\mathrm{~J} 134 \mathrm{~V} 2$ & $8 / 2 / 06$ & 4.9 & & 0.37 & 0.56 & U & 0.56 & 0.08 & $U$ & 0.08 & 1.3 & $\mathrm{U}$ & 1.3 & 20.5 & & 1.1 \\
\hline Soil & 105 & J134V3 & $8 / 2 / 06$ & 12.2 & & 0.38 & 0.57 & $\mathrm{U}$ & 0.57 & 0.08 & $U$ & 0.08 & 1.3 & $\mathrm{U}$ & 1.3 & 4.1 & & 1.1 \\
\hline \multicolumn{4}{|c|}{ Water Samples } & $\mathrm{ug} / \mathrm{L}$ & $Q$ & PQL & $u g / L$ & $\mathbf{Q}$ & PQL & ug/L & $Q$ & $\overline{P Q L}$ & $\mathrm{ug} / \mathrm{L}$ & $Q$ & PQL & ug/L & Q & PQL \\
\hline Water (Filtered) & 104.5 & J12Y73 & $8 / 2 / 06$ & 3.1 & U & 3.1 & 5.2 & & 4.7 & & $\mathrm{NA}$ & & & NA & & & $\mathrm{NA}$ & \\
\hline Water (nonfiltered) & 104.5 & $\mathrm{~J} 12 \mathrm{Y} 74$ & $8 / 2 / 06$ & 1000 & & 15.5 & 23.5 & $\mathrm{U}$ & 23.5 & & $\mathrm{NA}$ & & & $\mathrm{NA}$ & & & $\mathrm{NA}$ & \\
\hline
\end{tabular}


Table B-6. Test Pit Sample Results. (4 pages)

\begin{tabular}{|c|c|c|c|c|c|c|c|c|c|c|c|c|c|c|c|}
\hline \multirow{2}{*}{ Description } & \multirow{2}{*}{$\begin{array}{l}\text { Sample } \\
\text { Elevation } \\
\text { (m) }\end{array}$} & \multirow{2}{*}{$\begin{array}{l}\text { Sample } \\
\text { Number }\end{array}$} & \multirow{2}{*}{$\begin{array}{c}\text { Sample } \\
\text { Date }\end{array}$} & \multicolumn{3}{|c|}{ Bromide } & \multicolumn{3}{|c|}{ Chloride } & \multicolumn{3}{|c|}{ Flouride } & \multicolumn{3}{|c|}{ Nitrite } \\
\hline & & & & $\mathrm{mg} / \mathrm{kg}$ & $\mathbf{Q}$ & PQL & $\mathrm{mg} / \mathrm{kg}$ & $Q$ & PQL & $\mathrm{mg} / \mathrm{kg}$ & $Q$ & PQL & $\mathrm{mg} / \mathrm{kg}$ & $\mathbf{Q}$ & PQL \\
\hline Soil & 111 & J12NK9 & $6 / 19 / 06$ & \multicolumn{3}{|c|}{ NA } & \multicolumn{3}{|c|}{ NA } & \multicolumn{3}{|c|}{$\overline{N A}$} & \multicolumn{3}{|c|}{$\mathrm{NA}$} \\
\hline Soil & 110.5 & J12NL3 & $7 / 12 / 06$ & \multicolumn{3}{|c|}{$\mathrm{NA}$} & \multicolumn{3}{|c|}{ NA } & \multicolumn{3}{|c|}{$\overline{N A}$} & \multicolumn{3}{|c|}{ NA } \\
\hline Soil & 110 & H12Y08 & $8 / 2 / 06$ & 2.5 & $U$ & 2.5 & 287 & & 25.3 & 2.5 & $\mathrm{U}$ & 2.5 & 2.53 & $U$ & 2.53 \\
\hline Soil & 109 & J12Y09 & $8 / 2 / 06$ & 3.7 & & 2.6 & 622 & & 25.6 & 2.6 & $\mathrm{U}$ & 2.6 & 2.56 & $U$ & 2.56 \\
\hline Soil & 108 & $\mathrm{~J} 12 \mathrm{Y} 10$ & $8 / 2 / 06$ & 2.8 & & 2.5 & 515 & & 24.9 & 7.0 & & 2.5 & 2.49 & $\mathrm{U}$ & 2.49 \\
\hline Soil & 107 & $\mathrm{~J} 12 \mathrm{Y} 11$ & $8 / 2 / 06$ & 2.7 & $\mathrm{U}$ & 2.7 & 329 & & 27.4 & 2.7 & $\mathrm{U}$ & 2.7 & 2.74 & $\mathrm{U}$ & 2.74 \\
\hline Soil & 106 & J134V2 & $8 / 2 / 06$ & 10.6 & & 3.1 & 2620 & & 77.7 & 3.1 & $U$ & 3.1 & 15.5 & & 15.5 \\
\hline Soil & 105 & $\mathrm{~J} 134 \mathrm{~V} 3$ & $8 / 2 / 06$ & 6.9 & & 3.2 & 3620 & & 158 & 3.2 & $\mathrm{U}$ & 3.2 & 31.5 & $\bar{U}$ & 31.5 \\
\hline Water (Filtered) & 104.5 & $\mathrm{J12Y73}$ & $8 / 2 / 06$ & \multicolumn{3}{|c|}{$\mathrm{NA}$} & \multicolumn{3}{|c|}{ NA } & \multicolumn{3}{|c|}{ NA } & \multicolumn{3}{|c|}{ NA } \\
\hline Water (nonfiltered) & 104.5 & J12Y74 & $8 / 2 / 06$ & \multicolumn{3}{|c|}{$\overline{\mathrm{NA}}$} & \multicolumn{3}{|c|}{$\overline{N A}$} & \multicolumn{3}{|c|}{ NA } & \multicolumn{3}{|c|}{$\mathrm{NA}$} \\
\hline
\end{tabular}

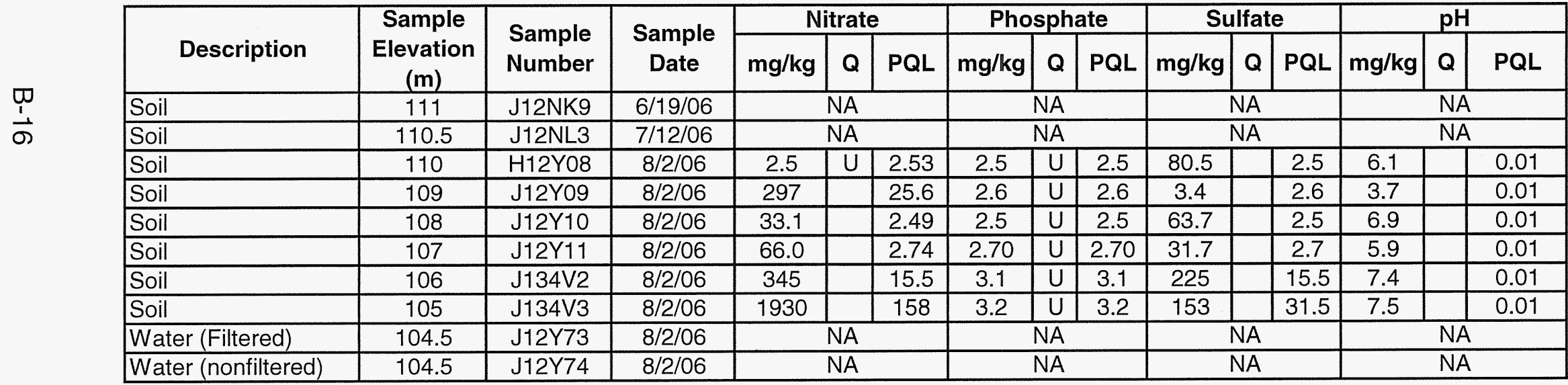


Table B-6. Test Pit Sample Results. (4 pages)

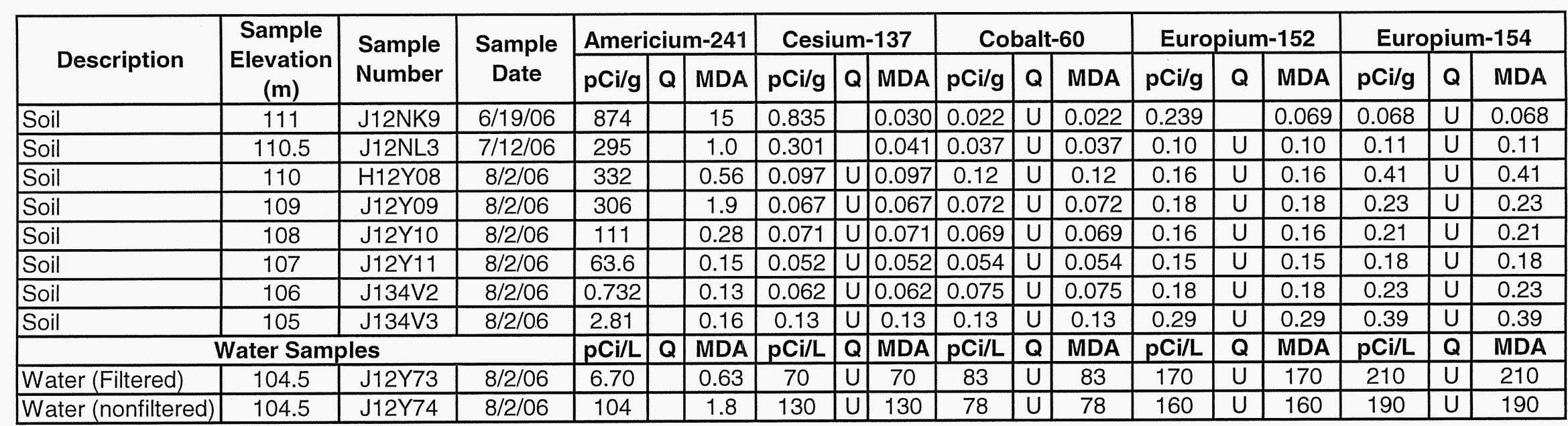

\begin{tabular}{|c|c|c|c|c|c|c|c|c|c|c|c|c|c|c|c|c|c|c|}
\hline \multirow{2}{*}{ Description } & \multirow{2}{*}{$\begin{array}{c}\text { Sample } \\
\text { Elevation } \\
(\mathrm{m})\end{array}$} & \multirow{2}{*}{$\begin{array}{l}\text { Sample } \\
\text { Number }\end{array}$} & \multirow{2}{*}{$\begin{array}{c}\text { Sample } \\
\text { Date }\end{array}$} & \multicolumn{3}{|c|}{ Europium-155 } & \multicolumn{3}{|c|}{ Nickel-63 } & \multicolumn{3}{|c|}{ Tritium } & \multicolumn{3}{|c|}{ Plutonium-238 } & \multicolumn{3}{|c|}{ Plutonium-239/240 } \\
\hline & & & & $\mathrm{pCi} / \mathrm{g}$ & $\mathbf{Q}$ & MDA & $\mathrm{pCi} / \mathrm{g}$ & $Q$ & MDA & $\mathrm{pCi} / \mathrm{g}$ & $\mathbf{Q}$ & MDA & $\mathrm{pCi} / \mathrm{g}$ & $\mathbf{Q}$ & MDA & $\mathrm{pCi} / \mathrm{g}$ & $\mathbf{Q}$ & MDA \\
\hline Soil & 111 & J12NK9 & $6 / 19 / 06$ & 0.17 & $U$ & 0.17 & -3.31 & $\mathrm{U}$ & 21 & 0.978 & $\mathrm{U}$ & 2.7 & 163 & & 34 & 13,500 & & 27 \\
\hline Soil & 110.5 & J12NL3 & $7 / 12 / 06$ & 0.17 & $\mathrm{U}$ & 0.17 & -1.26 & $U$ & 11 & 2.85 & & 2.8 & 38.7 & & 13 & 2,350 & & 13 \\
\hline Soil & 110 & $\mathrm{H} 12 \mathrm{Y08}$ & $8 / 2 / 06$ & 0.14 & $\mathrm{U}$ & 0.14 & 6.52 & $\mathrm{U}$ & 16 & 1.05 & $\mathrm{U}$ & 2.6 & 1.47 & & 0.45 & 57.2 & & 0.36 \\
\hline Soil & 109 & J12Y09 & $8 / 2 / 06$ & 0.23 & $\mathrm{U}$ & 0.23 & 9.29 & $\mathrm{U}$ & 50 & 3.57 & & 2.7 & 21.1 & & 3.9 & 1710 & & 2.2 \\
\hline Soil & 108 & $\mathrm{~J} 12 \mathrm{Y} 10$ & $8 / 2 / 06$ & 0.20 & $\mathrm{U}$ & 0.20 & 1.32 & $\mathrm{U}$ & 3.6 & 2.38 & $\mathrm{U}$ & 2.4 & 2.39 & & 0.11 & 150 & & 0.076 \\
\hline Soil & 107 & $\mathrm{~J} 12 \mathrm{Y} 11$ & $8 / 2 / 06$ & 0.13 & $U$ & 0.13 & -0.54 & $\mathrm{U}$ & 3.50 & 2.09 & $\mathrm{U}$ & 2.6 & 0.827 & & 0.10 & 62.1 & & 0.071 \\
\hline Soil & 106 & J134V2 & $8 / 2 / 06$ & 0.27 & $\mathrm{U}$ & 0.27 & 1.39 & $\mathrm{U}$ & 3.7 & 5.79 & & 2.4 & 0.049 & $\mathrm{U}$ & 0.049 & 5.62 & & 0.075 \\
\hline Soil & 105 & J134V3 & $8 / 2 / 06$ & 0.33 & $U$ & 0.33 & 2.14 & $\bar{U}$ & 3.1 & 12.9 & & 2.5 & 0 & $\mathrm{U}$ & 0.15 & 0.918 & & 0.075 \\
\hline \multicolumn{4}{|c|}{ Water Samples } & $\mathrm{pCi} / \mathrm{L}$ & $\mathbf{Q}$ & MDA & $\mathrm{pCi} / \mathrm{L}$ & $\mathbf{Q}$ & MDA & $\mathrm{pCi} / \mathrm{L}$ & $\mathbf{Q}$ & MDA & $\mathrm{pCi} / \mathrm{L}$ & $\mathbf{Q}$ & MDA & $\mathrm{pCi} / \mathrm{L}$ & $\mathbf{Q}$ & MDA \\
\hline Water (Filtered) & 104.5 & J12Y73 & $8 / 2 / 06$ & 180 & $U$ & 180 & 13 & $U$ & 16 & 4890 & & 160 & -0.060 & $\mathrm{U}$ & 0.81 & 2.96 & & 0.46 \\
\hline Water (nonfiltered) & 104.5 & $\mathrm{~J} 12 \mathrm{Y} 74$ & $8 / 2 / 06$ & 170 & $\mathrm{U}$ & 170 & -1.84 & $\mathrm{U}$ & 15 & 7830 & & 160 & 0.188 & $\mathrm{U}$ & 0.52 & 20 & & 0.36 \\
\hline
\end{tabular}


Table B-6. Test Pit Sample Results. (4 pages)

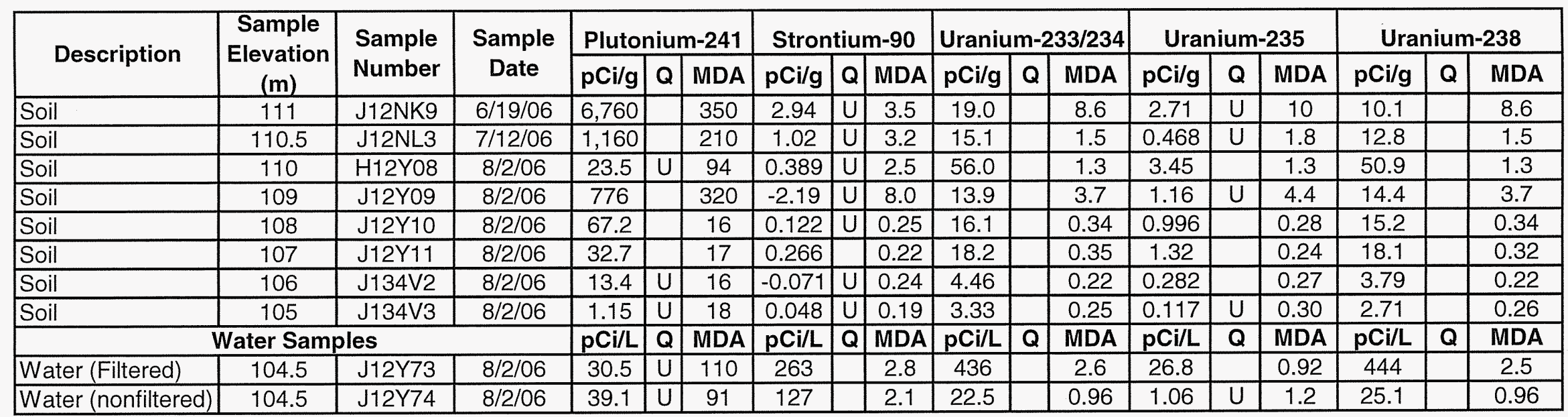

$\frac{1}{1}$ 
Table B-7. Pothole Sample Results. (2 pages)

\begin{tabular}{|c|c|c|c|c|c|c|c|c|c|c|c|c|c|c|c|c|c|c|}
\hline \multirow[b]{2}{*}{ Description } & \multirow{2}{*}{$\begin{array}{l}\text { Sample } \\
\text { Elevation } \\
(\mathrm{m})\end{array}$} & \multirow{2}{*}{$\begin{array}{l}\text { Sample } \\
\text { Number }\end{array}$} & \multirow{2}{*}{$\begin{array}{c}\text { Sample } \\
\text { Date }\end{array}$} & \multicolumn{3}{|c|}{ Arsenic } & \multicolumn{3}{|c|}{ Barium } & \multicolumn{3}{|c|}{ Beryllium } & \multicolumn{3}{|c|}{ Cadmium } & \multicolumn{3}{|c|}{ Chromium } \\
\hline & & & & $\mathrm{mg} / \mathrm{kg}$ & $\mathbf{Q}$ & PQL & $\mathrm{mg} / \mathrm{kg}$ & $\mathbf{Q}$ & PQL & $\mathrm{mg} / \mathrm{kg}$ & $\mathbf{Q}$ & PQL & $\mathrm{mg} / \mathrm{kg}$ & $\mathbf{Q}$ & PQL & $\mathrm{g} / \mathrm{kg}$ & $\mathbf{Q}$ & PQL \\
\hline Nort & 108 & J13H52 & 9 & 1.1 & & 0.37 & $\overline{93}$ & & 0.02 & 0.25 & & 0.01 & 0.06 & & 0.03 & 2.7 & & 0.07 \\
\hline South & & & $9 / 25$ & 1.3 & & 0.36 & 55.5 & & 0.02 & 0.30 & & 0.01 & 0.05 & & 0.03 & 3.8 & & 0.07 \\
\hline Northws & 108 & J13H54 & $9 / 25 / 06$ & 1.5 & & 0.35 & 52.3 & & 0.02 & 0.32 & & 0.01 & 0.07 & & 0.03 & 3.9 & & 0.07 \\
\hline Southwest corner & 108 & J13H55 & $9 / 25 / 06$ & 1.5 & & 0.37 & 69.4 & & 0.02 & 0.39 & & 0.01 & 2.7 & & 0.03 & 5.3 & & 0.07 \\
\hline
\end{tabular}

\begin{tabular}{|c|c|c|c|c|c|c|c|c|c|c|c|c|c|c|c|c|c|c|}
\hline \multirow[b]{2}{*}{ Description } & \multirow{2}{*}{$\begin{array}{c}\text { Sample } \\
\text { Elevation } \\
(\mathrm{m})\end{array}$} & \multirow{2}{*}{$\begin{array}{c}\text { Sample } \\
\text { Number }\end{array}$} & \multirow{2}{*}{$\begin{array}{c}\text { Sample } \\
\text { Date }\end{array}$} & \multicolumn{3}{|c|}{ Lead } & \multicolumn{3}{|c|}{ Selenium } & \multicolumn{3}{|c|}{ Silver } & \multicolumn{3}{|c|}{ Tin } & \multicolumn{3}{|c|}{ Uranium } \\
\hline & & & & $\mathrm{mg} / \mathrm{kg}$ & $\mathbf{Q}$ & $P Q L$ & $\mathrm{mg} / \mathrm{kg}$ & $\mathbf{Q}$ & $P Q L$ & $\mathrm{mg} / \mathrm{kg}$ & Q & PQL & $\mathrm{g} / \mathrm{kg}$ & $\mathbf{Q}$ & PQL & $\mathrm{g} / \mathbf{k g}$ & $\mathbf{Q}$ & PQL \\
\hline 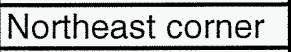 & & $\mathrm{J} 13 \mathrm{H} 52$ & 9 & 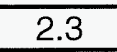 & & 12 & 0.26 & $\mathrm{U}$ & 0.26 & 0.11 & $\mathrm{U}$ & 0.11 & 0.92 & & 0.35 & 2.1 & $\mathrm{U}$ & 2.1 \\
\hline South & & & & 11.2 & & 0.12 & 0.26 & $\mathrm{U}$ & 0.26 & 0.11 & $U$ & 0.11 & 0.62 & & 0.34 & 5.4 & & 2.1 \\
\hline North & 108 & & $9 / 25 / 06$ & 2.5 & & 0.11 & 0.25 & $\mathrm{U}$ & 0.25 & 0.11 & $U$ & 0.11 & 0.72 & & 0.33 & 2 & $U$ & 2 \\
\hline Southwest corner & 108 & J13H55 & $9 / 25 / 06$ & 2.5 & & 0.12 & 0.26 & $\bar{U}$ & 0.26 & 0.11 & $\bar{U}$ & 0.11 & 0.79 & & 0.35 & 2.1 & $\bar{U}$ & 2.1 \\
\hline
\end{tabular}

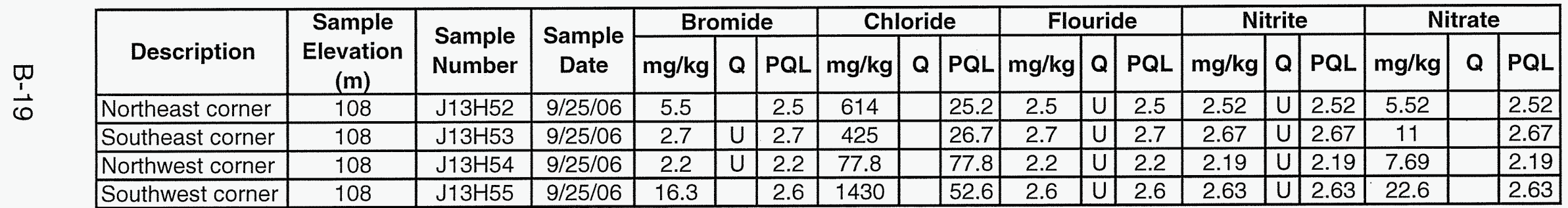

\begin{tabular}{|c|c|c|c|c|c|c|c|c|c|c|c|c|}
\hline \multirow[b]{2}{*}{ Description } & \multirow{2}{*}{$\begin{array}{c}\text { Sample } \\
\text { Elevation } \\
(\mathrm{m})\end{array}$} & \multirow{2}{*}{$\begin{array}{l}\text { Sample } \\
\text { Number }\end{array}$} & \multirow{2}{*}{$\begin{array}{c}\text { Sample } \\
\text { Date }\end{array}$} & \multicolumn{3}{|c|}{ Phosphate } & \multicolumn{3}{|c|}{ Sulfate } & \multicolumn{3}{|c|}{$\mathrm{pH}$} \\
\hline & & & & g & $\mathbf{Q}$ & PQL & $\mathrm{mg} / \mathrm{kg}$ & $\mathbf{Q}$ & PQL & $\mathrm{mg} / \mathrm{kg}$ & $Q$ & PQL \\
\hline Nort & 108 & & & 2.5 & U & 2.5 & 6 & & 2.5 & 8 & & 0.01 \\
\hline Sout & & & & 2.7 & $U$ & 2.7 & 33.9 & & 2.7 & 7.9 & & 0.01 \\
\hline Nort & 10 & & & 2.2 & U & 2.2 & 9.7 & & 2.2 & 8.4 & & 0.01 \\
\hline Southwest corner & 108 & $\mathrm{~J} 13 \mathrm{H} 55$ & $9 / 25 / 06$ & 2.6 & $U$ & 2.6 & 108 & & 52.6 & 6 & & 0.01 \\
\hline
\end{tabular}


Table B-7. Pothole Sample Results. (2 pages)

\begin{tabular}{|c|c|c|c|c|c|c|c|c|c|c|c|c|c|c|c|c|c|c|}
\hline \multirow[b]{2}{*}{ Description } & \multirow{2}{*}{\begin{tabular}{|c|} 
Sample \\
Elevation \\
$(\mathrm{m})$
\end{tabular}} & \multirow{2}{*}{$\begin{array}{l}\text { Sample } \\
\text { Number }\end{array}$} & \multirow{2}{*}{$\begin{array}{c}\text { Sample } \\
\text { Date }\end{array}$} & \multicolumn{3}{|c|}{ Americium-241 } & \multicolumn{3}{|c|}{ Cesium-137 } & \multicolumn{3}{|c|}{ Cobalt-60 } & \multicolumn{3}{|c|}{ Europium-152 } & \multicolumn{3}{|c|}{ Europium-154 } \\
\hline & & & & $\mathrm{pCi} / \mathrm{g}$ & $\mathbf{Q}$ & MDA & $\mathrm{pCi} / \mathrm{g}$ & $\mathbf{Q}$ & MDA & $p C i / g$ & $\mathbf{Q}$ & MDA & $\mathrm{pCi} / \mathrm{g}$ & $\mathbf{Q}$ & MDA & $\mathrm{pCi} / \mathrm{g}$ & $\mathbf{Q}$ & MDA \\
\hline & 100 & & & & & & 91 & $\mathrm{U}$ & & 12 & $U$ & 0.12 & 4 & $u$ & & 39 & J & 9 \\
\hline & & & & 16 & $\mathrm{U}$ & 0.1 & 043 & U & 0.043 & 0.038 & $U$ & 0.038 & 0.10 & $\mathrm{U}$ & 0.10 & 0.12 & $J$ & 0.12 \\
\hline Nor & & & & 0.180 & & 0.17 & 024 & $U$ & 0.024 & 0.026 & $U$ & 0.026 & 0.062 & $U$ & 0.062 & 0.084 & $U$ & 0.084 \\
\hline Southwest corner & 108 & J13H55 & $9 / 25 / 06$ & 38.0 & & 0.16 & 0.041 & $\mathrm{U}$ & 0.041 & \begin{tabular}{|l|}
0.037 \\
\end{tabular} & $U$ & 0.037 & 0.098 & $U$ & 0.098 & 0.13 & $U$ & 0.13 \\
\hline
\end{tabular}

\begin{tabular}{|c|c|c|c|c|c|c|c|c|c|c|c|c|c|c|c|c|c|c|}
\hline \multirow[b]{2}{*}{ Description } & \multirow{2}{*}{$\begin{array}{l}\text { Sample } \\
\text { Elevation } \\
\text { (m) }\end{array}$} & \multirow{2}{*}{$\begin{array}{l}\text { Sample } \\
\text { Number }\end{array}$} & \multirow{2}{*}{$\begin{array}{c}\text { Sample } \\
\text { Date }\end{array}$} & \multicolumn{3}{|c|}{ Europium-155 } & \multicolumn{3}{|c|}{ Nickel-63 } & \multicolumn{3}{|c|}{ Tritium } & \multicolumn{3}{|c|}{ Plutonium-238 } & \multicolumn{3}{|c|}{ Plutonium-239/240 } \\
\hline & & & & $\mathrm{pCi} / \mathrm{g}$ & $Q$ & MDA & $\mathrm{i} / \mathrm{g}$ & Q & MDA & $\mathrm{pCi} / \mathrm{g}$ & $\mathbf{Q}$ & MDA & $/ g$ & $\mathbf{Q}$ & MDA & $\mathrm{Ci} / \mathrm{g}$ & $\mathbf{Q}$ & MDA \\
\hline$\overline{\text { Nort }}$ & 108 & $\bar{J}$ & 6 & 0.090 & $U$ & 0.090 & 0.712 & $U$ & 3.4 & 1.16 & $U$ & 2.6 & 0 & $U$ & 0.44 & 10.0 & & 0.44 \\
\hline Southea & 8 & $\mathrm{~J}$ & $25 / 06$ & 0.095 & $U$ & 0.095 & 1.14 & U & 3.3 & -0.359 & $\mathrm{U}$ & 2.5 & 0 & $U$ & 0.45 & 1.17 & & 0.45 \\
\hline Northwest corner & 108 & 54 & $9 / 25 / 06$ & 0.087 & $\mathrm{U}$ & 0.087 & 0.126 & $U$ & 3.4 & -0.486 & $U$ & 2.7 & 0.066 & $U$ & 0.51 & 0.464 & $U$ & 0.51 \\
\hline Southwest corner & 108 & J13H55 & $9 / 25 / 06$ & 0.091 & $u$ & 0.091 & 0.910 & $\bar{U}$ & 3.3 & -0.530 & $\mathrm{U}$ & 2.6 & 0.102 & $\mathrm{U}$ & 0.97 & 3.97 & & 0.78 \\
\hline
\end{tabular}

\begin{tabular}{|c|c|c|c|c|c|c|c|c|c|c|c|c|c|c|c|c|c|c|}
\hline \multirow[b]{2}{*}{ Description } & \multirow{2}{*}{$\begin{array}{c}\text { Sample } \\
\text { Elevation } \\
(\mathrm{m})\end{array}$} & \multirow{2}{*}{$\begin{array}{c}\text { Sample } \\
\text { Number }\end{array}$} & \multirow{2}{*}{$\begin{array}{c}\text { Sample } \\
\text { Date }\end{array}$} & \multicolumn{3}{|c|}{ Plutonium-241 } & \multicolumn{3}{|c|}{ Strontium-90 } & \multicolumn{3}{|c|}{ Uranium-233/234 } & \multicolumn{3}{|c|}{ Uranium-235 } & \multicolumn{3}{|c|}{ Uranium-238 } \\
\hline & & & & $\mathrm{pCi} / \mathrm{g}$ & $\mathbf{Q}$ & MDA & $\mathrm{pCi} / \mathrm{g}$ & $\mathbf{Q}$ & MDA & $\mathrm{pCi} / \mathrm{g}$ & $\mathbf{Q}$ & MDA & $\lg$ & $\mathbf{Q}$ & MDA & $\mathrm{Ci} / \mathrm{g}$ & $\mathbf{Q}$ & MDA \\
\hline Northeast corner & 108 & $\mathrm{~J} 13 \mathrm{H} 52$ & $9 / 25 / 06$ & 1.55 & $\bar{U}$ & 20 & 7.72 & & 0.22 & 2.27 & & 0.16 & 0.101 & $\bar{U}$ & 0.19 & 2.23 & & 0.16 \\
\hline Southeast corner & 108 & J13H53 & $9 / 25 / 06$ & 0.682 & $\bar{U}$ & 18 & 3.17 & & 0.24 & 5.47 & & 0.20 & 0.360 & & 0.20 & 6.15 & & 0.16 \\
\hline Northwest corner & 108 & $\mathrm{~J} 13 \mathrm{H} 54$ & $9 / 25 / 06$ & 3.55 & $\mathrm{U}$ & 18 & 0.070 & & 0.23 & 0.848 & & 0.16 & 0 & $\bar{U}$ & 0.20 & 0.997 & & 0.16 \\
\hline Southwest corner & 108 & J13H55 & $9 / 25 / 06$ & -20.6 & $\bar{U}$ & 49 & 0.195 & $\bar{U}$ & 0.22 & 1.64 & & 0.17 & 0.055 & $\bar{U}$ & 0.21 & 1.53 & & 0.17 \\
\hline
\end{tabular}


Table B-8. FIDLER "Hot Spot” Sample Results.

\begin{tabular}{|c|c|c|c|c|c|c|c|c|c|c|c|c|c|c|c|c|c|c|c|c|}
\hline \multirow{2}{*}{ Description } & \multirow{2}{*}{$\begin{array}{l}\text { Sample } \\
\text { Number }\end{array}$} & \multirow{2}{*}{$\begin{array}{c}\text { Sample } \\
\text { Date }\end{array}$} & \multicolumn{3}{|c|}{ Americium-241 } & \multicolumn{3}{|c|}{ Cesium-137 } & \multicolumn{3}{|c|}{ Cobalt-60 } & \multicolumn{3}{|c|}{ Europium-152 } & \multicolumn{3}{|c|}{ Europium-154 } & \multicolumn{3}{|c|}{ Europium-155 } \\
\hline & & & $\mathrm{pCi} / \mathrm{g}$ & $\bar{Q}$ & MDA & $\mathrm{pCi} / \mathrm{g}$ & $\mathbf{Q}$ & MDA & $\mathrm{pCi} / \mathrm{g}$ & $\mathbf{Q}$ & MDA & $\mathrm{pCi} / \mathrm{g}$ & $\mathbf{Q}$ & MDA & $\mathrm{pCi} / \mathrm{g}$ & $\mathbf{Q}$ & MDA & $\mathrm{pCi} / \mathrm{g}$ & $\mathbf{Q}$ & MDA \\
\hline Soil & J135M5 & $8 / 24 / 06$ & $\overline{0}$ & $\bar{U}$ & 1.1 & 2640 & & 5.4 & 2.0 & $\bar{U}$ & 2.0 & 15 & $\bar{U}$ & 15 & 6.4 & $\bar{U}$ & 6.4 & 6.4 & $U$ & 6.4 \\
\hline Soil & J135M6 & $8 / 24 / 06$ & -0.270 & $U$ & 2.1 & 0.79 & $\bar{U}$ & 0.79 & 0.74 & $\mathrm{U}$ & 0.74 & 1.6 & $\bar{U}$ & 1.6 & 2.0 & $\bar{U}$ & 2.0 & 1.9 & $\mathrm{U}$ & 1.9 \\
\hline Soil & J135M7 & $8 / 24 / 06$ & 18.3 & & 0.58 & 12.7 & & 1.7 & 1.9 & $U$ & 1.9 & 3.2 & $\bar{U}$ & 3.2 & 5.4 & $\mathrm{U}$ & 5.4 & 2.7 & $\mathrm{U}$ & 2.7 \\
\hline Debris & J135M8 & $8 / 24 / 06$ & -2.72 & U & 10.0 & 3.0 & $\mathrm{U}$ & 3.0 & 1.7 & $\bar{U}$ & 1.7 & 6.4 & $\bar{U}$ & 6.4 & 5.2 & $\mathrm{U}$ & 5.2 & 120 & $\mathrm{U}$ & 120 \\
\hline Soil & J135M9 & $8 / 24 / 06$ & 1,540 & & 11 & 41.0 & & 0.55 & 0.51 & $\bar{U}$ & 0.51 & 1.3 & $\bar{U}$ & 1.3 & 1.5 & $\mathrm{U}$ & 1.5 & 1.4 & $\mathrm{U}$ & 1.4 \\
\hline Soil & J135NO & $8 / 24 / 06$ & 172 & & 1.6 & 1.7 & $\mathrm{U}$ & 1.7 & 2.0 & $\bar{U}$ & 2.0 & 3.5 & $\bar{U}$ & 3.5 & 6.2 & $\mathrm{U}$ & 6.2 & 2.7 & $\mathrm{U}$ & 2.7 \\
\hline Soil & $\mathrm{N} 1$ & $8 / 24 / 06$ & 0.343 & & 0.20 & 33.4 & & 1.2 & 0.56 & $\mathrm{U}$ & 0.56 & 2.2 & $\mathrm{U}$ & 2.2 & 2.5 & U & 2.5 & 1.4 & $\mathrm{U}$ & 1.4 \\
\hline Soil & J135N2 & $8 / 24 / 06$ & 0.122 & $\bar{U}$ & 0.23 & 4,530 & & $\overline{11}$ & 4.2 & $\bar{U}$ & 4.2 & 36 & $\bar{U}$ & 36 & 13 & $\mathrm{U}$ & 13 & 20 & $U$ & 20 \\
\hline Soil & J135N3 & $8 / 24 / 06$ & 0.086 & $\bar{U}$ & 0.33 & 38.1 & & 2.1 & 2.5 & $U$ & 2.5 & 4.2 & $U$ & 4.2 & 6.5 & $U$ & 6.5 & 3.3 & $U$ & 3.3 \\
\hline
\end{tabular}

\begin{tabular}{|c|c|c|c|c|c|c|c|c|c|c|c|c|c|c|c|c|c|c|c|c|}
\hline \multirow{2}{*}{ Description } & \multirow{2}{*}{$\begin{array}{l}\text { Sample } \\
\text { Number }\end{array}$} & \multirow{2}{*}{$\begin{array}{c}\text { Sample } \\
\text { Date }\end{array}$} & \multicolumn{3}{|c|}{ Plutonium-238 } & \multicolumn{3}{|c|}{ Plutonium-239/240 } & \multicolumn{3}{|c|}{ Plutonium-241 } & \multicolumn{3}{|c|}{ Uranium-233/234 } & \multicolumn{3}{|c|}{ Uranium-235 } & \multicolumn{3}{|c|}{ Uranium-238 } \\
\hline & & & $\mathrm{pCi} / \mathrm{g}$ & $\mathbf{Q}$ & MDA & $\mathrm{pCi} / \mathrm{g}$ & $\mathbf{Q}$ & MDA & $\overline{\mathrm{pCi} / \mathrm{g}}$ & $Q$ & MDA & $\overline{\mathrm{pCi} / \mathrm{g}}$ & $\mathbf{Q}$ & MDA & $\overline{\mathrm{pCi} / \mathrm{g}}$ & $\mathbf{Q}$ & MDA & $\mathrm{pCi} / \mathrm{g}$ & $\mathbf{Q}$ & MDA \\
\hline Soil & J135M5 & $8 / 24 / 06$ & 9.1 & $\mathrm{U}$ & 9.1 & 1.28 & & 0.98 & -1.50 & $\mathrm{U}$ & 47 & 146 & & 0.97 & 7.51 & & 0.4 & 146 & & 0.89 \\
\hline Soil & J135M6 & $8 / 24 / 06$ & -0.274 & $U$ & 2.1 & 0.822 & $\bar{U}$ & 2.1 & -11.9 & $U$ & 100 & 216 & & 1.6 & 11.8 & & 0.72 & 211 & & 1.5 \\
\hline Soil & J135M7 & $8 / 24 / 06$ & 1.66 & & 0.79 & 150 & & 0.79 & 38.1 & $\bar{U}$ & 46 & 53.2 & & 0.56 & 3.58 & & 0.29 & 52.9 & & 0.53 \\
\hline Debris & $\mathrm{J} 135 \mathrm{M} 8$ & $8 / 24 / 06$ & 3.98 & $U$ & 19 & 5.96 & $\bar{U}$ & 15 & 108 & $\mathrm{U}$ & 1,000 & 3,050 & & 20 & 226 & & 6.7 & 3,130 & & 19 \\
\hline Soil & J135M9 & $8 / 24 / 06$ & 56.7 & & 18 & 4,070 & & 14 & 2,070 & $\mathrm{U}$ & 890 & 11.6 & & 5.2 & 2.48 & $\mathrm{U}$ & 6.3 & 5.46 & & 5.2 \\
\hline Soil & J135NO & $8 / 24 / 06$ & 2.63 & & 1.5 & 99.5 & & 1.5 & 56.5 & $\mathrm{U}$ & 82 & 7.49 & & 0.31 & 0.448 & & 0.38 & 7.77 & & 0.31 \\
\hline Soil & $J 135 N 1$ & $8 / 24 / 06$ & 0.054 & $\mathrm{U}$ & 0.41 & 0.862 & & 0.41 & 7.80 & $\mathrm{U}$ & 24 & 3.29 & & 0.044 & 0.182 & & 0.027 & 3.12 & & 0.042 \\
\hline Soil & J135N2 & $8 / 24 / 06$ & 0 & $U$ & 0.31 & 0.080 & $\bar{U}$ & 0.31 & 4.21 & $\mathrm{U}$ & 23 & 0.685 & & 0.030 & 0.069 & & 0.025 & 0.590 & & 0.026 \\
\hline Soil & J135N3 & $8 / 24 / 06$ & 0 & $\bar{U}$ & 0.34 & 0.843 & & 0.27 & -1.84 & $\bar{U}$ & 24 & 0.956 & & 0.033 & 0.051 & & 0.028 & 1.08 & & 0.023 \\
\hline
\end{tabular}


CVP-2006-00010

Rev. 0

B-22 
CVP-2006-00010

Rev. 0

\section{APPENDIX C}

\section{DATA QUALITY ASSESSMENT}

C-i 
CVP-2006-00010

Rev. 0

C-ii 


\section{C1.0 DATA QUALITY ASSESSMENT FOR THE 618-2 SOLID WASTE BURIAL GROUND}

\section{C1.1 OVERVIEW}

The data quality assessment (DQA) completes the data life cycle (i.e., planning, implementation, and assessment) that was initiated by the data quality objectives process. The DQA includes a review of the field logbook information (WCH 2006b) to verify sample location, date, and time. It also involves a scientific and statistical evaluation of the data to determine if they are of the right type, quality, and quantity to support their intended use for closeout decisions (EPA 2000).

This DQA was performed in accordance with data quality objectives found in the 300 Area Remedial Action Sampling and Analysis Plan (SAP) (DOE-RL 2004a). The DQA is based on the guidelines presented in Guidance for Data Quality Assessment (EPA 2000). Statistical tests used in this DQA were performed as specified in the SAP and the Remedial Design Report/Remedial Action Work Plan for the 300 Area (RDR/RAWP) (DOE-RL 2004b).

Prior to performing statistical tests, the field logbook (WCH 2006b), the Closeout Plan for the 618-2 Burial Ground (WCH 2006a), and the sample analytical data were evaluated. A portion of the cleanup verification sample analytical data are validated for compliance requirements (DOE-RL 2004a). An evaluation is performed to determine if the laboratory carried out all steps required by the SAP and the laboratory contract governing the conduct of analysis and reporting of the data. Third-party data validation, in accordance with validation procedures specified in Data Validation Procedure for Chemical Analysis (BHI 2000a) and in Data Validation Procedure for Radiochemical Analysis (BHI 2000b), is performed as part of data evaluation. After validation and data evaluation, the appropriate statistical analyses are performed on the analytical data (Appendix D) to determine statistical values, as appropriate, for each contaminant. The cleanup verification sample analytical data are stored in the Environmental Restoration (ENRE) project-specific database prior to being submitted for inclusion in the Hanford Environmental Information System (HEIS) database and are also summarized in Appendix B.

\section{C1.2 LABORATORY QUALITY MEASURES}

All verification samples are subject to laboratory-specific quality assurance (QA) requirements, including instrument procurement, maintenance, calibration, and operation. Additional laboratory quality control (QC) checks are performed, as appropriate, for the analytical method at a rate of 1 per sample delivery group (SDG), or 1 per 20 samples, whichever is more frequent. Laboratory internal QC checks include the following: 
- Laboratory Contamination. Each analytical batch contains a laboratory (method) blank (material of similar composition as the samples with known/minimal concentrations of the analytes of interest) carried through the complete analytical process. The method blank is used to evaluate samples for false-positive results due to contamination at the laboratory.

- Analytical Accuracy. For most analyses, a known quantity of representative analytes of interest are added to a separate aliquot of a sample from the analytical batch, known as the matrix spike/matrix spike duplicate (MS/MSD). The recovery percentages of the added MS are used to evaluate analytical accuracy. For analyses not amenable to MS techniques (e.g., gamma energy analysis) or where analytical recovery is corrected via internal standards (e.g., alpha spectral analyses), accuracy is evaluated from recovery of the QC reference sample (i.e., laboratory control spike [LCS] or blank spike sample).

- Analytical Precision. Separate aliquots removed from the same sample container are analyzed for each analytical batch, and referred to as the laboratory duplicate or replicate. The replicate sample results (evaluated by relative percent differences [RPDs]) are used to assess the analytical precision achieved at the laboratory. However, natural heterogeneities often found in soil matrix samples also play a significant part in the RPD result.

- QC Reference Samples. A QC reference sample is prepared from an independent standard at a concentration other than that used for calibration, but within the calibration range. Reference samples provide an independent check on analytical technique and methodology.

Laboratories are also subject to periodic and random assessments of the laboratory performance, systems, and overall program. These assessments are performed by the Washington Closure Hanford QA group to ensure that the laboratories are performing within laboratory contract requirements.

\section{C1.3 DATA VALIDATION}

After sampling was completed, all of the fixed laboratory data from SDG K0545 were submitted for third-party validation to Level $\mathrm{C}$. Level $\mathrm{C}$ validation procedures are specified in Data Validation Procedure for Radiochemical Analysis (BHI 2000b) and Data Validation Procedure for Chemical Analysis (BHI 2000a).

Level C validation procedures were used to review and qualify the data for the following parameters:

- Sample holding times

- Method blanks

- MS/MSD recovery

- Surrogate recovery 
- Sample replicates (duplicates)

- Laboratory control sample results

- Data package completeness

- Achievement of required detection limits (RDLs) or contract required quantitation limits.

Data qualified as rejected (i.e., "R" flagged) indicate that the associated analytical result is tainted by a major deficiency in the quality of the data. Rejected data are unsuitable for decision-making purposes. Data qualified as estimated (i.e., "J" flagged) indicate that the data is estimated, but may be used for decision- making purposes. Data qualified as undetected (i.e., "U" flagged) indicate the analyte was analyzed for but not detected. For nonradionuclides, nondetected results are reported at the practical quantitation limit (PQL). For radionuclides, nondetected results are reported at the actual value obtained from analysis (positive or negative but less than the minimum detected activity [MDA]), except for limited analyses where no value can be calculated and the analytes are reported nondetected at the MDA. All other validated results are considered accurate within the standard errors associated with the methods.

The adequacy of laboratory $\mathrm{QA} / \mathrm{QC}$ was evaluated for precision, accuracy, completeness, and RDLs pursuant to the SAP (DOE-RL 2004a). The organization performing the data validation reported that, of the data given formal validation, the laboratory met the standards for performance for precision $( \pm 30 \%)$, accuracy $( \pm 30 \%)$, and completeness (>90\%).

\section{SDG K0545}

This data package contains 17 samples (J13D59 through J13D75) analyzed by alpha spectroscopy, tritium analysis, nickel-63 analysis, plutonium-241 analysis, total uranium analysis, total strontium analysis, gamma spectroscopy, and by inductively coupled plasma (ICP) metals analysis. The 17 samples were collected from the 618-2 overburden and the staging pile/decontamination pad footprint. Sample J13D74 (A2) is the main sample and sample J13D59 is the corresponding field duplicate. SDG K0545 was evaluated through a formal third-party validation process. A summary of the validation follows:

Radionuclides. No major deficiencies were found in the SDG K0545 radiological data. Minor deficiencies are as follows:

The radiochemical yield in sample J13D61 was outside the QC limits at 19\%. Thirdparty validation qualified all of the plutonium-238 and plutonium-239/240 results in sample J13D61 as estimated with "J" flags. Estimated data are useable for decisionmaking purposes. 
Due to a lack of MS analysis, third-party validation qualified all tritium results in SDG K0545 as estimates with "J" flags. Matrix spikes for some radionuclides are not typically performed by the laboratory. Other accuracy measures, such as blind audit samples, are used to assess laboratory accuracy for these radionuclides. Estimated data are useable for decision- making purposes.

Due to an RPD outside the QC limits (thorium-228, 34\%), third-party validation qualified all of the thorium-228 results in SDG K0545 as estimated with "J " flags. Estimated data are useable for decision- making purposes.

Nonradionuclides. No major or minor deficiencies were found in the SDG K0545 nonradiological data.

Limited, random, or sample matrix-specific influenced batch QC issues such as those identified in the radionuclide data for SDG K0545 are potential issues for any analysis. The number and types seen in SDG K0545 are within expectations for the matrix types and analyses performed. All of the data in SDG K0545 are useable for decision-making purposes.

\section{C1.4 LABORATORY DATA EVALUATION}

The following paragraphs include a data evaluation of the remaining verification sample SDGs (K0547, K0580, K0586, J00098, J00099) for the 618-2 waste site. Comments on the comparability of the samples to the project splits are presented in section $\mathrm{C} 1.5$.

\section{SDG K0547}

This data set comprises 18 field samples (J13DF9, J13DH0 through J13DH3, J13DH9, J13DJ0 through J13DJ9, J13DK0, and J13DK1). These samples are a mixture of shallow zone, deep zone, overburden, overburden footprint, and biased samples. Appendix $D$ provides specific sample locations and analyte lists for samples from the 618-2 waste site. Samples J13DJ7 (shallow zone, A2), J13DJ0 (deep zone, A2) and sample J13DH0 (overburden, A2) are "main" samples in conjunction with corresponding duplicate samples J13DJ8, J13DJ1, and J13DH1, respectively. Sample J13DK1 is the equipment blank (field blank) for the 618-2 waste site. Samples J13DJ5 and J13DH9 are bias samples.

Radionuclides. No major deficiencies were found in the SDG K0547 radiological data. Minor deficiencies are as follows:

- Detected quantities of uranium-233/234 (161 pCi/g) and uranium-238 (165 pCi/g) in sample J13DJ3 caused spectral interference in the analysis of uranium-235. Due to this interference, the MDA for uranium-235 in sample J13DJ3 was elevated to 5.8 $\mathrm{pCi} / \mathrm{g}$. This result is typical of radiological counting methods. The uranium-233/234 
and uranium-238 results are large enough that a minimal increase in the uranium$235 \mathrm{MDA}$ is not a significant problem for this waste site. The data are useable for decision-making purposes.

- The MDAs for plutonium-241 was also elevated in the SDG K0547 sample data. In this case, the elevated MDAs are due to slightly shortened counting times. The resulting MDAs are all well below the RAG values. The data are useable for decision-making purposes.

Nonradionuclides. No major deficiencies were found in the SDG K0547 nonradiological data. Minor deficiencies are as follows:

- In the semivolatile organic analysis, of sample J13DH9, low recoveries were observed in the MSs and some LCSs for 8 analytes. The analytes are 2-methylphenol, 4-methylphenol, nitrobenzene, isophorone, 2,4-dimethylphenol, 1,2,4-trichlorobenzene, 4-chloro-3-methylphenol, and 2-methylnaphthalene. The $\mathrm{MS} / \mathrm{MSD}$ and/or LCS recoveries in question were all in the range of $39 \%$ to $59 \%$. The project has qualified results for these analytes, in sample J13DH9, as estimated and assigned " $\mathrm{J}$ " flags to the data.

- In the ICP metals analysis, the analyte tin was detected in the method blank (MB) and in all of the samples. The MB concentration is greater than the PQL and greater than $1 / 20^{\text {th }}$ of all of the sample concentrations. The project has qualified all of the results for tin in SDG K0547 as estimated with "J" flags. Estimated data are useable for decision- making purposes.

- In the ICP metals analysis, the RPD results for tin and arsenic were above the acceptance criteria at $57.1 \%$ and $51.2 \%$, respectively. Because the duplicate is prepared from actual field sample matrix (soil), the heterogeneities within the matrix contribute to the RPD result. This deficiency does not indicate a problem with the analytical laboratory methodology. The data are useable for decision-making purposes.

\section{SDG K0580}

This data set comprises five field samples (J13DN0 through J13DN4). These are biased samples collected within the 618-2 excavation.

Radionuclides. No major deficiencies were found in the SDG K0580 radiological data. Minor deficiencies are as follows:

- The MDAs for plutonium-241 are elevated in the SDG K0580 data. In this case, the elevated MDAs are due to slightly shortened counting times. The resulting MDAs are all well below the RAG values. The data are useable for decision-making purposes. 
Nonradionuclides. No major or minor deficiencies were found in the SDG K0080 radiological data.

\section{SDG K0086}

This data set comprises two field samples (J13H86 and $\mathrm{J} 13 \mathrm{H} 87)$. These samples are biased within the 618-2 excavation.

Radionuclides. No major deficiencies were found in the SDG K0586 radiological data. Minor deficiencies are as follows:

- The MDAs for plutonium-241 are elevated in the SDG K0586 data. In this case, the elevated MDAs are due to slightly shortened counting times. The resulting MDAs are all well below the RAG values. The data are useable for decision-making purposes.

Nonradionuclides. No major or minor deficiencies were found in the SDG K0086 radiological data.

\section{SDG J00098}

This data set comprises one field sample (J13D76). Sample J13D76 is a project split of sample J13D74.

Radionuclides. No major deficiencies were found in the SDG J00098 radiological data. Minor deficiencies are as follows:

- Insufficient sample matrix was sent to the laboratory to provide for a duplicate sample. The sample (J13D76) was analyzed on two separate detectors to provide a duplicate analysis. This is acceptable. The data are useable for decision-making purposes.

Nonradionuclides. No major deficiencies were found in the SDG J00098 radiological data. Minor deficiencies are as follows:

- The MS/MSD results for cadmium are $59 \%$ and $58 \%$, respectively. The field sample results for cadmium should be considered estimated. Estimated data are useable for decision-making purposes. 


\section{SDG J00099}

This data set comprises three field samples (J13DH4, J13DK2, and J13DK3). Samples J13DH4, J13DK2, and J13DK3 are field splits of samples J13DH0, J13DJ7, and J13DJ0, respectively.

Radionuclides. No major deficiencies were found in the SDG J00099 radiological data. Minor deficiencies are as follows:

- The LCS result for americium-241 was above the acceptance criteria at $131 \%$. This implies a slight high bias in the data. High-biased data are acceptable for decisionmaking purposes.

- The LCS result for radium-226 was below the acceptance criteria at $69 \%$. This implies a low bias in the data. The radium-226 results in SDG J00099 are considered estimated. Estimated data are useable for decision-making purposes.

Nonradionuclides. No major deficiencies were found in the SDG J00098 radiological data. Minor deficiencies are as follows:

- The MS/MSD results for cadmium are $59 \%$ and $58 \%$, respectively. The sample results for cadmium should be considered estimated. Estimated data are useable for decision- making purposes.

- The MS result for silver was below the acceptance criteria at $65 \%$. The MSD, LCS, and RPD results for silver are all within the acceptance criteria range. The silver results may be considered estimated but useable for decision-making purposes.

\section{C1.4.1 MAJOR DEFICIENCIES}

Any data anomaly that causes final data to be qualified as rejected ("R" flagged) is considered a major deficiency. No major deficiencies were found the 618-2 verification data set.

\section{C1.4.2 MINOR DEFICIENCIES}

Sample Holding Times. All of the method-specific holding times were met for all samples in the 618-2 verification data set.

Method Blanks. The method blank is used to evaluate false-positive results in samples due to contamination during handling at the laboratory.

Radionuclides. All of the radionuclide method blank results were within the acceptance criteria. 
CVP-2006-00010

Rev. 0

Nonradionuclides. A minor method blank deficiency was identified in SDG K0547 (see above).

MS/MSDs Recoveries. Recoveries of spiked analytes in the MS/MSD pair(s) are used to evaluate method efficiency and the effect of the sample matrix on the environmental sample results.

Radionuclides. A minor method blank (MB) deficiency was identified in SDG K0547 (see above).

Nonradionuclides. Minor deficiencies in the MS/MSD recoveries are identified in SDGs K0547, J00098, and J00099. Some data are qualified as estimated. Estimated data are useable for decision-making purposes.

RDL Comparison. Reported analytical detection levels for nondetected analytes were compared to the RDLs specified in the SAP (DOE-RL 2004a). When detected results were obtained, evaluation of detection limits was not performed.

Radionuclides. All of the reported contaminant of concern (COC) MDAs are sufficiently low for decision-making purposes. All values meet the site cleanup criteria as demonstrated in the calculation briefs (Appendix D) and discussed in this cleanup verification package.

Nonradionuclides. All of the reported MDLs are sufficiently low for decision-making purposes. All values meet the site cleanup criteria as discussed in this cleanup verification package.

Precision and Accuracy Evaluation. RPD evaluation of the main sample(s) versus the laboratory duplicate(s) are routinely performed by the laboratory, and any deficiencies in the calculations are reported by SDG in section C1.4.0.

\section{C1.5 FIELD QUALITY ASSURANCE/QUALITY CONTROL}

Field QA/QC measures were used to assess potential sources of error and cross contamination of soil samples that could bias results. Field QA/QC samples listed in the field logbook (WCH 2006b) are summarized in Table C-1. The main and QA/QC sample results are presented in Appendix B.

Table C-1. Summary of Field Quality Control Samples.

\begin{tabular}{|l|c|c|c|}
\hline Sample location (designation) & Main Sample & Duplicate Sample & Split Sample \\
\hline Staging pile/decon pad (A2) & $\mathrm{J} 13 \mathrm{D} 74$ & $\mathrm{~J} 13 \mathrm{D} 59$ & $\mathrm{~J} 13 \mathrm{D} 76$ \\
\hline Overburden (A2) & $\mathrm{J} 13 \mathrm{DH} 0$ & $\mathrm{~J} 13 \mathrm{DH} 1$ & $\mathrm{~J} 13 \mathrm{DH} 4$ \\
\hline Shallow zone (A2) & $\mathrm{J} 13 \mathrm{DJ} 7$ & $\mathrm{~J} 13 \mathrm{DJ} 8$ & $\mathrm{~J} 13 \mathrm{DK} 2$ \\
\hline Deep zone (A2) & $\mathrm{J} 13 \mathrm{DJ} 0$ & $\mathrm{~J} 13 \mathrm{DJ} 1$ & $\mathrm{~J} 13 \mathrm{DK} 3$ \\
\hline
\end{tabular}


Field duplicate samples are collected to provide a relative measure of the degree of local heterogeneity in the sampling medium, unlike laboratory duplicates that are used to evaluate precision in the analytical process. The field duplicates are evaluated by computing the RPD of the duplicate samples for each COC. Only analytes with values above five times the detection limits for both the main and duplicate samples are calculated and compared. The 95\% upper confidence limit (UCL) calculation brief in Appendix D provides details on duplicate pair evaluation and RPD calculation. The data are suitable for the intended purpose of cleanup verification.

Split samples are collected to provide a relative measure of the degree of variability in the sampling, sample handling, and analytical techniques used by commercial laboratories. The field main and split samples are evaluated by computing the RPD of the split samples for each $\mathrm{COC}$ to determine the usability of the verification data. The U.S. EPA Contract Laboratory Program duplicate sample comparison methodology, USEPA Contract Laboratory Program National Functional Guidelines for Inorganic Data Review (EPA 1994), is used as an initial test of the data from the splits. Only analytes that had values above five times the contractual RDL for both the main and split sample were compared. The $95 \% \mathrm{UCL}$ calculation briefs in Appendix D provide details on split pair RPD calculation. The acceptance criteria RPD for project-split samples is $\leq 30 \%$ (less than or equal to $30 \%$ ).

\section{Radionuclides.}

- The RPDs calculated for the deep zone project split sample (J13DK3) analytes americium-241, plutonium-239/240, uranium-233/234, uranium-238, and uranium (metal) are above the acceptance criteria (30\%) at $77 \%, 57 \%, 67 \%, 66 \%$, and $86 \%$, respectively. Elevated RPDs, such as these, in the analysis of environmental soil samples, are largely attributed to heterogeneities in the soil matrix and only in small part attributed to precision and accuracy issues at the laboratory. The data are useable for decision-making purposes.

\section{Nonradionuclides.}

- The RPD calculated for the staging pile/decontamination pad project split sample (J13D76) analyte chromium is above the acceptance criteria (30\%) at 54\%.

- The RPD calculated for the deep zone project split sample (J13DK3) analyte uranium (metal) is above the acceptance criteria (30\%) at $86 \%$.

Elevated RPDs, such as these, in the analysis of environmental soil samples, are largely attributed to heterogeneities in the soil matrix and only in small part attributed to precision and accuracy issues at the laboratory. The data are useable for decisionmaking purposes.

RPDs for the remaining nonradionuclide analytes were either within the acceptance criteria or were not calculated because an evaluation of the data shows the analytes 
were not detected in both the main and duplicate (or main and split) sample at more than 5 times the target detection limit. RPDs of analytes detected at low concentrations (less than five times the detection limit) are not considered indicative of the analytical system performance. The data are useable for decision-making purposes.

A visual inspection of all of the data is also performed by the data reviewer. No major deficiencies were noted. No additional minor deficiencies were noted. The data are useable for decision-making purposes.

\section{C1.6 SUITABILITY OF DATA}

The DQA for the 618-2 waste site determined that the data are of the right type, quality, and quantity to support site cleanup verification decisions within specified error tolerances. The DQA verified that the sample design was sufficient for the purpose of clean site verification.

\section{C2.0 REFERENCES}

BHI, 2000a, Data Validation Procedure for Chemical Analysis, BHI-01435, Rev. 0, Bechtel Hanford, Inc., Richland, Washington.

BHI, 2000b, Data Validation Procedure for Radiochemical Analysis, BHI-01433, Rev. 0, Bechtel Hanford, Inc., Richland, Washington.

DOE-RL, 2004a, 300 Area Remedial Action Sampling and Analysis Plan, DOE/RL-2001-48, Rev. 1, U.S. Department of Energy, Richland Operations Office, Richland, Washington.

DOE-RL, 2004b, Remedial Design Report/Remedial Action Work Plan for the 300 Area, DOE/RL-2001-47, Rev. 1, U.S. Department of Energy, Richland Operations Office, Richland, Washington.

EPA, 1994, USEPA Contract Laboratory Program National Functional Guidelines for Inorganic Data Review, EPA 540/R-94/013, U.S. Environmental Protection Agency, Washington, D.C.

EPA, 2000, Guidance for Data Quality Assessment, EPA QA/G-9, QA00 Update, U.S. Environmental Protection Agency, Office of Environmental Information, Washington, D.C.

WCH, 2006a, Closeout Plan for the 618-2 Burial Ground, CCN 129577, Washington Closure Hanford, Richland, Washington.

WCH, 2006b, Remedial Sampling, Logbook EFL-1395-12, Washington Closure Hanford, Richland, Washington. 
APPENDIX D

CALCULATION BRIEF EXCERPTS 
CVP-2006-00010

Rev. 0

\section{DISCLAIMER FOR CALCULATIONS}

The calculations that are provided in the following appendix have been generated to document compliance with established cleanup levels. These calculations should be used in conjunction with other relevant documents in the administrative record. 


\section{CALCULATION BRIEFS}

The following calculation briefs have been prepared in accordance with ENG-1, Engineering Services, ENG-1-4.5, "Project Calculations," Washington Closure Hanford, Richland, Washington.

WCH, 2006, Cleanup Verification 95\% UCL Calculations for 618-2 Shallow Zone/Deep Zone/Overburden and 618-2, 618-3, and 618-8 Staging Pile and Decon Pad Footprint, Calculation No. 0600X-CA-V0060, Rev. 2, Washington Closure Hanford, Richland, Washington.

WCH, 2006, 618-2 Shallow, Deep Zone, Overburden/Stockpile Area Sampling Plan, 0300X-CA-V0077, Rev. 0, Washington Closure Hanford, Richland, Washington.

WCH, 2006, 618-2 Burial Ground Cleanup Verification RESRAD Calculation Brief, Calculation No. 0300X-CA-V0080, Rev. 0, Washington Closure Hanford, Richland, Washington.

NOTE: The calculation briefs referenced in this appendix are kept in the active Washington Closure Hanford project files and are available upon request. When the project is completed, the files will be stored in a U.S. Department of Energy, Richland Operations Office repository. 
CVP-2006-00010

Rev. 0

D-2 
CVP-2006-00010

Rev. 0

\section{CALCULATION COVER SHEET}

\begin{tabular}{lll}
$\begin{array}{l}\text { Project Title } \\
\text { Area } \\
\text { Discipline } \\
\text { Subject }\end{array}$ & 300 Area Field Remediation & Job No. \\
\cline { 2 - 2 } & $\frac{600}{\text { Environmental }}$ & \\
\hline Computer Program & Cleanup Verification 95\% UCL Calculations for 618-2 Shallow Zone/Deep Zone/Overburden, \\
\hline and 618-2,618-3,618-8 Staging Pile and Decon Pad Footprint \\
\hline
\end{tabular}

The attached calculations have been generated to document compliance with established cleanup levels. These calculations should be used in conjunction with other relevant documents in the administrative record.

Committed Calculation $\square \quad$ Preliminary $\square \quad$ Superseded $\square \quad$ Voided $\square$

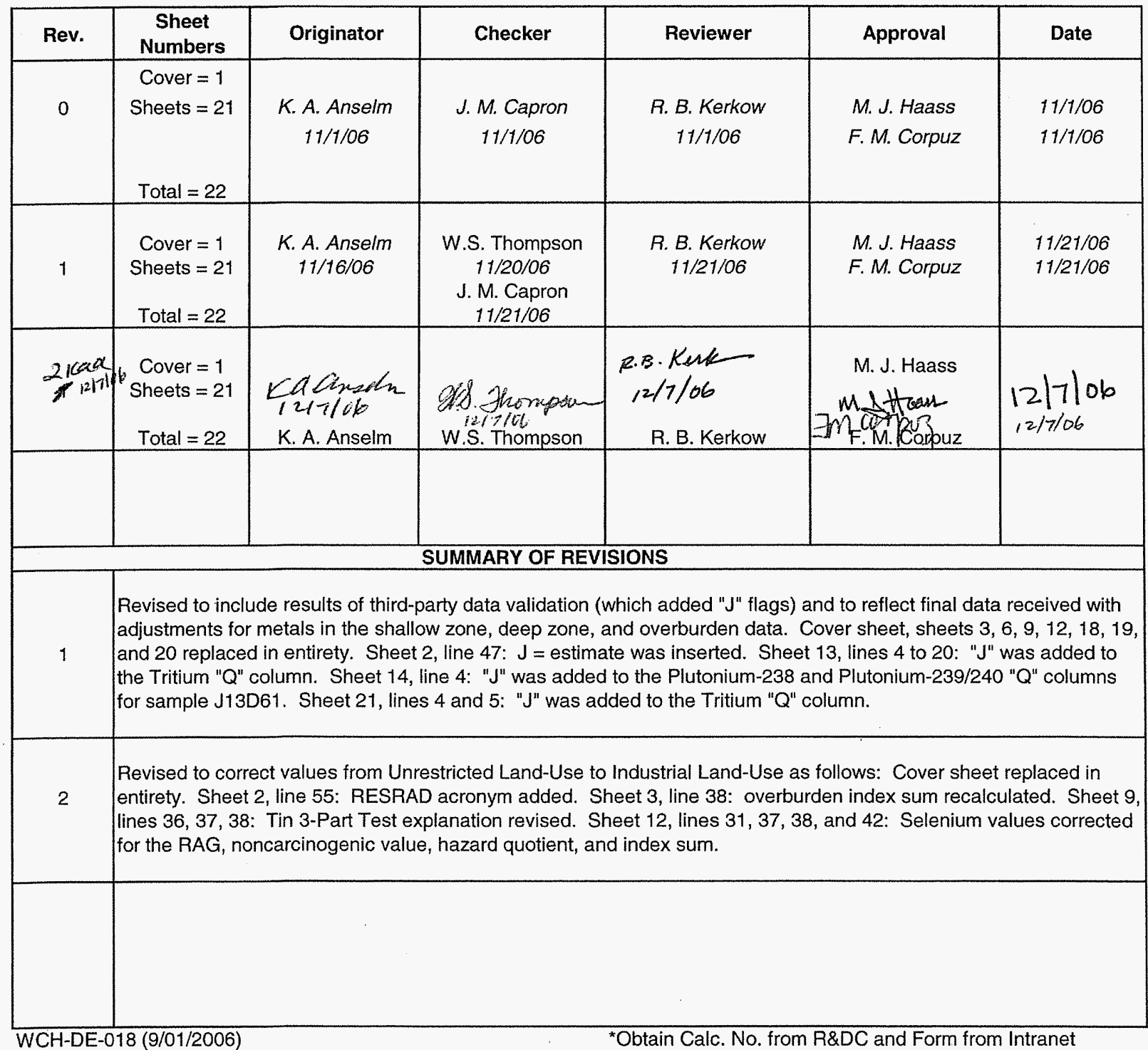


Washington Closure Hanford

Originator K. A. Anselm $\quad \mathrm{CCW}$ Project 300 Area Field Remediation Subject Cleanup Verification $95 \%$ UCL for 618-2 Shallow
Date 11/01/06 Job No. 14655

\section{CALCULATION SHEET}

or 618-2 Shallow Z
Calc. No. 0600X-CA-V0060 Checked J.M. Capron L/2x Checked J.M.

Summary

1 Purpose:

2 This document contains calculations based on analytical results from data collected from the 618-2 shallow zone, deep zone, and overburden piles, as well as a staging pile and decon pad footprint used for staging excavation material from the 618-2, 618-3, and 618-8 Burial Grounds. Other calculations to support cleanup of 618-3 and 618-8 Burial Grounds are included in cleanup verification packages (CVPs) for those sites. The calculations in this document include (1) calculation of the $95 \%$ upper confidence limit (UCL) to evaluate compliance with cleanup standards, (2) calculation of the carcinogenic risk for applicable nonradionuclide analytes, (3) performance of the Washington Administrative Code (WAC) 173-340 (Model Toxics Control Act [MTCA]) 3-part test, as required, and (4) calculation of the relative percent difference (RPD) for each contaminant of concern (COC)/contaminant of potential concern (COPC).

Table of Contents:

Sheets 1 to 3 - Summary

Sheets 4 to 6 - 618-2 Shallow Zone Verification

Sheets 7 to 9 - 618-2 Deep Zone Verification

Sheets 10 to $12-618-2$ Overburden Verification

Sheets 13 to 16 - 618-2, 618-3, and 618-8 Staging Pile/Decon Pad Footprint Verification

Sheet 17 - MTCAStat Results

Sheets 18 to 21 - Split-Duplicate Analyses

Given/References:

1) Sample Results

2) Background values and remedial action goals (RAGs) are from DOE-RL (2001 and 2004b), and Ecology (1994).

3) DOE-RL, 2001, Hanford Site Background: Part 1, Soil Background for Nonradioactive Analytes, DOE/RL-92-24, Rev. 4, U.S. Department of Energy, Richland Operations Office, Richland, Washington.

4) DOE-RL, 2004a, 300 Area Remedial Action Sampling and Analysis Plan, (SAP) DOE/RL 2001-48, Rev. 1, U.S. Department of Energy, Richland Operations Office, Richland, Washington.

5) DOE-RL, 2004b, Remedial Design Report/Remedial Action Work Plan for the 300 Area (RDR/RAWP), DOE/RL-2001-47, Rev. 1, U.S. Department of Energy, Richland Operations Office, Richland, Washington.

6) Ecology, 1992, Statistical Guidance for Ecology Site Managers, Publication \#92-54, Washington Department of Ecology, Olympia, Washington.

7) Ecology, 1993, Statistical Guidance for Ecology Site Managers, Supplement S-6, Analyzing Site or Background Data with Below-detection Limit or Below-PQL Values (Censored Data Sets), Publication \#92-54, Washington Department of Ecology, Olympia, Washington.

8) Ecology, 1994, Natural Background Soil Metals Concentrations in Washington State, Publication No. 94-115, Washington State Department of Ecology, Olympia, Washington.

9) EPA, 1994, USEPA Contract Laboratory Program National Functional Guidelines for Inorganic Data Review, EPA 540/R-94/013, U.S. Environmental Protection Agency, Washington, D.C.

10) WAC 173-340, 1996, "Model Toxic Control Act - Cleanup," Washington Administrative Code.

Solution:

Calculation methodology is described in Ecology Pub. \#92-54 (Ecology 1992, 1993), below, and in the RDR/RAWP (DOE-RL 2004b). Use data from the attached worksheets to calculate the 95\% UCL, hazard quotients, excess carcinogenic risk, perform the WAC $173-340$ 3-part test for nonradionuclides, and calculate the RPD for each COC/COPC in the primary-duplicate and primary-split sample pairs.

Calculation Description:

The subject calculations were performed on data from soil verification samples from the 618-2 waste site, and the 618-2, 618-3, and 618-8 staging pile/decon pad footprint. The data were entered into an EXCEL 2003 spreadsheet and calculations performed by utilizing the built-in spreadsheet functions and/or creating formulae within the cells. The statistical evaluation of data for use in accordance with the RDR/RAWP (DOE-RL 2004b) Methodology:

For nonradioactive analytes with $<50 \%$ of the data below detection limits and all radionuclide analytes, the statistical value calculated to evaluate the effectiveness of cleanup is the $95 \%$ UCL. For nonradioactive analytes with $>50 \%$ of the data below detection limits, the maximum value for the data set is used instead of the $95 \%$ UCL. All nonradionuclide data reported as being below detection limits are set to $1 / 2$ the detection limit value for calculation of the statistics (Ecology 1993). For radionuclide data, calculation of the statistics was done on the reported value. In cases where 8 the laboratory does not report a value below the minimum detectable activity (MDA), half of the MDA is used in the calculation. For the statistical 9 evaluation of primary-duplicate sample pairs, the samples are averaged before being included in the data set, after adjustments for censored data as described above. 
CVP-2006-00010

Rev. 0

\section{Washington Closure Hanford}

Originator K. A. Anselm $/ C \angle C L$

Project 300 Area Field Remediation

\section{CALCULATION SHEET}

$\begin{array}{ll}\text { Date } \frac{12 / 07 / 06}{14655} & \text { Calc. No. } 0600 \text { X-CA-V0060 } \\ \text { Checked W.S.Thompson } 3 / 48\end{array}$ or 618-2 Shallow Zone/Deep Zone/Overburden, $\begin{aligned} \text { Rev. No. } & \frac{2}{12 / 07 / 06} \\ \text { Date } & \frac{106}{21}\end{aligned}$

Summary (continued)

1 Methodology (continued):

2 For nonradionuclides, the WAC 173-340 statistical guidance suggests that a test for distributional form be performed on the data and the $95 \%$ UCL calculated on the appropriate distribution using Ecology software. For nonradionuclide small data sets $(n<10)$ and all radionuclide data sets, the calculations are performed assuming nonparametric distribution, so no tests for distribution are performed. For nonradionuclide data sets of ten or greater, as for the subject site, distributional testing and calculation of the $95 \%$ UCL is done using Ecology's MTCAStat software (Ecology 1993). Due to differences in addressing censored data between the RDR/RAWP (DOE-RL 2004b) and MTCAStat coding and due to a limitation in the MTCAStat coding (no direct capability to address variable quantitation limits within a data set), substitutions for censored data are performed before software input and the resulting input sef treated as uncensored.

The hazard quotient (for shallow zone nonradionuclide COCs/COPCs) is determined by dividing the statistical value (derived in this calculation) by the WAC 173-340 noncarcinogenic cleanup limit. The excess nonradionuclide carcinogenic tisk is determined by dividing the statistical value by the WAC 173-340 carcinogenic cleanup limit and then multiplying by $10^{-6}$.

The WAC 173-340 3-part test is performed for nonradionuclide analytes only and determines if:

1) the $95 \%$ UCL value exceeds the most stringent cleanup limit for each nonradionuclide COC/COPC,

2) greater than $10 \%$ of the raw data exceed the most stringent cleanup limit for each nonradionuclide COC/COPC,

3) the maximum value of the raw data set exceeds two times the most stringent cleanup limit for each nonradionuclide $\mathrm{COC} / \mathrm{COPC}$

The RPD is calculated when both the primary value and either the duplicate or split values are above detection limits and are greater than 5 times the target detection limit (TDL). The TDL is a laboratory detection limit predetermined for each analytical method as listed in Table $2-1$ of the SAP (DOE-RL 2004a). The RPD calculations use the following formula: RPD $=[M-S \mid /(M+S) / 2)]^{*} 100$

$$
\text { where, } M=\text { Main Sample Value } \quad S=\text { Split (or duplicate) Sample Value }
$$

For quality assurance/quality control (QA/QC) split and duplicate RPD calculations, a value less than $+/-30 \%$ indicates the data compare favorably. For regulatory splits, a threshold of $35 \%$ is used (EPA 1994). If the RPD is greater than $30 \%$ (or $35 \%$ for regulatory split data), further investigation regarding the usability of the data is performed. Additional discussion as necessary is provided in the data quality assessment section of the CVP.

If regulator split comparison is required, an additional parameter is evaluated. A control limit of $+/-2$ times the TDL shall be used if either the main or regulator split value is less than 5 times the TDL and above detection. In the case where only one result is greater than 5 times the TDL and the other is below, the $+1-2$ times the TDL criteria applies. Therefore, the following calculation is performed as part of the evaluation for these two cases involving regulator split data: difference = main - regulator split. If the difference is greater than $+1-2$ times the TDL, then further investigation regarding the usability of the data is performed and presented in the applicable CVP data quality assessment section. No regulatory split samples were collected for this site.

4 Abbreviations/Acronyms:

$42 B G$ = background

$43 \mathrm{COC}=$ contaminant of concern

$44 \mathrm{COPC}=$ contaminant of potential concern

$45 \mathrm{CVP}=$ cleanup verification package

$46 \mathrm{GW}=$ groundwater

$47 \mathrm{~J}=$ estimate

$48 \mathrm{MDA}=$ minimum detectable activity

49 MTCA = Model Toxics Control Act

$50 \mathrm{NA}=$ not applicable

$51 \mathrm{PQL}=$ practical quantitation limit

$52 Q=$ qualifier

53 QA/QC = quality assurance/quality control

$54 R A G=$ remedial action goal

55 RESRAD = RESidual RADioactivity (dose model)

$56 \mathrm{RDR} / \mathrm{RAWP}=$ remedial design report/remedial action work plan

$57 \mathrm{RPD}=$ relative percent difference

58 SAP = sampling and analysis plan

$59 \mathrm{TDL}=$ target detection limit

$60 \mathrm{U}=$ less than MDA.PQL

$61 \mathrm{UCL}=$ upper confidence limit

$62 \mathrm{WAC}=$ Washington Administrative Code 
CVP-2006-00010

Rev. 0

Washington Closure Hanford

Project 300 Area Field Remediation
Originator K. A. Anselm $\mathrm{C} C \mathrm{a} a$

\section{CALCULATION SHEET}

Date 12/07/06 Calc. No. 0600X-CA-V0060 Rev. No. 2 Job No. 14655 Checked W.S. Thompson Pr/fhomptr Date 12/07/06 and 618-2, 618-3, 618-8 Staging Pile and Decon Pad Footprint

Summary (continued)

1 Results:

2 The results presented in the summary tables that follow are for use in the 618-2 CVP.

3

$4 \quad$ Results Summary

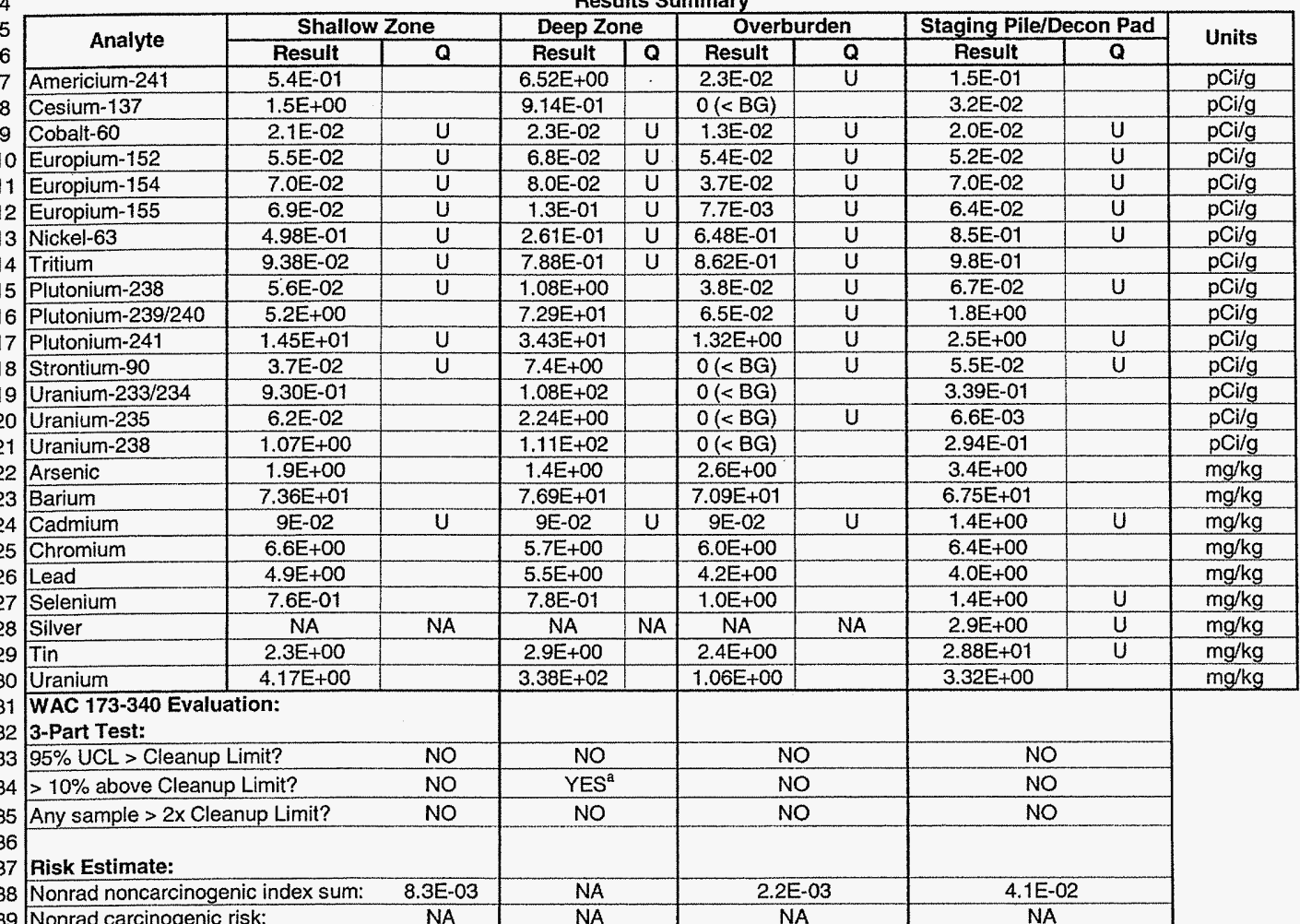

39 Nonrad carcinogenic risk:

NA NA 1 NA

NA

40 "Because of the "yes" answer to the WAC 173-340 3-part test, additional site-specific evaluation will be performed and included in the CVP.

41

42 Relative Percent Difference Results and QAQC Analysis*

\begin{tabular}{|c|c|c|c|c|c|c|}
\hline \multirow[b]{2}{*}{ Analyte } & \multicolumn{2}{|c|}{ Deep Zone } & \multicolumn{2}{|c|}{ Overburden } & \multicolumn{2}{|c|}{ Staging Pile/Decon Pad } \\
\hline & $\begin{array}{l}\text { Duplicate } \\
\text { Analysis }{ }^{* *}\end{array}$ & $\begin{array}{c}\text { Split } \\
\text { Analysis }^{\star \star} \\
\end{array}$ & $\begin{array}{l}\text { Duplicate } \\
\text { Analysis }\end{array}$ & Split Analysis ${ }^{\star \star}$ & \begin{tabular}{c|} 
Duplicate \\
Analysis
\end{tabular} & $\begin{array}{c}\text { Split } \\
\text { Analysis }^{\star *}\end{array}$ \\
\hline Americium-241 & $15 \%$ & $77 \%$ & & & & \\
\hline Plutonium-239/240 & $13 \%$ & $57 \%$ & & & & \\
\hline Strontium-90 & $21 \%$ & $23 \%$ & & & & \\
\hline Uranium-233/234 & $13 \%$ & $67 \%$ & & & & \\
\hline Uranium-238 & $17 \%$ & $66 \%$ & & & & \\
\hline Uranium & $4 \%$ & $86 \%$ & & & & \\
\hline Chromium & & & $22 \%$ & $28 \%$ & $23 \%$ & $54 \%$ \\
\hline
\end{tabular}

53 *RPD listed where result produced, based on criteria. If RPD not required, no value is listed.

54 **The significance of the reported RPD values, including values greater than $30 \%$, is addressed in the CVP data quality assessment section.

55

57

D-6 


\section{Washington Closure Hanford}

Originator K. A. Anselm ICan

(c)

and 018 - 618 - Shallow Zone/Deep Zone/Overburden
Date $\frac{11 / 01 / 06}{14655}$

\section{CALCULATION SHEET}

\begin{tabular}{|c|c|c|c|c|c|c|c|c|c|c|c|c|c|c|c|c|c|c|c|c|c|c|c|c|c|c|}
\hline \multirow{2}{*}{$\begin{array}{c}\text { Sampling } \\
\text { Area } \\
\end{array}$} & \multirow{2}{*}{$\begin{array}{l}\text { Sample } \\
\text { Number }\end{array}$} & \multirow{2}{*}{$\begin{array}{c}\text { Sample } \\
\text { Date } \\
\end{array}$} & \multicolumn{3}{|c|}{ Americium-241 } & \multicolumn{3}{|c|}{ Cesium-137 } & \multicolumn{3}{|c|}{ Cobalt-60 } & \multicolumn{3}{|c|}{ Europium-152 } & \multicolumn{3}{|c|}{ Europium-154 } & \multicolumn{3}{|c|}{ Europium-155 } & \multicolumn{3}{|c|}{ Nickel-63 } & \multicolumn{3}{|c|}{ Tritium } \\
\hline & & & & Q & MD & & & $\bar{M}$ & - & 5 & 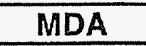 & & Q & & & Q & (10 & & Q & $\overline{M D}$ & & & & $\mathrm{Ci} / \mathrm{g}$ & 01 & MDA \\
\hline $\mathrm{A} 1$ & & & & U & 0 & $2.24 \mathrm{E}+00$ & & & & $U$ & & & $U$ & & & $U$ & & & 11 & & & $\bar{U}$ & & & $\bar{U}$ & \\
\hline$\hat{1}$ & & & & $U$ & & & $\bar{U}$ & & & 11 & & & $U$ & & & 14 & & & 10 & & & & & & U & \\
\hline & & & & & & & 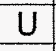 & & & U & & & & & & 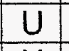 & & & $\bar{U}$ & & & & & & U & \\
\hline $\mathrm{A} 2$ & $J 13 \mathrm{D} J 7$ & $9 / 7 / 06$ & $5.6 \mathrm{E}-02$ & $u$ & $7.6 \mathrm{E}-02$ & -02 & & $1.7 \mathrm{E}-02$ & $4.6 \mathrm{E}-02$ & $U$ & $4.6 \mathrm{E}-02$ & $3.4 \mathrm{E}-02$ & U & 3.4 & $5.3 E-02$ & U & 2 & $=-02$ & $U$ & $3.8 \mathrm{E}-02$ & $9.46 \mathrm{E}-01 \quad \mid$ & U & 0 & 01 & $U$ & 2.3 \\
\hline olica & 13DJ8 & $9 / 7 / 06$ & $9.9 \mathrm{E}-02$ & $u$ & $1.1 \mathrm{E}-0$ & $4.0 \mathrm{E}-02$ & & $1.9 \mathrm{E}-02$ & $1.7 \mathrm{E}-02$ & U & $1.7 \mathrm{E}-0$ & 4.3E-02 & U & $1.3 \mathrm{E}-0$ & $5.1 E-02$ & U & $5.1 \mathrm{E}-02$ & $6.1 \mathrm{E}-02$ & U & $1 \mathrm{E}-02$ & $.01 E+00$ & U & $3.9 \mathrm{E}+00$ & $1.77 \mathrm{E}-01$ & $u$ & .00 \\
\hline
\end{tabular}
8 J13DJ7 $9 / 7 / 06$
Calc No. $0600 \mathrm{X}-\mathrm{CA}-\mathrm{V} 0060$ Checked J.M. Capron gme
Rev. No. $\frac{0}{11 / 01 / 06}$

11 Statistical Computation Input Data

\begin{tabular}{|c|c|c|c|c|c|c|c|c|c|c|}
\hline \multirow[t]{5}{*}{$\begin{array}{c}\begin{array}{c}\text { Sampling } \\
\text { Area }\end{array} \\
\end{array}$} & \multirow{5}{*}{\begin{tabular}{c|}
$\begin{array}{c}\text { Sample } \\
\text { Number }\end{array}$ \\
J13DJ6 \\
J13DJ9 \\
J13DK0 \\
13DJ7/J13DJ8 \\
\end{tabular}} & \multirow{5}{*}{$\begin{array}{c}\begin{array}{c}\text { Sample } \\
\text { Date }\end{array} \\
9 / 7 / 106 \\
9 / 1 / 16 \\
9 / 7 / 16 \\
9 / 706 \\
\end{array}$} & \multirow{2}{*}{$\begin{array}{l}\text { Americium-241 } \\
\text { pCil/g } \\
-1.0 \mathrm{E}-01\end{array}$} & \multirow{2}{*}{\begin{tabular}{|c|}
$\begin{array}{c}\text { Cesium-137 } \\
\text { pCi/g }\end{array}$ \\
$2.24 \mathrm{E}+00$ \\
\end{tabular}} & \multirow{2}{*}{\begin{tabular}{|c|}
$\begin{array}{c}\text { Cobalt-60 } \\
\text { pCi/g }\end{array}$ \\
$1.4 \mathrm{E}-02$ \\
\end{tabular}} & \multirow{2}{*}{\begin{tabular}{|l} 
Europium-152 \\
pCi/g
\end{tabular}} & \multirow{2}{*}{\begin{tabular}{|}
$\begin{array}{c}\text { Europium-154 } \\
\mathrm{pCilg} / \mathrm{g}\end{array}$ \\
\end{tabular}} & $\begin{array}{c}\text { Europium-155 } \\
\text { pCi/g }\end{array}$ & \multirow[t]{2}{*}{$\begin{array}{c}\begin{array}{c}\text { Nickel-63 } \\
\text { pCil/g }\end{array} \\
\end{array}$} & \multirow{2}{*}{\begin{tabular}{|c|}
$\mid \begin{array}{c}\text { Tritium } \\
\text { pCi/g }\end{array}$ \\
$-4.29 \mathrm{E}-01$ \\
\end{tabular}} \\
\hline & & & & & & & & $3.4 \mathrm{E}-02$ & & \\
\hline & & & $-3.7 \mathrm{E}-02$ & $2.4 \mathrm{E}-02$ & $2.4 \mathrm{E}-02$ & $6.5 \mathrm{E}-02$ & $8.5 \mathrm{E}-02$ & $9.0 \mathrm{E}-02$ & $8.08 \mathrm{E}-01$ & $-8.46 E-0$ \\
\hline & & & $8.1 \mathrm{E}-01$ & $1.1 \mathrm{E}-02$ & $1.2 \mathrm{E}-02$ & $2.4 \mathrm{E}-02$ & $3.9 \mathrm{E}-02$ & $2.5 \mathrm{E}-02$ & $-5.40 \mathrm{E}-01$ & $-2.26 \mathrm{E}-01$ \\
\hline & & & $7.8 \mathrm{E}-02$ & $3.7 \mathrm{E}-02$ & $1.6 \mathrm{E}-02$ & $1.9 \mathrm{E}-02$ & $2.6 \mathrm{E}-02$ & $2.5 \mathrm{E}-02$ & $-3.20 \mathrm{E}-02$ & $3.04 \mathrm{E}-01$ \\
\hline
\end{tabular}$$
18
$$

20 Statistical Computations

\begin{tabular}{|c|c|c|c|c|c|c|c|c|}
\hline & & \multicolumn{2}{|c|}{ Americium-241 } & \multicolumn{2}{|l|}{ Cesium-137 } & \multicolumn{3}{|l|}{ Cobalt-60 } \\
\hline & $95 \%$ UCL based on & $\begin{array}{rr}\text { Radionu } \\
\text { Use non } \\
\text { st }\end{array}$ & $\begin{array}{l}\text { clide data set. } \\
\text { parametric z- } \\
\text { atistic. }\end{array}$ & \begin{tabular}{|c|} 
Radionuclid \\
nonparame
\end{tabular} & $\begin{array}{l}\text { de data set. Use } \\
\text { etric } z \text {-statistic. }\end{array}$ & $\begin{array}{r}\text { Radionuc } \\
\text { Use nonf } \\
\text { st }\end{array}$ & $\begin{array}{l}\text { clide } \\
\text { ipara } \\
\text { tatist }\end{array}$ & $\begin{array}{l}\text { data set. } \\
\text { ametric } z- \\
\text { tic. }\end{array}$ \\
\hline & & 4 & & 4 & & 4 & & \\
\hline & $\%<$ Detection limit & $75 \%$ & & $50 \%$ & & $100 \%$ & & \\
\hline & mean & $\begin{array}{ll}\text { n } 1.9 \mathrm{E}-01 \\
\end{array}$ & & $5.8 \mathrm{E}-01$ & & $1.6 \mathrm{E}-02$ & & \\
\hline & st. dev. & $4.2 \mathrm{E}-01$ & & $1.1 E+00$ & & $5.5 \mathrm{E}-03$ & & \\
\hline & Z-statistic & 1.645 & & 1.645 & & 1.645 & & \\
\hline & $95 \%$ UCL on mean & \begin{tabular}{l|l}
$n$ & $5.4 \mathrm{E}-01$ \\
\end{tabular} & & $1.5 \mathrm{E}+00$ & & $2.1 \mathrm{E}-02$ & & \\
\hline & max value & e $8.1 \mathrm{E}-01$ & & $2.2 E+00$ & & $4.8 \mathrm{E}-02$ & U & \\
\hline & Statistical value & $\begin{array}{l}\mathrm{e} \quad 5.4 \mathrm{E}-01 \\
\end{array}$ & & $1.5 \mathrm{E}+00$ & & 2.1E-02 & $\mathrm{U}$ & \\
\hline & Background & $\mathrm{NA}$ & & NA & & $\mathrm{NA}$ & & \\
\hline & ch ho & $5.4 \mathrm{E}-\mathrm{C}$ & & $1.5 \mathrm{E}+0$ & & & & \\
\hline
\end{tabular}

\begin{tabular}{|c|c|c|c|c|c|}
\hline \multicolumn{3}{|c|}{ Europium-152 } & \\
\hline $\begin{array}{l}\text { Radionuclic } \\
\text { nonparam }\end{array}$ & etric & $\begin{array}{l}\text { lata set. Use } \\
\text { c z-statistic. }\end{array}$ & $\begin{array}{l}\text { Radiont } \\
\text { Use no } \\
\text { S }\end{array}$ & $\begin{array}{l}\text { lide } \\
\text { liare } \\
\text { atist }\end{array}$ & $\begin{array}{l}\text { data set. } \\
\text { ametric z- } \\
\text { tic. }\end{array}$ \\
\hline 4 & & & 4 & & \\
\hline$\frac{4}{100 \%}$ & & & $100 \%$ & & \\
\hline $3.7 \mathrm{E}-02$ & & & $\frac{1007}{5.0 \mathrm{E}-02}$ & & \\
\hline $2.1 \mathrm{E}-02$ & & & $2.5 \mathrm{E}-02$ & & \\
\hline 1.645 & & & 1.645 & & \\
\hline $5.5 \mathrm{E}-02$ & & & $7.0 \mathrm{E}-02$ & & \\
\hline $1.3 \mathrm{E}-01$ & u & & $1.7 \mathrm{E}-01$ & $u$ & \\
\hline $5.5 \mathrm{E}-02$ & u & & $7.0 \mathrm{E}-02$ & $\frac{\widetilde{U}}{\mathrm{U}}$ & \\
\hline NA & & & NA & & \\
\hline $5.5 \mathrm{E}-02$ & & & $7.0 \mathrm{E}-02$ & & \\
\hline
\end{tabular}

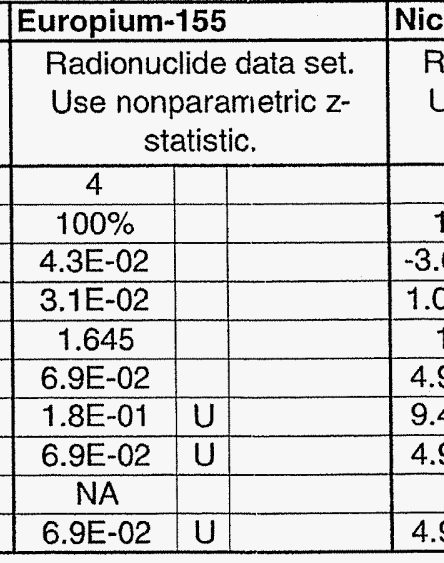

Nickel-63

Tritium Radionuclide data set. Radionuclide data set. $\mathrm{NA}$ \begin{tabular}{|c|c|}
\hline NA \\
\hline $2.1 E-02$ \\
\hline
\end{tabular} 


\section{Washington Closure Hanford}

Originator K. A. Anselm lLai

Project 300 Area Field Remediation

Subject Cleanup Verification 95\% UCL Calculations for 618-2 Shallow Zone/Deep Zone/Overburden and 618-2, 618-3, 618-8 Staging Pile and Decon Pad Footpin
CALCULATION SHEET

Date $11 / 01 / 06$

Job No.

Calc. No $0600 \mathrm{X}-\mathrm{CA}$-V0060

Rev. No. $\frac{0}{110100}$

$\frac{11 / 01 / 06}{50 f 21}$

1618-2 Shallow Zone Sample Data

\begin{tabular}{|c|c|c|c|c|c|c|c|c|c|c|c|c|c|c|c|c|c|c|c|c|c|c|c|}
\hline \multirow{2}{*}{$\begin{array}{c}\text { Sampling } \\
\text { Area }\end{array}$} & \multirow{2}{*}{$\begin{array}{l}\text { Sample } \\
\text { Number }\end{array}$} & \multirow{2}{*}{$\begin{array}{c}\text { Sample } \\
\text { Date }\end{array}$} & \multicolumn{3}{|c|}{ Plutonium-238 } & \multicolumn{3}{|c|}{ Plutonium-239/240 } & \multicolumn{3}{|c|}{ Plutonium-241 } & \multicolumn{3}{|c|}{ Strontium-90 } & \multicolumn{3}{|c|}{ Uranium-233/234 } & \multicolumn{3}{|c|}{ Uranium-235 } & \multicolumn{3}{|c|}{ Uranium-238 } \\
\hline & & & $\mathrm{pCi} / \mathrm{g}$ & $\begin{array}{lll}0 & & \\
\end{array}$ & MDA & $\mathrm{pCi} / \mathrm{g}$ & Q & MDA & $\mathrm{pCi} / \mathrm{g}$ & a & MDA & $\mathrm{pCi} / \mathrm{g}$ & Q & MDA & $\mathrm{OCi} / \mathrm{g}$ & a & MDA & $\mathrm{pCi} / \mathrm{g}$ & Q & MDA & $\mathrm{pCi} / \mathrm{g}$ & Q & $\overline{M D A}$ \\
\hline At & J13DJ6 & $9 / 7 / 06$ & $5.1 \mathrm{E}-$ & $\bar{U}$ & $4.9 \mathrm{E}-01$ & $x$ & U & $3.9 \mathrm{E}-01$ & & $\pi$ & & pong & $\bar{U}$ & & (1) & & & & 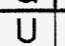 & & $.90 \mathrm{E}+00$ & & $1.7 \mathrm{E}-01$ \\
\hline$A^{\prime}$ & & & $=$ & $u$ & & $6.9 \mathrm{E}-$ & $\mathrm{U}$ & & 01 & U & & & $u$ & & & & & & $U$ & & & & \\
\hline A & & & 0 & $u$ & 4. & & & & 0 & $\mathrm{U}$ & 2.4 & & $U$ & & & & & & $\mathrm{u}$ & & & & 01 \\
\hline A2 & J1. & प्र/r & $5.9 \mathrm{E}-02$ & $U$ & $4.5 E-01$ & $8.24 \mathrm{E}-01$ & & $4.5 \mathrm{E}-01$ & $1.42 \mathrm{E}+01$ & $\mathrm{U}$ & $2.0 \mathrm{E}+01$ & $-1.4 \mathrm{E}-02$ & $U$ & $2.2 \mathrm{E}-01$ & $1.71 \mathrm{E}+00$ & & $1.7 \mathrm{E}-01$ & $2.14 \mathrm{E}-01$ & & $2.1 \mathrm{E}-01$ & $1.77 \mathrm{E}+00$ & & $1.7 \mathrm{E}-01$ \\
\hline Duplicate of & J13DJ8 & $9 / 7 / 06$ & $6.8 \mathrm{E}-02$ & $u$ & $2.6 \mathrm{E}-01$ & $7.18 \mathrm{E}-01$ & & $2.6 \mathrm{E}-01$ & $9.65 \mathrm{E}+00$ & $u$ & $1.8 \mathrm{E}+01$ & $-2.4 \mathrm{E}-02$ & $\mathrm{U}$ & $2.5 \mathrm{E}-01$ & $1.83 \mathrm{E}+00$ & & 2.3E-01 & $1.11 \mathrm{E}-01$ & $\mathrm{u}$ & $2.8 \mathrm{E}-01$ & $1.89 \mathrm{E}+00$ & & 2.3E-01 \\
\hline
\end{tabular}

11 Statistical Computation Input Dat

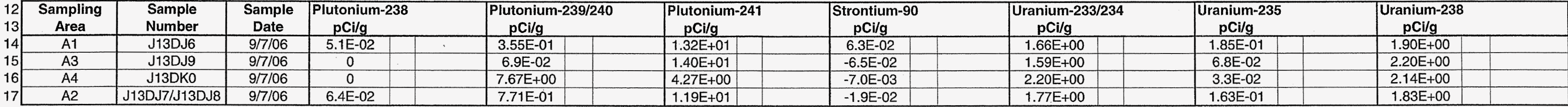

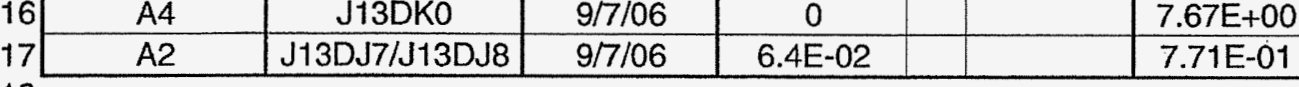
18

20 Statistical Computations

\begin{tabular}{|c|c|c|c|c|c|c|c|c|c|}
\hline & ( & Plutonium- & & & Plutonium & $239 / 240$ & Plutonium & $1-241$ & \\
\hline 22 & $95 \%$ UCL based on & $\begin{array}{c}\text { Radionuclic } \\
\text { nonparam }\end{array}$ & $\begin{array}{l}\text { edd } \\
\text { etric }\end{array}$ & $\begin{array}{l}\text { data set. Use } \\
\text { c } \text {-statistic. }\end{array}$ & $\begin{array}{c}\text { Radionucli } \\
\text { nonparam }\end{array}$ & $\begin{array}{l}\text { e data set. Use } \\
\text { etric z-statistic. }\end{array}$ & $\begin{array}{l}\text { Radionuo } \\
\text { Use non } \\
\text { st }\end{array}$ & $\begin{array}{l}\text { clide } \\
\text { para } \\
\text { tatist }\end{array}$ & $\begin{array}{l}\text { data set. } \\
\text { ametric } z- \\
\text { tic. }\end{array}$ \\
\hline 23 & $\bar{N}$ & 4 & & & 4 & & 4 & & \\
\hline 24 & $\%<$ Detection limit & $100 \%$ & & & $50 \%$ & & $100 \%$ & & \\
\hline 25 & mean & $2.9 \mathrm{E}-02$ & & & $2.2 E+00$ & & $1.08 \mathrm{E}+01$ & & \\
\hline 26 & st. dev. & $3.3 \mathrm{E}-02$ & & & $3.6 \mathrm{E}+00$ & & $4.47 \mathrm{E}+00$ & & \\
\hline 27 & Z-statistic & 1.645 & & & 1.645 & & 1645 & & \\
\hline 28 & $95 \%$ UCL on mean & $5.6 \mathrm{E}-02$ & & & $5.2 \mathrm{E}+00$ & & $1.45 \mathrm{E}+01$ & & \\
\hline 29 & max value & $6.8 \mathrm{E}-02$ & $u$ & & $7.7 \mathrm{E}+00$ & & $1.42 E+01$ & $\bar{u}$ & \\
\hline 30 & Statistical value & $5.6 \mathrm{E}-02$ & u & & $5.2 \mathrm{E}+00$ & & $1.45 E+01$ & $\mathrm{u}$ & \\
\hline 31 & Backaround & $N A$ & & & $N A$ & & $N A$ & & \\
\hline $32>>>>>>3$ & Statistical value above backaround & $5.6 \mathrm{E}-02$ & $u$ & & $5.2 \mathrm{~F}+00$ & & $145 \mathrm{~F}+01$ & $u$ & \\
\hline
\end{tabular}

Uranium-233/234

Uranium-235

Uranium-238 \begin{tabular}{c|c|c|c}
$\begin{array}{c}\text { Radionuclide data set. Use } \\
\text { nonparametric z-statistic. }\end{array}$ & $\begin{array}{c}\text { Radionuclide data set. Use } \\
\text { nonparametric z-statistic. }\end{array}$ & $\begin{array}{c}\text { Radionuclide data set. Use } \\
\text { nonparametric z-statistic. }\end{array}$ & $\begin{array}{c}\text { Radionuclide data set. Use } \\
\text { nonparametric z-statistic. }\end{array}$ \\
\hline
\end{tabular}

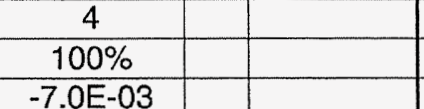



and 618-2, 618-3, 618-8 Staging Pile and Decon Pad Footprint

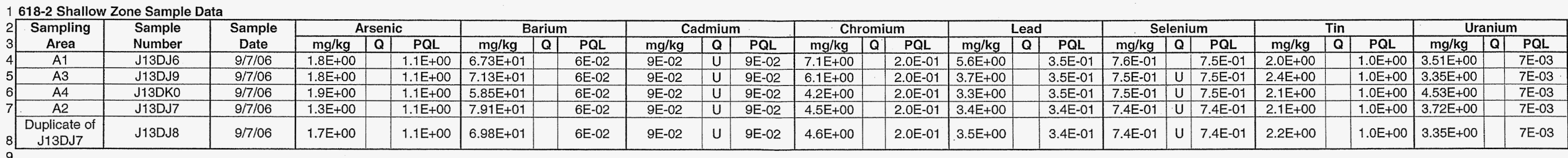

11 Statistical Computation Input Data

\begin{tabular}{|c|c|c|c|c|c|c|c|c|c|c|}
\hline \begin{tabular}{l|l}
12 & Sampling \\
13 & Area
\end{tabular} & \multirow{2}{*}{$\begin{array}{l}\begin{array}{l}\text { Sample } \\
\text { Number }\end{array} \\
\text { J13DJ6 }\end{array}$} & \multirow{2}{*}{$\begin{array}{c}\text { Sample } \\
\text { Date } \\
9 / 7 / 06 \\
\end{array}$} & $\begin{array}{c}\text { Arsenic } \\
\mathrm{mg} / \mathrm{kg}\end{array}$ & $\begin{array}{c}\text { Barium } \\
\mathrm{mg} / \mathrm{kg}\end{array}$ & Cadmium & Chromium & $\begin{array}{l}\text { Lead } \\
\text { ma/k }\end{array}$ & \begin{tabular}{|} 
Selenium \\
$\mathrm{mg} / \mathrm{kq}$
\end{tabular} & \multirow[t]{2}{*}{$\begin{array}{c}\text { Tin } \\
\mathrm{mg} / \mathrm{kg}\end{array}$} & $\begin{array}{l}\text { Uranium } \\
\mathrm{mg} / \mathrm{kg}\end{array}$ \\
\hline$1 4 \longdiv { A 1 }$ & & & \begin{tabular}{|l|l|l}
$\mathrm{mgkg}$ \\
$1.8 \mathrm{E}+00$ \\
\end{tabular} & $\frac{\text { migkg }}{6.73 \mathrm{E}+01}$ & \begin{tabular}{|l|}
$\mathrm{mg} / \mathrm{kg}$ \\
$5 \mathrm{E}-02$ \\
\end{tabular} & \begin{tabular}{|l|}
$\mathrm{mg} / \mathrm{kg}$ \\
$7.1 \mathrm{k}+00$ \\
\end{tabular} & \begin{tabular}{|l|l} 
mgg/kg \\
$5.6 \mathrm{E}+00$
\end{tabular} & \begin{tabular}{|l|} 
migkg \\
$7.6 \mathrm{E}-01$ \\
\end{tabular} & & \begin{tabular}{|l|l}
$3.51 E+00$ \\
\end{tabular} \\
\hline A3 & J13DJ9 & $9 / 7 / 106$ & $1.8 \mathrm{E}+00$ & $7.13 E+01$ & $5 \mathrm{E}-02$ & $6.1 \mathrm{E}+00$ & $3.7 E+00$ & \begin{tabular}{|l|}
$3.8 \mathrm{E}-01$ \\
\end{tabular} & $2.4 \mathrm{E}+00$ & $3.35 E+00$ \\
\hline A4 & J13DKO & $9 / 7 / 06$ & $1.9 \mathrm{E}+00$ & $5.85 E+01$ & $5 \mathrm{E}-02$ & 年.1E+0 & \begin{tabular}{|l|}
$0.1+00$ \\
$3.3 E+00$
\end{tabular} & \begin{tabular}{|l|}
$3.0 \mathrm{E}-01$ \\
$3 \mathrm{E}-01$ \\
\end{tabular} & $2.1 \mathrm{E}+00$ & \begin{tabular}{|l}
$4.53 E+00$ \\
\end{tabular} \\
\hline $\begin{array}{ll}A_{4} \\
A^{2}\end{array}$ & \begin{tabular}{|l|l}
$J 13 D J 7 / J 13 D J 8$ \\
\end{tabular} & $9 / 7 / 06$ & $1.5 \mathrm{E}+00$ & $\begin{array}{l}\frac{0.05 \mathrm{C}+\mathrm{U}}{7.45 \mathrm{E}+01} \\
\end{array}$ & $\begin{array}{l}3 \mathrm{se-06} \\
5 \mathrm{E}-02 \\
\end{array}$ & 年. $4.6 \mathrm{E}+00$ & \begin{tabular}{|l|l|}
$0.52+00$ \\
$3.5 \mathrm{E}+00$ \\
\end{tabular} & \begin{tabular}{|l|}
$3.7 \mathrm{E}-01$ \\
\end{tabular} & $2.2 \mathrm{E}+00$ & $3.54 \mathrm{E}+00$ \\
\hline
\end{tabular}

20 Statistical Computations

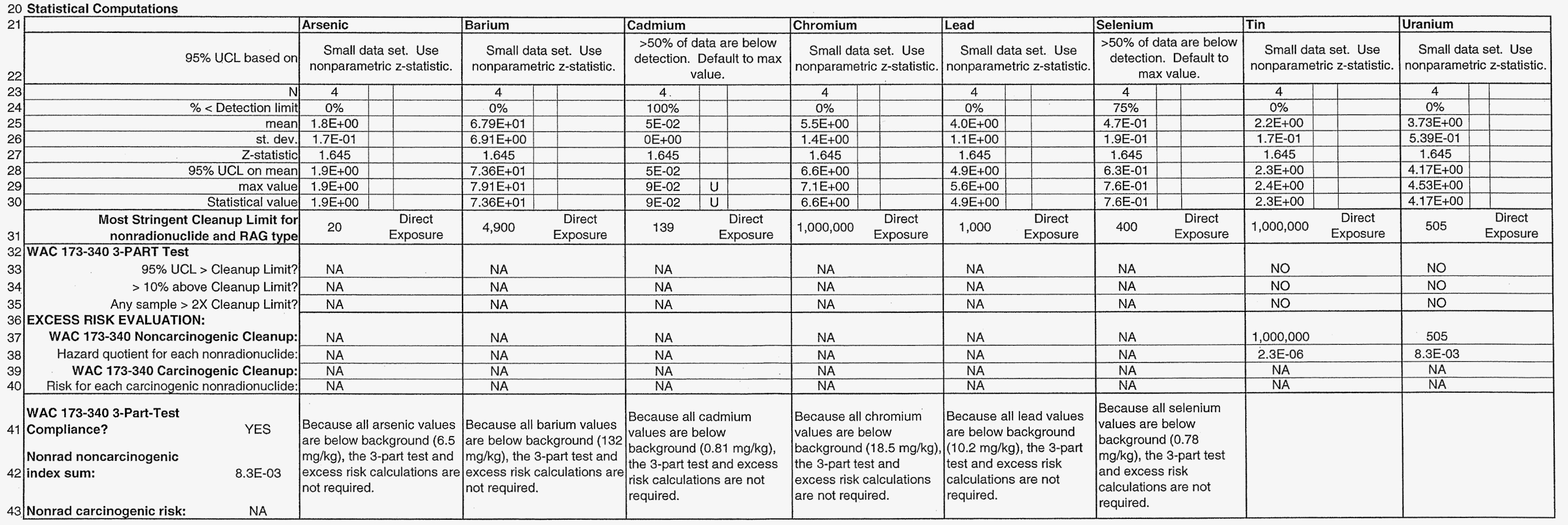




\section{Washington Closure Hanford}

Originator K. A. Anselm icaa

Project

Calculations for 618-2 Shallow Zone/Deep Zone/Overburden, and 618-2, 618-3, 618-8 Staging Pile and Decon Pad Footprint
CALCULATION SHEET

Date $11 / 01 / 06$

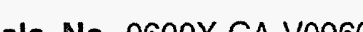

Checked J.M. Capron QmC
Rev. No. $\quad 0$

Date $\frac{0}{71 / 01 / 06}$

\begin{tabular}{|c|c|c|c|c|c|c|c|c|c|c|c|c|c|c|c|c|c|c|c|c|c|c|c|c|c|}
\hline \multirow{2}{*}{$\begin{array}{ccc}2 & \text { Sampling } \\
3 & \text { Area }\end{array}$} & \multirow{2}{*}{$\begin{array}{l}\text { Sample } \\
\text { Number }\end{array}$} & \multirow{2}{*}{$\begin{array}{c}\text { Sample } \\
\text { Date }\end{array}$} & \multicolumn{2}{|c|}{ Americium-241 } & \multicolumn{3}{|c|}{ Cesium-137 } & \multicolumn{3}{|c|}{ Cobalt-60 } & \multicolumn{3}{|c|}{ Europium-152 } & \multicolumn{3}{|c|}{ Europium-154 } & \multicolumn{3}{|c|}{ Europium-155 } & \multicolumn{3}{|c|}{ Nickel-63 } & \multicolumn{3}{|c|}{ Tritium } \\
\hline & & & & MDA & $\mathrm{Ci} / \mathrm{g}$ & 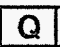 & MDA & $\mathrm{Ci} / \mathrm{g}$ & Q & MDA & $\mathrm{pCi} / \mathrm{g}$ & a & MDA & $\mathrm{pCi} / \mathrm{g}$ & Q & MDA & $\mathrm{pCi} / \mathrm{g}$ & Q & MDA & $\mathrm{pCi} / \mathrm{g}$ & Q & MDA & $\mathrm{pCi} / \mathrm{g}$ & Q & MDA \\
\hline$A 1$ & J13DJ4 & $9 / 7 / 06$ & $3.08 \mathrm{E}-01$ & & $86 \mathrm{E}-01$ & & & $1.7 \mathrm{E}-02$ & $U$ & 1.7 & & U & & & $U$ & & & $U$ & & & 11 & & $7.9 \mathrm{E}-02$ & $\mathrm{U}$ & $2.5 \mathrm{E}+00$ \\
\hline$A 3$ & & & & & $5.90 \mathrm{E}-02$ & & & $3.7 \mathrm{E}-02$ & $U$ & $3.7 \mathrm{E}$ & 2 & $U$ & & $1.2 \mathrm{E}$ & $u$ & & & 11 & & & & & & & +00 \\
\hline $\mathrm{A}$ & & & & & & & & & $u$ & & & U & & $1.8 \mathrm{E}-01$ & $u$ & & & $u$ & & 4.06 & $\bar{U}$ & & $3.59 \mathrm{E}-01$ & $\bar{U}$ & \\
\hline$\hat{A}$ & JISLAT & 9//106 & $7.94 E+00$ & $6.1 \mathrm{t}-02$ & $2.09 \mathrm{E}-01$ & & 4.6 & $3.7 E-02$ & $u$ & $3.1 \mathrm{E}-02$ & $9.9 \mathrm{E}-02$ & $U_{1}$ & $0 \leq$ & 01 & $u$ & $1.2 \mathrm{E}-01$ & 02 & 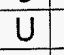 & 9.6 & 4.97E-01 & U & $3.6 \mathrm{E}+00$ & $4.54 \mathrm{E}-01$ & U & $2.5 \mathrm{E}+00$ \\
\hline 1130 in & J13DJ1 & $9 / 7 / 06$ & $82 \mathrm{E}+00$ & 4.7E-02 & $3.92 E-01$ & & $4.6 \mathrm{E}-02$ & $3.2 E-02$ & & $3.2 E-02$ & $9.2 \mathrm{E}-02$ & & $2 \mathrm{E}-02$ & IE- $C$ & & 1E-01 & $9 \mathrm{E}-0$ & & 9E-02 & $68 \mathrm{E}-01$ & U & $6 \mathrm{E}+00$ & $5.80 \mathrm{E}-01$ & U & $2.4 \mathrm{E}+00$ \\
\hline
\end{tabular}

8 J13DJ0

J13DJ1

\begin{tabular}{ll|l}
$9 / 7 / 06$ & $6.82 \mathrm{E}+00$
\end{tabular}

1 Statistical Computation Input Data

\begin{tabular}{|c|c|c|c|c|c|c|c|c|c|c|}
\hline \multirow[t]{2}{*}{\begin{tabular}{|c|}
$\begin{array}{c}\text { Sampling } \\
\text { Area }\end{array}$ \\
\end{tabular}} & $\begin{array}{l}\text { Sample } \\
\text { Number }\end{array}$ & $\begin{array}{c}\text { Sample } \\
\text { Date }\end{array}$ & $\begin{array}{l}\text { Americium-241 } \\
\mathrm{pCi} / \mathrm{g}\end{array}$ & $\begin{array}{c}\text { Cesium-137 } \\
\mathrm{pCli} / \mathrm{g}\end{array}$ & $\begin{array}{c}\text { Cobalt-60 } \\
\text { pCi/g }\end{array}$ & $\begin{array}{c}\text { Europium } \\
\mathrm{pCi} / \mathrm{g}\end{array}$ & $\begin{array}{c}\text { Europium } \\
\mathrm{pCi} / \mathrm{g}\end{array}$ & $\begin{array}{c}\text { Europium } \\
\mathrm{pCi} / \mathrm{g}\end{array}$ & $\begin{array}{c}\text { Nickel-63 } \\
\text { pCi/g }\end{array}$ & \begin{tabular}{|c|}
$\begin{array}{r}\text { Tritium } \\
\text { pCi/g }\end{array}$ \\
\end{tabular} \\
\hline & J13DJ4 & $9 / 7 / 06$ & $3.08 \mathrm{E}-01$ & $7.86 \mathrm{E}-01$ & \begin{tabular}{|l|}
$8.5 \mathrm{E}-03$ \\
\end{tabular} & 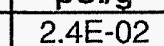 & $2.6 \mathrm{E}-02$ & $2.0 \mathrm{E}-02$ & $1.72 \mathrm{E}-01$ & $7.90 \mathrm{E}-02$ \\
\hline A3 & J13DJ2 & $9 / 7 / 06$ & \begin{tabular}{|l|}
$2.77 \mathrm{E}+00$ \\
\end{tabular} & \begin{tabular}{|l|}
$5.90 \mathrm{E}-02$ \\
\end{tabular} & $\begin{array}{l}1.9 E-02 \\
\end{array}$ & $4.5 \mathrm{E}-02$ & $6.0 \mathrm{E}-02$ & $4.3 \mathrm{E}-02$ & $|-1.48 \mathrm{E}+00|$ & $6.24 \mathrm{E}-01$ \\
\hline A4 & J13DJ3 & $9 / 7 / 06$ & $5.45 \mathrm{E}+00$ & $1.04 E+00$ & $2.5 \mathrm{E}-02$ & $80 \mathrm{E}-02$ & $9.0 \mathrm{E}-02$ & $1.8 \mathrm{E}-01$ & $-4.06 E-01$ & $8.59 \mathrm{E}-01$ \\
\hline$\frac{\mathrm{A}_{4}}{\mathrm{~A}^{2}}$ & 3DJO/J13D & $9 / 7 / 06$ & \begin{tabular}{|l|}
$.430 \mathrm{~L}+00$ \\
$738 \mathrm{E}+00$
\end{tabular} & \begin{tabular}{|l|}
$1.04 L+0$ \\
$301 E-01$
\end{tabular} & $17 \mathrm{E}-02$ & $\begin{array}{l}.0 E-02 \\
4.8 E-02\end{array}$ & $\frac{9.0 \mathrm{~L}-\mathrm{UL}}{5.8 \mathrm{E}-02}$ & $\begin{array}{l}1.0 \mathrm{~L}-\mathrm{U} \\
4.6 \mathrm{E}-02\end{array}$ & $|1.86 \mathrm{E}-01|$ & \begin{tabular}{|l|l|}
$5.17 \mathrm{E}-01$ \\
\end{tabular} \\
\hline
\end{tabular}

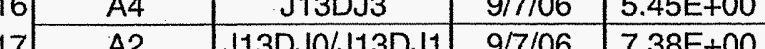

20 Statistical Computations

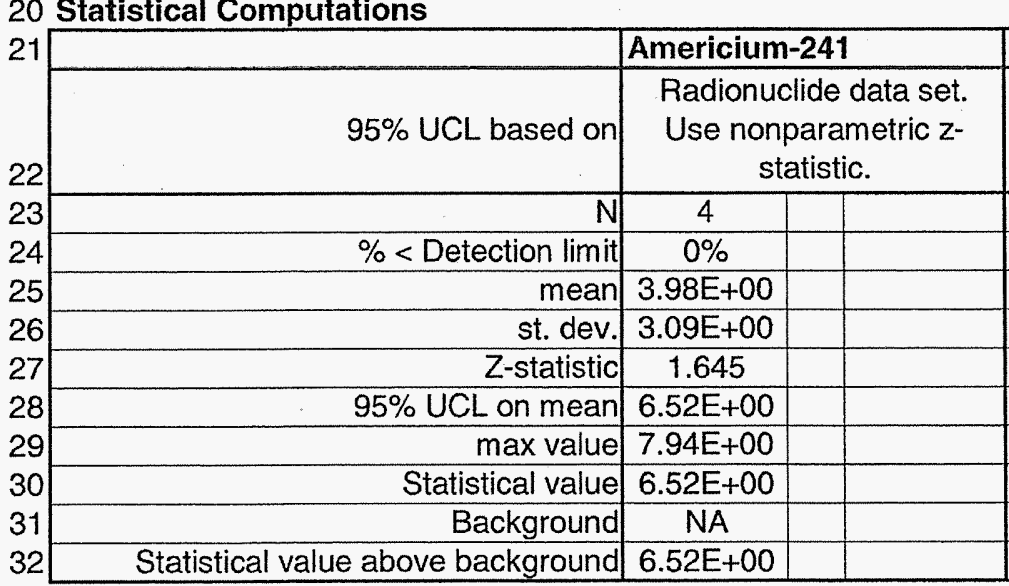

\begin{tabular}{|c|c|c|c|c|}
\hline \multirow{2}{*}{\multicolumn{2}{|c|}{\begin{tabular}{|l|} 
Cesium-137 \\
Radionuclide data set. Use \\
nonparametric z-statistic. \\
\end{tabular}}} & \multirow{2}{*}{\multicolumn{3}{|c|}{$\begin{array}{l}\text { Cobalt-60 } \\
\text { Radionuclide data set } \\
\text { Use nonparametric z- } \\
\text { statistic. } \\
\end{array}$}} \\
\hline & & & & \\
\hline 4 & & & & \\
\hline $0 \%$ & & $100 \%$ & & \\
\hline $5.46 \mathrm{E}-01$ & & $1.7 \mathrm{E}-02$ & & \\
\hline $4.47 \mathrm{E}-01$ & & $6.6 \mathrm{E}-03$ & & \\
\hline 1.645 & & 1.645 & & \\
\hline $9.14 \mathrm{E}-01$ & & $2.3 \mathrm{E}-02$ & & \\
\hline $1.04 \mathrm{E}+00$ & & $4.9 \mathrm{E}-02$ & $\mathrm{U}$ & \\
\hline $9.14 \mathrm{E}-01$ & & $2.3 \mathrm{E}-02$ & U & \\
\hline NA & & NA & & \\
\hline & & & & \\
\hline
\end{tabular}

\begin{tabular}{|c|c|c|c|c|c|c|c|c|}
\hline \multirow{2}{*}{\multicolumn{3}{|c|}{\begin{tabular}{|l|} 
Europium-152 \\
Radionuclide data set. Use \\
nonparametric z-statistic.
\end{tabular}}} & \multirow{2}{*}{\multicolumn{3}{|c|}{$\begin{array}{l}\text { Europium-154 } \\
\text { Radionuclide data set. } \\
\text { Use nonparametric z- } \\
\text { statistic. }\end{array}$}} & \multirow{2}{*}{\multicolumn{3}{|c|}{$\begin{array}{l}\text { Europium-155 } \\
\text { Radionuclide data set. } \\
\text { Use nonparametric z- } \\
\text { statistic. }\end{array}$}} \\
\hline & & & & & & & & \\
\hline 4 & & & 4 & & & & & \\
\hline $100 \%$ & & & 1 & & & $100 \%$ & & \\
\hline $4.9 \mathrm{E}-02$ & & & $5.8 \mathrm{E}-02$ & & & \begin{tabular}{|l|l}
$7.1 \mathrm{E}-02$ \\
\end{tabular} & & \\
\hline $2.3 \mathrm{E}-02$ & & & $2.6 \mathrm{E}-02$ & & & $\begin{array}{l}7.0 \mathrm{E}-02 \\
\end{array}$ & & \\
\hline 1.645 & & & 1.645 & & & 1.645 & & \\
\hline $6.8 \mathrm{E}-02$ & & & $8.0 \mathrm{E}-02$ & & & $1.3 \mathrm{E}-01$ & & \\
\hline $1.6 \mathrm{E}-01$ & U & & $1.8 \mathrm{E}-01$ & U & & $3.5 \mathrm{E}-01$ & $\mathrm{U}$ & \\
\hline $6.8 \mathrm{E}-02$ & U & & $8.0 \mathrm{E}-02$ & U & & $1.3 \mathrm{E}-01$ & U & \\
\hline NA & & & NA & & & NA & & \\
\hline $6.8 \mathrm{~F}-02$ & u & & $8.0 \mathrm{E}-02$ & $\mathrm{u}$ & & \begin{tabular}{|c|}
$1.3 \mathrm{E}-01$ \\
\end{tabular} & $\mathrm{u}$ & \\
\hline
\end{tabular}

\begin{tabular}{|c|c|c|c|c|}
\hline \multirow{2}{*}{\multicolumn{2}{|c|}{\begin{tabular}{|l|} 
Nickel-63 \\
Radionuclide data set. \\
Use nonparametric z- \\
statistic. \\
\end{tabular}}} & \multirow{2}{*}{\multicolumn{3}{|c|}{$\begin{array}{l}\text { Tritium } \\
\text { Radionuclide data set. } \\
\text { Use nonparametric z- } \\
\text { statistic. }\end{array}$}} \\
\hline & & & & \\
\hline \begin{tabular}{l|l}
4 \\
\end{tabular} & & & & \\
\hline $100 \%$ & & $100 \%$ & & \\
\hline$-3.82 \mathrm{E}-01$ & & $5.20 \mathrm{E}-01$ & & \\
\hline $7.82 \mathrm{E}-01$ & & $3.27 \mathrm{E}-01$ & & \\
\hline 1.645 & & 1.645 & & \\
\hline $2.61 \mathrm{E}-01$ & & $7.88 \mathrm{E}-01$ & & \\
\hline $8.68 \mathrm{E}-01$ & $\mathrm{U}$ & 8.59E-01 & U & \\
\hline $2.61 \mathrm{E}-01$ & $\mathrm{U}$ & $7.88 \mathrm{E}-01$ & 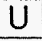 & \\
\hline NA & & NA & & \\
\hline $2.61 \mathrm{E}-01$ & $U$ & $7.88 \mathrm{E}-01$ & & \\
\hline
\end{tabular}




\section{Washington Closure Hanford}

Originator K. A. Anselm icaa

Project 300 Area Field Remediation

Subject Cleanup Verification 95\% UCL Calculations for 618-2 Shallow Zone/Deep Zone/Overburden and 618-2, 618-3, 618-8 Staging Pile and Decon Pad Footprint
CALCULATION SHEET

Date $\frac{11 / 01 / 06}{14655}$
Job No.

Calc. No. 0600X-CA-V0060

Checked J.M. Capron Q2m
Rev. No. 0

Date $\frac{11 / 01 / 06}{80 \text { of } 21}$

618-2 Deep Zone Sample Da

\begin{tabular}{|c|c|c|c|c|c|c|c|c|c|c|c|c|c|c|c|c|c|c|c|c|c|c|c|}
\hline \multirow{2}{*}{$\begin{array}{cc}2 & \text { Sampling } \\
3 & \text { Area }\end{array}$} & \multirow{2}{*}{$\begin{array}{l}\text { Sample } \\
\text { Number }\end{array}$} & \multirow{2}{*}{$\begin{array}{c}\text { Sample } \\
\text { Date }\end{array}$} & \multicolumn{3}{|c|}{ Plutonium-238 } & \multicolumn{3}{|c|}{ Plutonium-239/240 } & \multicolumn{3}{|c|}{ Plutonium-241 } & \multicolumn{3}{|c|}{ Strontium-90 } & \multicolumn{3}{|c|}{ Uranium-233/234 } & \multicolumn{3}{|c|}{ Uranium-235 } & \multicolumn{3}{|c|}{ Uranium-238 } \\
\hline & & & $\mathrm{pCi} / \mathrm{g}$ & $Q$ & MDA & $\mathrm{pCi} / \mathrm{g}$ & Q & MDA & $\mathrm{pCi} / \mathrm{g}$ & Q & MDA & $\mathrm{pCi} / \mathrm{g}$ & Q & MDA & $\mathrm{pCi} / \mathrm{g}$ & $Q$ & MDA & $\mathrm{pCi} / \mathrm{g}$ & Q & MDA & $\mathrm{pCi} / \mathrm{g}$ & a] & MDA \\
\hline A1 & J13DJ4 & $9 / 7 / 06$ & $9.8 \mathrm{E}-02$ & $U$ & $3.7 \mathrm{E}-01$ & $2.20 \mathrm{E}+00$ & & $3.7 \mathrm{E}-01$ & $7.07 \mathrm{E}+00$ & & $1.8 \mathrm{E}+01$ & $9.9 \mathrm{E}-02$ & $\mathrm{U}$ & $2.2 \mathrm{E}-01$ & $3.26 \mathrm{E}+00$ & & $2.2 \mathrm{E}-01$ & $1.02 \mathrm{E}-01$ & U & $2.6 \mathrm{E}-01$ & $2.87 \mathrm{E}+00$ & & $2.2 \mathrm{E}-01$ \\
\hline A3 & J13DJ2 & $9 / 7 / 06$ & $1.98 \mathrm{E}-01$ & U & $5.0 \mathrm{E}-01$ & $2.68 \mathrm{E}+01$ & & $5.0 \mathrm{E}-01$ & $2.08 \mathrm{E}+01$ & & $2.0 \mathrm{E}+01$ & $1.81 \mathrm{E}+00$ & & $2.2 \mathrm{E}-01$ & $5.53 \mathrm{E}+00$ & & $2.2 \mathrm{E}-01$ & $1.02 \mathrm{E}-01$ & $\mathrm{U}$ & $2.6 \mathrm{E}-01$ & $5.22 \mathrm{E}+00$ & & $2.2 \mathrm{E}-01$ \\
\hline A4 & J13DJ3 & $9 / 7 / 06$ & $1.50 E+00$ & & $4.8 \mathrm{E}-01$ & $9.06 \mathrm{E}+01$ & & $4.8 \mathrm{E}-01$ & $4.20 \mathrm{E}+01$ & & $2.6 \mathrm{E}+01$ & $1.75 \mathrm{E}+00$ & & 1.9E-01 & $1.61 E+02$ & & $4.8 \mathrm{E}-01$ & $3.35 \mathrm{E}+00$ & $U$ & $5.8 \mathrm{E}+00$ & $1.65 E+02$ & & $\begin{array}{l}4.6 \mathrm{E}-01 \\
20 \mathrm{C}-01\end{array}$ \\
\hline A2 & J13DJO & $9 / 7 / 06$ & $4.17 \mathrm{E}-01$ & $U$ & $5.3 \mathrm{E}-01$ & $5.16 \mathrm{E}+01$ & & $5.3 \mathrm{E}-01$ & $2.97 \mathrm{E}+01$ & & $2.9 \mathrm{E}+01$ & $1.16 \mathrm{E}+01$ & & $2.1 \mathrm{E}-01$ & $1.46 \mathrm{E}+01$ & & $2.9 \mathrm{E}-01$ & $7.76 \mathrm{E}-01$ & & $2.8 \mathrm{E}-01$ & $1.48 \mathrm{E}+01$ & & \\
\hline $\begin{array}{l}\text { Duplicate of } \\
\text { J13DJ0 }\end{array}$ & J13DJ1 & 9/7/06 & 4.15E-01 & $u$ & 4.5E-01 & $4.55 E+01$ & & $4.5 \mathrm{E}-01$ & $9.69 \mathrm{E}+00$ & u & $2.2 \mathrm{E}+01$ & $9.39 E+00$ & & $2.0 \mathrm{E}-01$ & $1.28 \mathrm{E}+01$ & & $3.0 \mathrm{E}-01$ & $7.61 \mathrm{E}-01$ & & $2.5 \mathrm{E}-01$ & $1.25 \mathrm{E}+01$ & & 2.6E-01 \\
\hline
\end{tabular}

11 Statistical Computation Input Data

\begin{tabular}{|c|c|c|c|c|c|c|c|c|c|}
\hline \multirow{2}{*}{$\begin{array}{c}\begin{array}{c}\text { Sampling } \\
\text { Area }\end{array} \\
A 1\end{array}$} & \multirow{2}{*}{$\begin{array}{c}\begin{array}{c}\text { Sample } \\
\text { Number }\end{array} \\
\mathrm{J} 13 \mathrm{D} J 4 \\
\end{array}$} & \multirow{2}{*}{$\begin{array}{c}\begin{array}{c}\text { Sample } \\
\text { Date }\end{array} \\
9 / 7 / 06 \\
\end{array}$} & $\begin{array}{l}\text { Plutonium-238 } \\
\mathrm{pCi} / \mathrm{g}\end{array}$ & $\begin{array}{l}\text { Plutonium-239/240 } \\
\text { pCi/g }\end{array}$ & $\begin{array}{l}\begin{array}{c}\text { Plutonium-241 } \\
\mathrm{pCi} / \mathrm{g}\end{array} \\
\end{array}$ & $\begin{array}{l}\text { Strontium-90 } \\
\mathrm{pCi/g}\end{array}$ & $\begin{array}{l}\text { Uranium-233/234 } \\
\text { pCi/g }\end{array}$ & \begin{tabular}{|c|}
$\begin{array}{c}\text { Uranium-235 } \\
\mathrm{pCi} / \mathrm{g}\end{array}$ \\
\end{tabular} & $\begin{array}{c}\text { Uranium-238 } \\
\text { pCi/g }\end{array}$ \\
\hline & & & \begin{tabular}{c|}
$9.8 \mathrm{E}-02$ \\
\end{tabular} & $2.20 \mathrm{E}+00$ & \begin{tabular}{|l|l|}
$7.07 E+00$ \\
\end{tabular} & \begin{tabular}{|c|}
$9.9 \mathrm{E}-02$ \\
\end{tabular} & $3.26 \mathrm{E}+00$ & $1.02 \mathrm{E}-01$ & $2.87 E+00$ \\
\hline A3 & J13DJ2 & $9 / 7 / 06$ & $1.98 \mathrm{E}-01$ & $2.68 \mathrm{E}+01$ & $2.08 \mathrm{E}+01$ & $1.81 E+00$ & $5.53 \mathrm{E}+00$ & $1.02 \mathrm{E}-01$ & $5.22 \mathrm{E}+00$ \\
\hline A4 & J13DJ3 & $9 / 7 / 06$ & $1.50 E+00$ & $9.06 \mathrm{E}+01$ & $4.20 \mathrm{E}+01$ & $1.75 \mathrm{E}+00$ & $1.61 E+02$ & $3.35 \mathrm{E}+00$ & $1.65 \mathrm{E}+02$ \\
\hline$A^{2}$ & J13DJO/J13DJ1 & $9 / 7 / 06$ & $4.16 \mathrm{E}-01$ & $4.86 E+01$ & $1.97 E+01$ & $1.05 E+01$ & $1.37 E+01$ & $7.69 \mathrm{E}-01$ & $1.37 \mathrm{E}+01$ \\
\hline
\end{tabular}

18

20 Statistical Computations

\begin{tabular}{|c|c|c|c|c|c|c|c|c|c|c|c|c|c|c|c|}
\hline \multirow{2}{*}{21[} & tour & Plutonium-2 & & Plutonium-2 & $239 / 240$ & Plutonium- & -241 & Strontium-s & & Uranium-233 & 234 & Uranium-23 & & Uranium-23 & \\
\hline & $95 \%$ UCL based on & $\begin{array}{l}\text { Radionuclid } \\
\text { nonparame }\end{array}$ & $\begin{array}{l}\text { data set. Use } \\
\text { tric z-statistic. }\end{array}$ & $\begin{array}{c}\text { Radionuclid } \\
\text { nonparam }\end{array}$ & $\begin{array}{l}\text { e data set. Use } \\
\text { tric z-statistic. }\end{array}$ & $\begin{array}{l}\text { Radionucl } \\
\text { Use nonp } \\
\text { sta }\end{array}$ & $\begin{array}{l}\text { lide data set. } \\
\text { oarametric } z \text { - } \\
\text { atistic. }\end{array}$ & $\begin{array}{c}\text { Radionuclic } \\
\text { nonparam }\end{array}$ & $\begin{array}{l}\text { data set. Use } \\
\text { tric } z \text {-statistic. }\end{array}$ & $\begin{array}{c}\text { Radionuclide } \\
\text { nonparame }\end{array}$ & $\begin{array}{l}\text { data set. Use } \\
\text { tric } z \text {-statistic. }\end{array}$ & $\begin{array}{l}\text { Radionuclid } \\
\text { nonparame }\end{array}$ & $\begin{array}{l}\text { data set. Use } \\
\text { tric } z \text {-statistic. }\end{array}$ & $\begin{array}{c}\text { Radionuclic } \\
\text { nonparam }\end{array}$ & $\begin{array}{l}\text { e data set. Use } \\
\text { tric z-statistic. }\end{array}$ \\
\hline 22 & & 4 & & 4 & & 4 & & 4 & & 4 & & 4 & & 4 & \\
\hline 24 & $\%<$ Detection limit & $\frac{4}{75 \%}$ & & $0 \%$ & & $0 \%$ & & $25 \%$ & & $0 \%$ & & $75 \%$ & & $0 \%$ & \\
\hline 25 & mean & $5.53 E-01$ & & $4.20 \mathrm{E}+01$ & & $2.24 E+01$ & & $3.5 \mathrm{E}+00$ & & $4.59 E+01$ & & $1.08 E+00$ & & $4.67 \mathrm{E}+01$ & \\
\hline 26 & st. dev. & $6.45 \mathrm{E}-01$ & & $3.75 E+01$ & & $1.45 \mathrm{E}+01$ & & $4.7 \mathrm{E}+00$ & & $7.69 \mathrm{E}+01$ & & $1.55 \mathrm{E}+00$ & & $7.90 \mathrm{E}+01$ & \\
\hline 27 & Z-statistic & 1.645 & & 1.645 & & 1.645 & & 1.645 & & 1.645 & & 1.645 & & 1.645 & \\
\hline 28 & $95 \%$ UCL on mean & $1.08 \mathrm{E}+00$ & & $7.29 E+01$ & & $3.43 E+01$ & & $7.4 \mathrm{E}+00$ & & $1.09 E+02$ & & $2.35 E+00$ & & $1.12 \mathrm{E}+02$ & \\
\hline 29 & max value & $1.50 \mathrm{E}+00$ & & $9.06 \mathrm{E}+01$ & & \begin{tabular}{|l|} 
\\
$4.20 \mathrm{E}+01$
\end{tabular} & & $1.2 \mathrm{E}+01$ & & $1.61 \mathrm{E}+02$ & & $3.35 E+00$ & & $1.65 \mathrm{E}+02$ & \\
\hline 30 & Statistical value & $1.08 \mathrm{E}+00$ & & $7.29 \mathrm{E}+01$ & & $3.43 \mathrm{E}+01$ & & $7.4 E+00$ & & $1.09 \mathrm{E}+02$ & & $2.35 E+00$ & & $1.12 E+02$ & \\
\hline 31 & Background & NA & & NA & & NA & & $\mathrm{NA}$ & & 1.1 & & 0.11 & & 1.1 & \\
\hline 32 & above background & $1.08 \mathrm{E}+00$ & & $7.29 E+01$ & & $3.43 E+01$ & & $7.4 \mathrm{E}+00$ & & $1.08 \mathrm{E}+02$ & & $2.24 \mathrm{E}+00$ & & $1.11 E+02$ & \\
\hline
\end{tabular}



and 618-2, 618-3, 618-8 Staging Pile and Decon Pad Footprint

\begin{tabular}{|c|c|c|c|c|c|c|c|c|c|c|c|c|c|c|c|c|c|c|c|c|c|c|}
\hline \multirow{3}{*}{\begin{tabular}{|c|} 
Sampling \\
Area \\
A1
\end{tabular}} & \multirow{2}{*}{$\begin{array}{l}\text { Sample } \\
\text { Number }\end{array}$} & \multirow{2}{*}{$\begin{array}{c}\text { Sample } \\
\text { Date }\end{array}$} & \multicolumn{3}{|c|}{ Arsenic } & \multicolumn{2}{|c|}{ Barium } & \multicolumn{3}{|c|}{ Cadmium } & \multicolumn{2}{|c|}{ Chromium } & \multicolumn{3}{|c|}{ Lead } & \multicolumn{3}{|c|}{ Selenium } & \multicolumn{2}{|r|}{ Tin } & \multicolumn{2}{|c|}{ Uranium } \\
\hline & & & $\mathrm{mg} / \mathrm{kg}$ & Q & PQL & $\mathrm{mg} / \mathrm{kg}$ & PQL & $\mathrm{g} / \mathrm{kg}$ & $Q$ & $\mathrm{PQL}$ & $\mathrm{mg} / \mathrm{kg}$ & $\begin{array}{l}2 \\
9 Q L\end{array}$ & $\mathrm{~g} / \mathrm{kg}$ & a & $\mathrm{PQL}$ & $\mathrm{mg} / \mathrm{kg}$ & 0 & PQL & $\mathrm{mg} / \mathrm{kg}$ & 0 & $\mathrm{mg} / \mathrm{kg}$ & \begin{tabular}{l|l} 
Q & PQL \\
\end{tabular} \\
\hline & J13DJ4 & $9 / 7 / 06$ & $1.4 \mathrm{E}+00$ & 4 & $1.1 E+00$ & $5.95 \mathrm{E}+01$ & $6 \mathrm{E}-02$ & $9 \mathrm{E}-02$ & U & $9 \mathrm{E}-02$ & $4.2 E+00$ & $2.1 E+00$ & $6.6 \mathrm{E}+00$ & & $3.5 \mathrm{E}-01$ & $7.8 \mathrm{E}-01$ & 11 & $7.6 E-01$ & $2.8 \mathrm{E}+00$ & $1.0 E+00$ & $4.54 \mathrm{E}+00$ & 7E-03 \\
\hline A3 & J1 & 917106 & $\frac{1.1 E+\infty 0}{15 E+00}$ & 0 & $\frac{1.1 \mathrm{E}+00}{1.15+0}$ & $5.85 t+01$ & & $\begin{array}{l}9 \mathrm{E}-02 \\
\mathrm{E}-0.02\end{array}$ & 0 & $9 E-02$ & $3.7 E+00$ & 2.1E+00 & $2.7 E+00$ & & $\begin{array}{l}3.5 \mathrm{E}-01 \\
25 \mathrm{~F}-1\end{array}$ & $\begin{array}{l}7.6 \mathrm{E}-01 \\
75 \mathrm{C}\end{array}$ & 0 & $\begin{array}{l}7.6 \mathrm{E} \\
7.5 \mathrm{E}\end{array}$ & $2.0 \mathrm{E}+00$ & | & $\mid$\begin{tabular}{|l|}
$1.37 \mathrm{E}+01$ \\
$501+\mathrm{E}$
\end{tabular} & $\begin{array}{l}7.2 E-02 \\
772501\end{array}$ \\
\hline & J13 & & $\frac{1.0 E+00}{1.1 E+00}$ & U & $\frac{1.1 E+00}{1.1 E+00}$ & $\frac{8.35+01}{6.35 E+01}$ & $\begin{array}{l}\frac{6 E-02}{6 \mathrm{E}-02} \\
\end{array}$ & $\begin{array}{l}\frac{9 E-02}{9 E-02} \\
9\end{array}$ & U & $\begin{array}{l}9 E-02 \\
9 E-02\end{array}$ & $\frac{0.1 E+00}{2.5 E+00}$ & $\frac{2.0 E-U}{2.0 E-01}$ & $\frac{3.8+00}{2.7 E+00}$ & & $\begin{array}{l}3.5-01 \\
3.5 \mathrm{E}-01\end{array}$ & $\begin{array}{l}.150-01 \\
7.6 \mathrm{E}-01\end{array}$ & u & $\frac{1.0 \mathrm{E}-\mathrm{W}}{7.6 \mathrm{E}-01}$ & $2.5 \mathrm{E}+00$ & $\frac{1.0 E+00}{1.0 E+00}$ & \begin{tabular}{|l|l|}
$3.57 \mathrm{E}+01$ \\
\end{tabular} & $\begin{array}{l}\frac{7.25-01}{7.2 \mathrm{E}-02} \\
\end{array}$ \\
\hline Duplicate of & J13DJ1 & $9 / 7 / 06$ & $1.1 \mathrm{E}+00$ & $u$ & $1.1 \mathrm{E}+00$ & $7.37 E+01$ & $6 \mathrm{E}-02$ & 9E-02 & u & $9 E-02$ & $4.4 \mathrm{E}+00$ & $2.1 E+00$ & $3.2 \mathrm{E}+00$ & & 3.5E-01 & 7.6E-01 & $u$ & 7.6E-01 & $2.9 \mathrm{E}+00$ & $1.0 E+00$ & $3.70 E+01$ & 7.2E-02 \\
\hline
\end{tabular}

11 Statistical Computation Input Data

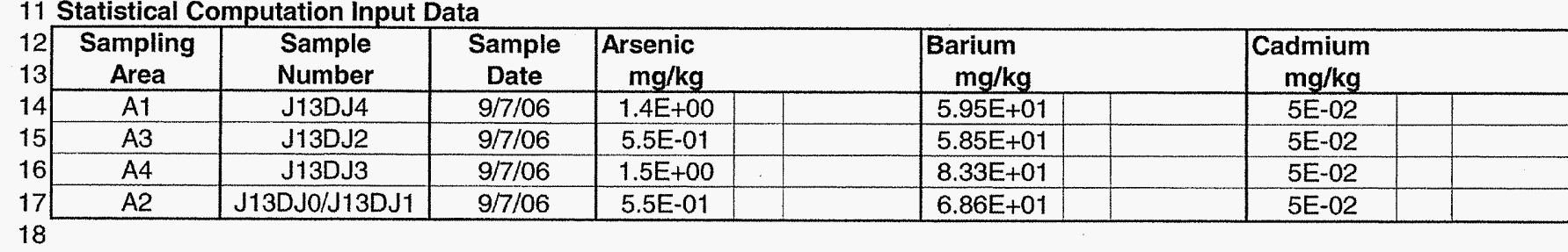

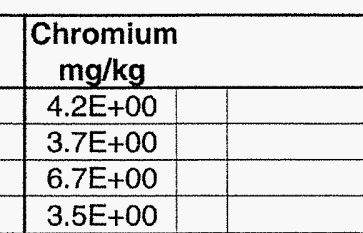
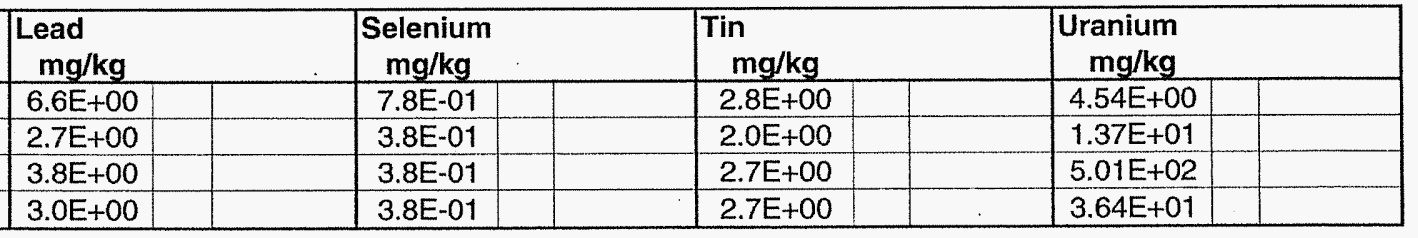

20 Statistical Computations

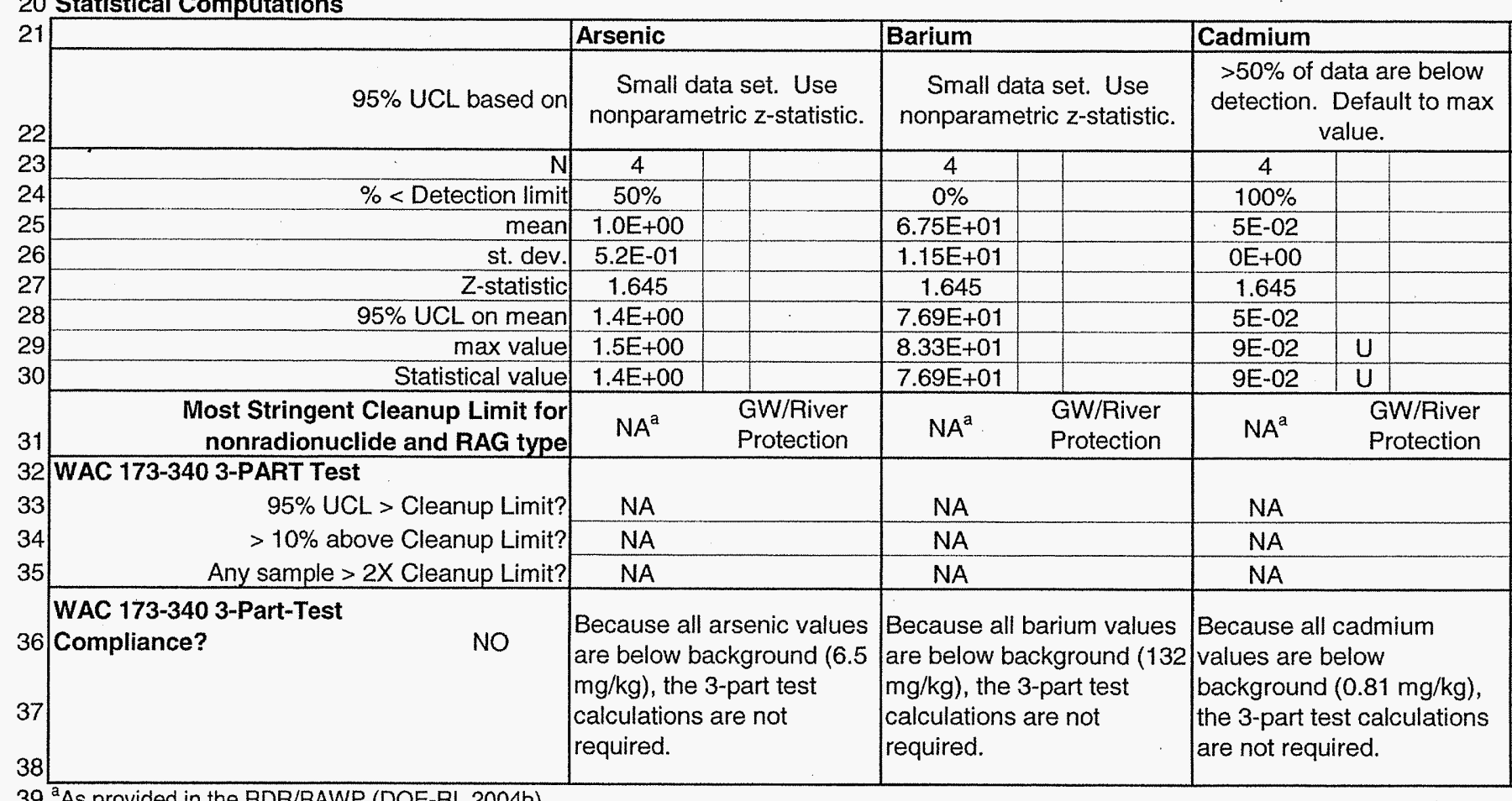

\begin{tabular}{|c|c|c|c|}
\hline \multirow{2}{*}{\multicolumn{2}{|c|}{$\begin{array}{l}\text { Chromium } \\
\text { Small data set. Use } \\
\text { nonparametric z-statistic. }\end{array}$}} & \multirow{2}{*}{\multicolumn{2}{|c|}{$\begin{array}{l}\text { Lead } \\
\text { Small data set. Use } \\
\text { nonparametric z-statistic. }\end{array}$}} \\
\hline & & & \\
\hline 4 & & 4 & \\
\hline $0 \%$ & & $0 \%$ & \\
\hline $\begin{array}{l}4.5 \mathrm{E}+00 \\
1.5 \mathrm{E}+00\end{array}$ & & \begin{tabular}{|l|l|}
$1.0 \mathrm{E}+00$ \\
\end{tabular} & \\
\hline 1.645 & & 1.645 & \\
\hline $5.7 E+00$ & & $5.5 \mathrm{E}+00$ & \\
\hline $6.7 E+00$ & & $\frac{6.6 E+00}{5-5 \Gamma}$ & \\
\hline $5.7 E+00$ & & $5.5 \mathrm{E}+00$ & \\
\hline \multicolumn{2}{|r|}{$\begin{array}{l}\text { GW/River } \\
\text { Protection }\end{array}$} & \multicolumn{2}{|r|}{$\begin{array}{l}\text { GW/River } \\
\text { Protection }\end{array}$} \\
\hline & \\
\hline \multicolumn{2}{|l|}{$\begin{array}{l}\text { NA } \\
\text { NA }\end{array}$} & \multirow{2}{*}{\multicolumn{2}{|c|}{$\begin{array}{l}\text { NA } \\
\text { NA }\end{array}$}} \\
\hline NA & & $\mathrm{NA}$ & \\
\hline \multicolumn{2}{|c|}{$\begin{array}{l}\text { Because all chromium } \\
\text { values are below } \\
\text { background }(18.5 \mathrm{mg} / \mathrm{kg}) \text {, } \\
\text { the 3-part test } \\
\text { calculations are are not } \\
\text { required. }\end{array}$} & \multicolumn{2}{|c|}{$\begin{array}{l}\text { Because all lead values } \\
\text { are below background } \\
\text { (10.2 } \mathrm{mg} / \mathrm{kg}) \text {, the 3-part } \\
\text { test calculations are not } \\
\text { required. }\end{array}$} \\
\hline
\end{tabular}

\begin{tabular}{|c|c|c|c|c|c|}
\hline \multirow{2}{*}{\multicolumn{2}{|c|}{$\begin{array}{l}\text { Selenium } \\
50 \% \text { of data are below } \\
\text { detection. Default to } \\
\text { max value. }\end{array}$}} & \multicolumn{2}{|c|}{ Tin } & \multicolumn{2}{|l|}{ Ura } \\
\hline & & \multicolumn{2}{|c|}{$\begin{array}{l}\text { Small data set. Use } \\
\text { nonparametric z-statistic. }\end{array}$} & \multicolumn{2}{|c|}{$\begin{array}{c}\text { Small data set. Use } \\
\text { nonparametric z-statistic }\end{array}$} \\
\hline 4 & & 4 & & & \\
\hline $75 \%$ & & $0 \%$ & & $0 \%$ & \\
\hline $4.8 \mathrm{E}-01$ & & $2.6 \mathrm{E}+00$ & & $1.39 \mathrm{E}+02$ & \\
\hline $2.0 \mathrm{E}-01$ & & \begin{tabular}{|l|}
$3.7 \mathrm{E}-01$ \\
\end{tabular} & & $2.42 \mathrm{E}+02$ & \\
\hline$\frac{2.0 L-01}{1.645}$ & & $\frac{0.15-01}{1.645}$ & & & \\
\hline$\frac{1.040}{6.4 \mathrm{E}-01}$ & & \begin{tabular}{|l}
1.940 \\
$2.9 \mathrm{E}+00$ \\
\end{tabular} & & $\frac{1.045}{3.38 \mathrm{E}+02}$ & \\
\hline$\frac{6.4 \mathrm{E}-0 !}{7.8 \mathrm{E}-01}$ & & \begin{tabular}{|l}
$.9 E+00$ \\
$2.9 \mathrm{E}+00$
\end{tabular} & & 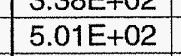 & \\
\hline $7.8 \mathrm{E}-01$ & & \begin{tabular}{|l}
$.9 \mathrm{E}+00$ \\
\end{tabular} & & $3.38 \mathrm{E}+02$ & \\
\hline $\mathrm{NA}^{\mathrm{a}}$ & $\begin{array}{l}\text { GW/River } \\
\text { Protection }\end{array}$ & & $\begin{array}{l}\text { GW/River } \\
\text { Protection }\end{array}$ & 385 & $\begin{array}{l}\text { GW/River } \\
\text { Protection }\end{array}$ \\
\hline \multicolumn{2}{|l|}{$\mathrm{NA}$} & \multicolumn{2}{|l|}{ NA } & \multicolumn{2}{|l|}{ NO } \\
\hline \multirow{2}{*}{\multicolumn{2}{|c|}{$\begin{array}{l}\mathrm{NA} \\
\mathrm{N}\end{array}$}} & \multirow{2}{*}{\multicolumn{2}{|c|}{ NA }} & \\
\hline & & & & & \\
\hline \multicolumn{2}{|c|}{$\begin{array}{l}\text { Because all selenium } \\
\text { values are at or below } \\
\text { background (0.78 } \\
\text { mg/kg), the 3-part test } \\
\text { calculations are not } \\
\text { required. }\end{array}$} & \multicolumn{2}{|c|}{$\begin{array}{l}\text { RESRAD predicts tin will } \\
\text { not reach GW within } \\
1,000 \text { years, per DOE-RL } \\
2004 \mathrm{~b} \text {. }\end{array}$} & \multicolumn{2}{|c|}{$\begin{array}{l}\text { Further assessment will } \\
\text { be performed because } \\
\text { uranium fails the 3-part } \\
\text { test. }\end{array}$} \\
\hline
\end{tabular}



and 618-2, 618-3,618-8 Staging Pile and Decon Pad Footprint

Calc. No. 0600X-CA-V0060

Checked J.M. Capron 2mC
Rev. No. $\frac{0}{\text { Date } \frac{0}{11 / 01 / 06}}$ Sheet No. $\frac{11 / 01 / 06}{10 \text { of } 21}$

\begin{tabular}{|c|c|c|c|c|c|c|c|c|c|c|c|c|c|c|c|c|c|c|c|c|c|c|c|c|c|c|}
\hline \multirow{2}{*}{\begin{tabular}{|c|}
$\begin{array}{c}\text { Sampling } \\
\text { Area }\end{array}$ \\
\end{tabular}} & \multirow{2}{*}{$\begin{array}{l}\text { Sample } \\
\text { Number }\end{array}$} & \multirow{2}{*}{$\begin{array}{c}\text { Sample } \\
\text { Date }\end{array}$} & \multicolumn{3}{|c|}{ Americium-241 } & \multicolumn{3}{|c|}{ Cesium-137 } & \multicolumn{3}{|c|}{ Cobalt-60 } & \multicolumn{3}{|c|}{ Europium-152 } & \multicolumn{3}{|c|}{ Europium-154 } & \multicolumn{3}{|c|}{ Europium-155 } & \multicolumn{3}{|c|}{ Nickel-63 } & \multicolumn{3}{|c|}{ Tritium } \\
\hline & & & $\mathrm{pCi} / \mathrm{g}$ & $Q$ & MDA & $\mathrm{pCi} / \mathrm{g}$ & $Q$ & MDA & $\mathrm{pCi} / \mathrm{g}$ & Q & MDA & $\mathrm{pCi} / \mathrm{g}$ & a. & MDA & $\mathrm{pCi/g}$ & Q & MDA & & Q & MDA & $\mathrm{pCi} / \mathrm{g}$ & 0 & MDA & $\mathrm{pCi} / \mathrm{g}$ & & MDA \\
\hline A1 & J13DF9 & $9 / 7 / 06$ & $-6.0 \mathrm{E}-03$ & $\bar{u}$ & $6.9 \mathrm{E}-02$ & $5.8 \mathrm{E}-02$ & & $3.9 \mathrm{E}-02$ & $3.3 \mathrm{E}-02$ & U & $3.3 \mathrm{E}-02$ & $9.0 \mathrm{E}-02$ & U⿺ & $9.0 \mathrm{E}-02$ & $1.1 \mathrm{E}-01$ & U⿺ & $1.1 \mathrm{E}-01$ & $8.9 \mathrm{E}-02$ & Uै & $8.9 \mathrm{E}-02$ & $-6.92 \mathrm{E}-01$ & J & $3.8 \mathrm{E}+00$ & $7.23 \mathrm{E}-01$ & $\bar{U}$ & $2.5 \mathrm{E}+00$ \\
\hline A3 & J13DH2 & $9 / 7 / 06$ & $3.5 \mathrm{E}-02$ & $\bar{U}$ & $6.2 \mathrm{E}-02$ & $3.6 \mathrm{E}-02$ & $u$ & $3.9 \mathrm{E}-02$ & $4.0 \mathrm{E}-02$ & U & $4.0 \mathrm{E}-02$ & $1.1 \mathrm{E}-01$ & U & $1.1 \mathrm{E}-01$ & $1.4 \mathrm{E}-01$ & U & $1.4 \mathrm{E}-01$ & 1.4E-01 & U & $1.4 \mathrm{E}-01$ & $1.02 E+00$ & & $4.1 E+00$ & $1.23 \mathrm{E}-01$ & 7 & $2.6 \mathrm{E}+00$ \\
\hline A4 & $\mathrm{J13DH3}$ & $9 / 7 / 06$ & $-1.2 \mathrm{E}-02$ & $\bar{U}$ & $8.2 \mathrm{E}-02$ & $4.0 \mathrm{E}-02$ & & $3.4 \mathrm{E}-02$ & $2.9 \mathrm{E}-02$ & U & $2.9 \mathrm{E}-02$ & $7.4 \mathrm{E}-02$ & $\mathrm{U}$ & $7.4 \mathrm{E}-02$ & $9.9 \mathrm{E}-02$ & U & $9.9 \mathrm{E}-02$ & $6.6 \mathrm{E}-02$ & $U$ & $6.6 \mathrm{E}-02$ & $2.43 \mathrm{E}-01$ & U & $4.3 \mathrm{E}+00$ & $9.62 \mathrm{E}-\mathrm{C}$ & $U$ & $2.4 E+00$ \\
\hline $\mathrm{A} 2$ & J13DHO & $9 / 7 / 06$ & $2.0 \mathrm{E}-02$ & U & $6.5 \mathrm{E}-02$ & $4.2 \mathrm{E}-02$ & $U$ & $4.2 \mathrm{E}-02$ & $3.8 \mathrm{E}-02$ & $u$ & $3.8 \mathrm{E}-02$ & $9.3 \mathrm{E}-02$ & $U$ & $9.3 \mathrm{E}-02$ & $1.2 \mathrm{E}-01$ & U & $1.2 \mathrm{E}-01$ & $8.4 \mathrm{E}-02$ & U & $8.4 \mathrm{E}-02$ & \begin{tabular}{|l|}
$-7.17 E-01$ \\
\end{tabular} & U & $3.7 E+00$ & 3.49E-01 & $\underline{U}$ & $2.6 \mathrm{E}+00$ \\
\hline $\begin{array}{c}\text { Duplicate of } \\
J 13 \mathrm{DHO}\end{array}$ & J13DH1 & $9 / 7 / 06$ & $-5.0 \mathrm{E}-03$ & u & 7.0E-02 & $4.8 \mathrm{E}-02$ & & $4.8 \mathrm{E}-02$ & 4.9E-02 & $\mathrm{u}$ & $4.9 \mathrm{E}-02$ & $1.2 E-01$ & $u$ & $1.2 \mathrm{E}-01$ & $1.6 \mathrm{E}-01$ & $u$ & $1.6 \mathrm{E}-01$ & $1.1 \mathrm{E}-01$ & $u$ & $1.1 \mathrm{E}-01$ & $-1.15 \mathrm{E}+00$ & u & $4.0 E+00$ & $5.62 E-01$ & U & $2.6 \mathrm{E}+00$ \\
\hline
\end{tabular}
9

10 Statistical Computation Input Data

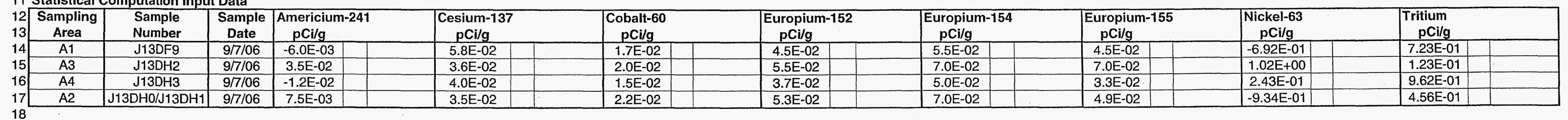

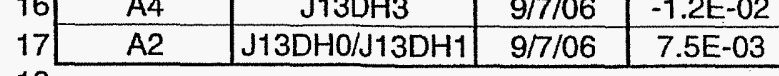

20 Statistical Computations

\begin{tabular}{|c|c|c|c|c|c|c|c|c|c|}
\hline \multirow{3}{*}{$\begin{array}{l}21 \\
22 \\
23\end{array}$} & & \multicolumn{3}{|c|}{ Americium-241 } & \multicolumn{2}{|c|}{ Cesium-137 } & \multicolumn{3}{|l|}{ Cobalt-60 } \\
\hline & \multirow[t]{2}{*}{$95 \%$ UCL based on } & \multicolumn{3}{|c|}{$\begin{array}{l}\text { Radionuclide data set. } \\
\text { Use nonparametric z- } \\
\text { statistic. }\end{array}$} & \multicolumn{2}{|c|}{$\begin{array}{l}\text { Radionuclide data set. Use } \\
\text { nonparametric } z \text {-statistic. }\end{array}$} & \multicolumn{3}{|c|}{$\begin{array}{l}\text { Radionuclide data set } \\
\text { Use nonparametric } \mathrm{z} \text { - } \\
\text { statistic. }\end{array}$} \\
\hline & & 4 & & & 4 & & & & \\
\hline 24 & $\%<$ Detection limit & $100 \%$ & & & $25 \%$ & & $100 \%$ & & \\
\hline 25 & mean & $6.1 \mathrm{E}-03$ & & & $4.2 \mathrm{E}-02$ & & $1.8 \mathrm{E}-02$ & & \\
\hline 26 & st. dev. & $2.1 \mathrm{E}-02$ & & & $1.1 \mathrm{E}-02$ & & $3.3 \mathrm{E}-03$ & & \\
\hline 27 & Z-statistic & 1.645 & & & 1.645 & & 1.645 & & \\
\hline & $95 \%$ UCL on mean & $2.3 \mathrm{E}-02$ & & & $5.1 \mathrm{E}-02$ & & $2.1 \mathrm{E}-02$ & & \\
\hline 29 & max value & $3.5 \mathrm{E}-02$ & $u$ & & $5.8 \mathrm{E}-02$ & & $4.9 \mathrm{E}-02$ & $\mathrm{u}$ & \\
\hline 30 & Statistical value & $2.3 \mathrm{E}-02$ & $\mathrm{U}$ & & $5.1 \mathrm{E}-02$ & & $2.1 \mathrm{E}-02$ & $\bar{U}$ & \\
\hline 31] & Background & NA & & & 1.1 & & 0.008 & & \\
\hline 32 & Statistical value above background & $2.3 \mathrm{E}-02$ & $u$ & & $0(<\mathrm{BG})$ & & $1.3 \mathrm{E}-02$ & $\mathrm{U}$ & \\
\hline
\end{tabular}

\begin{tabular}{l|l} 
& Europium-155 \\
\hline
\end{tabular}

Nickel-63

Tritium

\begin{tabular}{|c|c|c|}
\hline $\begin{array}{l}\text { adionuclide data set. } \\
\text { Ise nonparametric z- }\end{array}$ & $\begin{array}{l}\text { Radionuclide data set. } \\
\text { Use nonparametric } z\end{array}$ & $\begin{array}{l}\text { Radionuclide data set. } \\
\text { Use nonparametric } \mathrm{z}-\end{array}$ \\
\hline
\end{tabular}

statistic.
s.

\begin{tabular}{c|c}
$100 \%$ & \\
\hline
\end{tabular}

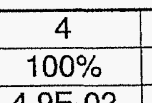

\begin{tabular}{c|c}
$1.0 \mathrm{E}-02$ \\
1.645 \\
$7.0 \mathrm{E}$
\end{tabular}

\begin{tabular}{c|c|}
\hline & $4.5 \mathrm{E}-02$ \\
\hline 1.045 \\
\hline $7.0 \mathrm{E}-02$ & 1.645 \\
\hline & $1.6 \mathrm{E}-01$ \\
\hline
\end{tabular}

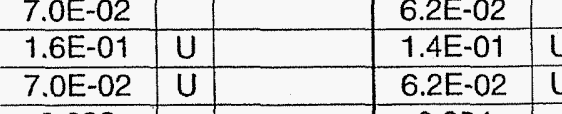

\begin{tabular}{c|c|c|}
\hline 0.033 & \\
\hline $37 \mathrm{E}-02$ & \\
\hline
\end{tabular}

\begin{tabular}{|c|c|c|c|c|}
\hline $1.5 \mathrm{E}-02$ & & & $8.98 \mathrm{E}-01$ & \\
\hline 1.645 & & & 1.645 & \\
\hline $6.2 \mathrm{E}-02$ & & & $6.48 \mathrm{E}-01$ & \\
\hline $1.4 \mathrm{E}-01$ & $\mathrm{U}$ & & $1.02 \mathrm{E}+00$ & $\mathrm{U}$ \\
\hline $6.2 \mathrm{E}-02$ & $\mathrm{U}$ & & $6.48 \mathrm{E}-01$ & $\mathrm{U}$ \\
\hline 0.054 & & & $\mathrm{NA}$ & \\
\hline $7.7 \mathrm{E}-03$ & $\mathrm{U}$ & & $6.48 \mathrm{E}-01$ & $\mathrm{U}$ \\
\hline
\end{tabular}

\begin{tabular}{c|l|l}
\hline 4 & \\
\hline $100 \%$ & & \\
$9.06 \mathrm{E}-02$ & & \\
$.98 \mathrm{E}-01$ & & \\
\hline 1.645 & & \\
$.48 \mathrm{E}-01$ & & \\
$.02 \mathrm{E}+00$ & $\mathrm{U}$ & \\
\hline $.48 \mathrm{E}-01$ & $\mathrm{U}$ & \\
\hline NA & & \\
\hline $48 \mathrm{E}-01$ & $\mathrm{U}$ & \\
\hline
\end{tabular}

ametric $\mathrm{z}$ -

$100 \%$

\begin{tabular}{l}
$5.66 \mathrm{E}-01$ \\
$3.61 \mathrm{E}-01$ \\
\hline 1.045
\end{tabular}

\begin{tabular}{c|c}
$3.61 \mathrm{E}-01$ \\
\hline 1.645 \\
\hline $8.62 E-01$
\end{tabular}

\begin{tabular}{|l|l}
\hline $8.62 E-01$ & \\
\hline $9.62 E-01$ & $U$
\end{tabular} \begin{tabular}{ll}
$9.62 \mathrm{E}-01$ & $\mathrm{U}$ \\
\hline $8.62 \mathrm{E}-01$ & $\mathrm{U}$
\end{tabular} \begin{tabular}{|c|c|c|}
\hline NA & & \\
$8.62 \mathrm{E}-01$ & $\mathrm{U}$ & \\
\hline
\end{tabular} 


\section{Washington Closure Hanford}

Originator K. A. Anselm Ka a

Field Remediation

Subject Cleanup Verification $95 \%$ UCL Calculations for 618-2 Shallow Zone/Deep Zone/Overburden and 618-2, 618-3, 618-8 Staging Pile and Decon Pad Footprint
CALCULATION SHEET

Date 11/01/06

Job No. 14655
Calc. No. $0600 \mathrm{X}-\mathrm{CA}-\mathrm{V} 0060$

Checked J.M. Capron Q2mC

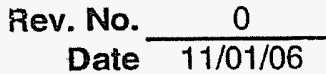
Sheet No. $\frac{110106}{11 \text { of } 21}$

\begin{tabular}{|c|c|c|c|c|c|c|c|c|c|c|c|c|c|c|c|c|c|c|c|c|c|c|c|}
\hline \multirow{2}{*}{$\begin{array}{c}\text { Sampling } \\
\text { Area }\end{array}$} & \multirow{2}{*}{$\begin{array}{l}\text { Sample } \\
\text { Number }\end{array}$} & \multirow{2}{*}{$\begin{array}{c}\text { Sample } \\
\text { Date }\end{array}$} & \multicolumn{3}{|c|}{ Plutonium-238 } & \multicolumn{3}{|c|}{ Plutonium-239/240 } & \multirow{2}{*}{\multicolumn{3}{|c|}{ Plutonium-241 }} & \multicolumn{3}{|c|}{ Strontium-90 } & \multirow{2}{*}{\multicolumn{3}{|c|}{ Uranium-233/234 }} & \multicolumn{3}{|c|}{ Uranium-235 } & \multicolumn{3}{|c|}{ Uranium-238 } \\
\hline & & & $\mathrm{pCi} / \mathrm{g}$ & a & MDA & $\mathrm{pCi} / \mathrm{g}$ & Q & MDA & & Q & MDA & $\mathrm{pCi} / \mathrm{g}$ & Q & MDA & $\mathrm{pCi} / \mathrm{g}$ & & & $\mathrm{pCi} / \mathrm{g}$ & Q & MDA & $\mathrm{pCi} / \mathrm{g}$ & & MDA \\
\hline A1 & J13DF9 & $9 / 7 / 06$ & $-6.2 \mathrm{E}-02$ & U & $4.7 \mathrm{E}-$ & & $\bar{U}$ & $4.7 \mathrm{E}$ & $7.94 \mathrm{E}+00$ & U & $2.2 \mathrm{E}+01$ & $5.1 \mathrm{E}-02$ & $u$ & $2.6 \mathrm{E}-01$ & $5.42 \mathrm{E}-01$ & & $1.9 \mathrm{E}-01$ & $3.0 \mathrm{E}-02$ & $U$ & & $4.44 \mathrm{E}-01$ & & $1.9 \mathrm{E}-01$ \\
\hline A3 & & $9 / 7 / 06$ & 0 & $u$ & $4.9 \mathrm{E}$ & $6.5 \mathrm{E}-02$ & $\mathrm{U}$ & & $-5.23 E+00$ & $u$ & & & $\mathrm{U}$ & & & & & 32500 & U & & 01 & & \\
\hline A4 & & $9 / 7 / 06$ & 0 & $u$ & $5.2 \mathrm{E}$ & 0 & u & $5.2 \mathrm{E}-01$ & \begin{tabular}{|l|}
$-4.35 E+00$ \\
\end{tabular} & $u$ & $2.5 \mathrm{E}+01$ & $-2.4 \mathrm{E}-02$ & $\mathrm{U}$ & 2.4 & & & & & $\bar{U}$ & & & & 2.1E-01 \\
\hline$A^{2}$ & $\mathrm{~J} 13 \mathrm{DHO}$ & $9 / 7 / 06$ & $1.26 \mathrm{E}-01$ & $U$ & $4.8 \mathrm{E}-01$ & 0 & U & $4.8 \mathrm{E}-01$ & $4.63 \mathrm{E}+00$ & $u$ & $2.4 \mathrm{E}+01$ & $-1.2 \mathrm{E}-02$ & $u$ & $2.5 \mathrm{E}-01$ & 6. & & $2.1 \mathrm{E}$ & $9.7 \mathrm{E}-02$ & $u$ & $2.5 \mathrm{E}-01$ & $5.10 \mathrm{E}-01$ & & $2.1 \mathrm{E}-01$ \\
\hline (ח & J13DH1 & $9 / 7 / 06$ & & u & $5.0 \mathrm{E}-01$ & $1.97 \mathrm{E}-01$ & $\mathrm{u}$ & $5.0 \mathrm{E}-01$ & $4.82 E+00$ & & $2.8 \mathrm{E}+01$ & $-9.7 E-02$ & $u$ & $2.5 \mathrm{E}-01$ & $5.37 \mathrm{E}-01$ & & $2.1 \mathrm{E}-01$ & 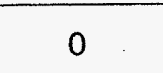 & $u$ & $2.5 \mathrm{E}-01$ & $5.91 \mathrm{E}-01$ & & $2.1 \mathrm{E}-01$ \\
\hline
\end{tabular}
9

11 Statistical Computation Input Data

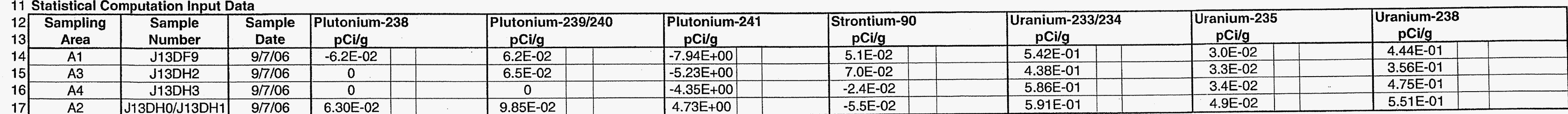

17

20 Statistical Computations

\begin{tabular}{|c|c|c|c|c|c|c|c|c|c|c|}
\hline \multirow{2}{*}{22} & \multicolumn{4}{|c|}{ Plutonium-238 } & \multicolumn{3}{|c|}{ Plutonium-239/240 } & \multicolumn{3}{|c|}{ Plutonium-241 } \\
\hline & $95 \%$ UCL based on & $\begin{array}{c}\text { Radionuclide } \\
\text { nonparamet }\end{array}$ & $\begin{array}{l}\text { da } \\
\text { tric }\end{array}$ & $\begin{array}{l}\text { ta set. Use } \\
\text { z-statistic. }\end{array}$ & $\begin{array}{c}\text { Radionuclid } \\
\text { nonparame }\end{array}$ & $\begin{array}{l}\text { e da } \\
\text { tric }\end{array}$ & $\begin{array}{l}\text { ata set. Use } \\
\text { z-statistic. }\end{array}$ & $\begin{array}{r}\text { Radionucl } \\
\text { Use nonp } \\
\text { sta }\end{array}$ & $\begin{array}{l}\text { lide } \\
\text { arara } \\
\text { atisti }\end{array}$ & $\begin{array}{l}\text { data set. } \\
\text { metric z- } \\
\text { c. }\end{array}$ \\
\hline 23 & & 4 & & & 4 & & & 4 & & \\
\hline 24 & $\%<$ Detection limit & $100 \%$ & & & $100 \%$ & & & $100 \%$ & & \\
\hline 25 & mean & $2.5 \mathrm{E}-04$ & & & $5.6 \mathrm{E}-02$ & & & $-3.20 E+00$ & & \\
\hline $26-$ & st. dev. & $5.1 \mathrm{E}-02$ & & & $4.1 \mathrm{E}-02$ & & & $5.50 \mathrm{E}+00$ & & \\
\hline 27 & Z-statistic & 1.645 & & & 1.645 & & & 1.645 & & \\
\hline 28 & $95 \%$ UCL on mean & $4.2 \mathrm{E}-02$ & & & $9.0 \mathrm{E}-02$ & & & $1.32 \mathrm{E}+00$ & & \\
\hline 29 & max value & $1.26 \mathrm{E}-01$ & $\mathrm{U}$ & & $1.97 \mathrm{E}-01$ & $U$ & & $4.82 E+00$ & $\bar{U}$ & \\
\hline $30 †$ & Statistical value & $4.2 \mathrm{E}-02$ & U & & $9.0 \mathrm{E}-02$ & $U$ & & $1.32 E+00$ & $\mathrm{U}$ & \\
\hline 31) & Background & 0.004 & & & 0.025 & & & NA & & \\
\hline & & & & & $6.5 \mathrm{E}-02$ & & & & & \\
\hline
\end{tabular}

Strontium-90

Uranium-233/234

Uranium-235

Uranium-238

Radionuclide data set Use Radionuclide data set. Use Radionuclide data set. Use Radionuclide data set. Use nonparametric z-statistic. nonparametric $z$-statistic.

nonparametric $z$-statistic

Radionuclide data set. Use

\begin{tabular}{l|l|l|l} 
& \\
\hline
\end{tabular}

\begin{tabular}{c}
$\mathrm{NA}$ \\
$1.32 \mathrm{E}+00$ \\
\hline
\end{tabular}

\begin{tabular}{|c|l|l|c|c|c|}
\hline 4 & & & 4 & & \\
\hline $1.0 \%$ & & & $0 \%$ & & \\
\hline $1.1 \mathrm{E}-02$ & & & $5.39 \mathrm{E}-01$ & & \\
\hline $5.9 \mathrm{E}-02$ & & & $7.09 \mathrm{E}-02$ & & \\
\hline 1.645 & & & 1.645 & & \\
\hline $6.0 \mathrm{E}-02$ & & & $5.97 \mathrm{E}-01$ & & \\
\hline $7.0 \mathrm{E}-02$ & $\mathrm{U}$ & & $6.44 \mathrm{E}-01$ & & \\
\hline $6.0 \mathrm{E}-02$ & $\mathrm{U}$ & & $5.97 \mathrm{E}-01$ & & \\
\hline 0.18 & & & 1.1 & & \\
\hline 0.18 & & $0(\mathrm{BG})$ & \\
\hline
\end{tabular}

\begin{tabular}{|c|c|c|}
\hline 4 & & \\
\hline $100 \%$ & & \\
\hline $3.6 \mathrm{E}-02$ & & \\
\hline $8.3 \mathrm{E}-03$ & & \\
\hline 1.645 & & \\
\hline $4.3 \mathrm{E}-02$ & & \\
\hline $9.7 \mathrm{E}-02$ & $U$ & \\
\hline $4.3 \mathrm{E}-02$ & $U$ & \\
\hline 0.11 & & \\
\hline
\end{tabular}

\begin{tabular}{|c|c|c|}
\hline 4 & \\
\hline $0 \%$ & & \\
\hline
\end{tabular}

$0 \%$

$8.05 \mathrm{E}-02$

1.645

5.23E-01

5.23E-01

\begin{tabular}{l|l|l|l|l}
0.18 & & 1.1 \\
\hline $0(<B G)$ & & & 0
\end{tabular}

0.11 


\begin{tabular}{|c|c|c|c|c|c|c|c|c|c|c|c|c|c|c|c|c|c|c|c|c|c|}
\hline \multirow{2}{*}{\begin{tabular}{|c|c|}
2 & Sampling \\
3 & Area \\
\end{tabular}} & \multirow{2}{*}{$\begin{array}{l}\text { Sample } \\
\text { Number } \\
\end{array}$} & \multirow{2}{*}{$\begin{array}{c}\text { Sample } \\
\text { Date }\end{array}$} & \multicolumn{2}{|c|}{ Arsenic } & \multicolumn{2}{|c|}{ Barium } & \multicolumn{3}{|c|}{ Cadmium } & \multicolumn{2}{|c|}{ Chromium } & \multicolumn{2}{|r|}{ Lead } & \multicolumn{3}{|c|}{ Selenium } & \multicolumn{3}{|c|}{$\operatorname{Tin}$} & \multicolumn{2}{|c|}{ Uranium } \\
\hline & & & $\mathrm{mg} / \mathrm{kg}$ & $\mathrm{PQL}$ & $\mathrm{mg} / \mathrm{kg}$ & \begin{tabular}{|l|l|}
$\mathbf{Q}$ & $\mathrm{PQL}$ \\
\end{tabular} & $\mathrm{mg} / \mathrm{kg}$ & 10 & $P Q L$ & $\mathrm{mg} / \mathrm{kg}$ & $\begin{array}{l}Q \quad P Q L \\
\end{array}$ & $\mathrm{mg} / \mathrm{kg}$ & \begin{tabular}{l|l}
0 & $P Q L$ \\
\end{tabular} & $\mathrm{mg} / \mathrm{kg}$ & & $P Q L$ & $\mathrm{mg} / \mathrm{kg}$ & 0 & PQL & $\mathrm{mg} / \mathrm{kg}$ & \begin{tabular}{l|l} 
Q & $P Q L$ \\
\end{tabular} \\
\hline $\begin{array}{l}A 1 \\
A 3\end{array}$ & $\frac{J 13 D F 9}{\sqrt{130 H 2}}$ & $\begin{array}{l}9 / 7 / 166 \\
9 / 106\end{array}$ & $\frac{2.7 \mathrm{E}+00}{1.8 \mathrm{E}+00}$ & $\frac{1.2 E+00}{1.1 E+00}$ & \begin{tabular}{|l|}
$6.42 E+01$ \\
$7.47 E+01$ \\
\end{tabular} & $\frac{6 \mathrm{E}-02}{6 \mathrm{E}-02}$ & $\begin{array}{l}9 \mathrm{E}-02 \\
\mathrm{E}-\mathrm{-}-02 \\
\end{array}$ & $\frac{u}{u}$ & $\begin{array}{l}9 \mathrm{E}-02 \\
\mathrm{gE}-02 \\
\end{array}$ & \begin{tabular}{|l|}
$\frac{6.1 \mathrm{E}+00}{5.1 \mathrm{E}+00}$ \\
\end{tabular} & $\frac{2.2 .2-01}{2.0 E-01}$ & $\left|\begin{array}{|l}\mid 4.1 \mathrm{E}+00 \\
4.3 \mathrm{E}+00\end{array}\right|$ & $\frac{3.8-01}{3.5 E-01}$ & \begin{tabular}{|l|}
$8.2 E-01$ \\
$1.0 E+00$
\end{tabular} \mid & U & $\frac{8.2-0-01}{7.6 \mathrm{E}-01}$ & \begin{tabular}{|l|}
$2.7 \mathrm{E}+00$ \\
$.6 \mathrm{E}+00$
\end{tabular} & & $\left|\begin{array}{|l}1.1 \mathrm{E}+00 \\
1.0 \mathrm{E}+00\end{array}\right|$ & \begin{tabular}{|l|}
$1.14 E+00$ \\
$9.15 E-01$ \\
\end{tabular} & $\begin{array}{l}7-03 \\
7 E-03 \\
\end{array}$ \\
\hline A4 & $\mathrm{J} 13 \mathrm{DH} 3$ & $9 / 7 / 106$ & $2.4 E+00$ & $1.1 \mathrm{E}+00$ & $6.29 \mathrm{E}+01$ & $6 \mathrm{E}-02$ & $9 \mathrm{E}-02$ & u & 9E-02 & \begin{tabular}{|l|}
$0.1 L+00$ \\
$5.2 E+00$
\end{tabular} & & \begin{tabular}{|l|}
$4.0 E+00$ \\
$3.4 E+00$ \\
\end{tabular} & 3.5E-01 & \begin{tabular}{|l|}
$1.0 c+00$ \\
$.5 \mathrm{E}-01$ \\
\end{tabular} & U & $7.5 \mathrm{E}-01$ & $2.0 \mathrm{E}+00$ & & & $8.72 \mathrm{E}-01$ & $7 \mathrm{E}-03$ \\
\hline $\mathrm{A} 2$ & $\mathrm{~J} 13 \mathrm{DHO}$ & $9 / 7 / 106$ & $1.5 \mathrm{E}+00$ & $1.1 \mathrm{E}+00$ & $6.22 E+01$ & $6 \mathrm{E}-02$ & $9 \mathrm{E}-02$ & U & 9E-02 & $5.3 \mathrm{E}+00$ & $2.0 E-01$ & $3.5 \mathrm{E}+00$ & $3.5 E-01$ & $7.6 \mathrm{E}-01$ & $\bar{u}$ & $7.6 \mathrm{E}-01$ & $1.0 E+00$ & $u$ & $1.0 \mathrm{E}+00$ & $9.72 \mathrm{E}-01$ & $7 E-03$ \\
\hline $\begin{array}{l}\text { Duplicate of } \\
\text { J133HO }\end{array}$ & $\mathrm{J} 13 \mathrm{DH} 1$ & 9/7/06 & $1.2 E+00$ & $1.1 \mathrm{E}+00$ & $6.35 \mathrm{E}+01$ & $6 \mathrm{E}-02$ & 9E-02 & $u$ & 9E-02 & $6.6 \mathrm{E}+00$ & $2.0 \mathrm{E}-01$ & $4.3 E+00$ & $3.5 \mathrm{E}-01$ & $7.5 E-01$ & u & $7.5 \mathrm{E}-01$ & $2.2 \mathrm{E}+00$ & & $1.0 E+00$ & 1E-01 & 03 \\
\hline
\end{tabular}
9
10

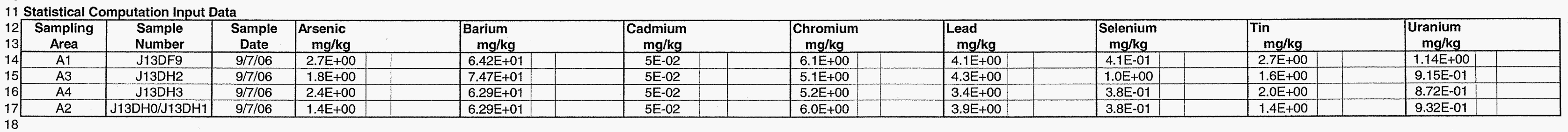
18
19

\begin{tabular}{|c|c|c|c|c|c|c|c|c|c|c|c|c|c|c|c|c|c|}
\hline \multirow{4}{*}{$\begin{array}{l}22 \\
23 \\
24\end{array}$} & \multirow[b]{2}{*}{$95 \%$ UCL based on } & \multirow{2}{*}{\multicolumn{2}{|c|}{\begin{tabular}{|l|} 
Arsenic \\
Small data set. Use \\
nonparametric z-statistic.
\end{tabular}}} & \multirow{2}{*}{\multicolumn{2}{|c|}{\begin{tabular}{|l} 
Barium \\
Small data set. Use \\
nonparametric z-statistic.
\end{tabular}}} & \multirow{2}{*}{\multicolumn{2}{|c|}{$\begin{array}{l}\text { Cadmium } \\
>50 \% \text { of data are below } \\
\text { detection. Default to max } \\
\text { value. }\end{array}$}} & \multirow{2}{*}{\multicolumn{2}{|c|}{\begin{tabular}{|l} 
Chromium \\
Small data set. Use \\
nonparametric z-statistic.
\end{tabular}}} & \multirow{2}{*}{\multicolumn{2}{|c|}{$\begin{array}{l}\text { Lead } \\
\text { Small data set. Use } \\
\text { nonparametric z-statistic. }\end{array}$}} & \multirow{2}{*}{\multicolumn{2}{|c|}{\begin{tabular}{|l|} 
Selenium \\
$50 \%$ of data are below \\
detection. Default to \\
max value.
\end{tabular}}} & \multirow{2}{*}{\multicolumn{2}{|c|}{$\begin{array}{l}\text { Tin } \\
\text { Small data set. Use } \\
\text { nonparametric z-statistic. }\end{array}$}} & \multirow{2}{*}{\multicolumn{2}{|c|}{$\begin{array}{l}\text { Uranium } \\
\text { Small data set. Use } \\
\text { nonparametric z-statistic. }\end{array}$}} \\
\hline & & & & & & & & & & & & & & & & & \\
\hline & & 4 & & 4 & & 4 & & 4 & & 4 & & 4 & & 4 & & 4 & \\
\hline & $\%<$ Detection limit & $0 \%$ & & $0 \%$ & & $100 \%$ & & $0 \%$ & & & & & & & & & \\
\hline & mean & $2.1 \mathrm{E}+00$ & & $6.62 \mathrm{E}+01$ & & $5 E-02$ & & $5.6 \mathrm{E}+00$ & & $3.9 \mathrm{E}+00$ & & $5.4 \mathrm{E}-01$ & & $1.9 \mathrm{E}+00$ & & $9.65 E-01$ & \\
\hline 26 & st. dev. & $6.0 \mathrm{E}-01$ & & $5.73 \mathrm{E}+00$ & & $0 E+00$ & & $5.1 \mathrm{E}-01$ & & $3.9 \mathrm{E}-01$ & & $3.1 \mathrm{E}-01$ & & $5.9 \mathrm{E}-01$ & & $1.20 \mathrm{E}-01$ & \\
\hline $27 \mid$ & Z-statistic & 1.645 & & 1.645 & & 1.645 & & 1.645 & & 1.645 & & 1.645 & & 1.645 & & 1.645 & \\
\hline 28 & $95 \%$ UCL on mean & $2.6 \mathrm{E}+00$ & & $7.09 \mathrm{E}+01$ & & $5 \mathrm{E}-02$ & & $6.0 \mathrm{E}+00$ & & $4.2 E+00$ & & $7.9 \mathrm{E}-01$ & & $2.4 E+00$ & & $1.06 \mathrm{E}+00$ & \\
\hline tot & $\begin{array}{r}\text { max value } \\
\text { Strtiotinas }\end{array}$ & \begin{tabular}{|l|l|}
$2.7 E+00$ \\
$265+00$
\end{tabular} & & \begin{tabular}{|l|}
$7.47 \mathrm{E}+01$ \\
7005.01 \\
\end{tabular} & & $9 \mathrm{E}-02$ & $u$ & $6.6 \mathrm{E}+00$ & & $4.3 \mathrm{E}+00$ & & $1.0 E+00$ & & $2.7 \mathrm{E}+00$ & & $\frac{1.14 \mathrm{E}+00}{1.00}$ & \\
\hline 31] & $\begin{array}{c}\text { Statistical value } \\
\begin{array}{c}\text { Most Stringent Cleanup Limit for } \\
\text { nonradionuclide and RAG type }\end{array}\end{array}$ & \begin{tabular}{|c|}
$2.6 \mathrm{E}+00$ \\
20
\end{tabular} & $\begin{array}{c}\text { Direct } \\
\text { Exposure }\end{array}$ & \begin{tabular}{|l|}
$7.09 E+01$ \\
4,900 \\
\end{tabular} & $\begin{array}{c}\text { Direct } \\
\text { Exposure }\end{array}$ & $\begin{array}{c}9 \mathrm{EE}-02 \\
139\end{array}$ & $\begin{array}{l}\text { U } \\
\substack{\text { Direct } \\
\text { Exposure }}\end{array}$ & $\frac{6.0 E+00}{1,000,000}$ & $\begin{array}{l}\text { Direct } \\
\text { Exposure }\end{array}$ & \begin{tabular}{|c|}
$4.2 E+00$ \\
1,000
\end{tabular} & $\begin{array}{c}\text { Direct } \\
\text { Exposure }\end{array}$ & $\frac{1.0 E+00}{17,500}$ & $\begin{array}{c}\text { Direct } \\
\text { Exposure }\end{array}$ & \begin{tabular}{|l|}
$2.4 \mathrm{E}+00$ \\
$1,000,000$ \\
\end{tabular} & $\begin{array}{c}\text { Direct } \\
\text { Exposure }\end{array}$ & $\frac{1.06 E+00}{505}$ & $\begin{array}{c}\text { Direct } \\
\text { Exposure }\end{array}$ \\
\hline & WAC 173-340 3-PART Test & & & & & 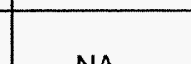 & & N & & $\mathrm{F}$ & & N & & 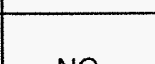 & & & \\
\hline 35 & Any sample $>2 X$ Cleanup Limit? & $\mathrm{NA}$ & & $\mathrm{NA}$ & & NA & & NA & & NA & & NO & & NO & & NO & \\
\hline $\begin{array}{l}36 \\
37\end{array}$ & $\begin{array}{l}\text { EXCESS RISK EVALUATION: } \\
\text { WAC 173-340 Noncarcinogenic Cleanup: }\end{array}$ & NA & & NA & & NA & & NA & & NA & & 17,500 & & $1,000,000$ & & 505 & \\
\hline & Hazard quotient for each nonradionuclide: & NA & & NA & & NA & & NA & & NA & & $5,7 E-05$ & & $2.4 \mathrm{E}-06$ & & $2.1 E-03$ & \\
\hline 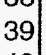 & WAC 173-340 Carcinogenic Cleanup: & NA & & NA & & NA & & NA & & NA & & NA & & NA & & NA & \\
\hline & Risk for each carcinogenic nonradionuclide: & NA & & NA & & NA & & NA & & NA & & NA & & NA & & NA & \\
\hline & $\begin{array}{l}\text { WAC 173-340 3-Part-Test } \\
\text { Compliance? } \\
\text { Nonrad noncarcinogenic } \\
\text { index sum: } \\
\text { Nonrad carcinogenic risk: }\end{array}$ & $\begin{array}{l}\text { Because all } \\
\text { are below ba } \\
\text { mg/kg), the ? } \\
\text { excess risk } \\
\text { not required. }\end{array}$ & $\begin{array}{l}\text { rsenic values } \\
\text { kground }(6.5 \\
\text { part test and } \\
\text { alculations are }\end{array}$ & $\begin{array}{l}\text { Because all } \\
\text { are below bo } \\
\text { mg/kg), the : } \\
\text { excess risk } \\
\text { not required. }\end{array}$ & $\begin{array}{l}\text { arium values } \\
\text { ckground ( } 132 \\
\text {-part test and } \\
\text { alculations are }\end{array}$ & $\begin{array}{l}\text { Because all ce } \\
\text { values are bel } \\
\text { background (C } \\
\text { the 3-part test } \\
\text { risk calculatio } \\
\text { required. }\end{array}$ & $\begin{array}{l}\text { admium } \\
\text { low } \\
0.81 \mathrm{mg} / \mathrm{kg}) \\
\text { it and excess } \\
\text { ons are not. }\end{array}$ & \begin{tabular}{|l} 
Because all \\
values are \\
background \\
the 3-part te \\
excess risk \\
are not requ
\end{tabular} & $\begin{array}{l}\text { Shromium } \\
\text { laow } \\
(18.5 \mathrm{mg} / \mathrm{kg}) \\
\text { tand } \\
\text { and } \\
\text { acultions } \\
\text { red. }\end{array}$ & $\begin{array}{l}\text { Because al } \\
\text { are below } \mathrm{k} \\
(10.2 \mathrm{mg} / \mathrm{k} \\
\text { test and ex } \\
\text { calculation } \\
\text { required. }\end{array}$ & $\begin{array}{l}\text { lead values } \\
\text { ackground } \\
\text { chthe } 3 \text {-part } \\
\text { ess risk } \\
\text { are not }\end{array}$ & & & & & & \\
\hline
\end{tabular}


1 618-2, 618-3, and 618-8 Staging Pile/Decon Pad Footprint Sample Data

\begin{tabular}{|c|c|c|c|c|c|c|c|c|c|c|c|c|c|c|c|c|c|c|c|c|c|c|c|c|}
\hline \multirow{2}{*}{$\begin{array}{c}\text { Sampling } \\
\text { Area }\end{array}$} & \multirow{2}{*}{$\begin{array}{l}\text { Sample } \\
\text { Number } \\
\end{array}$} & \multirow{2}{*}{\begin{tabular}{|c|}
$\begin{array}{c}\text { Sample } \\
\text { Date }\end{array}$ \\
\end{tabular}} & \multirow{2}{*}{\multicolumn{3}{|c|}{ Americium-241 }} & \multirow{2}{*}{\multicolumn{3}{|c|}{ Cesium-137 }} & \multicolumn{3}{|c|}{ Cobalt-60 } & \multicolumn{3}{|c|}{ Europium-152 } & \multicolumn{3}{|c|}{ Europium-154 } & \multicolumn{3}{|c|}{ Europium-155 } & \multicolumn{2}{|c|}{ Nickel-63 } & \multicolumn{2}{|c|}{ Trifium } \\
\hline & & & pcilg & & & $\mathrm{pCi} / \mathrm{g}$ & & MDA & pCilg & & MDA & $\mathrm{pCi} / \mathrm{g}$ & & MDA & $\mathrm{pCi} / \mathrm{g}$ & & & $\mathrm{pCi} / \mathrm{g}$ & & MDA & 0 & MDA & $\mathrm{pCi} / \mathrm{g}$ & \begin{tabular}{l|l} 
& MDA \\
\end{tabular} \\
\hline B5 & & & $-1.5 \mathrm{E}-02$ & $\begin{array}{l}u \\
u\end{array}$ & 6.1E-02 & 4.6E-02 & & 4.6E-02 & $4.0 \mathrm{E}-02$ & & $4.0 \mathrm{E}-02$ & $1.1 \mathrm{E}-01$ & $\bar{U}$ & 1.1.E -01 & 1.4E-01 & $u$ & 1.4EE-01 & $1.0 \mathrm{E}-01$ & & $1.0 \mathrm{E}-$ & 3.56E-01 & & -02 & $4 E+\infty 0$ \\
\hline $\begin{array}{l}B 6 \\
B 7\end{array}$ & $3 \mathrm{D} 60$ & $\frac{9 / 6 / 06}{90 / 6 / 66}$ & $4.5 \mathrm{E}-02$ & U & $6.4 \mathrm{E}-02$ & 4.4E- 02 & & $\begin{array}{l}4.4 E-02 \\
30.02\end{array}$ & $4.3 E-02$ & & $4.3 \mathrm{E}-02$ & 1.1E-01 & 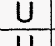 & & & 5 & & 1.0E-01 & & 1.0 & 78 & & & \\
\hline & & & \begin{tabular}{|c|}
$-6.0 \mathrm{E}-03$ \\
$31 \mathrm{~F}-02$
\end{tabular} & $\frac{U}{u}$ & $\frac{6.1 \mathrm{E}-02}{48 \mathrm{E}-\mathrm{O}-2}$ & $\begin{array}{l}3.0 E-02 \\
27 F-02\end{array}$ & & $\begin{array}{l}3.0 E-02 \\
277-02\end{array}$ & $\frac{2.6 E-02}{32 F-0 ?}$ & u & $\frac{2.6 E-02}{320-02}$ & $\frac{7.0 \mathrm{E}-02}{661 \mathrm{~F}-02}$ & $\mathrm{U}$ & $\frac{7.0 \mathrm{E}-02}{6.02}$ & $\frac{8.9 \mathrm{E}-02}{10 \mathrm{C}}$ & IIt & 8.96 & $\frac{6.3 \mathrm{E}-02}{8.4 \mathrm{~F}-0 ?}$ & & $845=0 ?$ & $102 F+0$ & & $\begin{array}{l}.61 E-01 \\
128 \mathrm{E}-01\end{array}$ & $2.7 E+000$ \\
\hline $\mathrm{Cg}$ & & $6 / 0$ & \begin{tabular}{|l|}
$3.1 \mathrm{~L}-0 \mathrm{~L}$ \\
$4.0 \mathrm{E}-03$
\end{tabular} & u & & $\frac{2 . E-02}{2.0 E-02}$ & & $2.7 \mathrm{t}$ & $\frac{3.2 .2-02}{2.0 E-02}$ & & \begin{tabular}{|l|}
$3.2 \mathrm{E}-02$ \\
$2.0 \mathrm{E}-02$ \\
\end{tabular} & $\frac{6.11}{4.5 \mathrm{E}}$ & & & & & & $\frac{8.4 \mathrm{E}-02}{44.8 \mathrm{E}-02}$ & & \begin{tabular}{|l|}
$8.4 \mathrm{E}-02$ \\
$4.8 \mathrm{E}-02$ \\
\end{tabular} & $\frac{1.02 E+00}{5.47 F-01}$ & $\frac{3.7 E+00}{3.7 E+00}$ & $\frac{1.28 E-01}{0}$ & $\frac{2.4 E+00}{2.3 E+00}$ \\
\hline $\mathrm{C1}$ & & & $8.18 \mathrm{E}-\mathrm{|}$ & & & $9.5 \mathrm{E}-02$ & & 2.4 & $2.2 E-02$ & & 2.21 & \begin{tabular}{|l|}
$5.6 \mathrm{E}-02$ \\
\end{tabular} & 5 & & & 0 & & $\frac{.8 E-02}{8 E-02}$ & & & & & & $2.3 E+00$ \\
\hline & & & & & & & & & & & & & 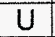 & & & u & & & & & & & & $= \pm+\infty$ \\
\hline & & & & & & $5.5 \mathrm{E}-\mathrm{C}$ & & & & & $5.4 \mathrm{E}$ & 1.5E-01 & & & 2.1 & 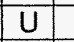 & & 2.11 & & $2.1 \mathrm{E}$ & $6.74 E$ & & $6.95 E-01$ & \begin{tabular}{l|l} 
UJ & $2.3 \mathrm{E}+00$
\end{tabular} \\
\hline & & & & & & & & & & & & & & & & & & & & & 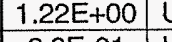 & 3.7 & & $2.5 E+00$ \\
\hline D. & & & 4. & & & & & & & & & $5 E_{-}$ & & & $8.0 \mathrm{E}-\mathrm{C}$ & & & & & & & & & \\
\hline $\mathrm{Df}$ & & & & & & & & & & & & 1E & & & & & & & & & & & & $25 \mathrm{E}$ \\
\hline A1 & & & $\begin{array}{l}-2,0 \mathrm{E}-02 \\
8,0 \mathrm{E}-03\end{array}$ & & & & & & & & & & & & & & & & & & & & & $= \pm 0$ \\
\hline Ai & & & & & & & & & & & & & & & & $\bar{U}$ & & & & & & & & \\
\hline $\mathrm{A}^{4}$ & & $\pi$ & \begin{tabular}{|l|l|}
$1.1 \mathrm{E}-02$ \\
$.1550 ?$
\end{tabular} & $\bar{u}$ & $\frac{6.0 E-02}{55002}$ & $4.5 \mathrm{E}-$ & & $4.5 \mathrm{E}$ & $4.6 \mathrm{E}-02$ & & $\begin{array}{l}4.6 E-02 \\
27 E_{-02}\end{array}$ & $\begin{array}{l}1.2 \mathrm{E}-01 \\
77502 \\
\end{array}$ & $\bar{u}$ & $1.2 E-01$ & 1.7E-01 & $u$ & $\begin{array}{l}1.7 E-01 \\
85020\end{array}$ & $1.8 \mathrm{E}-01$ & & $\begin{array}{ll}1.8 \mathrm{E}-01 \\
6 . \mathrm{E}-0\end{array}$ & -01 & $\begin{array}{l}4.1 E+C \\
37 F+0\end{array}$ & $\begin{array}{l}1.12 E+00 \\
1118 E+00\end{array}$ & $24 E+0 c$ \\
\hline A & J1: & $9 / 6 / 0$ & $-1.5 \mathrm{E}-02$ & 0 & $5.5 E-0.6$ & 3.1E-02 & U & $3.1 \mathrm{E}-02$ & $2.7 E-02$ & 0 & $2.76-02$ & 7.7t-02 & 0 & 1. $1 \mathrm{E}-02$ & $8.2 \mathrm{E}-02$ & U & $8.2 E-0.6$ & $6.6 \mathrm{E}-02$ & 0 & $0.0 E-06$ & $0.05 E-01 / 2$ & $3 . / f+c$ & 102 & $4 E+O C$ \\
\hline & J13D59 & $9 / 6 / 06$ & 2.2E-02 & $u$ & $.9 E-02$ & 4.6E-02 & u & $4.6 \mathrm{E}-02$ & $4.2 \mathrm{E}-02$ & & $4.2 E-02$ & 1.1E-01 & 10 & 1.1E-01 & $1.5 \mathrm{E}-01$ & u & $1.5 \mathrm{E}-01$ & $9.9 \mathrm{E}-02$ & & $9.9 \mathrm{E}-02$ & | 2.15E-01 | L & 4.4E+00 & $1.11 \mathrm{E}+00$ & \begin{tabular}{l|l} 
UU & $2.3 E+00$
\end{tabular} \\
\hline
\end{tabular}

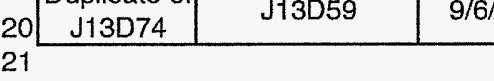

22 Statistical Computation Input Data

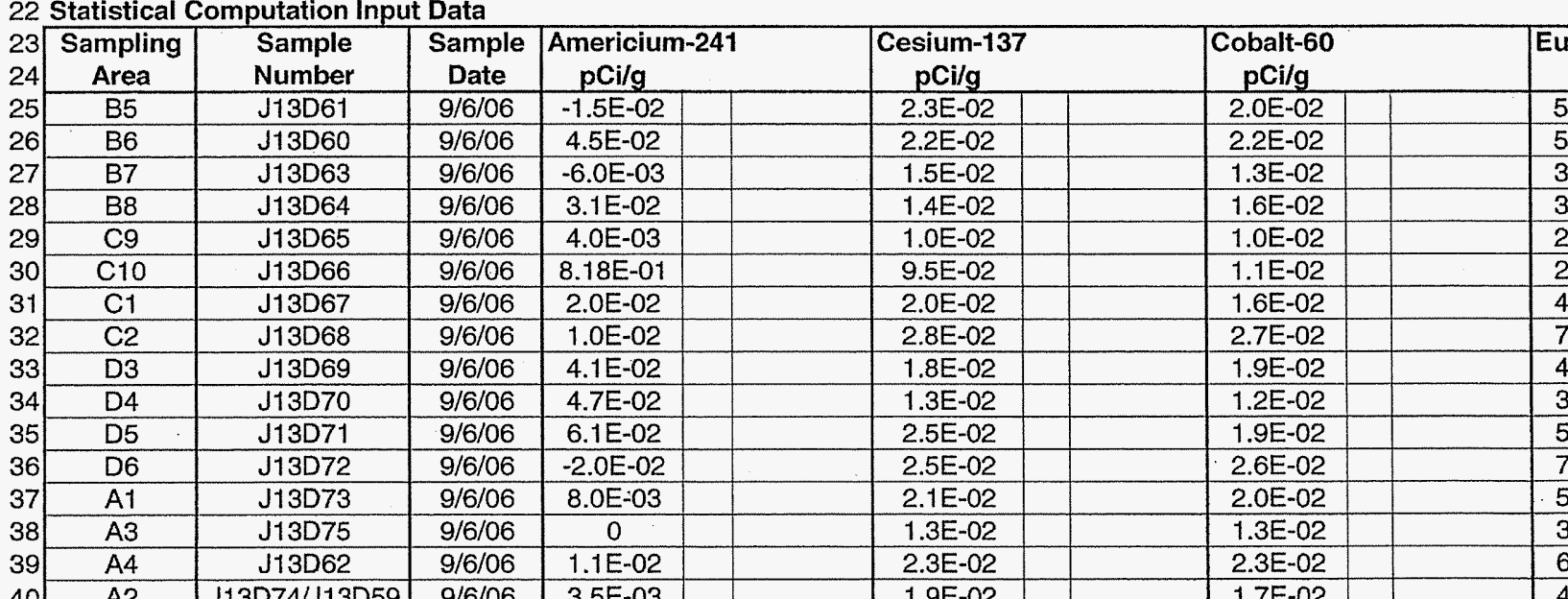

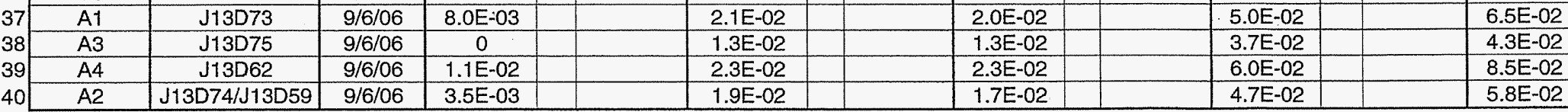

42 Statistical Computations

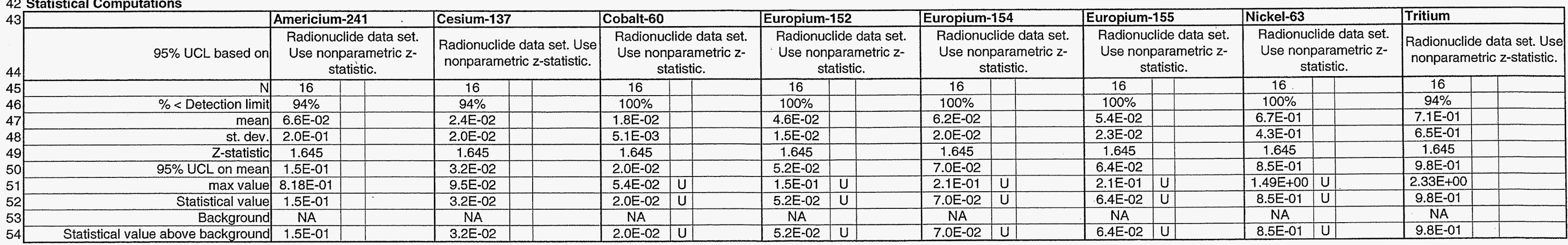


Washington Closure Hanford

Originator K.A. Anselm KCQO

C. A Ans

for 618-2 Shallow Zone/Deep Zone/Overburden.

CALCULATION SHEET

Date $\frac{11 / 16 / 06}{14655}$

Checked J. M. Capron gero

Rev. No. $\frac{1}{11 / 16 / 106}$

Dheet No. $\frac{11 / 16 / 06}{14 \text { of } 21}$

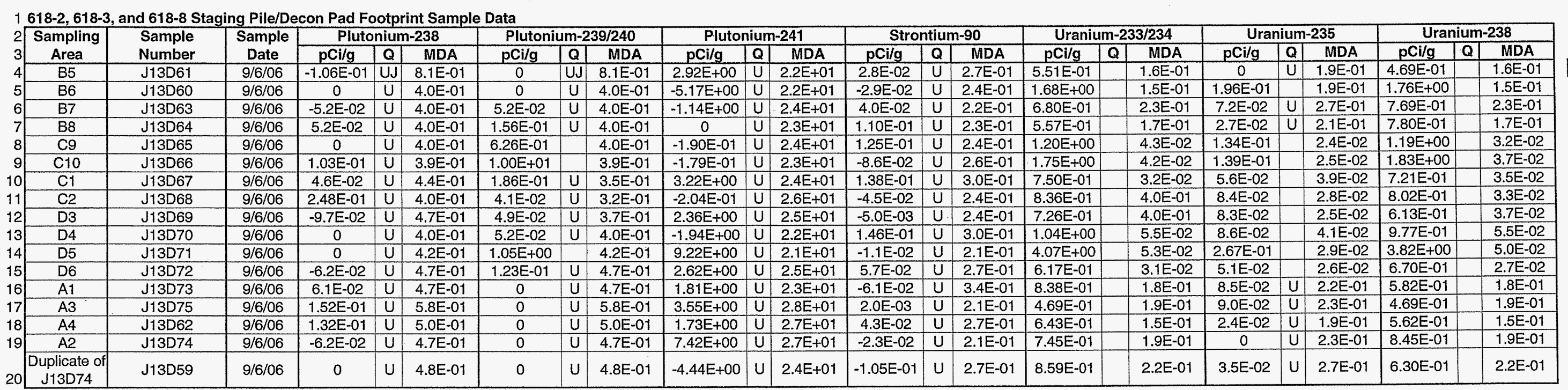

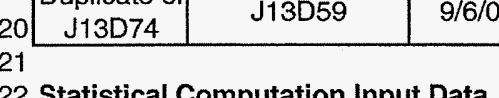

2 Statistical Computation Input Data
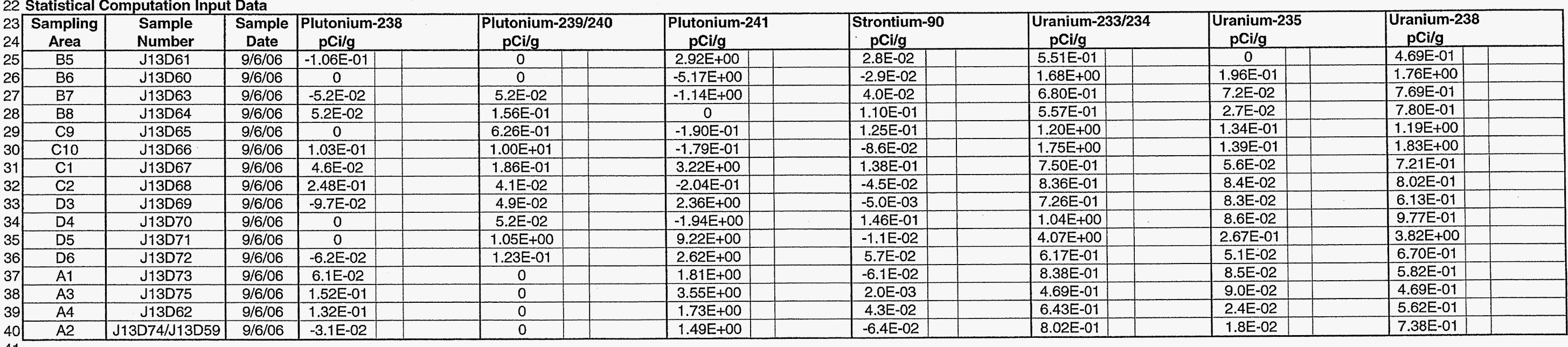

42 Statistical Computations

\begin{tabular}{|c|c|c|c|c|c|}
\hline \multirow{2}{*}{\multicolumn{2}{|c|}{ 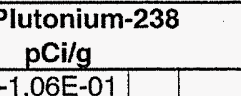 }} & \multicolumn{2}{|c|}{$\begin{array}{l}\text { Plutonium-239/240 } \\
\text { pCi/g }\end{array}$} & \multicolumn{2}{|c|}{$\begin{array}{l}\text { Plutonium-241 } \\
\text { pCC/g }\end{array}$} \\
\hline & & & & & \\
\hline & & 0 & & \begin{tabular}{|l|}
$-5.17 E+00$ \\
\end{tabular} & \\
\hline$\frac{-02}{-02}$ & & $\frac{5.2 E-02}{156-01}$ & & $|-1.14 E+00|$ & \\
\hline & & $6.26 \mathrm{E}-01$ & & \begin{tabular}{|c|} 
\\
$-1.90 E-01$
\end{tabular} & \\
\hline & & $1.00 \mathrm{E}+01$ & & \begin{tabular}{|l|}
$-1.79 E-01$ \\
\end{tabular} & \\
\hline & & $1.86 \mathrm{E}-01$ & & \begin{tabular}{|l|}
$3.22 \mathrm{E}+00$ \\
\end{tabular} & \\
\hline-01 & & $\begin{array}{l}4.1 E-02 \\
10503\end{array}$ & & \begin{tabular}{|c|}
$-2.04 \mathrm{E}-01$ \\
$23 \mathrm{E}+00$ \\
\end{tabular} & \\
\hline & & $\begin{array}{l}4.9 E-02 \\
502-03\end{array}$ & & $\mid$\begin{tabular}{|c|}
$2.36 \mathrm{E}+00$ \\
$-194 \mathrm{~A}+00$
\end{tabular} & \\
\hline & & $\frac{5.2 E-02}{105 E+00}$ & & $\mid$\begin{tabular}{|c|}
$-1.94 E+00$ \\
$9.22 E+00$
\end{tabular} & \\
\hline$=02$ & & $\frac{1.05 \mathrm{E}+01}{1.23 \mathrm{E}-01}$ & & $\mid$ & \\
\hline & & 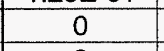 & & $1.81 E+00$ & \\
\hline & & & & $3.55 E+00$ & \\
\hline $\begin{array}{ll}E-01 \\
=02\end{array}$ & & 0 & & $1.73 \mathrm{E}+00$ & \\
\hline
\end{tabular}

\begin{tabular}{|l|l|}
\hline $4.3 E-02$ & $6.43 E-01$ \\
\hline
\end{tabular} $5.62 \mathrm{E}-01$

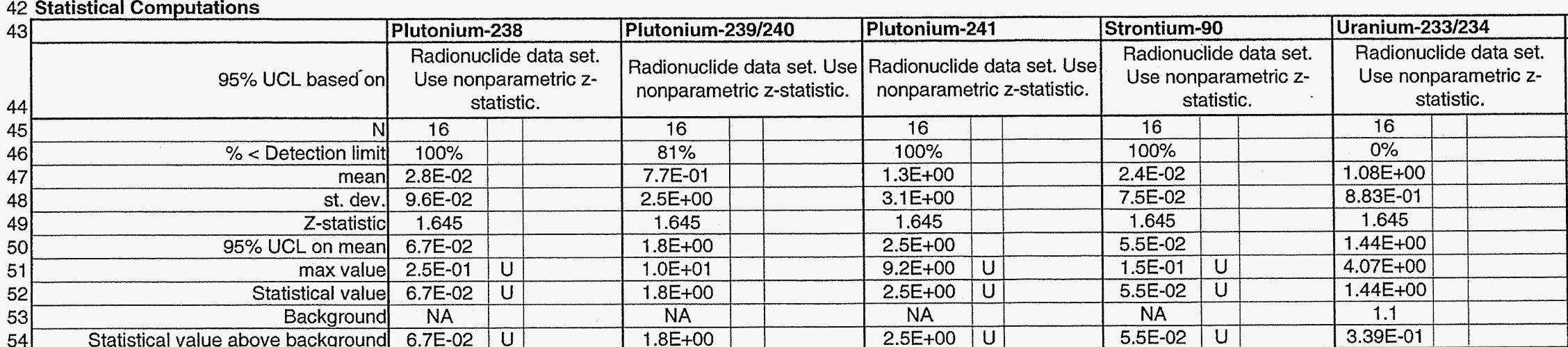

\begin{tabular}{|c|c|c|c|}
\hline \multirow{2}{*}{\multicolumn{2}{|c|}{$\begin{array}{l}\text { Uranium-235 } \\
\text { Radionuclide data set. } \\
\text { Use nonparametric z- } \\
\text { statatistic. } \\
\end{array}$}} & \multirow{2}{*}{\multicolumn{2}{|c|}{\begin{tabular}{|c|} 
Uranium-238 \\
Radionuclide data set \\
Use nonparametric $z-$ \\
statistic. \\
\end{tabular}}} \\
\hline & & & \\
\hline & & & \\
\hline 8E-02 & & & \\
\hline & & 8.44 & \\
\hline & & & \\
\hline $\mid=-01$ & & $\begin{array}{l}\frac{1.395}{3.82 E} \\
\end{array}$ & \\
\hline$\frac{2 E-01}{211}$ & & \begin{tabular}{|r|}
$1.39 E+00$ \\
11
\end{tabular} & \\
\hline$\frac{0.11}{6 \mathrm{E}-0}$ & & $\frac{1.1}{1.94 \mathrm{E}-01}$ & \\
\hline
\end{tabular}



and 618-2, 618-3, 618-8 Staging Pile and Decon Pad Footprint

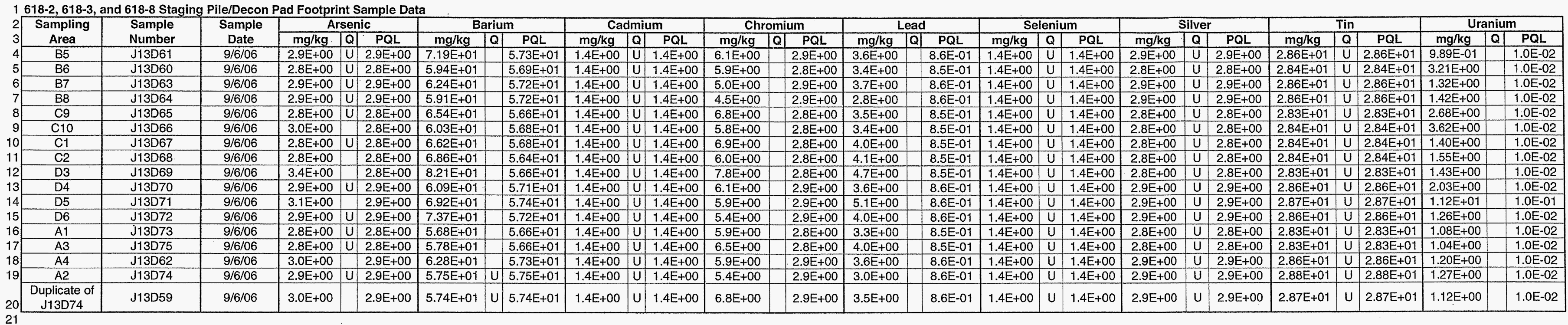
21

(tatistical Computation Input Data

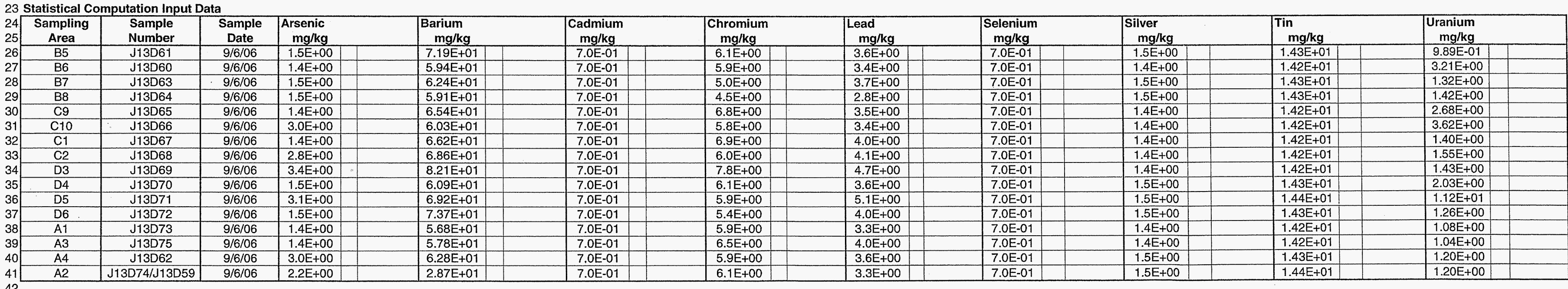


Washington Closure Hanford

Originator K. A. Anselm lcaa

$\begin{array}{cc}\text { Project } 300 \text { Area Field Remediation } & \text { Date } 11 / 01 / 06 \\ \text { Job No. } 14655 & \end{array}$

and 618-2, 618-3,618-8 Staging Pile and Decon Pad Folitow Zone/Deep Zone//Vverburden,

and 618-8 Staging Pile/Decon Pad Footprint Statistical Computations

\section{\begin{tabular}{l}
$1618-2,618-3$, and 618-8 Staging Pile/Decon Pad Footprint Statistical Computations \\
2 \\
\hline Arsenic
\end{tabular}}
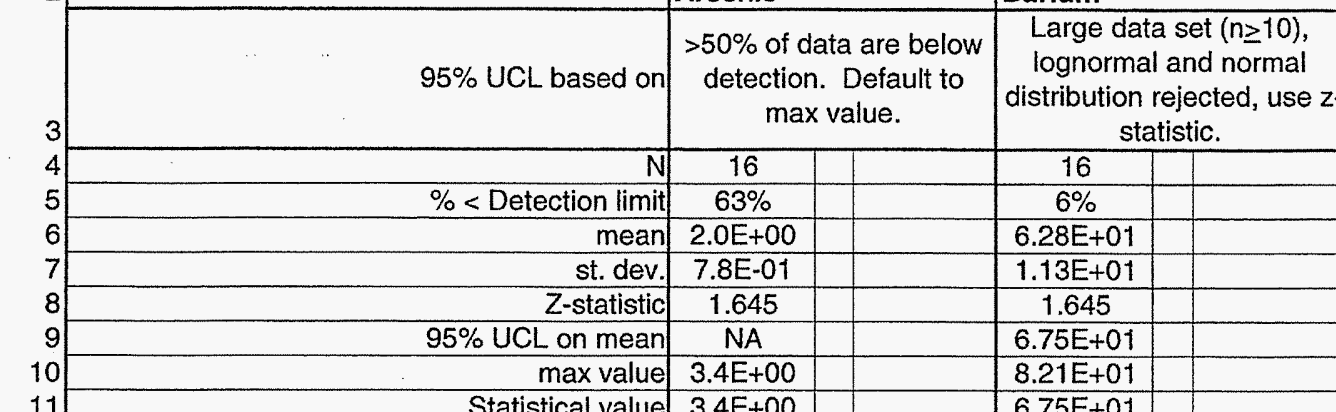

$>50 \%$ of data are below
detection. Default to Large data set $(n \geq 10)$

\begin{tabular}{l|l} 
statistic. \\
\hline 16
\end{tabular}

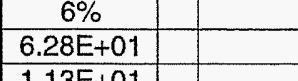

$1.13 \mathrm{E}+01$

\begin{tabular}{rr|r|r}
\hline Statistical value & $3.4 \mathrm{E}+00$ & \\
\hline Most Stringent Cleanup Limit for & $3.4 \mathrm{E}+00$ & \\
\hline \multicolumn{3}{|c|}{ Direct } \\
\hline
\end{tabular}

\begin{tabular}{l}
$.75 E+01$ \\
$.211+01$ \\
\hline $.75 E+01$
\end{tabular}

3 WAC 173-340 3-PART Test

\begin{tabular}{l|l}
14 & $95 \%$ UCL $>$ Cleanup Limit? \\
15 & $>10 \%$ above Cleanup Limit?
\end{tabular}

Any sample > 2X Cleanup Limi
17
18 EXCESS RISK EVALUATION:
WAC 173-340 Noncarcinogenic Cleanup

AC 173-340 Noncarcinogenic Cleanup

WAC 173-340 Carcinogenic Cleanup

(a)

WAC 173-340 3-Part-Test
Compliance?

YES

Because all arsenic
values are below

Nonrad noncarcinogenic

4.1E-02

the 3-part test and

24 Nonrad carcinogenic risk:

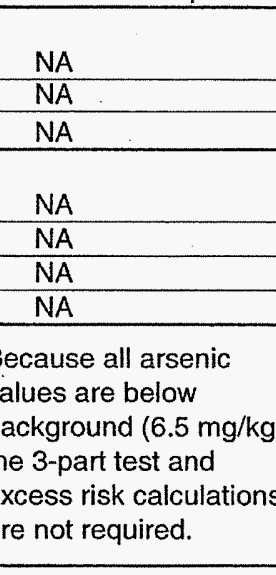

not detected in any

$132 \mathrm{mg} / \mathrm{kg}$ ), the 3-part sample, the 3-part

lest and excess risk and excess risk

required.
CALCULATION SHEET

Calc. No. 0600X-CA-V0060
Checked J.M. Capron Oence

Rev. No. $\frac{0}{\text { Date }}$

Sheet No. $\frac{11 / 01 / 06}{16 \text { of } 21}$

\begin{tabular}{|c|c|c|c|c|c|c|c|c|c|c|c|}
\hline \multirow{2}{*}{\multicolumn{2}{|c|}{$\begin{array}{l}\text { Chromium } \\
\text { w } \\
\begin{array}{c}\text { Large data set (n } \geq 10), \\
\text { Luse MTCAStat lognorma } \\
\text { distribution. }\end{array}\end{array}$}} & \multirow{2}{*}{\multicolumn{2}{|c|}{\begin{tabular}{|l} 
Lead \\
Large data set $(n \geq 10)$, \\
use MTCAStat \\
lognormal distribution.
\end{tabular}}} & \multirow{2}{*}{\multicolumn{2}{|c|}{\begin{tabular}{|l} 
Selenium \\
$>50 \%$ of data are below \\
detection. Default to \\
max value.
\end{tabular}}} & \multirow{2}{*}{\multicolumn{2}{|c|}{$\begin{array}{l}\text { Silver } \\
50 \% \text { of data are below } \\
\text { detection. Default to max } \\
\text { value. }\end{array}$}} & \multirow{2}{*}{\multicolumn{2}{|c|}{\begin{tabular}{|l} 
Tin \\
$>50 \%$ of data are below \\
detection. Default to $\max$ \\
value.
\end{tabular}}} & \multirow{2}{*}{\multicolumn{2}{|c|}{ 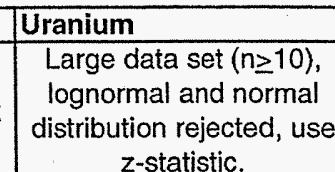 }} \\
\hline & & & & & & & & & & & \\
\hline 16 & & 16 & & 16 & & 16 & & & & 16 & \\
\hline $0 \%$ & & & & $100 \%$ & & $100 \%$ & & $100 \%$ & & $0 \%$ & \\
\hline $6.0 \mathrm{E}+00$ & & $3.8 \mathrm{E}+00$ & & $7.0 E-01$ & & $1.4 \mathrm{E}+00$ & & $1.42 E+01$ & & $\frac{2.29 E+00}{2515+0}$ & \\
\hline $7.6 E-01$ & & \begin{tabular}{|c|}
$5.6 \mathrm{E}-01$ \\
16.5
\end{tabular} & & $\begin{array}{l}1.1 \mathrm{IE}-16 \\
1.15\end{array}$ & & $\frac{2.6 \mathrm{E}-02}{1615}$ & & $7.76 \mathrm{E}-02$ & & $2.51 E+00$ & \\
\hline $\begin{array}{c}1.645 \\
6.4 \mathrm{~F}+00\end{array}$ & & \begin{tabular}{|c|}
1.645 \\
$4.0 F+00$ \\
\end{tabular} & & 1.645 & & 1.645 & & $\frac{1.645}{N A}$ & & $\frac{1.645}{0}$ & \\
\hline$\frac{6.4 \mathrm{E}+00}{7.8 \mathrm{E}+00}$ & & $\mid$\begin{tabular}{|l|}
$4.0 \mathrm{E}+00$ \\
$5.1 \mathrm{E}+00$
\end{tabular} & & $\frac{\mathrm{NA}}{1.4 \mathrm{E}+00}$ & & \begin{tabular}{c|c} 
NA & (2.9E+00 \\
\end{tabular} & & \begin{tabular}{|c|c|} 
NA & \\
$2.88 E+01$ & $U$
\end{tabular} & & $\frac{3.32+00}{1.12 E+01}$ & \\
\hline$\frac{1.0 \mathrm{~L}}{6.4 \mathrm{E}+00}$ & & \begin{tabular}{|l|}
$4.0 \mathrm{E}+00$ \\
\end{tabular} & & \begin{tabular}{c|c}
$.4 \mathrm{~L}$ \\
$1.4 \mathrm{E}+00$ & $\mathrm{U}$
\end{tabular} & & \begin{tabular}{l|l}
$2.9 \mathrm{E}+00$ & $\mathrm{U}$ \\
2
\end{tabular} & & $\mid \begin{array}{ll}2.88 E+01 & U \\
2.88 E+01 & U\end{array}$ & & $3.32 \mathrm{E}+00$ & \\
\hline 120,000 & $\begin{array}{c}\text { Direct } \\
\text { Exposure }\end{array}$ & 353 & $\begin{array}{c}\text { Direct } \\
\text { Exposure }\end{array}$ & 400 & $\begin{array}{c}\text { Direct } \\
\text { Exposure }\end{array}$ & 400 & $\begin{array}{c}\text { Direct } \\
\text { Exposure }\end{array}$ & 48,000 & $\begin{array}{l}\text { Direct } \\
\text { Exposure }\end{array}$ & 81 & $\begin{array}{c}\text { Direct } \\
\text { Exposure } \\
\end{array}$ \\
\hline NA & & $\mathrm{NA}$ & & NA & & $\mathrm{NA}$ & & $\mathrm{NA}$ & & No & \\
\hline NA & & NA & & NA & & NA & & $\mathrm{NA}$ & & NO & \\
\hline $\mathrm{NA}$ & & $\mathrm{NA}$ & & NA & & $\mathrm{NA}$ & & $\mathrm{NA}$ & & NO & \\
\hline NA & & $\mathrm{NA}$ & & NA & & $\mathrm{NA}$ & & NA & & & \\
\hline & & $\mathrm{NA}$ & & NA & & NA & & $\mathrm{INA}$ & & $4.1 \mathrm{E}-02$ & \\
\hline $\mathrm{NA}$ & & $\mathrm{NA}$ & & $N$ & & $\mathrm{NA}$ & & 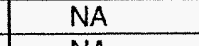 & & NA & \\
\hline NA & & NA & & $\mathrm{NA}$ & & NA & & NA & & NA & \\
\hline $\begin{array}{l}\text { Because all } \\
\text { values are be } \\
\text { background } \\
\text { mg/kg), the } 3 \\
\text { and excess } r \\
\text { calculations } \\
\text { required. }\end{array}$ & $\begin{array}{l}\text { chromium } \\
\text { elow } \\
\text { (18.5 } \\
\text { 3-part test } \\
\text { risk } \\
\text { are not }\end{array}$ & $\begin{array}{l}\text { Because all } \\
\text { are below b } \\
\text { (10.2 mg/kg } \\
\text { test and exc } \\
\text { calculations } \\
\text { required. }\end{array}$ & $\begin{array}{l}\text { lead values } \\
\text { ackground } \\
\text {, the 3-part } \\
\text { ess risk } \\
\text { are not }\end{array}$ & $\begin{array}{l}\text { Because sele } \\
\text { not detected } \\
\text { sample, the } 3 \\
\text { and excess ri } \\
\text { calculations a } \\
\text { required. }\end{array}$ & $\begin{array}{l}\text { lenium was } \\
\text { in any } \\
\text { 3-part test } \\
\text { risk } \\
\text { are not }\end{array}$ & $\begin{array}{l}\text { Because silver } \\
\text { detected in an } \\
\text { the 3-part test } \\
\text { risk calculation } \\
\text { required. }\end{array}$ & $\begin{array}{l}\text { r was not } \\
\text { y sample, } \\
\text { and excess } \\
\text { ns are not }\end{array}$ & $\begin{array}{l}\text { Because tin we } \\
\text { detected in an } \\
\text { the 3-part test } \\
\text { risk calculation } \\
\text { required. }\end{array}$ & $\begin{array}{l}\text { vas not } \\
\text { ay sample, } \\
t \text { and excess } \\
\text { ns are not }\end{array}$ & $\begin{array}{l}\text { The data se } \\
\text { part test in } \\
\text { the most st }\end{array}$ & $\begin{array}{l}\text { meets the } \\
\text { omparison to } \\
\text { ingent } R A G \text {. }\end{array}$ \\
\hline
\end{tabular}


Originator K. A. Anselm $1 C A C$

Date 11/01/06

Job No. 14655

Calc. No. 0600X-CA-V0060

Subject Cleanup Verification $95 \%$ UCL Calculations for 618-2 Shallow Zone/Deep Zone/Overburden

Checked J.M. Capron \&zec

$\frac{0}{11 / 01 / 06}$ and 618-2, 618-3, 618-8 Staging Pile and Decon Pad Footprint

Ecology Software (MTCAStat) Results for 618-2, 618-3, and 618-8 Staging Pile/Decon Pad Footprint

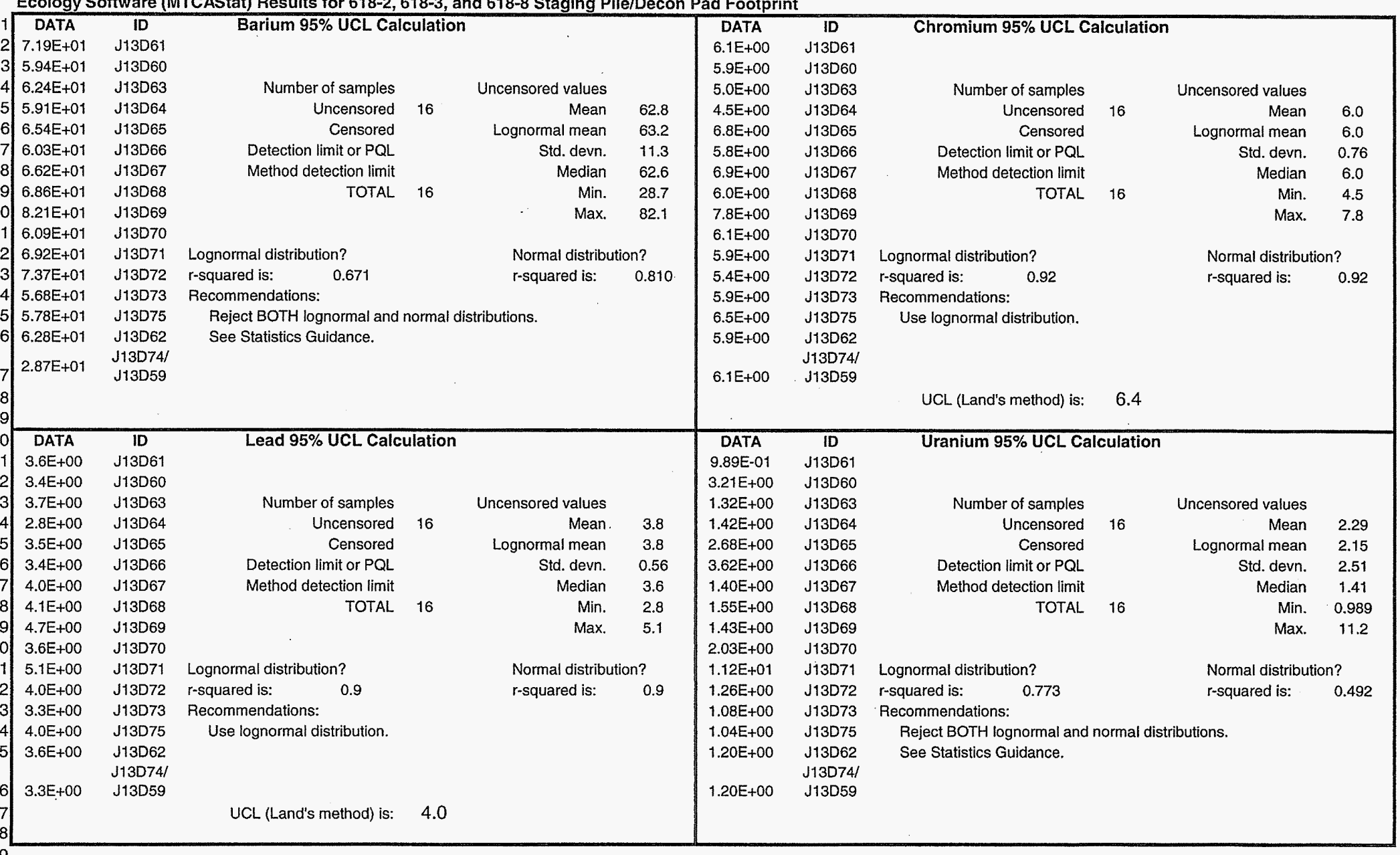


CVP-2006-00010

Rev. 0

D-21 


\section{Split-Duplicate Analysis}

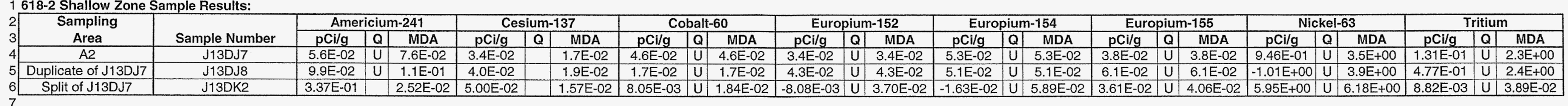

8 Sample Analysis:

\begin{tabular}{|c|c|c|c|c|c|c|c|c|c|}
\hline & $\mathrm{DL}$ & 1 & 0.1 & 0.05 & 0.1 & 0.1 & 0.1 & 30 & 400 \\
\hline \multirow[b]{2}{*}{ Duplicate Analysis } & Both $>\mathrm{PQL}$ or MDA? & No-Stop (acceptable) & Yes (continue) & No-Stop (acceptable) & No-Stop (acceptable) & No-Stop (acceptable) & No-Stop (acceptable) & No-Stop (acceptable) & No-Stop (acceptable) \\
\hline & Both > 5xTDL? & & No-Stop (acceptable) & & & & & & \\
\hline \multirow[b]{2}{*}{ Split Analysis } & Both $>$ PQL? & No-Stop (acceptable) & Yes (continue) & No-Stop (acceptable) & No-Stop (acceptable) & No-Stop (acceptable) & No-Stop (acceptable) & No-Stop (acceptable) & No-Stop (acceptable) \\
\hline & $\begin{array}{c}\text { Both > 5xTDL? } \\
\text { RPD }\end{array}$ & & No-Stop (acceptable) & & & & & & \\
\hline
\end{tabular}

18 618-2 Shallow Zone Sample Results (cont.)

\begin{tabular}{|c|c|c|c|c|c|c|c|c|c|c|c|c|c|c|c|c|c|c|c|}
\hline \multirow{3}{*}{\multicolumn{2}{|c|}{$\begin{array}{c}\text { Sampling } \\
\text { Area } \\
\text { A2 }\end{array}$}} & \multirow[b]{2}{*}{ Sample Number } & \multicolumn{3}{|c|}{ Plutonium-238 } & \multicolumn{2}{|c|}{ Plutonium-239/240 } & \multicolumn{2}{|c|}{ Plutonium-241 } & \multicolumn{3}{|c|}{ Strontium-90 } & \multicolumn{2}{|c|}{ Uranium-233/234 } & \multicolumn{3}{|c|}{ Uranium-235 } & \multicolumn{2}{|c|}{ Uranium-238 } \\
\hline & & & pcilg & Q & MDA & $\mathrm{pCi} / \mathrm{g}$ & $\overline{M D A}$ & $\mathrm{pCi} / \mathrm{g}$ & \begin{tabular}{|l|l}
$Q$ & MDA \\
\end{tabular} & $\mathrm{pCi} / \mathrm{g}$ & Q & MDA & $\mathrm{pCi} / \mathrm{g}$ & MDA & $\mathrm{pCi} / \mathrm{g}$ & Q & MDA & \begin{tabular}{l|l}
$\mathrm{pCi} / \mathrm{g}$ & $\mathrm{c}$ \\
\end{tabular} & $\overline{M D A}$ \\
\hline & A2 & J13DJ7 & $5.9 \mathrm{E}-02$ & $\underline{u}$ & 4.5E-01 & \begin{tabular}{|l|}
$8.24 \mathrm{E}-01$ \\
\end{tabular} & $4.5 \mathrm{E}-01$ & $1.42 \mathrm{E}+01$ & $2.0 \mathrm{E}+01$ & $-1.4 \mathrm{E}-02$ & $u$ & $2.2 \mathrm{E}-01$ & $1.71 \mathrm{E}+00$ & $1.7 \mathrm{E}-01$ & $2.14 \mathrm{E}-01$ & & $2.1 \mathrm{E}-01$ & $1.77 \mathrm{E}+00$ & $1.7 \mathrm{E}-01$ \\
\hline & $\frac{\text { Duplicate of } \mathrm{J13DJ7}}{\text { Split of } \mathrm{J} 13 \mathrm{DJ} 7}$ & $\frac{\text { J13DJ8 }}{\text { J13DK2 }}$ & $\frac{6.8 \mathrm{E}-02}{1.57 \mathrm{~F}-2}$ & $\frac{U}{u}$ & $\frac{2.6 \mathrm{E}-01}{202 \mathrm{E}-02}$ & \begin{tabular}{|l|l|}
$7.18 \mathrm{E}-01$ \\
$173 \mathrm{~F}-00$ \\
\end{tabular} & $\frac{2.6 E-01}{202-02}$ & $9.65 E+00$ & \begin{tabular}{|l|l|} 
& $1.8 E+01$ \\
& $0.70 E-01$
\end{tabular} & $\frac{-2.4 \mathrm{E}-02}{0.02}$ & $u$ & $\frac{2.5 \mathrm{E}-01}{126 \mathrm{E}-1}$ & \begin{tabular}{|l|l|}
$1.83 E+00$ \\
$502 \mathrm{E}+00$
\end{tabular} & $\frac{2.3 E-01}{384 E^{-29}}$ & $\frac{1.11 \mathrm{E}-01}{1.15 \mathrm{~F}-01}$ & $u$ & $\frac{2.8 \mathrm{E}-01}{3.23 \mathrm{E}-02}$ & \begin{tabular}{|l|}
$1.89 \mathrm{E}+00$ \\
$408 \mathrm{~F}+00$ \\
\end{tabular} & $\frac{2.3 \mathrm{E}-01}{2.61 \mathrm{E}-02}$ \\
\hline
\end{tabular}

23 Split of J13DJ7

25 Sample Analysis:

\begin{tabular}{|c|c|c|c|c|c|c|c|c|}
\hline \multicolumn{2}{|c|}{ TDL } & 1 & 1 & 15 & 1 & 1 & 1 & 1 \\
\hline \multirow[b]{2}{*}{ Duplicate Analysis } & Both $>$ PQL or MDA? & No-Stop (acceptable) & Yes (continu & No-Stop (acceptable) & No-Stop (acceptable) & Yes (continue) & No-Stop (acceptable) & Yes (continue) \\
\hline & Both > 5xTDL? & & No-Stop (acceptable) & & & No-Stop (acceptable) & & No-Stop (acceptable) \\
\hline \multirow[b]{2}{*}{ Split Analysis } & & No-S & & be) & No-St & & & \\
\hline & $\begin{array}{l}\text { Both > 5xTDL? } \\
\text { RPD }\end{array}$ & & No-Stop (acceptable) & & & No-Stop (acceptable) & No-Stop (acceptable) & No-Stop (acceptable) \\
\hline
\end{tabular}

33

35 618-2 Shallow Zone Sample Results (cont.)

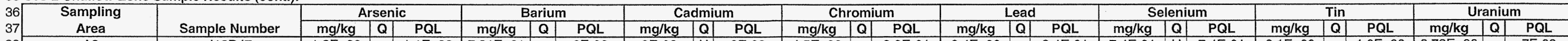

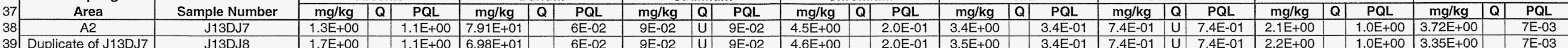

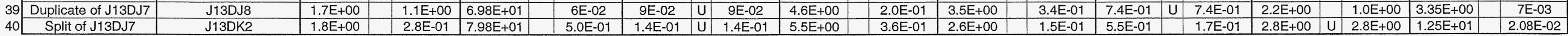
40 Split of J13DJ7

42 Sample Analysis:

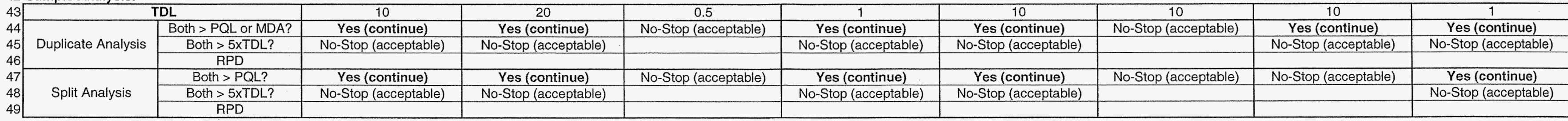




\section{Split-Duplicate Analysis}

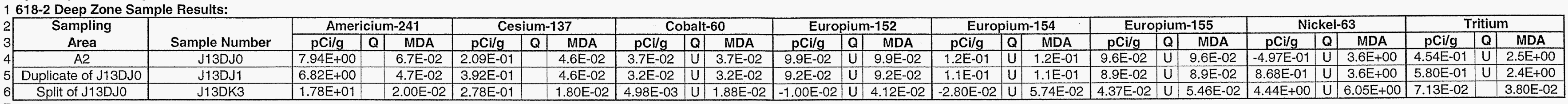

8 Sample Analysis:

\begin{tabular}{|c|c|c|c|c|c|c|c|c|c|}
\hline+1 & $\mathrm{DL}$ & 1 & 0.1 & 0.05 & 0.1 & 0.1 & 0.1 & 30 & 400 \\
\hline \multirow[b]{2}{*}{ Duplicate Analysis } & Both > PQL or MDA? & Yes (continue) & Yes (continue) & No-Stop (acceptable) & No-Stop (acceptable) & No-Stop (acceptable) & No-Stop (acceptable) & No-Stop (acceptable) & No-Stop (acceptable) \\
\hline & Both > 5xTDL? & Yes (calc RPD) & No-Stop (acceptable) & & & & & & \\
\hline \multirow{3}{*}{ Split Analysis } & $\frac{\text { RPD }}{\text { Both > PQL? }}$ & $\frac{15 \%}{\text { Yes (continue) }}$ & Yes (continue) & No-Stop (acceptable) & No-Stop (acceptable) & No-Stop (acceptable) & No-Stop (acceptable) & No-Stop (acceptable) & No-Stop (acceptable) \\
\hline & Both > 5xTDL? & Yes (calc RPD) & No-Stop (acceptable) & & & & & & \\
\hline & GPD & $77 \%$ & & & & & & & \\
\hline
\end{tabular}

18 618-2 Deep Zone Sample Results (cont.):

\begin{tabular}{|c|c|c|c|c|c|c|c|c|c|c|c|c|c|c|c|c|}
\hline \multirow{2}{*}{ 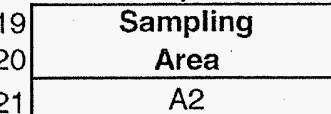 } & \multirow[b]{2}{*}{ Sample Number } & \multicolumn{3}{|c|}{ Plutonium-238 } & \multicolumn{2}{|c|}{ Plutonium-239/240 } & \multicolumn{2}{|c|}{ Plutonium-241 } & \multicolumn{2}{|c|}{ Strontium-90 } & \multicolumn{2}{|c|}{ Uranium-233/234 } & \multicolumn{2}{|c|}{ Uranium-235 } & \multicolumn{2}{|c|}{ Uranium-238 } \\
\hline & & $p \mathrm{Ci} / \mathrm{g}$ & $\begin{array}{lll}a & & \\
\end{array}$ & MDA & $p$ cilg & MDA & \begin{tabular}{l|l}
$\mathrm{pC} \mathrm{Ci} / \mathrm{g}$ & $\mathrm{c}$ \\
\end{tabular} & MDA & pCi/g & MDA & $p \mathrm{Ci} / \mathrm{g}$ & \begin{tabular}{|l|l|} 
Q & MDA \\
\end{tabular} & $\mathrm{pCi} / \mathrm{g}$ & \begin{tabular}{l|l} 
Q & MDA \\
\end{tabular} & $\mathrm{pCi} / \mathrm{g}$ & \begin{tabular}{l|l} 
Q & MDA \\
\end{tabular} \\
\hline $\mathrm{A} 2$ & J13DJO & $4.17 \mathrm{E}-01$ & $\mathrm{u}$ & $5.3 \mathrm{E}-01$ & $5.16 \mathrm{E}+01$ & $5.3 \mathrm{E}-01$ & $2.97 \mathrm{E}+01$ & $2.9 \mathrm{E}+01$ & $1.16 \mathrm{E}+01$ & 2.1E-01 & $1.46 \mathrm{E}+01$ & $2.9 \mathrm{E}-01$ & 7.76E-01 & $2.8 \mathrm{E}-01$ & $1.48 \mathrm{E}+01$ & $2.9 \mathrm{E}-01$ \\
\hline Duplicate of J13DJ0 & J13DJ1 & 4.15E-01 & $\mathrm{u}$ & $4.5 \mathrm{E}-01$ & $4.55 \mathrm{E}+01$ & 4.5E-01 & $9.69 E+00 \mathrm{~L}$ & \begin{tabular}{l|l} 
U & $2.2 \mathrm{E}+01$ \\
\end{tabular} & $9.39 E+00$ & $2.0 \mathrm{E}-01$ & $1.28 \mathrm{E}+01$ & $3.0 \mathrm{E}-01$ & 7.61E-01 & $2.5 \mathrm{E}-01$ & $25 \mathrm{E}+$ & $6 \mathrm{E}-0$ \\
\hline Split of J13DJ0 & J13DK3 & $1.32 \mathrm{E}+00$ & & $2.19 \mathrm{E}-02$ & $9.23 \mathrm{E}+01$ & $2.19 \mathrm{E}-02$ & $2.65 \mathrm{E}+01$ & $8.06 \mathrm{E}-01$ & $1.46 \mathrm{E}+01$ & $1.21 \mathrm{E}-01$ & $2.94 \mathrm{E}+01$ & $3.44 \mathrm{E}-02$ & $0 E+00$ & $.89 \mathrm{E}-02$ & $95 \mathrm{E}+0$ & $09 E-C$ \\
\hline
\end{tabular}

25 Sample Analysis:

\begin{tabular}{|c|c|c|c|c|c|c|c|c|}
\hline \multirow{4}{*}{\begin{tabular}{|l} 
Duplicate Analysis \\
\end{tabular}} & & 1 & 1 & 15 & 1 & & 1 & \multirow{3}{*}{$\begin{array}{l}\text { Yes (continue) } \\
\text { Yes (calc RPD) }\end{array}$} \\
\hline & \begin{tabular}{|l|l|l|l|} 
TDL \\
Both $>$ PQL or MDA?
\end{tabular} & No-Stop (acceptable) & Yes (continue) & No-Stop (acceptable) & Yes (continue) & Yes (continue) & Yes (continue) & \\
\hline & \multirow[t]{3}{*}{ Both > bx I DL? } & & Yes (calc RPD) & & Yes (calc RPD) & Yes (calc RPD) & No-Stop (acceptable) & \\
\hline & & & $13 \%$ & & $21 \%$ & $13 \%$ & & \multirow{3}{*}{$\begin{array}{c}17 \% \\
\text { Yes (continue) } \\
\text { Yes (calc RPD) }\end{array}$} \\
\hline \multirow{2}{*}{ Split Analysis } & & No-Stop (acceptable) & Yes (continue) & Yes (continue) & Yes (continue) & Yes (continue) & Yes (col & \\
\hline & $\begin{array}{c}\text { Both > 5xTDL? } \\
\text { PDD }\end{array}$ & & $\frac{Y e s(\text { calc RPD) }}{570}$ & No-Stop (acceptable) & $\frac{\text { Yes (calc RPD) }}{23 \%}$ & $\begin{array}{l}\text { Yes (calc RPD) } \\
67 \%\end{array}$ & No-Stop (acceptable) & \\
\hline
\end{tabular}

33

35 618-2 Deep Zone Sample Results (cont.):

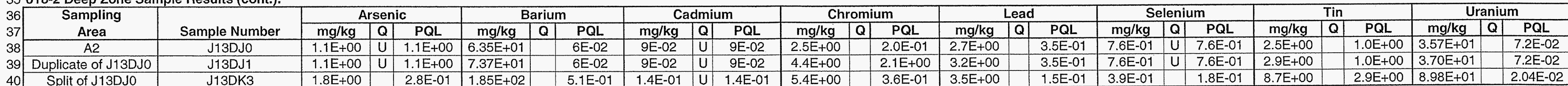

41 Spll orvisibJo

42 Sample Analysis:

\begin{tabular}{|c|c|c|c|c|c|c|c|c|c|}
\hline & $\mathrm{LL}$ & 10 & 20 & 0.5 & 1 & 10 & 10 & 10 & 1 \\
\hline \multirow[b]{2}{*}{ Duplicate Analysis } & Both > PQL or MDA? & No-Stop (acceptable) & Yes (continue) & No-Stop (acceptable) & Yes (continue) & Yes (continue) & No-Stop (acceptable) & Yes (continue) & Yes (continue) \\
\hline & $\begin{array}{l}\text { Both > 5xTDL? } \\
\text { RPD }\end{array}$ & & No-Stop (acceptable) & & No-Stop (acceptable) & No-Stop (acceptable) & & No-Stop (acceptable) & $\frac{\text { Yes (calc RPD) }}{4 \%}$ \\
\hline \multirow{2}{*}{ Split Analysis } & Both > PQL? & No-Stop (acceptable) & Yes (continu & No-Stop (acceptable) & 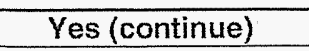 & Yes (continue) & No-Stop (acceptable) & Yes (continue & Yes (continue) \\
\hline & Both > 5xTDL? & & No-Stop (acceptable) & & No-Stop (acceptable) & No-Stop (acceptable) & & No-Stop (acceptable) & Yes (calc RPD) \\
\hline
\end{tabular}

59 Note: The significance of the RPD values, including values greater than $30 \%$, is addressed in the data quality assessment section of the CVP. 
Originator K. A. Anselm $\mathrm{Cal}$

Date $\frac{11 / 20 / 06}{1465}$

6

Subject Cleanup Verification 95\% UCL Calculations for 618-2 Shallow Zone/Deep Zone/Overburden and 618-2, 618-3, 618-8 Staging Pile and Decon Pad Footprin

Split-Duplicate Analysis

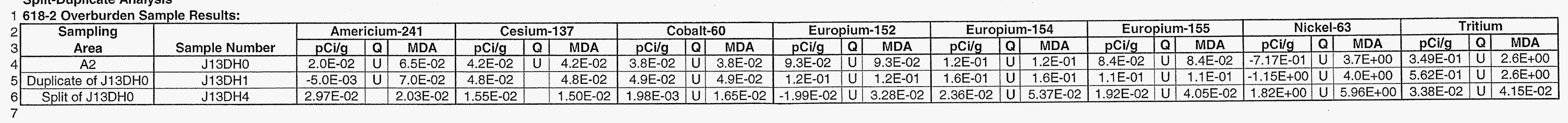

\begin{tabular}{|c|c|c|c|c|c|c|c|c|c|}
\hline & $\mathrm{DL}$ & 1 & 0.1 & 0.05 & 0.1 & 0.1 & 0.1 & 30 & 400 \\
\hline \multirow[b]{2}{*}{ Duplicate Analysis } & Both $>$ PQL or MDA? & No-Stop (acceptable) & No-Stop (acceptable) & No-Stop (acceptable) & No-Stop (acceptable) & No-Stop (acceptable) & No-Stop (acceptable) & No-Stop (acceptable) & No-Stop (acceptable) \\
\hline & $\begin{array}{l}\text { Both > 5xTDL? } \\
\text { BPD }\end{array}$ & & & & & & & & \\
\hline \multirow[b]{2}{*}{ Split Analysis } & Both > PQL? & No-Stop (acceptable) & No-Stop (acceptable) & No-Stop (acceptable) & No-Stop (acceptable) & No-Stop (acceptable) & No-Stop (acceptable) & No-Stop (acceptable) & No-Stop (acceptable) \\
\hline & $\begin{array}{c}\text { Both > 5xTDL? } \\
\text { RPD }\end{array}$ & & & & & & & & \\
\hline
\end{tabular}

15
16

18 618-2 Overburden Sample Results (cont.):

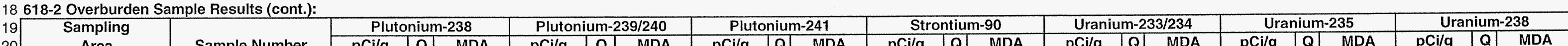

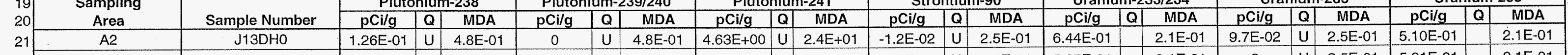

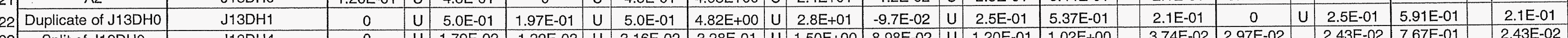

23 Split of J13DHO

25 Sample Analysis:

\begin{tabular}{|c|c|c|c|c|c|c|c|c|}
\hline (2) & TDL & 1 & 1 & 15 & 1 & 1 & 1 & 1 \\
\hline \multirow{3}{*}{ Duplicate Analysis } & Both $>$ PQL or MDA? & No-Stop (acceptable) & No-Stop (acceptable) & No-Stop (acceptable) & No-Stop (acceptable) & Yes (continue) & No-Stop (acceptable) & Yes (continue) \\
\hline & Both > 5xTDL? & & & & & No-Stop (acceptable) & & No-Stop (acceptable) \\
\hline & $\frac{M r}{B o t h}>$ & No-Stop (acceptable) & No-Stop (acceptable) & No-Stop (acceptable) & No-Stop (acceptable) & Yes (conti & No-Stop (acceptable) & Yes (continue) \\
\hline Split Analysis & Both > & & & & & No-Stop (acc & & No-Stop (acceptable) \\
\hline
\end{tabular}

32
33
34

35 618-2 Overburden Sample Results (cont.):

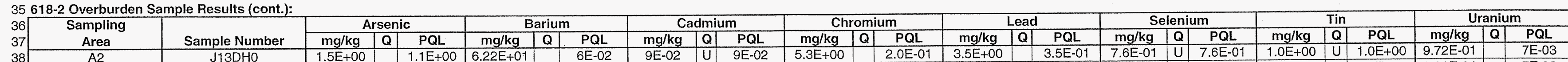

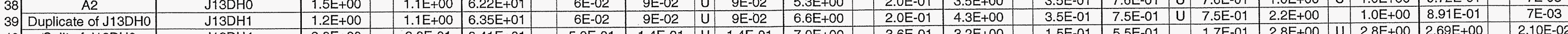

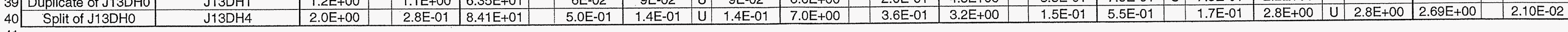

42 Sample Analysis:

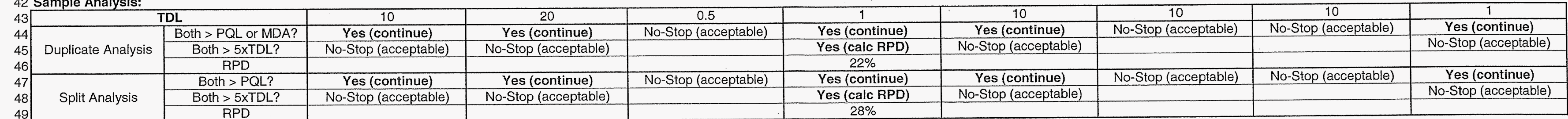

50 Note: The significance of the RPD values, including values greater than $30 \%$, is addressed in the data quality assessment section of the CVP. 
Calc. No. $0600 \mathrm{X}$-CA-V0060

\section{Split-Duplicate Analysis}

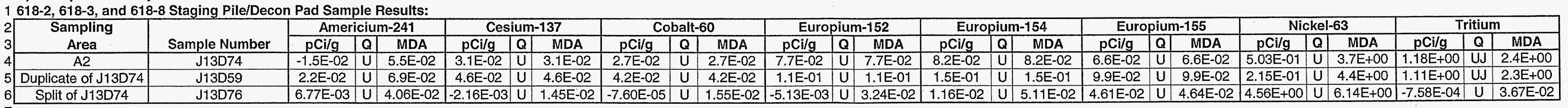

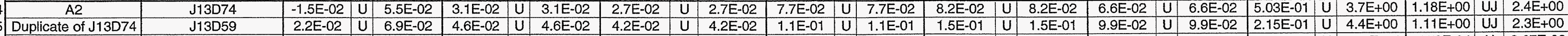

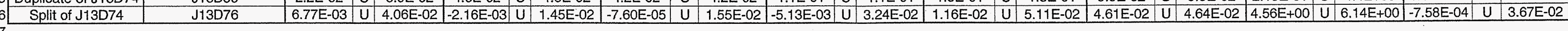

\begin{tabular}{|c|c|c|c|c|c|c|c|c|c|}
\hline \multicolumn{2}{|l|}{ Sample } & 1 & 0.1 & 0.05 & 0.1 & 0.1 & 0.1 & 30 & 400 \\
\hline \multirow[b]{2}{*}{ Duplicate Analysis } & Both $>\mathrm{PQL}$ or MDA? & No-Stop (acceptable) & No-Stop (acceptable) & No-Stop (acceptable) & No-Stop (acceptable) & No-Stop (acceptable) & No-Stop (acceptable) & No-Stop (acceptable) & No-Stop (acceptable) \\
\hline & $\begin{array}{l}\text { Both > 5xTDL? } \\
\text { BPD }\end{array}$ & & & & & & & & \\
\hline \multirow{2}{*}{ Split Analysis } & $\begin{array}{l}\text { Both > PQL? } \\
\text { Both > 5xTDL? }\end{array}$ & No-Stop (acceptable) & No-Stop (acceptable) & No-Stop (acceptable) & No-Stop (acceptable) & No-Stop (acceptable) & No-Stop (acceptable) & No-Stop (acceptable) & No-Stop (acceptable) \\
\hline & RPD & & & & & & & & \\
\hline
\end{tabular}
16

18 618-2, 618-3, and 618-8 Staging Pile/Decon Pad Sample Results (cont.):

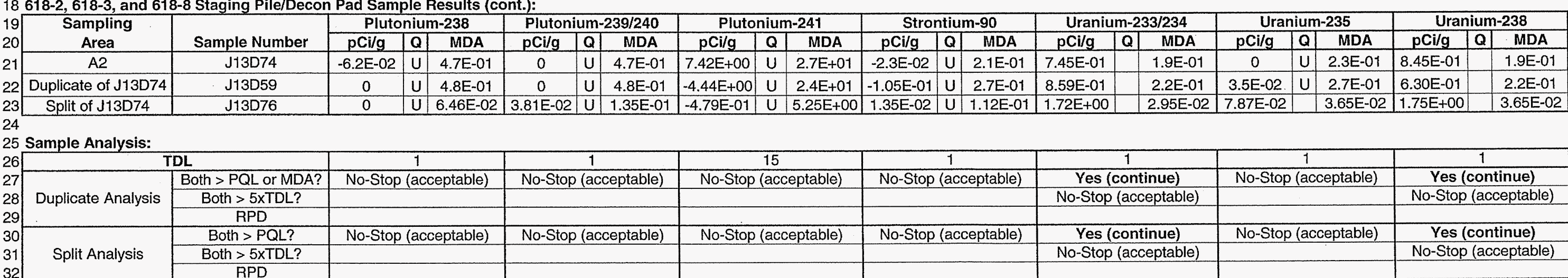

33

34

35 618-2, 618-3, and 618-8 Staging Pile/Decon Pad Sample Results (cont.):

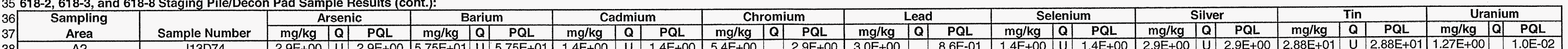

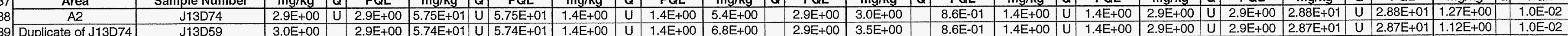

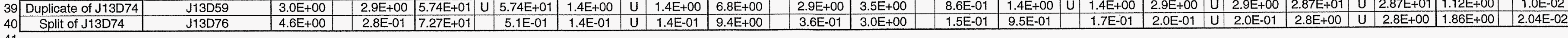

42 Sample Analysis:

\begin{tabular}{|c|c|c|c|c|c|c|c|c|c|c|}
\hline & $\mathrm{DL}$ & 10 & 20 & 0.5 & 1 & 10 & 10 & 0.2 & 10 & 1 \\
\hline \multirow{2}{*}{$\begin{array}{l}45 \\
45\end{array}$} & Both $>\mathrm{PQL}$ or MDA? & No-Stop (acceptable) & No-Stop (acceptable) & No-Stop (acceptable) & Yes (continue) & Yes (continue) & No-Stop (acceptable) & No-Stop (acceptable) & No-Stop (acceptable) & Yes (continue) \\
\hline & \begin{tabular}{|l} 
Both > 5xTDL? \\
PDD
\end{tabular} & & & & Yes (calc RPD) & No-Stop (acceptable) & & & & No-Stop (acceptable) \\
\hline \multirow[b]{2}{*}{ Split $t$} & Both $>$ & No-Stop (acceptable) & No-Stop (acceptable) & No-Stop (acceptable) & Yes (continue) & Yes (continue) & No-Stop (acceptable) & No-Stop (acceptable) & No-Stop (acceptable) & Yes (continue) \\
\hline & Both > 5xTDL? & & & & Yes (calc RPD) & No-Stop (acceptable) & & & & No-Stop (acceptable) \\
\hline
\end{tabular}

50 Note: The significance of the RPD values, including values greater than $30 \%$, is addressed in the data quality assessment section of the CVP. 


\section{CALCULATION COVER SHEET}

Project Title:

Area

Discipline

Subject

Computer Program
618-2 Burial Ground Sample Design

Job No.

14655
Environmental Engineering $\quad$ *Calc. No. 0300X-CA-V0077

618-2 Shallow, Deep Zone, Overburden/Stockpile Area Sampling Plan

Excel Program No. Excel 2003

The attached calculations have been generated to document compliance with established cleanup levels. These

calculations should be used in conjuction with other relevent documents in the administrative record.

\section{Committed Calculation $\square \quad$ Preliminary $\square$. Superseded $\square \quad$ Voided $\square$}

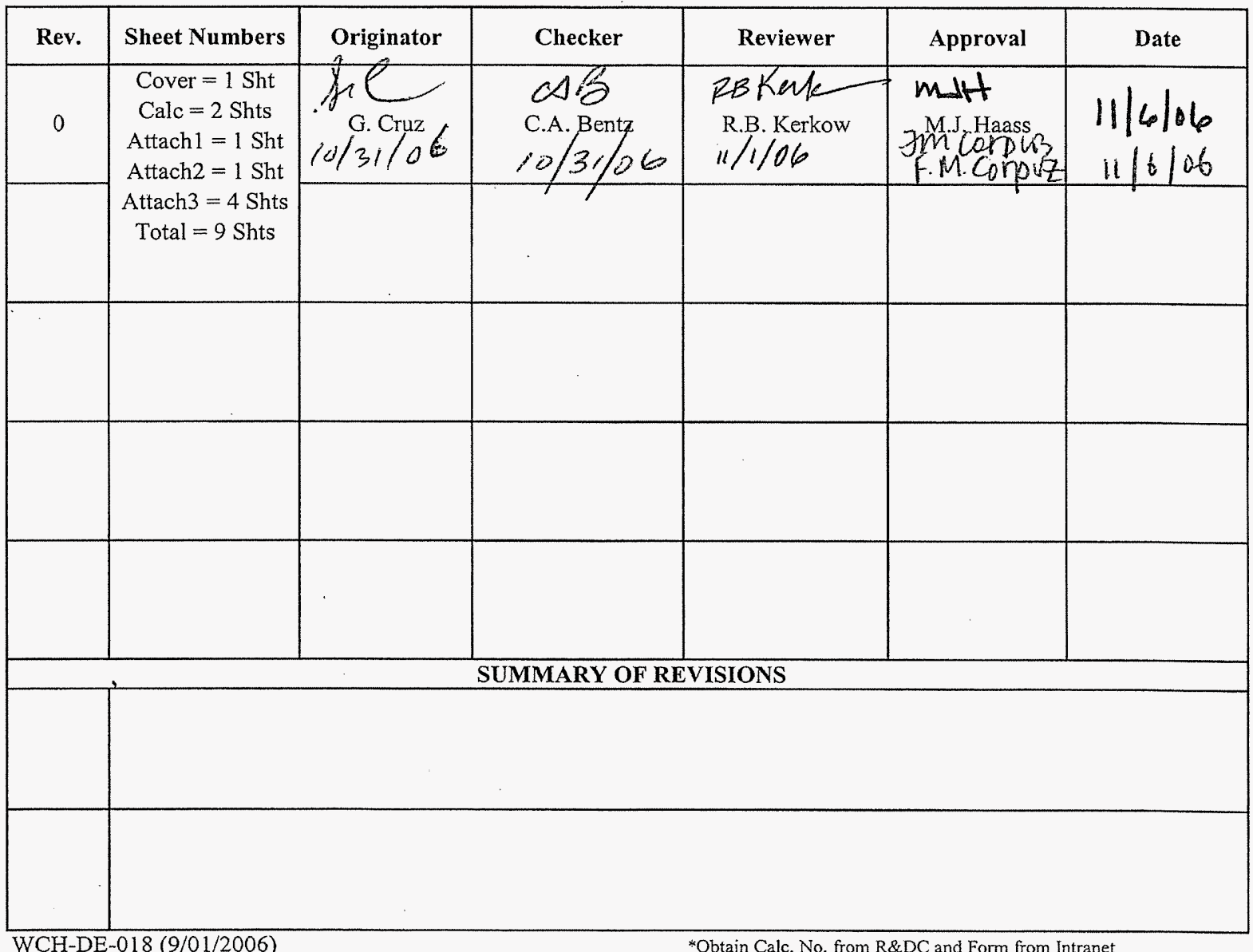




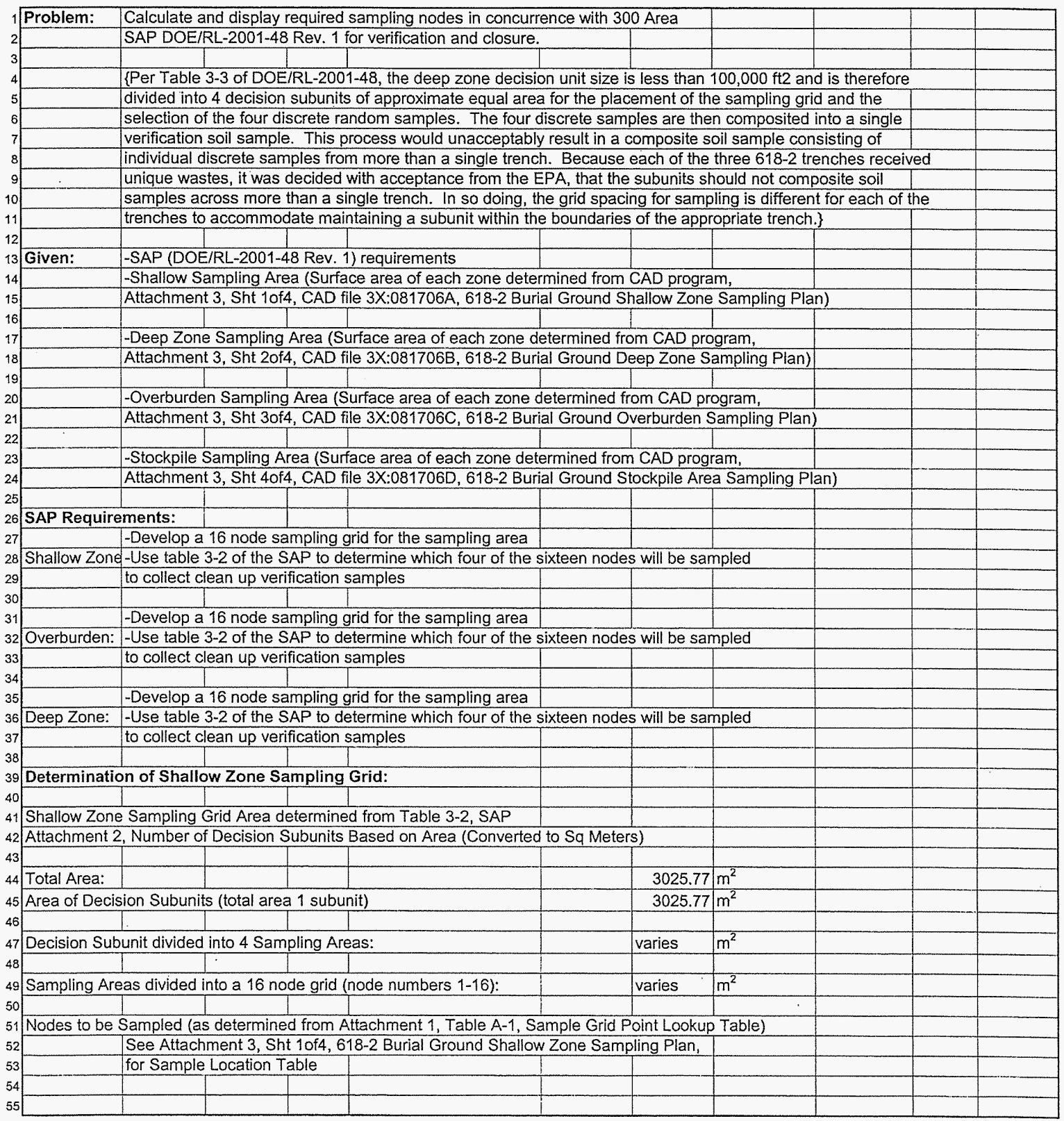




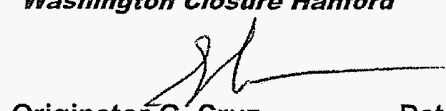

Originator $\mathrm{G}$. Cruz

Date $10 / 31 / 2006$

Project 618-2 Burial Ground Sample Design

Subject 618-2 Shallow, Deep Zone, Overburden/Stockpile Area Sampling Plan
Calc. No. 0300 X-CA-V0077 Rev. No. 0 Job No. 14655 checked aS Date $10 / 31 / 06$ Sheet No. 2 of 2

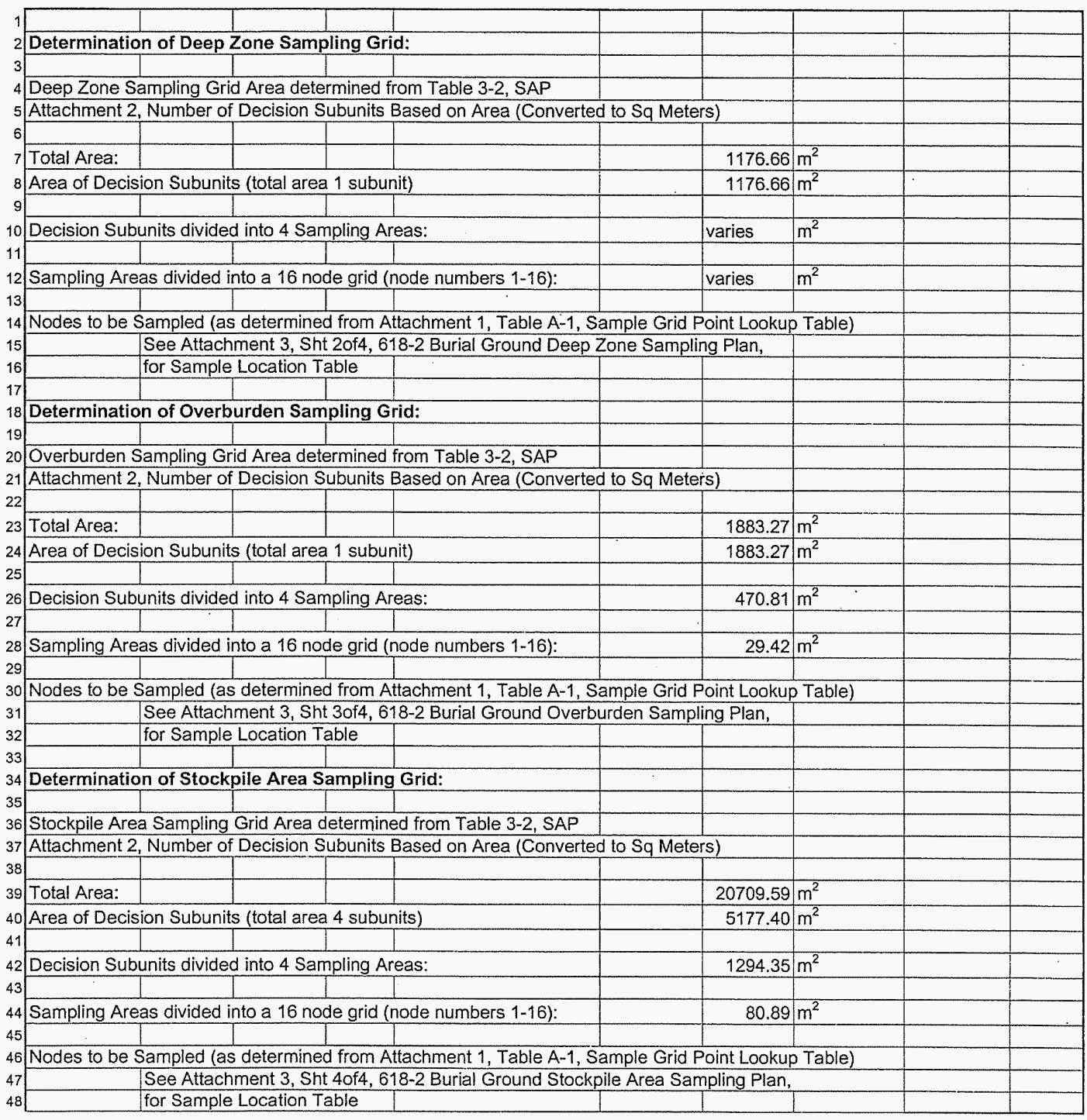


Originator $\mathbf{G}$. Cruz

Date $10 / 31 / 2006$

Calc. No. 0300X-CA-V0077 Rev. No. 0

Project 618-2 Burial Ground Sample Design Job No. 14655 Checked QSB

Subject

618-2 Shallow, Deep Zone, Overburden/Stockpile Area Sampling Plan

Sheet No. 1 of 1

\begin{tabular}{|c|c|c|c|c|c|c|}
\hline \multicolumn{7}{|c|}{${ }_{4}$ Number of Decision Subunits Based on Area. } \\
\hline $\begin{array}{l}7 \\
8\end{array}$ & \multicolumn{6}{|c|}{ Site Verification Sampling Frequencies Based on Area. } \\
\hline 10 & Decision Unit ${ }^{2}$ & Waste Site Size $^{\text {b }}$ & $\begin{array}{l}\text { Decision } \\
\text { Subunits }\end{array}$ & Blocks $^{\mathrm{c}}$ & $\begin{array}{l}\text { Discrete } \\
\text { Samples }\end{array}$ & $\begin{array}{c}\text { Composite } \\
\text { Samples }\end{array}$ \\
\hline 12 & \multirow{3}{*}{$\begin{array}{l}\text { Shallow zone- } \\
0 \text { to } 15 \mathrm{ft}\end{array}$} & Small: $\leq 100,000 \mathrm{ft}^{2}$ & 1 & 4 & 16 & 4 \\
\hline 12 & & Medium: $>100,000 \mathrm{ft}^{2} \leq 400,000 \mathrm{ft}^{2}$ & 4 & 16 & 64 & 16 \\
\hline 14 & & Large: $>400,000 \mathrm{ft}^{2}$ & 8 & 32 & 128 & 32 \\
\hline 14 & \multirow{3}{*}{$\begin{array}{l}\text { Deep Zone - } \\
>15 \mathrm{ft}\end{array}$} & Small: $\leq 100,000 \mathrm{ft}^{2}$ & 1 & 4 & 16 & 4 \\
\hline & & Medium: $>100,000 \mathrm{ft}^{2} \leq 400,000 \mathrm{ft}^{2}$ & 4 & 16 & 64 & 16 \\
\hline & & Large: $>400,000 \mathrm{ft}^{2}$ & 8 & 32 & 128 & 32 \\
\hline & \multirow{3}{*}{$\begin{array}{l}\text { Overburen/layback } \\
\text { stockpiles }\end{array}$} & Small: $\leq 100,000 \mathrm{ft}^{2}$ & 1 & 4 & 16 & 4 \\
\hline & & Medium: $>100,000 \mathrm{ft}^{2} \leq 400,000 \mathrm{ft}^{2}$ & 4 & 16 & 64 & 16 \\
\hline & & Large: $>400,000 \mathrm{ft}^{2}$ & 8 & 32 & 128 & 32 \\
\hline & \multirow{3}{*}{$\begin{array}{l}\text { Staging pile areas } \\
\text { (residual soil) }\end{array}$} & Small: $\leq 100,000 \mathrm{ft}^{2}$ & 1 & 4 & 16 & 4 \\
\hline & & Medium: $>100,000 \mathrm{ft}^{2} \leq 400,000 \mathrm{ft}^{2}$ & 4 & 16 & 64 & 16 \\
\hline & & Large: $>400,000 \mathrm{ft}^{2}$ & 8 & 32 & 128 & 32 \\
\hline
\end{tabular}

"The shallow zone, decp zone, overburden stockpile, and staging pile areas each represent single decision units. The total number of decision - units will vary because individual waste sites may not have a deep zone, overburden stockpile, and/or staging pile areas.

- Area of exposed surface after excavation or area of stockpile base (as applicable)
- Decision subunits are divided into four blocks to ensure that random sampling locations are not bunched together in one area 
CVP-2006-00010

Rev. 0

D-31 


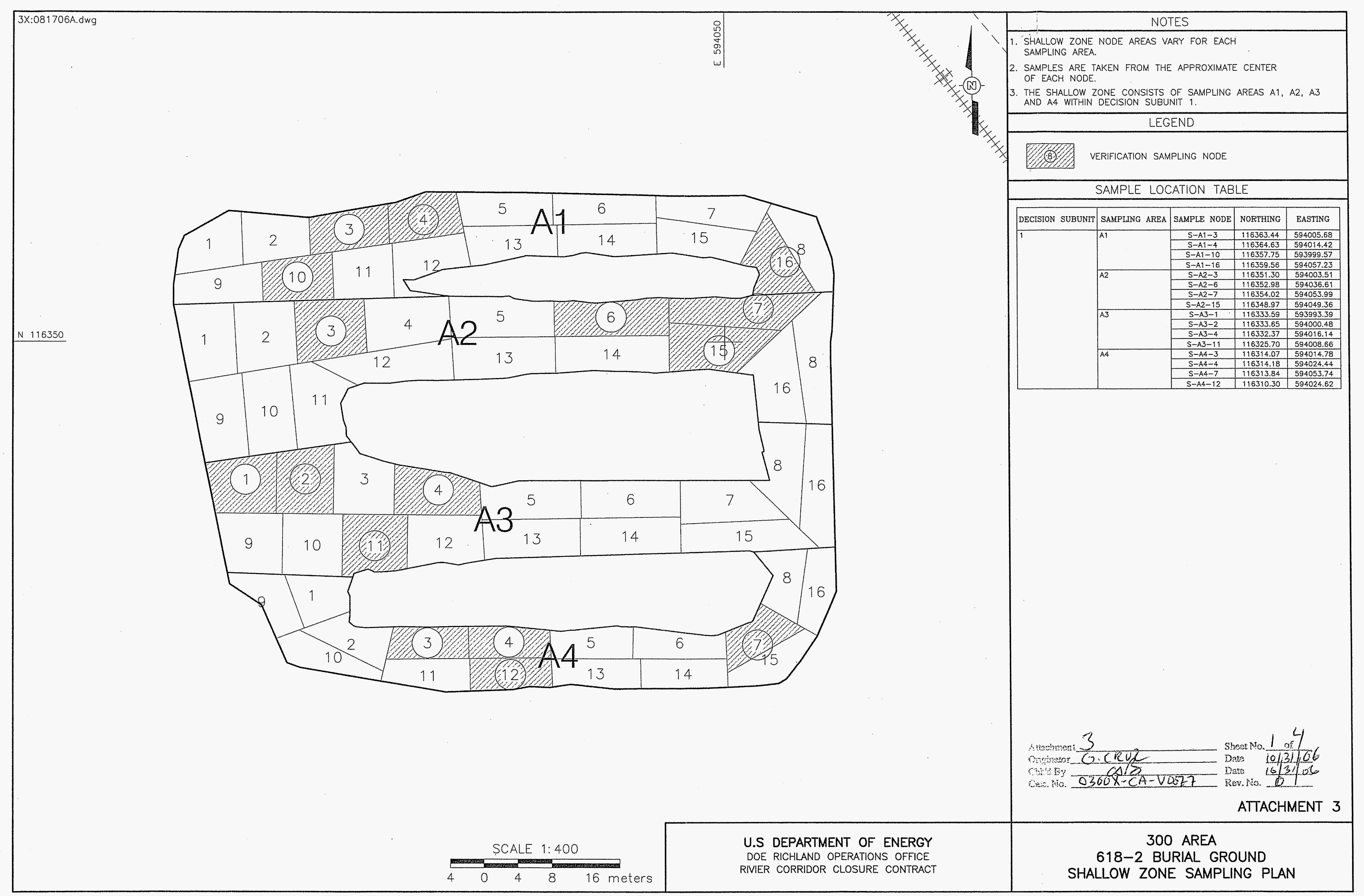




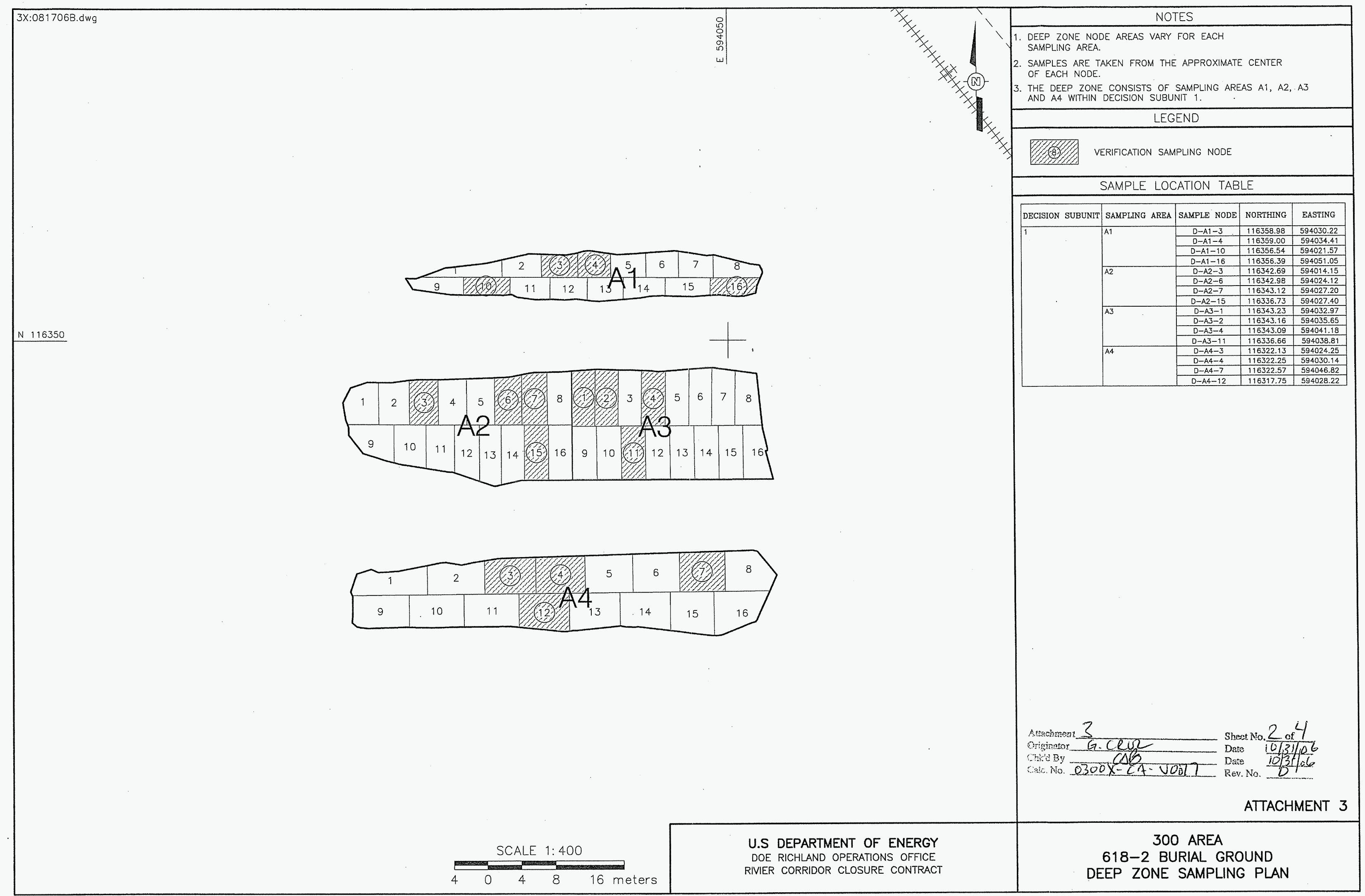




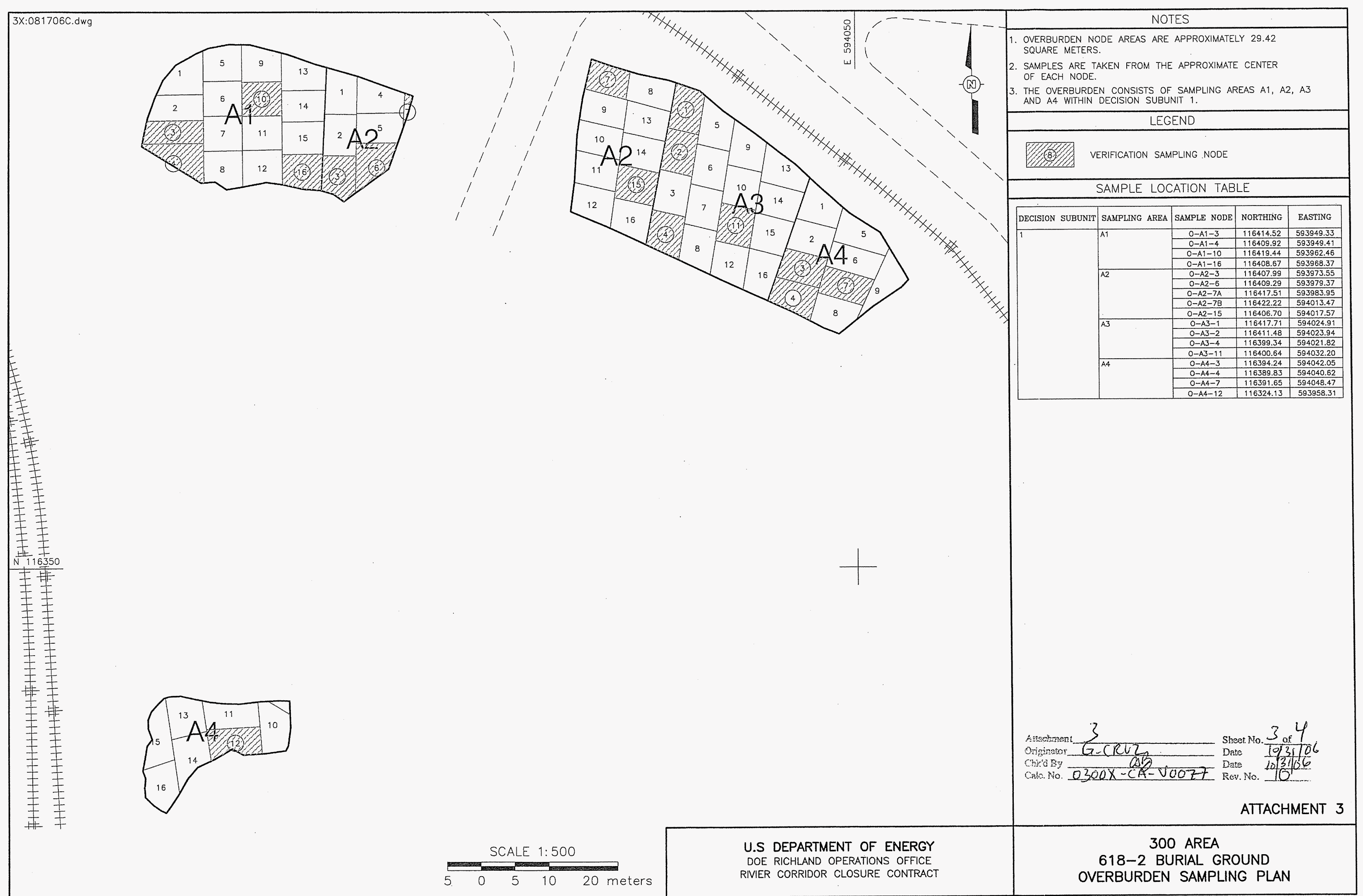




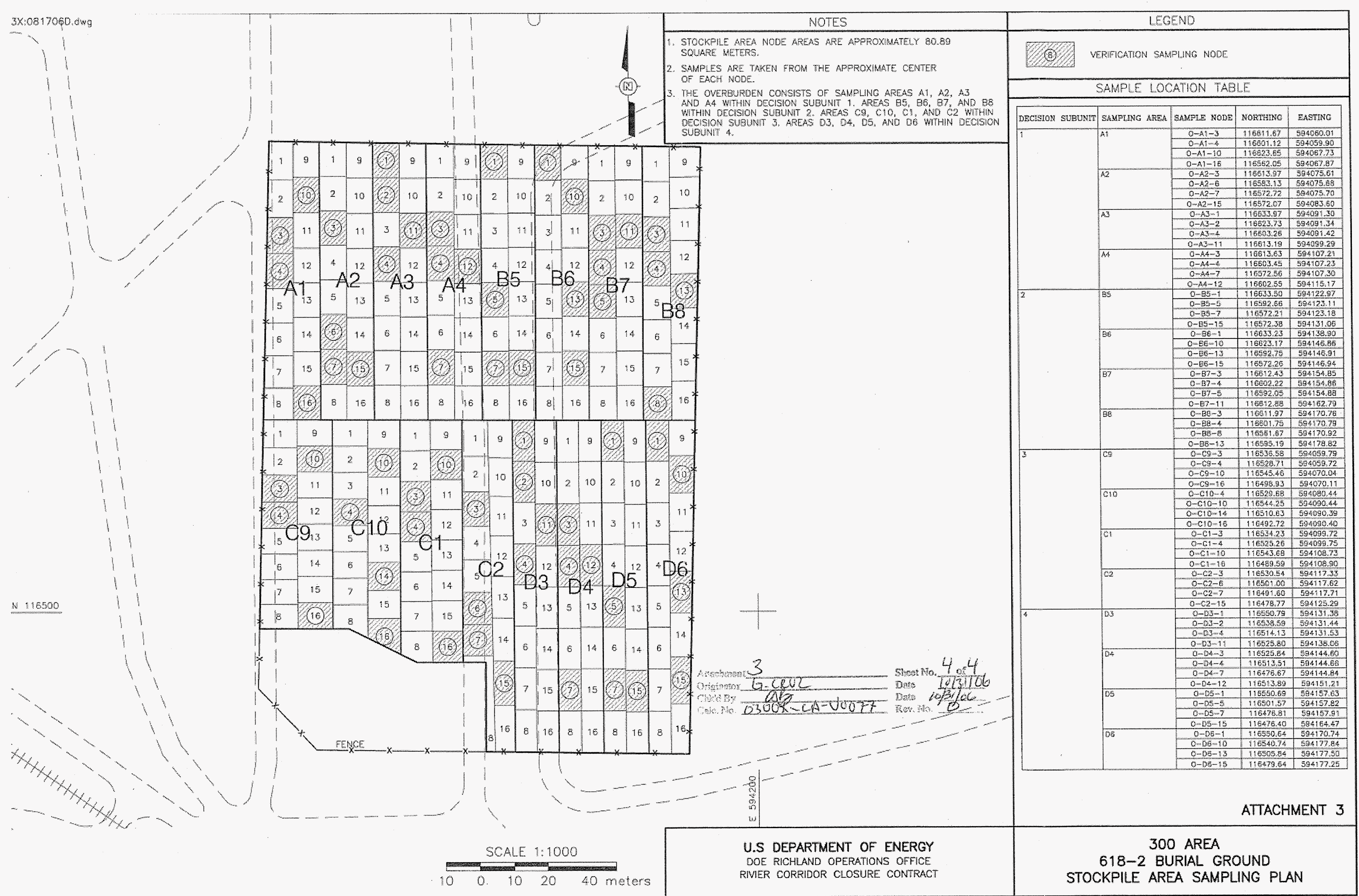


618-2 Burial Ground Cleanup Verification Model

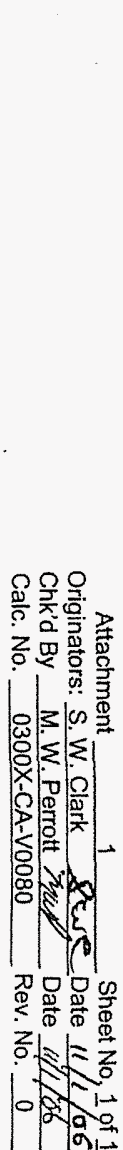

Vadose Zone Contribution to

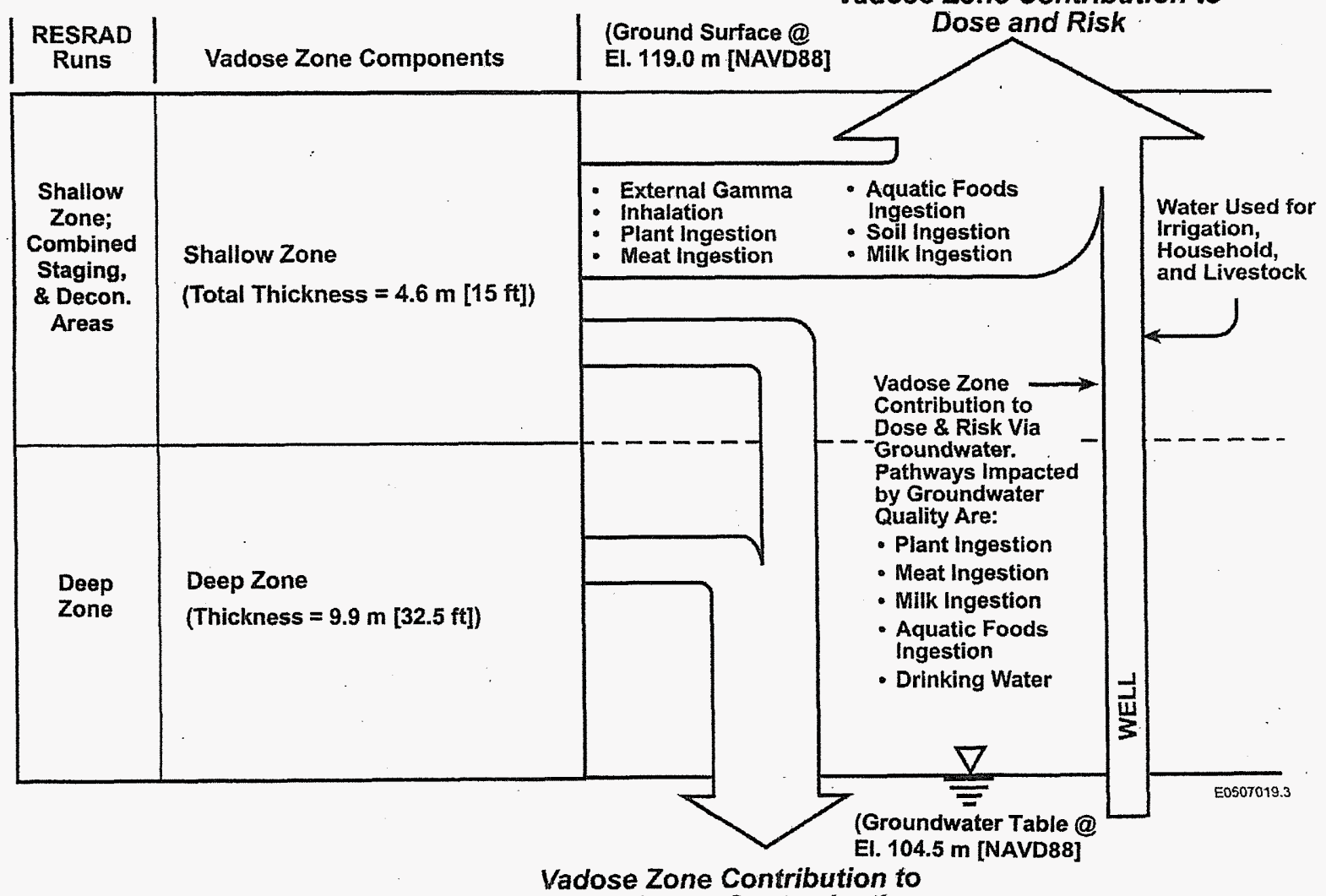

空 


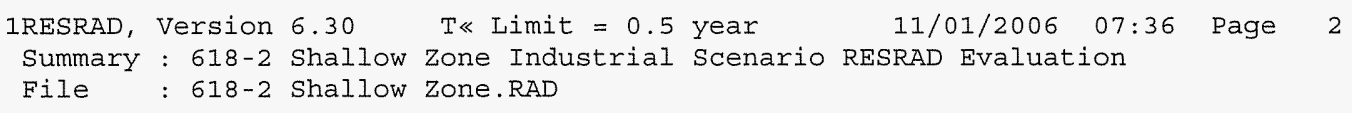

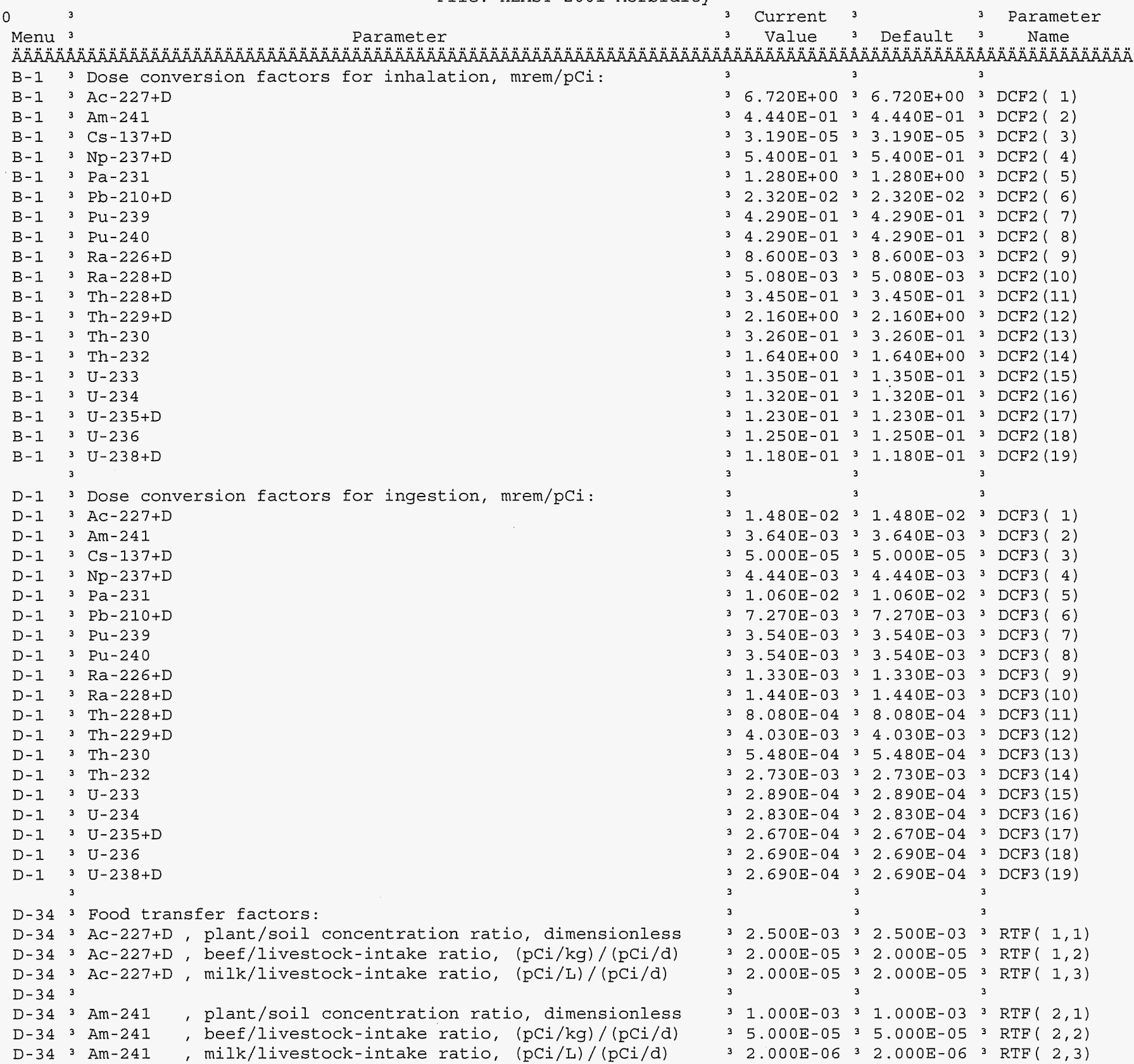




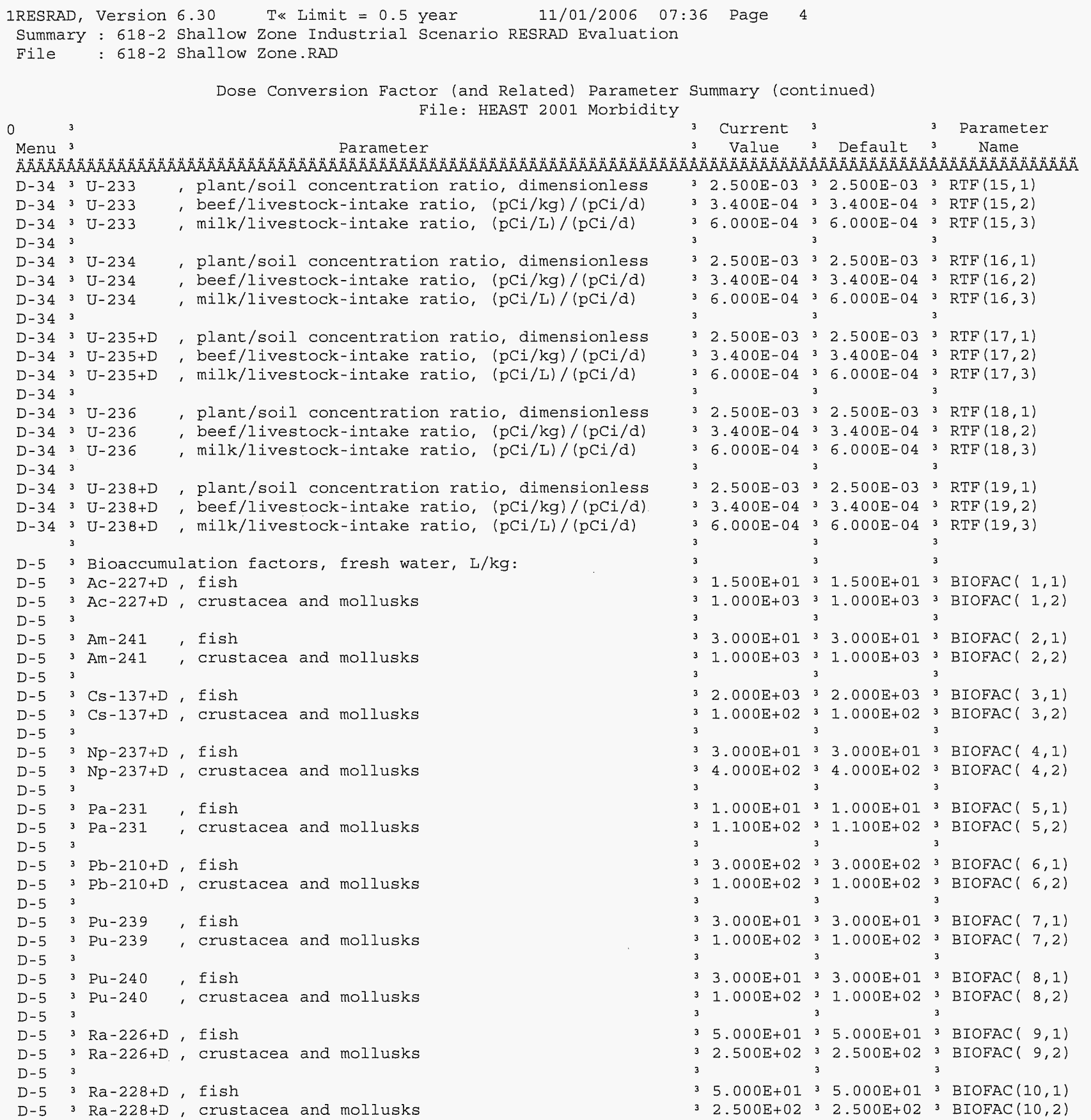


1RESRAD, Version $6.30 \quad$ T« Limit $=0.5$ year $\quad 11 / 01 / 2006 \quad 07: 36$ Page 5
Summary : $618-2$ Shallow zone Industrial Scenario RESRAD Evaluation

File : 618-2 Shallow Zone.RAD

Dose Conversion Factor (and Related) Parameter Summary (continued)

File: HEAST 2001 Morbidity

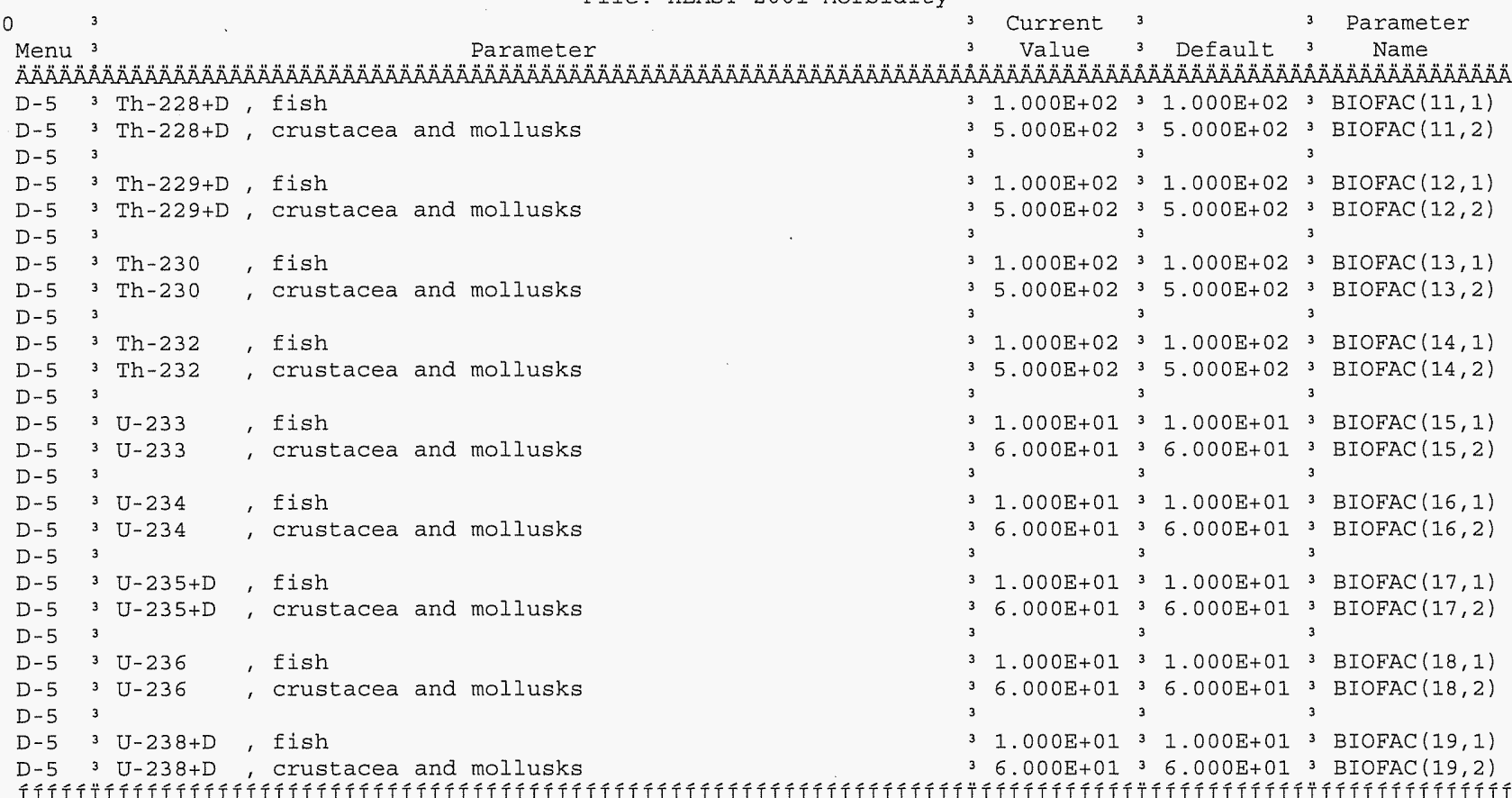


1RESRAD, Version $6.30 \quad T \&$ Limit $=0.5$ year $\quad 11 / 01 / 2006 \quad 07: 36$ Page 6 Summary : 618 -2 Shallow Zone Industrial Scenario RESRAD Evaluation

File : 618-2 Shallow Zone. RAD Menu

Site-Specific Parameter Summary 3 User 3 3 Used by RESRAD 3 Parameter

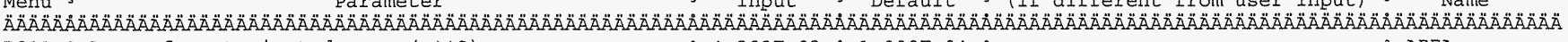
R011 3 Area of contaminated zone $\left(\mathrm{m}^{\star *} 2\right)$ R011 3 Thickness of contaminated zone (m)

R011 3 Length parallel to aquifer flow $(\mathrm{m})$

R011 3 Basic radiation dose limit (mrem/yr)

R011 3 Time since placement of material (yr)

R011 ${ }^{3}$ Times for calculations (yr)

R011 3 Times for calculations (yr)

R011 3 Times for calculations $(\mathrm{yr})$

R011 3 Times for calculations $(y r)$

R011 3 Times for calculations $(y x)$

R011 3 Times for calculations ( $\mathrm{yr}$ )

R011 3 Times for calculations ( $\mathrm{yr}$ )

R011 3 Times for calculations $(\mathrm{yr})$

R011 3 Times for calculations (yr) 3

R012 3 Initial principal radionuclide (pCi/g)

R012 3 Initial principal radionuclide (pCi/g)

R012 3 Initial principal radionuclide (pCi/g)

R012 3 Initial principal radionuclide ( $\mathrm{pCi} / \mathrm{g}$ )

R012 3 Initial principal radionuclide ( $\mathrm{pCi} / \mathrm{g}$ ):

R012 3 Initial principal radionuclide ( $\mathrm{pCi} / \mathrm{g}$ )

$\mathrm{R} 0123$ Initial principal radionuclide (pCi/g):

R012 3 Concentration in groundwater (pCi/L)

R012 3 Concentration in groundwater (pCi/L)

R012 3 Concentration in groundwater

R012 3 Concentration in groundwater

$(\mathrm{pCi} / \mathrm{L})$

Pu-239 3 not used $30.000 \mathrm{E}+00$

$34.202 \mathrm{E}+03 \quad 3 \quad 1.000 \mathrm{E}+04$

$34.600 E+0032.000 E+00$

3 not used $31.000 \mathrm{E}+02$

$31.500 \mathrm{E}+01 \quad 3 \quad 2.500 \mathrm{E}+01^{3}$

$30.000 E+0030.000 E+00$

$31.000 \mathrm{E}+00^{3} \quad 1.000 \mathrm{E}+00^{3}$

$33.000 \mathrm{E}+00^{3} 3.000 \mathrm{E}+00^{3}$

$31.000 E+0131.000 E+01$

$31.200 E+01 \quad 3 \quad 3.000 E+01$

$3.800 \mathrm{E}+013 \mathrm{3}, 000 \mathrm{~B}+01.000 \mathrm{E}+02$

$31.000 \mathrm{E}+02 \quad 3 \quad 3.000 \mathrm{E}+023$

$33.000 \mathrm{E}+02 \quad 3 \quad 1.000 \mathrm{E}+03$

$31.000 \mathrm{E}+03 \quad 3 \quad 0.000 \mathrm{E}+00$

3 not used $30.000 E+00^{3}$

$A m-241 \quad 3 \quad 5.400 E-01 \quad 3 \quad 0.000 E+003$

$\mathrm{Cs}-13731.500 \mathrm{E}+00^{3} 0.000 \mathrm{E}+00^{3}$

$\mathrm{Pu}-23934.200 \mathrm{E}+00^{3} 0.000 \mathrm{E}+00^{3}$

Pu-240 $31.000 E+0030.000 E+00$

$-234 \quad 3 \quad 9.300 \mathrm{E}-0130.000 \mathrm{E}+00$

$\mathrm{U}-23536.200 \mathrm{E}-0230.000 \mathrm{E}+00$

$\mathrm{U}-23831.070 \mathrm{E}+00 \quad 3 \quad 0.000 \mathrm{E}+00$

Am-241 3 not used $30.000 E+00$

Cs-137 3 not used $30.000 E+00^{3}$

R012 3 Concentration in groundwater $(\mathrm{pCi} / \mathrm{L}):\left(\mathrm{U}-235{ }_{3}\right.$ not used $30.000 \mathrm{E}+00_{3}$

R012 3 Concentration in groundwatex (pCi/L): U-238 3 not used $30.000 \mathrm{E}+00^{3}$ 3

R013 3 Cover depth (m)

R013 3 Density of cover material $(\mathrm{g} / \mathrm{cm} * * 3)$

R013 3 Cover depth exosion rate $(\mathrm{m} / \mathrm{Yr})$

R013 3 Density of contaminated zone $(\mathrm{g} / \mathrm{cm} * * 3)$

R.013 3 Contaminated zone erosion rate $(\mathrm{m} / \mathrm{yr})$

R013 3 Contaminated zone total porosity

R013 3 Contaminated zone field capacity

$30.000 \mathrm{E}+00^{3} 0.000 \mathrm{E}+00^{3}$

3 not used $31.500 \mathrm{E}+00$

3 not used $31.000 \mathrm{E}-03$,

$1.600 \mathrm{E}+00 \quad 3 \quad 1.500 \mathrm{E}+003$

$31.000 \mathrm{E}-033^{3} 1.000 \mathrm{E}-03^{3}$

$33.000 \mathrm{E}-01 \quad 3 \quad 4.000 \mathrm{E}-01$

$32.500 \mathrm{E}-0132.000 \mathrm{E}-01$

R013 3 Contaminated zone hydraulic conductivity $(\mathrm{m} / \mathrm{Yr}) \quad 32.200 \mathrm{E}-0331.000 \mathrm{E}+01$

R013 3 Contaminated zone b parameter

R013 3 Average annual wind speed (m/sec)

3. $1.500 \mathrm{E}+0135.300 \mathrm{E}+00$

$3.400 \mathrm{E}+0032.000 \mathrm{E}+003$

3 not used $38.000 \mathrm{E}+00^{3}$

$39.100 E-01 \quad 3 \quad 5.000 E-013$

$31.524 \mathrm{E}-0131.000 \mathrm{E}+00$

$30.000 \mathrm{E}+00 \quad 3 \quad 2.000 \mathrm{E}-013$

3 overhead 3 overhead

3 2.000E-01 3 2.000E-01

3 not used $31.000 \mathrm{E}+06^{3}$

3 not used 3 1.000E-03 3

3 not used $31.500 \mathrm{E}+00^{3}$

3 AREA

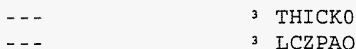

3 BRDI

-.

$\begin{array}{ll}\ldots & \mathrm{TI} \\ \ldots & \mathrm{T}(2)\end{array}$

$\begin{array}{lll}\ldots & \mathrm{T}(2) \\ \ldots & { }_{3} \mathrm{~T}(3)\end{array}$

$\ldots \quad 3 \mathrm{~T}(4)$

$\ldots 3 \mathrm{~T}(5)$

-.. $3 \mathrm{~T}(6)$

$\begin{array}{lll}\ldots & \\ \ldots & & T\end{array}(7)$

$\begin{array}{ll}\ldots & \mathrm{T}(8)\end{array}$

-. 3 T( 9 )

-. $3 \mathrm{~T}(10)$

$\ldots \quad 3 S 1(2)$

- $\quad 3$ SI $(3)$

${ }^{3} \operatorname{SI}(7)$

${ }^{3} S 1(8)$

${ }^{3} \mathrm{SI}(16)$

-.- 3 SI(17)

-. $\quad 3$ SI(19)

${ }^{3}$ WI $(2)$

$-\cdots$

3 W1 $(7)$

-.- 3 W1 ( 8)

3 W1 $(16)$

3 W1 (17)

-. 3 W1(19)

3 COVERO

-.. 3 DENSCV

3 VCV

3 DENSCZ

$3 \mathrm{VCZ}$

3 TPCZ

3 FCCZ

-.. $3 \mathrm{HCCZ}$

$3 \mathrm{BCZ}$

-..

3 EVAPTR

3 PRECI

$\begin{array}{ll}3 & \text { RI } \\ -.- & 3 \\ \text { IDITCH }\end{array}$

3 RUNOF

3 WAREA

Romberg failures occurred ${ }_{3}^{3}$ EPS

3 DENSAQ

$-$

R014 3 Density of saturated zone $(\mathrm{g} / \mathrm{cm} * * 3)$ 


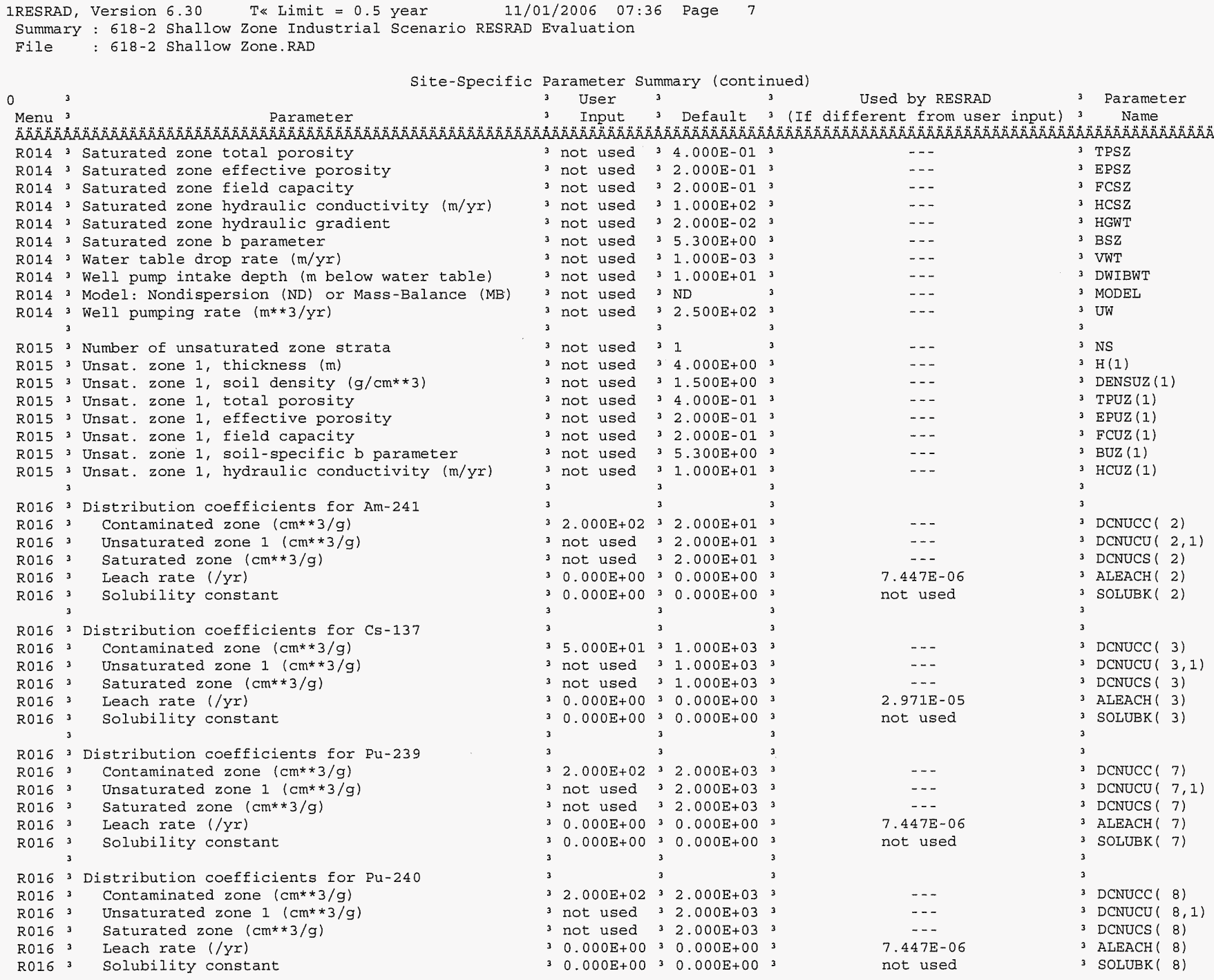




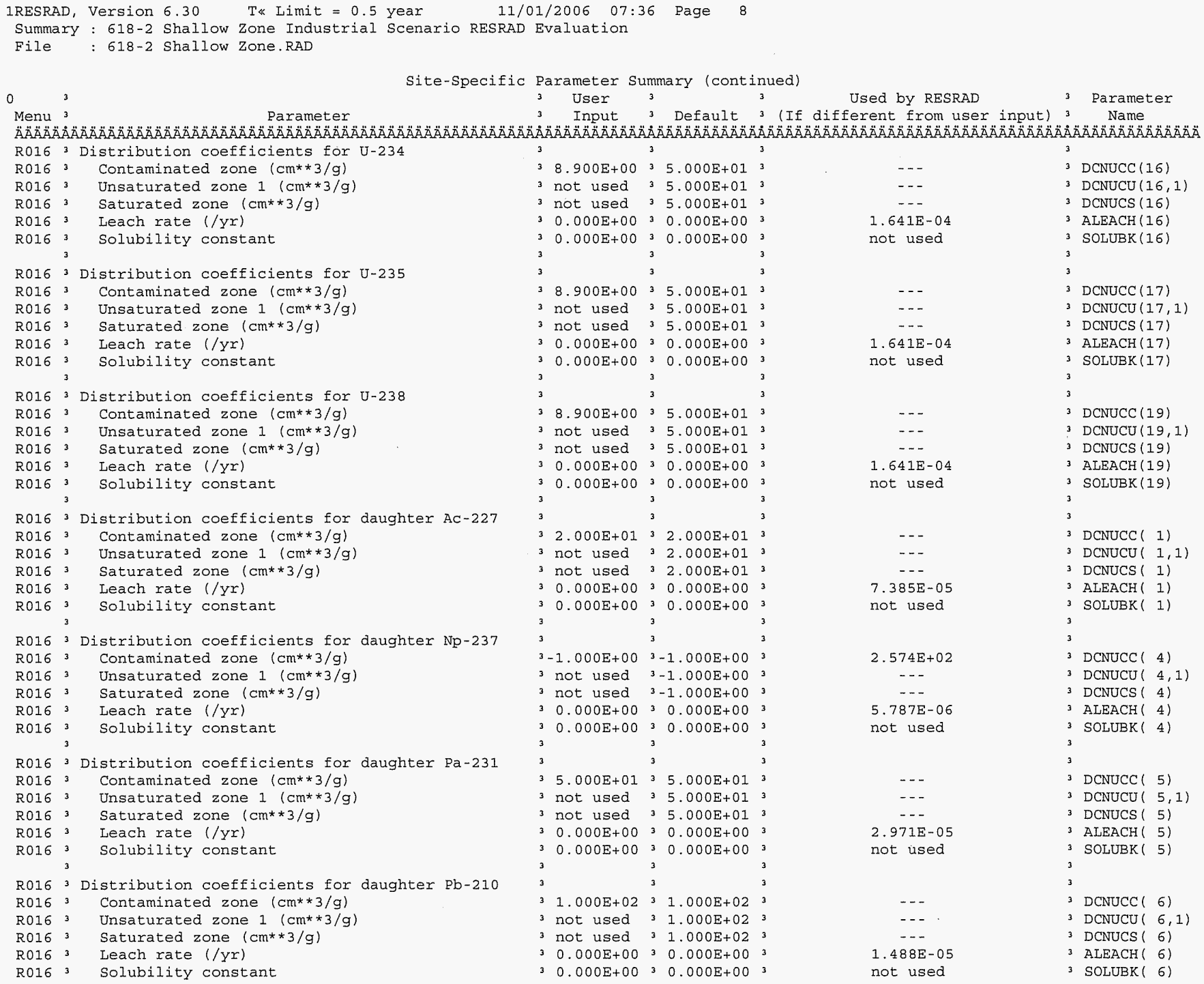


1RESRAD, Version $6.30 \quad T$ Limit $=0.5$ year $11 / 01 / 2006 \quad 07: 36$ Page 9

Summary : 618-2 Shallow Zone Industrial Scenario RESRAD Evaluation

File: 618-2 shallow zone.RAD

\begin{tabular}{|c|c|c|c|c|c|c|c|c|c|}
\hline \multirow{3}{*}{\multicolumn{2}{|c|}{$0 \quad{ }^{3}$}} & Site-Specific & \multirow{2}{*}{\multicolumn{6}{|c|}{ Parameter Summary (continued) }} & \multirow{2}{*}{ Parameter } \\
\hline & & & & & & & & & \\
\hline & & Parameter & Input & \multirow{2}{*}{3} & \multirow{2}{*}{$\begin{array}{l}\text { Default } \\
\ddot{A} \ddot{A} \ddot{A} \ddot{A} \ddot{A} \ddot{A} \ddot{A} \ddot{A} \ddot{A} \ddot{A} \ddot{A}\end{array}$} & \multirow{2}{*}{\multicolumn{2}{|c|}{ (If different from user input) }} & & \multirow{2}{*}{$\begin{array}{c}\text { Name } \\
\ddot{A} \ddot{A} \ddot{A} \ddot{A} \ddot{A} \ddot{A} \ddot{A} \ddot{A} \ddot{A} \ddot{A} \ddot{A} \ddot{A} \ddot{A} \ddot{A}\end{array}$} \\
\hline & & ÄН̈Ä & 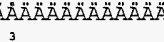 & & & & & & \\
\hline R016 & 3 & Distribution coefficients for daughter $R a-226$ & 3 & 3 & & 3 & & 3 & \\
\hline R016 & 3 & Contaminated zone $(\mathrm{cm} * * 3 / \mathrm{g})$ & $37.000 \mathrm{E}+01$ & 3 & $7.000 \mathrm{E}+01$ & ${ }^{3}$ & - - - & 3 & DCNUCC ( 9) \\
\hline $\mathrm{R} 016$ & ${ }^{3}$ & Unsaturated zone $1(\mathrm{~cm} * * 3 / \mathrm{g})$ & 3 not used & 3 & $7.000 E+01$ & 3 & --- & 3 & DCNUCU $(9,1)$ \\
\hline R016 & 3 & Saturated zone $(\mathrm{cm} * * 3 / \mathrm{g})$ & 3 not used & 3 & $7.000 \mathrm{E}+01$ & 3 & -.- & 3 & DCNUCS ( 9) \\
\hline R016 & 3 & Leach rate $(/ y r)$ & $30.000 E+00$ & 3 & $0.000 \mathrm{E}+00$ & 3 & $2.124 \mathrm{E}-05$ & 3 & AIEACH ( 9) \\
\hline \multirow[t]{2}{*}{ R016 } & 3 & Solubility constant & $30.000 E+00$ & 3 & $0.000 E+00$ & 3 & not used & 3 & SOLUBK( 9) \\
\hline & 3 & & 3 & 3 & & 3 & & 3 & \\
\hline R016 & 3 & Distribution coefficients for daughter Ra-228 & 3 & 3 & & 3 & & 3 & \\
\hline $\mathrm{R} 016$ & 3 & Contaminated zone $\left(\mathrm{cm}^{\star *} 3 / \mathrm{g}\right)$ & $37.000 E+01$ & 3 & $7.000 \mathrm{E}+01$ & 3 & -- & 3 & $\operatorname{DCNUCC}(10)$ \\
\hline R016 & 3 & Unsaturated zone $1(\mathrm{~cm} * 3 / \mathrm{g})$ & 3 not used & 3 & $7.000 \mathrm{E}+01$ & 3 & $\cdots$ & 3 & $\operatorname{DCNUCU}(10,1)$ \\
\hline $\mathrm{R} 016$ & 3 & Saturated zone $(\mathrm{cm} * * 3 / \mathrm{g})$ & 3 not used & 3 & $7.000 E+01$ & 3 & --- & 3 & $\operatorname{DCNUCS}(10)$ \\
\hline R016 & 3 & Leach rate $(/ y r)$ & $30.000 E+00$ & 3 & $0.000 E+00$ & 3 & $2.124 E-05$ & 3 & ALEACH $(10)$ \\
\hline \multirow[t]{2}{*}{$\mathrm{R} 016$} & 3 & Solubility constant & ${ }^{3} 0.000 \mathrm{E}+00$ & 3 & $0.000 \mathrm{E}+00$ & 3 & not used & 3 & SOLUBK (10) \\
\hline & 3 & & 3 & 3 & & 3 & . & 3 & \\
\hline $\mathrm{R} 016$ & 3 & Distribution coefficients for daughter Th-228 & 3 & 3 & & 3 & & 3 & \\
\hline R016 & 3 & Contaminated zone $(\mathrm{cm} * * 3 / \mathrm{g})$ & $32.000 \mathrm{E}+02$ & 3 & $6.000 \mathrm{E}+04$ & 3 & 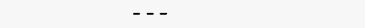 & 3 & DCNUCC (11) \\
\hline $\mathrm{R} 016$ & 3 & Unsaturated zone $1(\mathrm{~cm} * * 3 / \mathrm{g})$ & 3 not used & 3 & $6.000 E+04$ & 3 & -- & 3 & DCNUCU $(11,1)$ \\
\hline R016 & 3 & Saturated zone $(\mathrm{cm} * * 3 / \mathrm{g})$ & 3 not used & 3 & $6.000 \mathrm{E}+04$ & 3 & -. - & 3 & DCNUCS (11) \\
\hline R016 & 3 & Leach rate $(/ \mathrm{yr})$ & $30.000 \mathrm{E}+00$ & 3 & $0.000 \mathrm{E}+00$ & 3 & $7.447 \mathrm{E}-06$ & 3 & ALEACH (11) \\
\hline \multirow[t]{2}{*}{ R016 } & 3 & Solubility constant & $30.000 \mathrm{E}+00$ & 3 & $0.000 \mathrm{E}+00$ & 3 & not used & 3 & SOLUBK (11) \\
\hline & 3 & & 3 & 3 & & 3 & & 3 & \\
\hline $\mathrm{R} 016$ & 3 & Distribution coefficients for daughter Th-229 & 3 & 3 & & 3 & & 3 & \\
\hline R016 & ${ }^{3}$ & Contaminated zone $\left(\mathrm{cm}^{* * 3 / \mathrm{g})}\right.$ & $32.000 E+02$ & 3 & $6.000 \mathrm{E}+04$ & 3 & $-\cdot$ & 3 & DCNUCC (12) \\
\hline R016 & 3 & Unsaturated zone $1\left(\mathrm{~cm}^{*} * 3 / \mathrm{g}\right)$ & 3 not used & 3 & $6.000 \mathrm{E}+04$ & 3 &.-- & 3 & $\operatorname{DCNUCU}(12,1)$ \\
\hline R016 & 3 & Saturated zone $(\mathrm{cm} * * 3 / \mathrm{g})$ & 3 not used & 3 & $6.000 \mathrm{E}+04$ & 3 & $\cdots$ & 3 & DCNUCS (12) \\
\hline R016 & 3 & Leach rate (/yr) & $30.000 E+00$ & 3 & $0.000 \mathrm{E}+00$ & 3 & $7.447 \mathrm{E}-06$ & 3 & ALEACH (12) \\
\hline \multirow[t]{2}{*}{ R016 } & 3 & Solubility constant & $30.000 E+00$ & 3 & $0.000 E+00$ & 3 & not used & 3 & SOLUBK (12) \\
\hline & 3 & & 3 & 3 & & 3 & & 3 & \\
\hline R016 & 3 & Distribution coefficients for daughter Th-230 & 3 & 3 & & 3 & & ${ }^{3}$ & \\
\hline R016 & 3 & Contaminated zone $(\mathrm{cm} * * 3 / \mathrm{g})$ & $32.000 E+02$ & 3 & $6.000 \mathrm{E}+04$ & 3 & -.. & 3 & DCNUCC (13) \\
\hline R.016 & 3 & Unsaturated zone $1\left(\mathrm{~cm}^{*} * 3 / \mathrm{g}\right)$ & 3 not used & 3 & $6.000 E+04$ & 3 & -. - & 3 & $\operatorname{DCNUCU}(13,1)$ \\
\hline R016 & 3 & Saturated zone $(\mathrm{cm} * * 3 / \mathrm{g})$ & 3 not used & 3 & $6.000 \mathrm{E}+04$ & 3 & $\cdots$ & 3 & DCNUCS (13) \\
\hline R016 & 3 & Leach rate $(/ y x)$ & $30.000 \mathrm{E}+00$ & 3 & $0.000 \mathrm{E}+00$ & 3 & $7.447 \mathrm{E}-06$ & 3 & ALEACH (13) \\
\hline \multirow[t]{2}{*}{ R016 } & 3 & Solubility constant & $30.000 \mathrm{E}+00$ & 3 & $0.000 \mathrm{E}+00$ & 3 & not used & 3 & SOLUBK (13) \\
\hline & 3 & & 3 & 3 & & 3 & & 3 & \\
\hline R016 & 3 & Distribution coefficients for daughter Th-232 & 3 & 3 & & 3 & & 3 & \\
\hline R016 & 3 & Contaminated zone $(\mathrm{cm} * * 3 / \mathrm{g})$ & ${ }^{3} 2.000 \mathrm{E}+02$ & 3 & $6.000 E+04$ & 3 & -. & ${ }^{3}$ & DCNUCC (14) \\
\hline R016 & 3 & Unsaturated zone $1(\mathrm{~cm} * * 3 / \mathrm{g})$ & 3 not used & 3 & $6.000 E+04$ & 3 & ..- & ${ }^{3}$ & $\operatorname{DCNUCU}(14,1)$ \\
\hline R016 & 3 & Saturated zone $(\mathrm{cm} * * 3 / \mathrm{g})$ & 3 not used & 3 & $6.000 E+04$ & 3 & -.. & ${ }^{3}$ & DCNUCS (14) \\
\hline R016 & 3 & Leach rate (/yr) & $30.000 E+00$ & 3 & $0.000 E+00$ & 3 & $7.447 \mathrm{E}-06$ & 3 & $\operatorname{ALEACH}(14)$ \\
\hline \multirow[t]{2}{*}{ R016 } & 3 & Solubility constant & $30.000 \mathrm{E}+00$ & 3 & $0.000 E+00$ & 3 & not used & 3 & SOLUBK (14) \\
\hline & 3 & & 3 & 3 & & 3 & & 3 & \\
\hline R016 & 3 & Distribution coefficients for daughter U-233 & 3 & 3 & & 3 & & 3 & \\
\hline R016 & 3 & Contaminated zone $(\mathrm{cm} * * 3 / \mathrm{g})$ & $38.900 \mathrm{E}+00$ & 3 & $5.000 \mathrm{E}+01$ & 3 & $-\cdots$ & 3 & DCNUCC (15) \\
\hline R016 & 3 & Unsaturated zone $1(\mathrm{~cm} * * 3 / \mathrm{g})$ & 3 not used & 3 & $5.000 \mathrm{E}+01$ & 3 & $\cdots$ & 3 & $\operatorname{DCNUCU}(15,1)$ \\
\hline R016 & ${ }^{3}$ & Saturated zone $(\mathrm{cm} * * 3 / \mathrm{g})$ & 3 not used & 3 & $5.000 \mathrm{E}+01$ & 3 & $-\cdots$ & 3 & DCNUCS (15) \\
\hline $\mathrm{R} 016$ & 3 & Leach rate (/yr) & $30.000 \mathrm{E}+00$ & 3 & $0.000 \mathrm{E}+00$ & 3 & $1.641 \mathrm{E}-04$ & 3 & ALEACH (15) \\
\hline R016 & 3 & Solubility constant & $30.000 \mathrm{E}+00$ & 3 & $0.000 \mathrm{E}+00$ & 3 & not used & 3 & SOLUBK (15) \\
\hline
\end{tabular}


IRESRAD, Version $6.30 \quad T \ll$ Limit $=0.5$ year $\quad 11 / 01 / 2006 \quad 07: 36$ Page 10 Summary : 618-2 Shallow Zone Industrial Scenario RESRAD Evaluation

File : 618-2 Shallow Zone.RAD

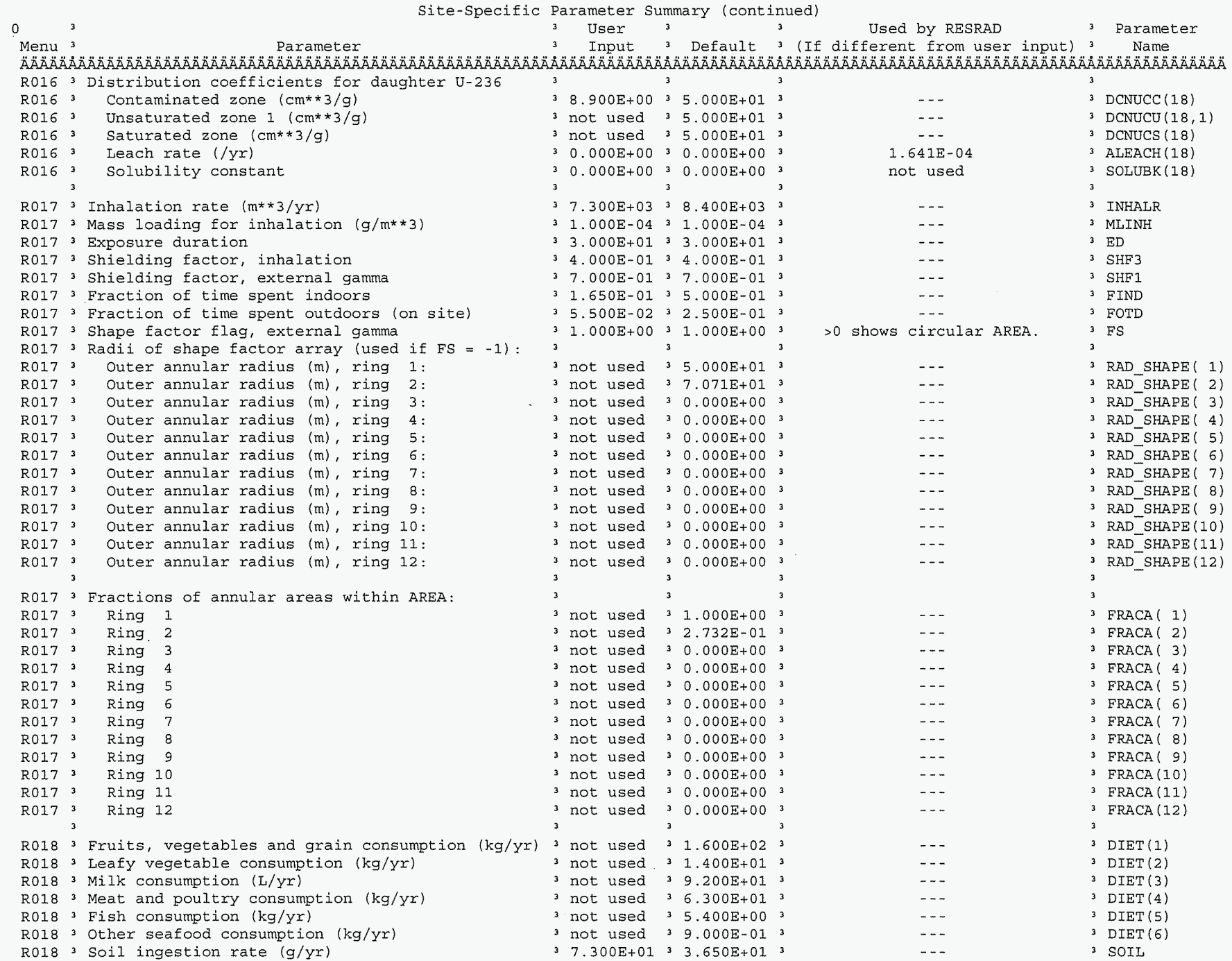




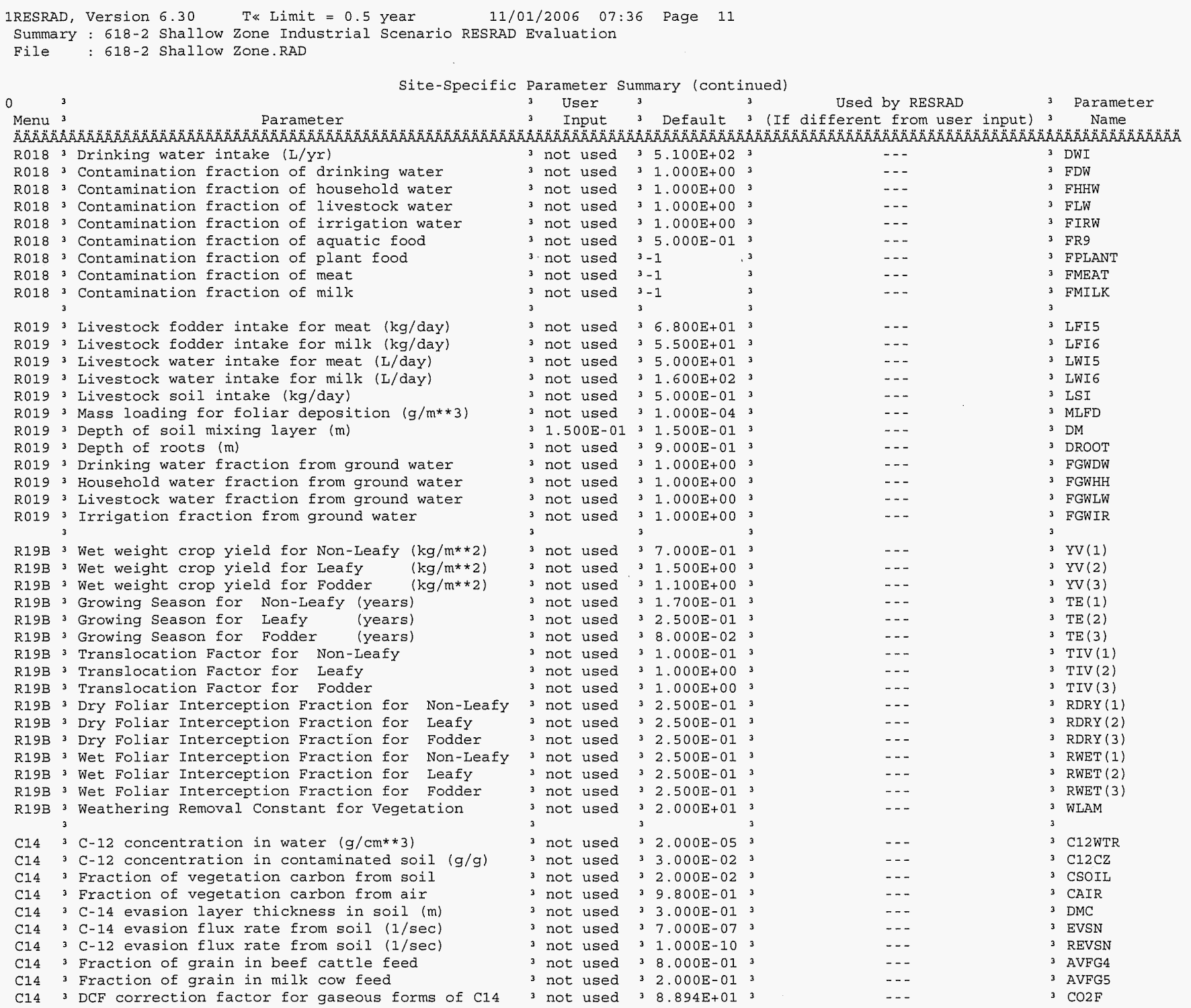


1RESRAD, Version $6.30 \quad T \&$ Limit $=0.5$ year $\quad 11 / 01 / 2006 \quad 07: 36$ Page 12

Summary : 618-2 Shallow Zone Industrial Scenario RESRAD Evaluation

File : 618-2 Shallow Zone.RAD

0

Site-Specific Parameter Summary (continued)

Menu

3 User 3

Used bY RESRAD

3 Parameter

Parameter ${ }^{3}$ Input ${ }^{3}$ Default ${ }^{3}$ (If different from user input) ${ }^{3} \quad$ Name

STOR 3 Storage times of contaminated foodstuffs (days) :

STOR 3 Fruits, non-leafy vegetables, and grain

STOR 3 Leafy vegetables

STOR ${ }^{3}$ Milk

STOR ${ }^{3}$ Meat and poultry

STOR 3 Fish

STOR ${ }^{3}$ Crustacea and mollusks

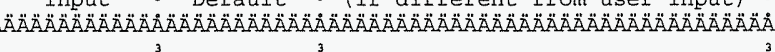

$\begin{array}{ll}\text { STOR }^{3} & \text { Crustacea } \\ \text { STOR } & 3 \\ \text { Well wate }\end{array}$

STOR ${ }^{3}$ Surface water

STOR 3 Livestock fodder

R021 3 Thickness of building foundation (m)

$3 \quad 1.400 \mathrm{E}+01 \quad 3 \quad 1.400 \mathrm{E}+01^{3}$

-

$31.000 E+0031.000 E+00$

$-\cdot$

3 STOR T (1)

$31.000 \mathrm{E}+00^{3} 1.000 \mathrm{E}+00^{3}$

$32.000 E+0132.000 E+013$

$37.000 E+0037.000 E+003$

$37.000 E+0037.000 E+00$

$31.000 \mathrm{E}+00^{3} 1.000 \mathrm{E}+00^{3}$

$31.000 \mathrm{E}+00^{3} 1.000 \mathrm{E}+00^{3}$

${ }^{3} 4.500 \mathrm{E}+01^{3} 4.500 \mathrm{E}+01^{3}$

3 not used 3 1.500E-01

3 not used $32.400 \mathrm{E}+00$

3 not used $34.000 E^{3} 01^{3}$

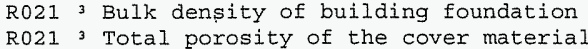

R021 3 Total porosity of the building foundation

3 not used 3 1.000E-01

3 not used $35.000 E-023$

R021 3 Volumetric water content of the foundation

R021 3 Diffusion coefficient for radon gas $(\mathrm{m} / \mathrm{sec})$ :

R021 3 in cover material

R021 3 in foundation material

RO21 3 in contaminated zone soil

R021 3 Radon vertical dimension of mixing (m)

R021 3 Average building air exchange rate (1/hr)

not used $32.000 E_{-06}^{3}$

$3.000 E-023$

3 not used $33.000 E-07^{3}$

3 not used $32.000 \mathrm{E}-06^{3}$

3 not used $32.000 E+00$

3 not used $35.000 \mathrm{E}-01$

3 not used $32.500 E+00^{3}$

3 not used $30.000 \mathrm{E}+00^{3}$

3 not used $3-1.000 \mathrm{E}+00^{3}$

3 not used $32.500 \mathrm{E}-013$

R021 3 Building depth below ground

R021 3 Emanating power of Rn-222 gas

TITL 3 Number of graphical time points

TITL 3 Maximum number of integration points for dose

TITL 3 Maximum number of integration points for risk

3 not used 3 1.500E-01

3

3

$64 \quad 3 \quad \ldots-2$

$3 \quad 5 \quad 3 \quad--$

Tiu Maximum number of integration points for risk

a $\quad 3$ STOR T (3)

- $\quad 3$ STOR_T (4)

3 STORT ${ }^{-}$T (5)

-.. 3 STOR T $^{-1}(6)$

... 3 STOR T $^{-}(7)$

-- 3 STORTI(8)

.. 3 STOR_T(9)

-. 3 FLOORI

3 DENSFI

3 TPCV

3 TPF

$3 \mathrm{PH} 2 \mathrm{OCV}$

3 PH2OFI

... 3 DIFCV

-..

$\begin{array}{ll}--- & 3 \text { DIFFL } \\ --- & 3 \text { DIFCZ }\end{array}$

3 HMIX

3 REXG

H HRM

3..

-.- 3 DMFI

-.- 3 EMANA (1)

3 EMANA (2)

-.. 3 NPTS

3 LYMAX

3 KYMAX

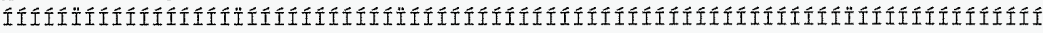

Summary of Pathway Selections

Pathway ${ }^{3}$ User Selection

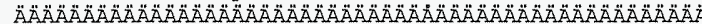

1 -- external gamma 3 active

2 - inhalation (w/o radon) ${ }^{3}$ active

3 - plant ingestion 3 suppressed

4 -- meat ingestion ${ }^{3}$ suppressed

5 -- milk ingestion 3 suppressed

6 -- aquatic foods 3 suppressed

7 - - drinking water 3 suppressed

8 -- soil ingestion 3 active

9 -- radon 3 suppressed

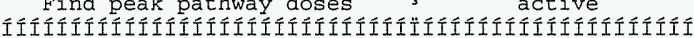




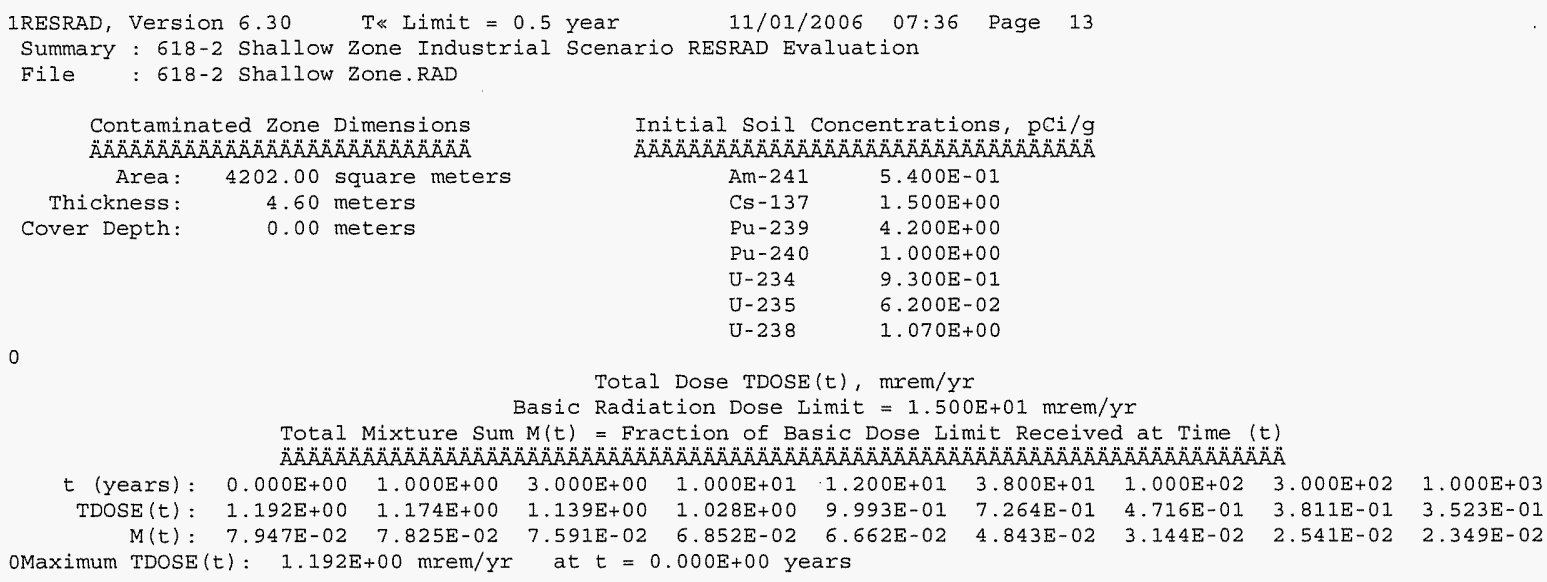

Total Dose TDOSE(t), mrem/yr Basic Radiation Dose Limit $=1.500 \mathrm{E}+01 \mathrm{mrem} / \mathrm{yr}$

Total Mixture Sum $M(t)=$ Fraction of Basic Dose Limit Received at Time $(t)$

$t$ (years) : $\begin{array}{llllllllll}0.000 \mathrm{E}+00 & 1.000 \mathrm{E}+00 & 3.000 \mathrm{E}+00 & 1.000 \mathrm{E}+01 & 1.200 \mathrm{E}+01 & 3.800 \mathrm{E}+01 & 1.000 \mathrm{E}+02 & 3.000 \mathrm{E}+02 & 1.000 \mathrm{E}+03\end{array}$ $\operatorname{TDOSE}(t)=\begin{array}{lllllllll}1.192 \mathrm{E}+00 & 1.174 \mathrm{E}+00 & 1.139 \mathrm{E}+00 & 1.028 \mathrm{E}+00 & 9.993 \mathrm{E}-01 & 7.264 \mathrm{E}-01 & 4.716 \mathrm{E}-01 & 3.811 \mathrm{E}-01 & 3.523 \mathrm{E}-01\end{array}$

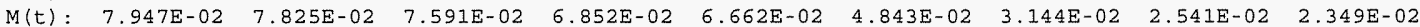
OMaximum TDOSE $(t): 1.192 \mathrm{E}+00 \mathrm{mrem} / \mathrm{Yr}$ at $t=0.000 \mathrm{E}+00$ years 


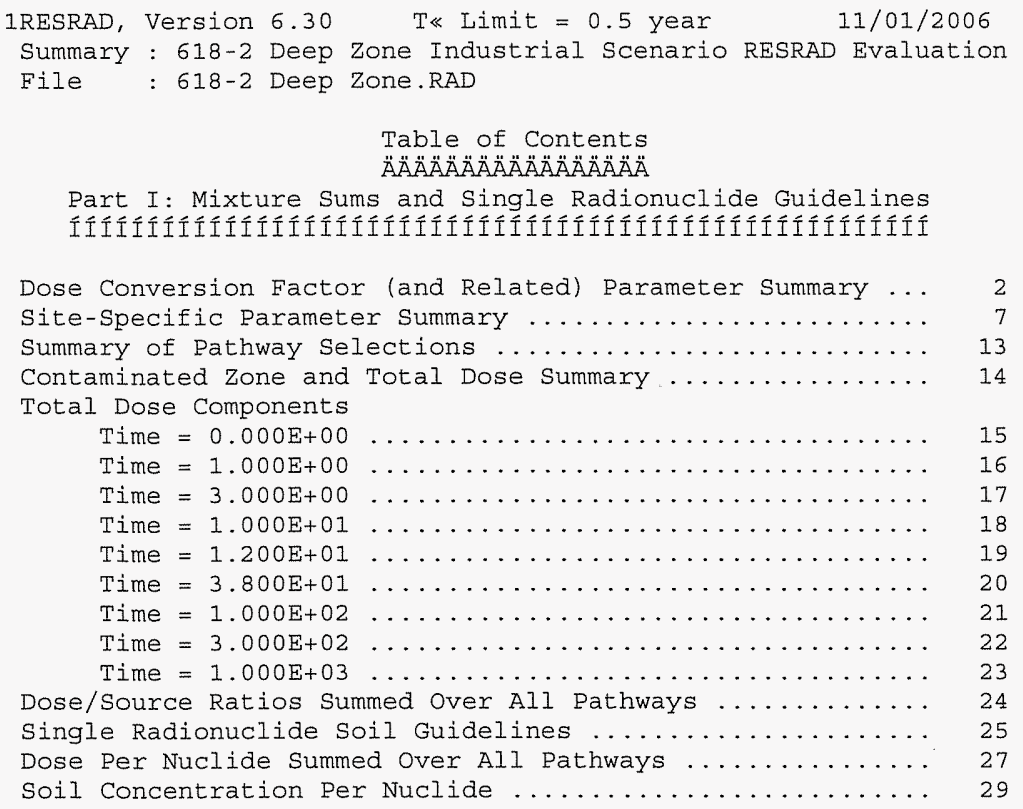




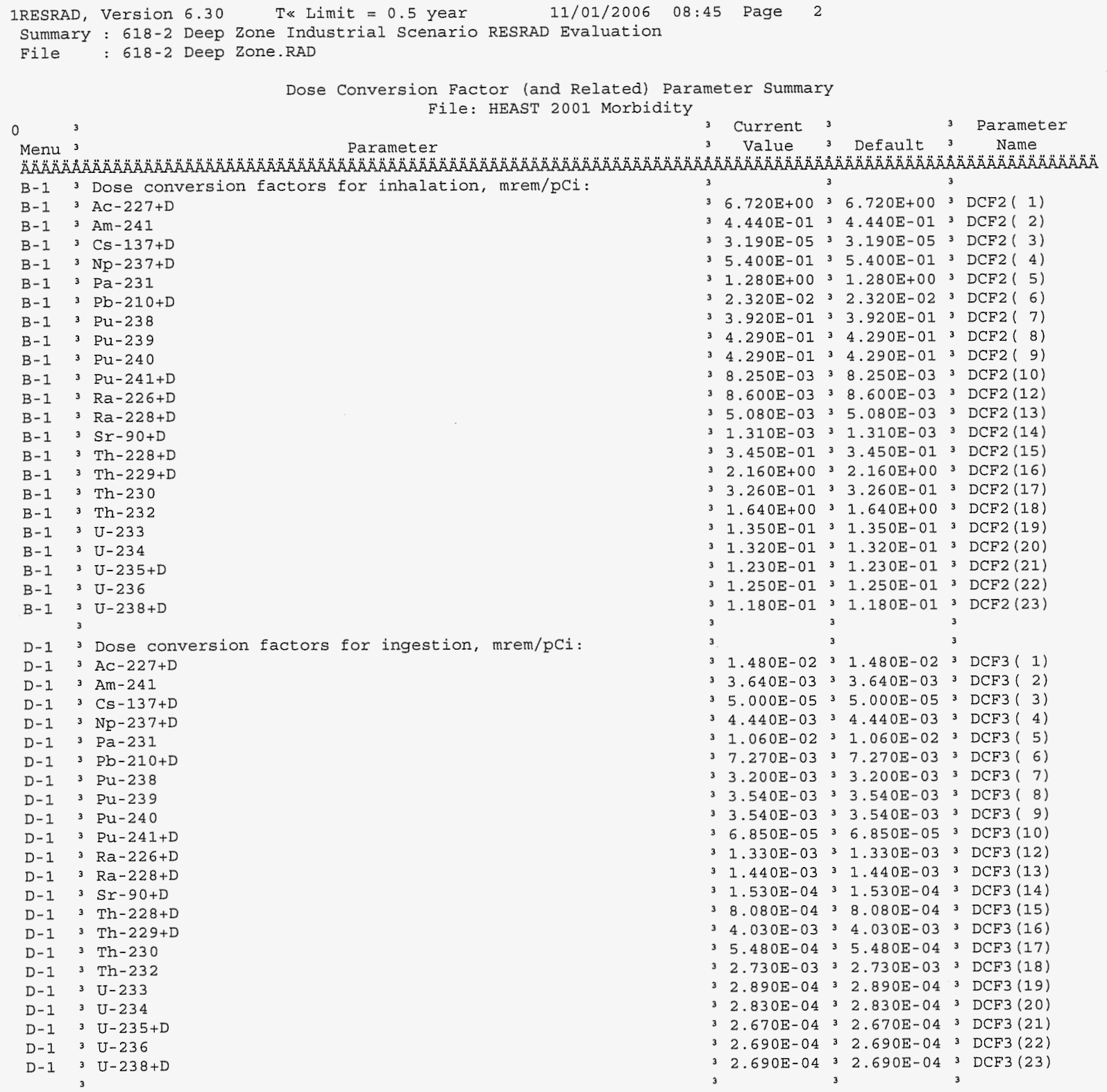




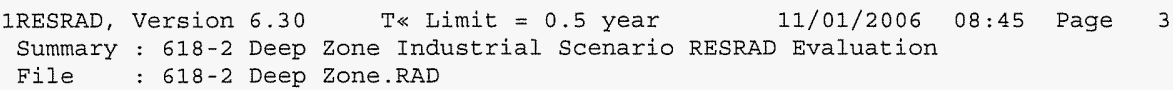

$\operatorname{Menu}^{3}$

Parameter

ter Heast 2001 Morbiaity

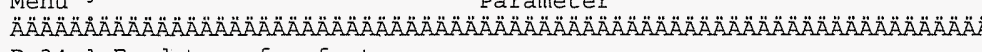

D-34 3 Food transfer factors:

$\mathrm{D}-343 \mathrm{AC}-227+\mathrm{D}$, plant/soil concentration ratio, dimensionless

$\mathrm{D}-343 \mathrm{AC}-227+\mathrm{D}$, beef/livestock-intake ratio, (pCi/ $\mathrm{kg}) /(\mathrm{pCi} / \mathrm{d})$

$\mathrm{D}-34{ }^{3} \mathrm{AC}-227+\mathrm{D}$, milk/livestock-intake ratio, $(\mathrm{pCi} / \mathrm{L}) /(\mathrm{pCi} / \mathrm{d})$

$\mathrm{D}-34^{3}$

D-34 3 Am-241, plant/soil concentration ratio, dimensionless

$\mathrm{D}-343 \mathrm{Am}-241$, beef/livestock-intake ratio, (pCi/kg)/(pCi/d)

$\mathrm{D}-34{ }^{3} \mathrm{Am}-241$, milk/livestock-intake ratio, $(\mathrm{pCi} / \mathrm{L}) /(\mathrm{pCi} / \mathrm{d})$

$\mathrm{D}-344^{3}$

D-34 3 Cs-137+D, plant/soil concentration ratio, dimensionless

$\mathrm{D}-343 \mathrm{Cs}-137+\mathrm{D}$, beef/livestock-intake ratio, (pCi/kg)/(pCi/d)

$\mathrm{D}-343 \mathrm{Cs}-137+\mathrm{D}$, milk/livestock-intake ratio, (pCi/L)/(pCi/d)

$\mathrm{D}-34{ }^{3}$

D-34 $3 \mathrm{~Np}-237+\mathrm{D}$, plant/soil concentration ratio, dimensionless

$\mathrm{D}-343 \mathrm{~Np}-237+\mathrm{D}$, beef/livestock-intake ratio, $(\mathrm{pCi} / \mathrm{kg}) /(\mathrm{pCi} / \mathrm{d})$

$\mathrm{D}-343 \mathrm{~Np}-237+\mathrm{D}$, $\mathrm{milk} / \mathrm{livestock-intake} \mathrm{ratio,}(\mathrm{pCi} / \mathrm{L}) /(\mathrm{pCi} / \mathrm{d})$

$\mathrm{D}-344^{3}$

$\mathrm{D}-34^{3} \mathrm{~Pa}-231$, plant/soil concentration ratio, dimensionless

$\mathrm{D}-343 \mathrm{~Pa}-231$, beef/livestock-intake ratio, (pCi/ $\mathrm{kg}) /(\mathrm{pCi} / \mathrm{d})$

$\mathrm{D}-343 \mathrm{~Pa}-231$, $\mathrm{milk} /$ livestock-intake ratio, (pCi/L)/(pCi/d)

$\mathrm{D}-34{ }^{3}$

$\mathrm{D}-343 \mathrm{~Pb}-210+\mathrm{D}$, plant/soil concentration ratio, dimensionless

$\mathrm{D}-343 \mathrm{~Pb}-210+\mathrm{D}$, beef/livestock-intake ratio, $(\mathrm{pCi} / \mathrm{kg}) /(\mathrm{pCi} / \mathrm{d})$

$\mathrm{D}-343 \mathrm{~Pb}-210+\mathrm{D}$, milk/livestock-intake ratio, $(\mathrm{pCi} / \mathrm{L}) /(\mathrm{pCi} / \mathrm{d})$

$\mathrm{D}-34 \quad 3$

$\mathrm{D}-34^{3} \mathrm{Pu}-238$

$\mathrm{D}-343 \mathrm{Pu}-238$

plant/soil concentration ratio, dimensionless

, beef/livestock-intake ratio, $(\mathrm{pCi} / \mathrm{kg}) /(\mathrm{pCi} / \mathrm{d})$

$\mathrm{D}-34{ }^{3} \mathrm{Pu}-238$, milk/livestock-intake ratio, $(\mathrm{pCi} / \mathrm{L}) /(\mathrm{pCi} / \mathrm{d})$

$D-34$

D-34 3 Pu-239, plant/soil concentration ratio, dimensionless

$\mathrm{D}-343 \mathrm{Pu}-239$, beef/Iivestock-intake ratio, (pCi/kg)/(pCi/d)

$\mathrm{D}-34{ }^{3} \mathrm{Pu}-239$, milk/livestock-intake ratio, $(\mathrm{pCi} / \mathrm{L}) /(\mathrm{pCi} / \mathrm{d})$

$D-34{ }^{3}$

$\mathrm{D}-344^{3} \mathrm{Pu}-240$, plant/soil concentration ratio, dimensionless

$\mathrm{D}-343 \mathrm{Pu}-240$, beef/livestock-intake ratio, $(\mathrm{pCi} / \mathrm{kg}) /(\mathrm{pCi} / \mathrm{d})$

$\mathrm{D}-34{ }^{3} \mathrm{Pu}-240$, milk/livestock-intake ratio, $(\mathrm{pCi} / \mathrm{L}) /(\mathrm{pCi} / \mathrm{d})$

$\mathrm{D}-34^{3}$

D-34 3 Pu-241+D, plant/soil concentration ratio, dimensionless

$\mathrm{D}-343 \mathrm{pu}-241+\mathrm{D}$, beef/livestock-intake ratio, $(\mathrm{pCi} / \mathrm{kg}) /(\mathrm{pCi} / \mathrm{d})$

$\mathrm{D}-343 \mathrm{pu}-241+\mathrm{D}$, milk/livestock-intake ratio, $(\mathrm{pCi} / \mathrm{L}) /(\mathrm{pCi} / \mathrm{d})$

$\mathrm{D}-344^{3}$

D-34 $3 \mathrm{Ra}-226+\mathrm{D}$, plant/soil concentration ratio, dimensionless

$\mathrm{D}-343 \mathrm{Ra}-226+\mathrm{D}$, beef/livestock-intake ratio, (pCi/ $\mathrm{kg}) /(\mathrm{pCi} / \mathrm{d})$

D-34 $3 \mathrm{Ra}-226+\mathrm{D}$, milk/livestock-intake ratio, $(\mathrm{pCi} / \mathrm{L}) /(\mathrm{pCi} / \mathrm{d})$

$\mathrm{D}-344^{3}$

D-34 3 Ra-228+D, plant/soil concentration ratio, dimensionless D-34 3 Ra-228+D , beef/livestock-intake ratio, (pCi/kg)/(pCi/d) $\mathrm{D}-343 \mathrm{Ra}-228+\mathrm{D}$, milk/livestock-intake ratio, (pCi/L)/(pCi/d) $D-34 \quad 3$

\section{Current ${ }^{3}{ }^{3}$ Parameter}

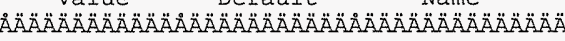

$32.500 \mathrm{E}-03^{3}{ }^{3} 2.500 \mathrm{E}-03^{3}{ }^{3} \operatorname{RTF}(1,1)$

$32.000 \mathrm{E}-05 \quad 3 \quad 2.000 \mathrm{E}-053 \operatorname{RTF}(1,2)$

32.000 E-05 32.000 E-05 $3 \operatorname{RTF}(1,3)$

1.000E-03 3 1.000E-03 3 RTF $(2,1)$

$35.000 \mathrm{E}-0535.000 \mathrm{E}-053 \operatorname{RTF}(2,2)$

32.000 E-06 32.000 E-06 $3 \operatorname{RTF}(2,3)$

34.000 E-02 $3 \quad 4.000 E-02 \quad 3 \operatorname{RTF}(3,1)$

$33.000 E-0233.000 E-023 \operatorname{RTF}(3,2)$

$38.000 \mathrm{E}-033^{3} 8.000 \mathrm{E}-033^{3} \operatorname{RTF}(3,3)$

3 . 3

$32.000 E-02 \quad 3 \quad 2.000 E-02 \quad 3 \operatorname{RTF}(4,1)$

$31.000 \mathrm{E}-0331.000 \mathrm{E}-033 \operatorname{RTF}(4,2)$

3 5.000E-06 3 5.000E-06 3 RTF $(4,3)$

33

$31.000 \mathrm{E}-0231.000 \mathrm{E}-023 \operatorname{RTF}(5,1)$

35.000 E-03 3 5.000 E-03 3 $\operatorname{RTF}(5,2)$

3 5.000E-06 3 5.000E-06 3 $\operatorname{RTF}(5,3)$

3

$31.000 \mathrm{E}-0231.000 \mathrm{E}-023 \operatorname{RTF}(6,1)$

$38.000 E-0438.000 E-043 \operatorname{RTF}(6,2)$

$33.000 E-04 \quad 3 \quad 3.000 E-043 \operatorname{RTF}(6,3)$

$3_{3} 3_{3}$

31.000 E-03 31.000 E-03 $3 \operatorname{RTF}(7,1)$

$31.000 E-04 \quad 3 \quad 1.000 E-043 \operatorname{RTF}(7,2)$

31.000 E-06 31.000 E-06 3 RTF $(7,3)$

${ }_{3} 1.000 \mathrm{E}-06_{3}{ }_{3} 1.000 \mathrm{E}-06_{3}$

$31.000 \mathrm{E}-03 \quad 3 \quad 1.000 \mathrm{E}-033 \operatorname{RTF}(8,1)$

$31.000 \mathrm{E}-0431.000 \mathrm{E}-043 \operatorname{RTF}(8,2)$

31.000 E-06 31.000 E-06 $3 \operatorname{RTF}(8,3)$

${ }_{3} 3_{3}$

$3 \quad 1.000 \mathrm{E}-03 \quad 3 \quad 1.000 \mathrm{E}-03 \quad 3 \operatorname{RTF}(9,1)$

$31.000 \mathrm{E}-04 \quad 3 \quad 1.000 \mathrm{E}-043 \operatorname{RTF}(9,2)$

3 1.000E-06 $331.000 \mathrm{E}-06^{3} \operatorname{RTE}(9,3)$

${ }_{3}{ }_{3}{ }_{3}$

$31.000 \mathrm{E}-03^{3} 1.000 \mathrm{E}-03^{3} \operatorname{RTF}^{3}(10,1)$

$31.000 \mathrm{E}-04 \quad 3 \quad 1.000 \mathrm{E}-04 \quad 3 \operatorname{RTF}(10,2)$

31.000 E-06 31.000 E-06 $3 \operatorname{RTF}(10,3)$

${ }_{3}{ }_{3} 1.0008-06{ }_{3}$

$34.000 \mathrm{E}-0234.000 \mathrm{E}-023 \operatorname{RTF}(12,1)$

$31.000 E-03 \quad 3 \quad 1.000 E-03 \quad 3 \operatorname{RTF}(12,2)$

${ }^{3} 1.000 \mathrm{E}-033^{3} 1.000 \mathrm{E}-033 \operatorname{RTF}(12,3)$

$34.000 \mathrm{E}-02 \quad 3 \quad 4.000 \mathrm{E}-02 \quad 3 \quad \operatorname{RTF}(13,1)$

$31.000 \mathrm{E}-03 \quad 3 \quad 1.000 \mathrm{E}-03 \quad 3 \quad \operatorname{RTF}(13,2)$

${ }_{3} 1.000 \mathrm{E}-033_{3} 1.000 \mathrm{E}-033_{3} \operatorname{RTF}(13,3)$ 


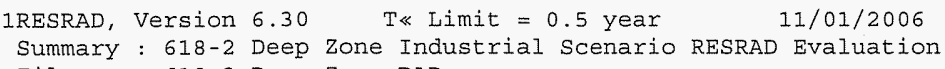

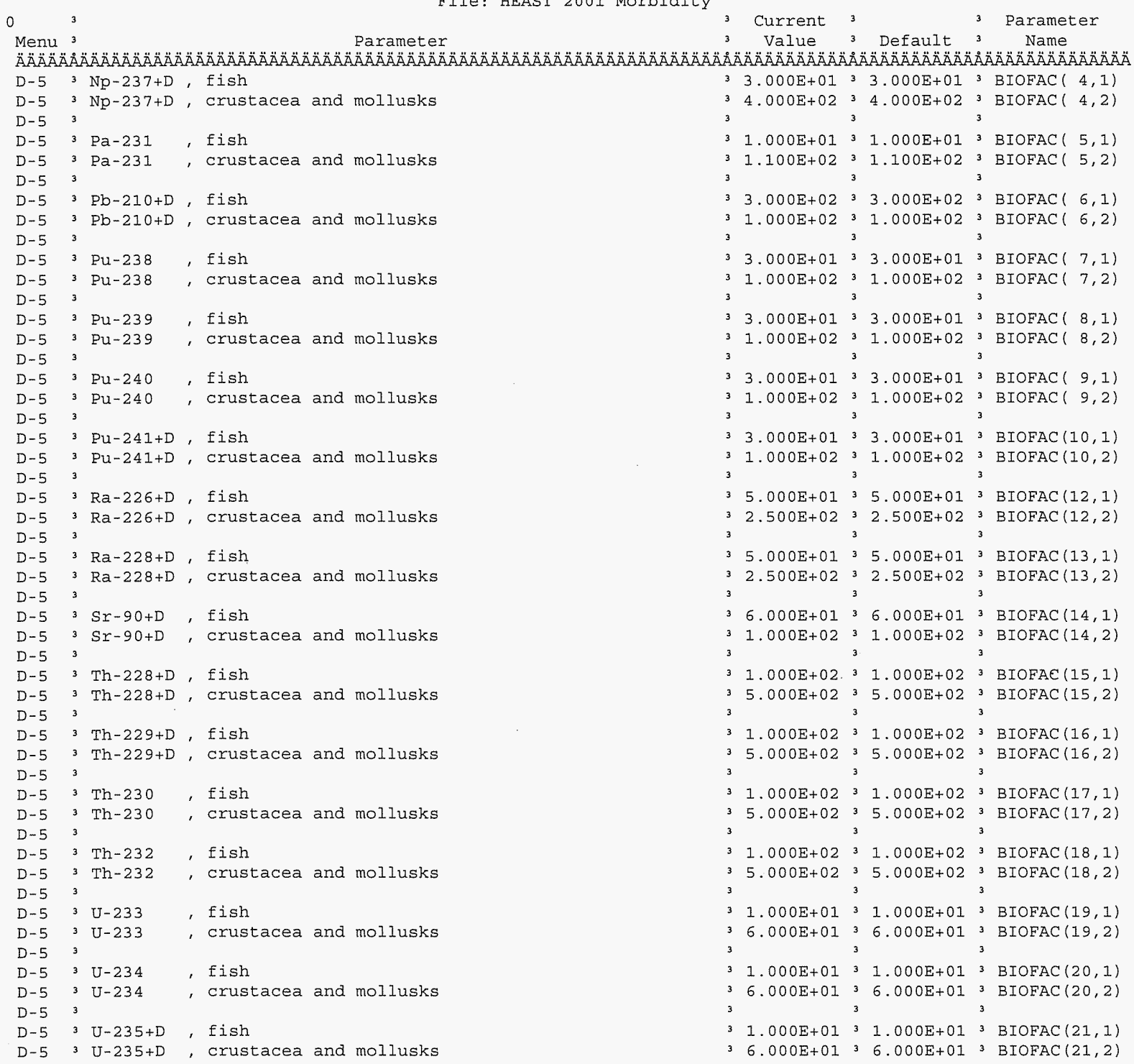




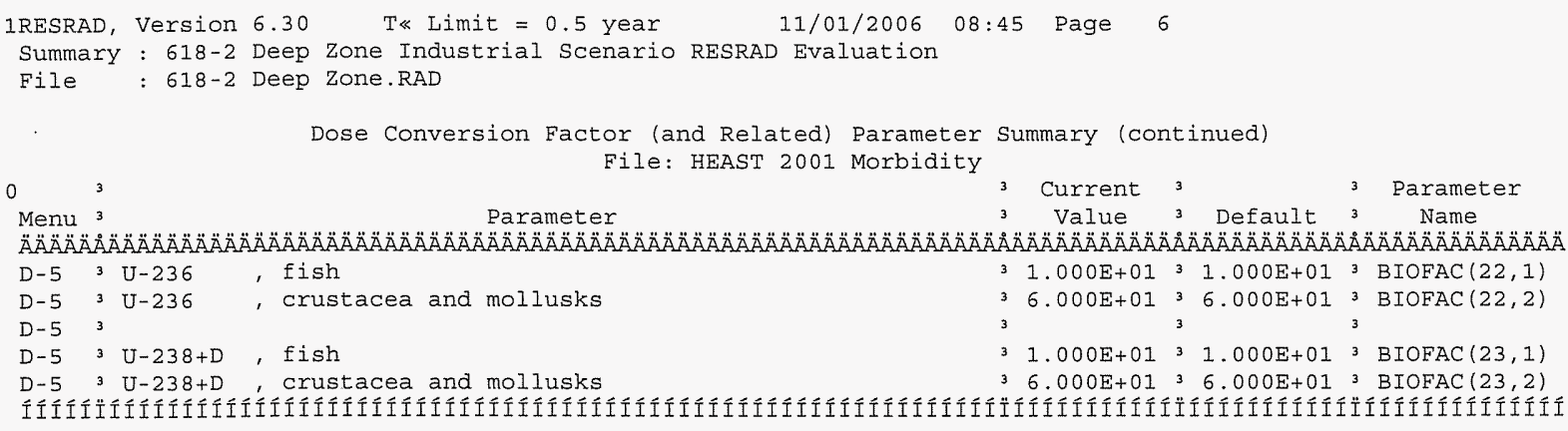


1RESRAD, Version $6.30 \quad$ T\& Limit $=0.5$ year $11 / 01 / 2006 \quad 08: 45$ Page 7 Summary : 618-2 Deep Zone Industrial Scenario RESRAD Evaluation

File : 618-2 Deep Zone.RAD

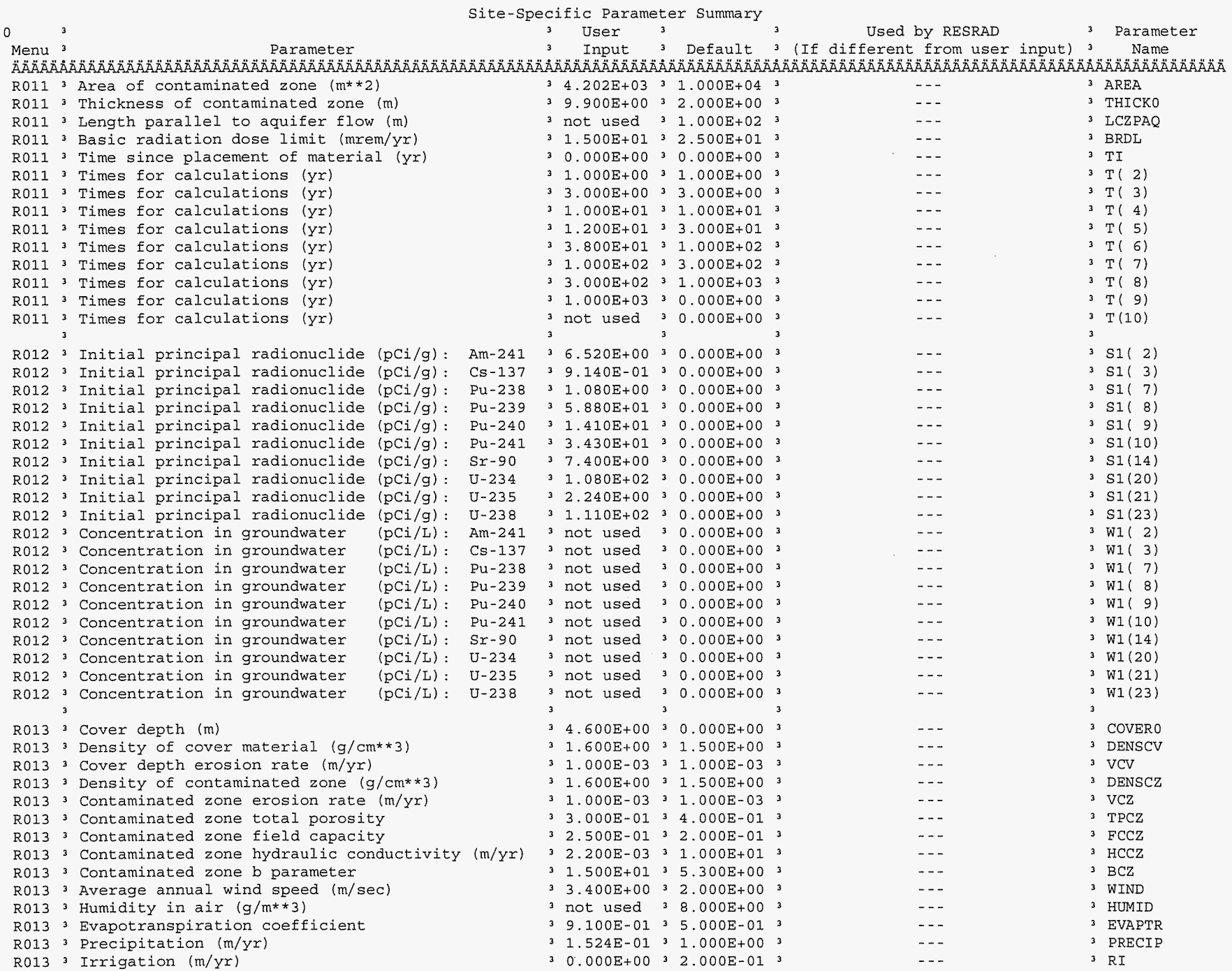


1RESRAD, Version $6.30 \quad T \ll$ Limit $=0.5$ year $\quad 11 / 01 / 2006 \quad 08: 45$ Page 8

Summary : 618-2 Deep Zone Industrial Scenario RESRAD Evaluation

File : 618-2 Deep Zone.RAD

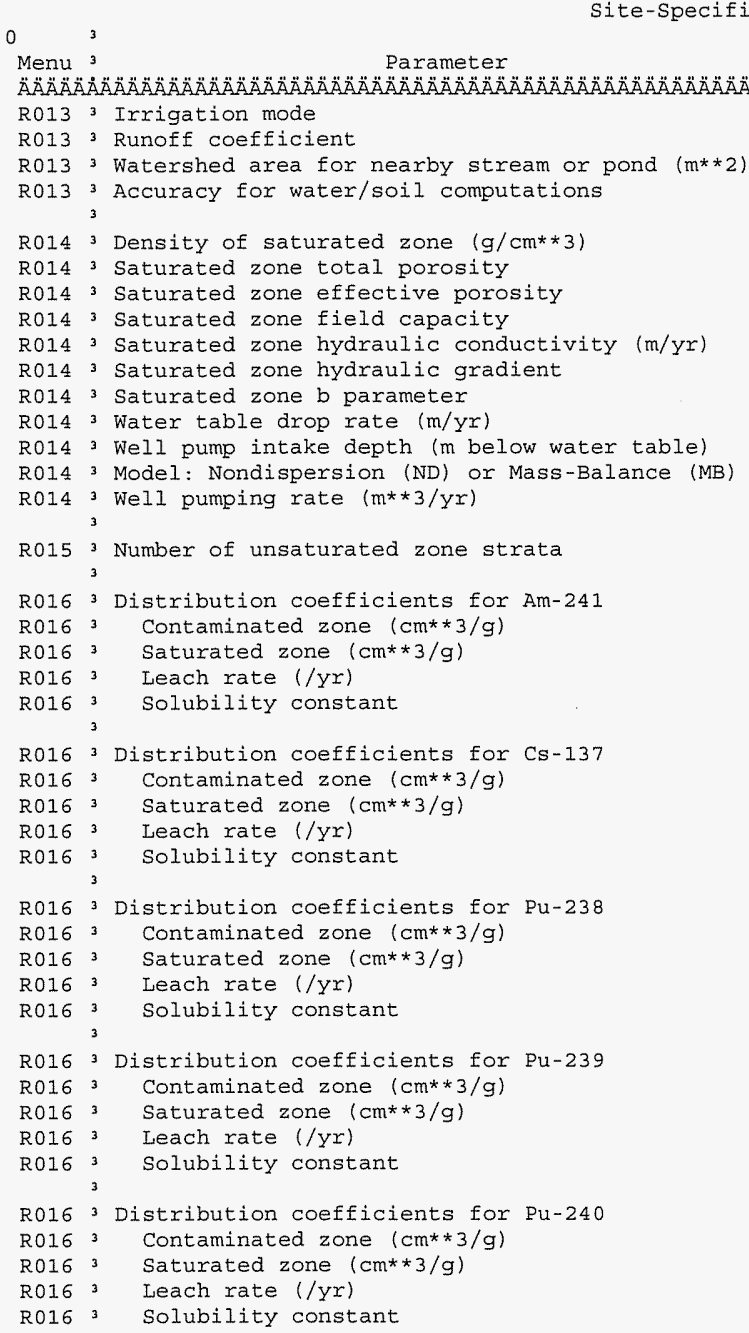

Parameter Summary (continued)

3 User ${ }_{3} \quad{ }_{3}$ Used by RESRAD Parameter

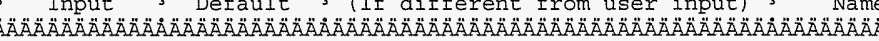

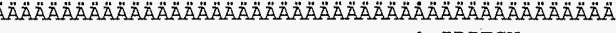
( - - 3 IDITCH $2.000 \mathrm{E}-0132.000 \mathrm{E}-0133$ RUNOFF

3 not used $31.000 \mathrm{E}+06^{3} \quad 3$ WAREA ${ }^{3}$ not used ${ }^{3} 1.000 E_{-03}{ }^{3}$ Romberg failures occurred ${ }_{3}$ EPS 3 not used $31.500 \mathrm{E}+00$ 3 not used 34.000 E-013

not used 3 2.000E-01

mot used $32.0000-01$

$-01$

3 not used $31.000 \mathrm{E}+02$

3 not used $32.000 \mathrm{E}-02$

3 not used $35.300 \mathrm{E}+00$

3 not used $31.000 \mathrm{E}-03$

3 not used 3 1.000E+01

3 not used 3 ND

3 not used $32.500 E+02$

not used. 3

$2.000 E+0232.000 E+01$

not used $32.000 E+01$

$0.000 E+0030.000 E+00$

$0.000 \mathrm{E}+0030.000 \mathrm{E}+00$

$5.000 E+01^{3} 1.000 E+03$

not used $31.000 \mathrm{E}+03^{3}$

$0.000 E+00 \quad 3 \quad 0.000 E+00^{3}$

$30.000 \mathrm{E}+00^{3} 0.000 \mathrm{E}+00^{3}$

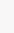

$2.000 \mathrm{E}+0232.000 \mathrm{E}+03$

not used $32.000 E+03$

$0.000 \mathrm{E}+00^{3} \quad 0.000 \mathrm{E}+00^{3}$

$0.000 E+0030.000 E+00$

$2.000 E+0232.000 E+03$

3 not used $32.000 E+03$

$0.000 E+0030.000 E+00$

$0.000 \mathrm{E}+00 \quad 3 \quad 0.000 \mathrm{E}+00$

$2.000 \mathrm{E}+0232.000 \mathrm{E}+03$

not used $32.000 \mathrm{E}+03=$

$0.000 \mathrm{E}+00 \quad 3 \quad 0.000 \mathrm{E}+00$

$30.000 E+0030.000 E+00^{3}$

\begin{tabular}{|c|c|c|c|}
\hline$\cdots$ & \multicolumn{3}{|c|}{3 DENSAQ } \\
\hline$\cdots$ & 3 & \multicolumn{2}{|l|}{ TPSZ } \\
\hline$\cdots$ & 3 & \multicolumn{2}{|l|}{ EPSZ } \\
\hline$-\cdots$ & 3 & \multicolumn{2}{|l|}{ FCSZ } \\
\hline$\ldots$ & 3 & \multicolumn{2}{|l|}{$\mathrm{HCSZ}$} \\
\hline$\cdots$ & 3 & \multicolumn{2}{|l|}{ HGWT } \\
\hline$\ldots$ & 3 & \multicolumn{2}{|l|}{$\mathrm{BSZ}$} \\
\hline$\ldots$ & 3 & \multicolumn{2}{|c|}{ VWT } \\
\hline-- & 3 & \multicolumn{2}{|l|}{ DWIBWT } \\
\hline-- & 3 & \multicolumn{2}{|l|}{ MODEL } \\
\hline--- & $\begin{array}{l}3 \\
3\end{array}$ & \multicolumn{2}{|l|}{ UW } \\
\hline.- & 3 & \multirow{2}{*}{\multicolumn{2}{|c|}{ NS }} \\
\hline & 3 & & \\
\hline & \multicolumn{3}{|l|}{3} \\
\hline.-- & 3 & DCNUCC & 2) \\
\hline$-\cdots$ & 3 & DCNUCS ( & 2) \\
\hline $3.460 \mathrm{E}-06$ & 3 & ALEACH ( & 2) \\
\hline \multirow[t]{3}{*}{ not used } & 3 & SOLUBKI & 2) \\
\hline & \multirow{2}{*}{\multicolumn{3}{|c|}{3}} \\
\hline & & & \\
\hline$-\ldots$ & 3 & \multicolumn{2}{|c|}{ DCNUCC ( 3) } \\
\hline$-\cdots$ & 3 & \multicolumn{2}{|c|}{ DCNUCS ( 3 ) } \\
\hline 1. $380 \mathrm{E}-05$ & 3 & \multicolumn{2}{|c|}{ ALEACH ( 3) } \\
\hline not used & 3 & \multirow{2}{*}{\multicolumn{2}{|c|}{ SOLUBK ( 3) }} \\
\hline & 3 & & \\
\hline & \multicolumn{3}{|l|}{3} \\
\hline$-\ldots$ & 3 & \multicolumn{2}{|c|}{ DCNUCC ( 7) } \\
\hline-- & 3 & \multicolumn{2}{|c|}{ DCNUCS ( 7) } \\
\hline $3.460 E-06$ & 3 & \multicolumn{2}{|c|}{ ALEACH ( 7) } \\
\hline not used & 3 & \multirow{2}{*}{\multicolumn{2}{|c|}{ SOLUBK ( 7) }} \\
\hline & 3 & & \\
\hline & 3 & & \\
\hline$\ldots$ & 3 & DCNUCC ( & 8) \\
\hline-- & 3 & DCNUCS ( & 8) \\
\hline $3.460 \mathrm{E}-06$ & 3 & ALEACH（ & 8) \\
\hline not used & 3 & SOLUBKI & 8) \\
\hline & 3 & & \\
\hline & 3 & & \\
\hline$\cdots$ & 3 & DCNUCC & 9) \\
\hline-- & 3 & DCNUCS ( & 9) \\
\hline $3.460 \mathrm{E}-06$ & 3 & ALEACH（ & 9) \\
\hline not used & 3 & SOLUBK! & 9) \\
\hline
\end{tabular}




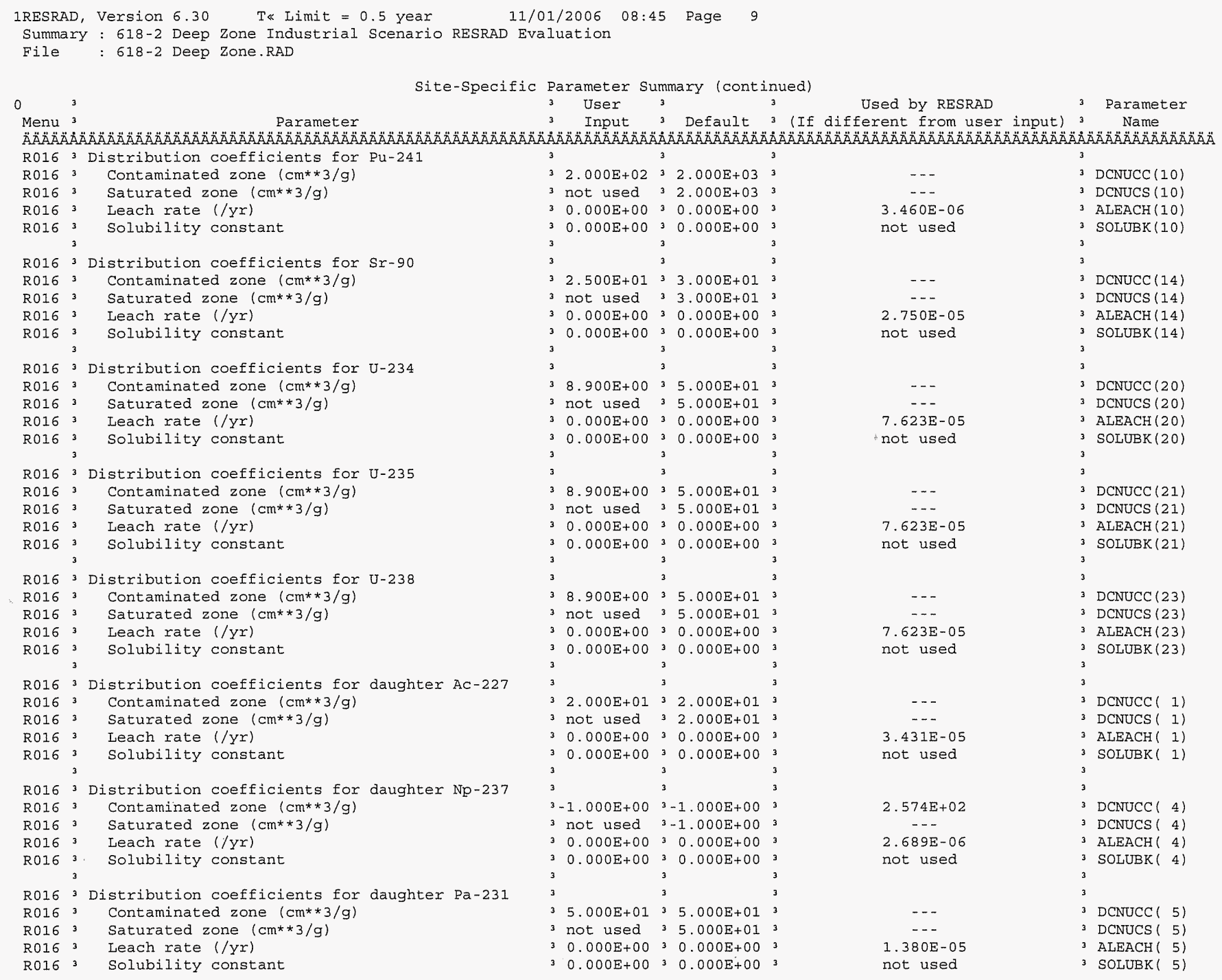


IRESRAD, Version $6.30 \quad$ T\& Limit $=0.5$ year $11 / 01 / 2006 \quad 08: 45$ Page 10

Summary : 618-2 Deep Zone Industrial Scenario RESRAD Evaluation

File : 618-2 Deep Zone.RAD

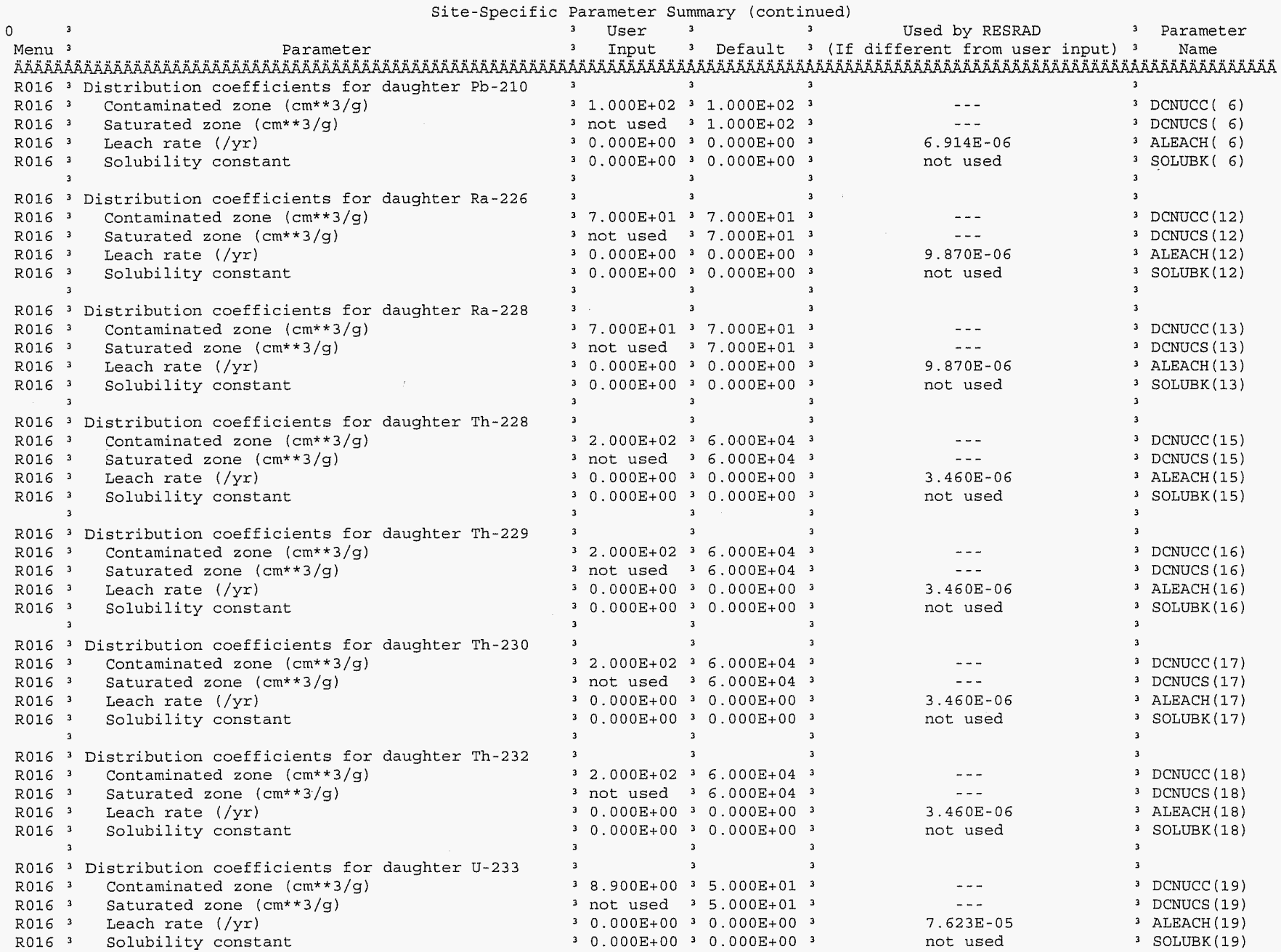




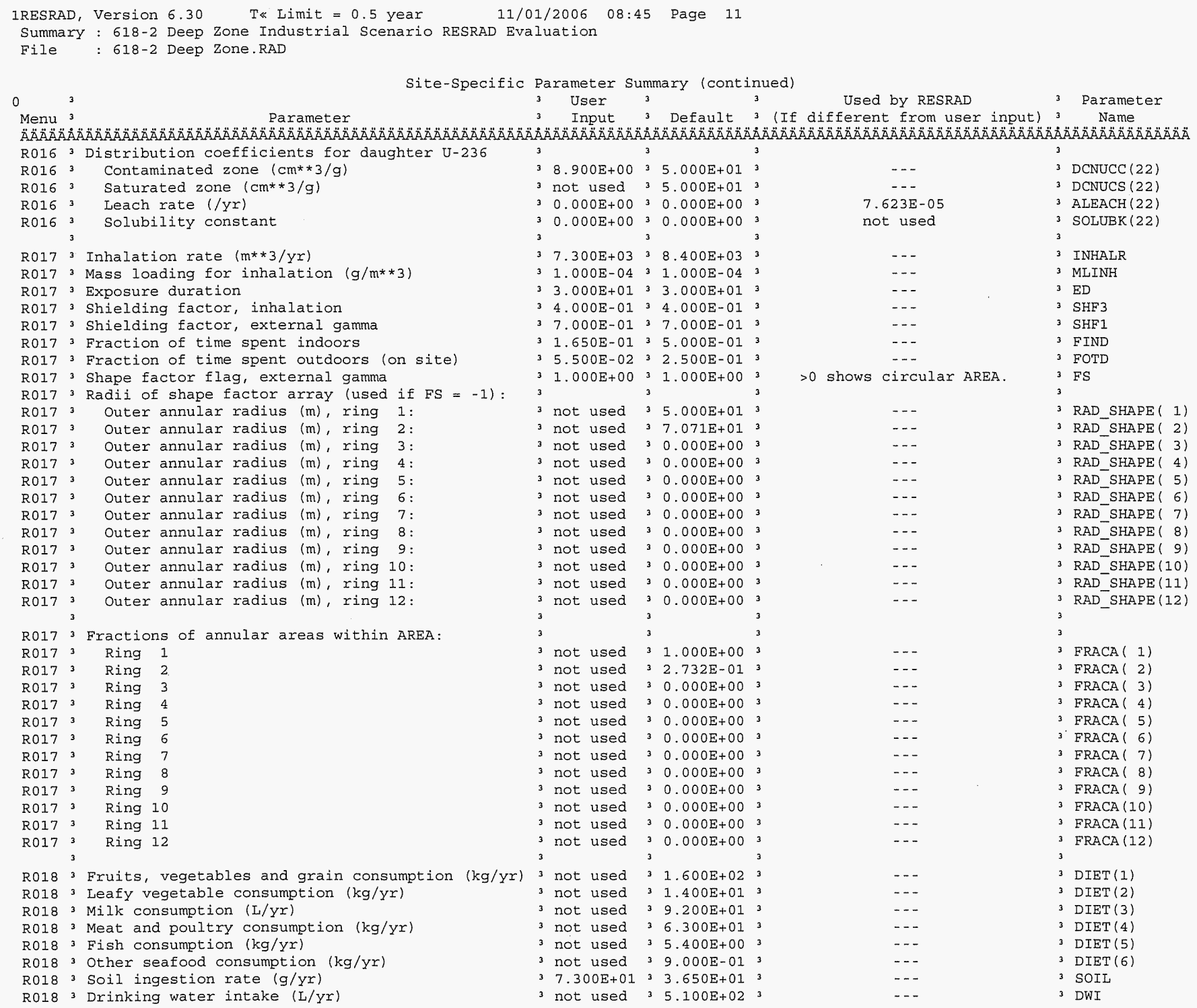


1RESRAD, Version $6.30 \quad$ T\& Limit $=0.5$ year $\quad 11 / 01 / 2006 \quad 08: 45$ Page 12
Summary : $618-2$ Deep zone Industrial Scenario RESRAD Evaluation

File : 618-2 Deep Zone.RAD

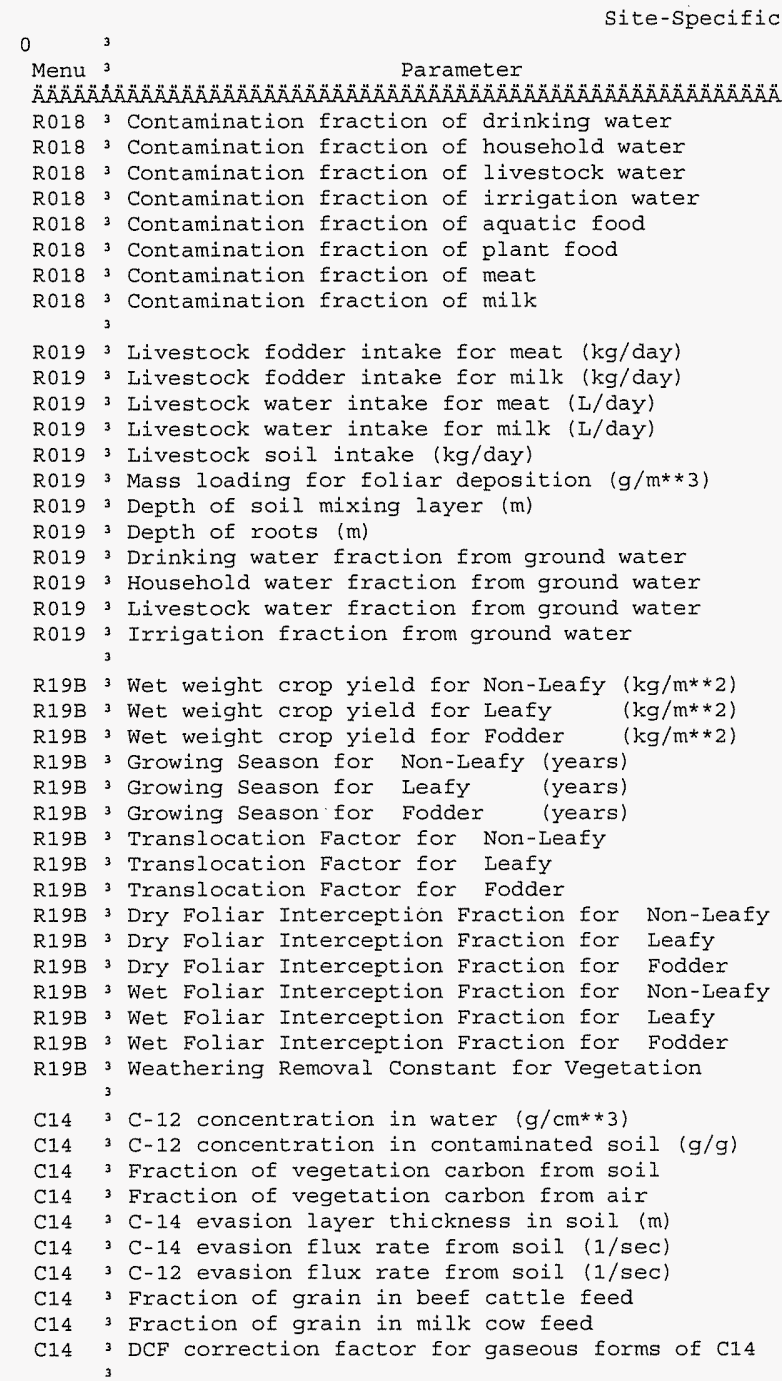

Parameter Summary (continued)

${ }_{3}$ User ${ }_{3}^{3}$ Input ${ }_{3}$ Default ${ }_{3}^{3}$ (If different Used by RESRAD

Used bY RESRAD 3 Parameter

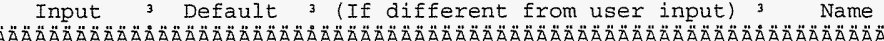

3 not used $31.000 \mathrm{E}+00^{3}$

3 not used $31.000 E+003$

3 not used $31.000 \mathrm{E}+00^{3}$

not used $31.000 \mathrm{E}+00$

3 not used 3 5.000E-01

3 not used $3-1$

3 not used $3-1$

3 not used $3-1$

3 not used $36.800 E+01$

3 not used $35.500 E+01$

3 not used $35.000 E+013$

3 not used $31.600 \mathrm{E}+02$

3 not used 3 5.000E-01 3

not used 31.000 E-04

31.500 E-01 31.500 E-01

3 not used $39.000 \mathrm{E}-01$

3 not used

3 not used $31.000 E+00_{3}$

3 not used $31.000 \mathrm{E}+00^{3}$

3 not used $31.000 \mathrm{E}+00$

3 not used $37.000 \mathrm{E}-01$

3 not used $31.500 \mathrm{E}+00^{3}$

3 not used $31.100 \mathrm{E}+00$

3 not used $31.700 \mathrm{E}-01^{3}$

3 not used 32.500 E-01

3 not used 38.000 E-02

3 not used $31.000 \mathrm{E}-01$

not used

3 not used $31.000 \mathrm{E}+00^{3}$

3 not used 32.500 E-01

3 not used $32.500 \mathrm{E}-01$

3 not used 32 500E-01

not used $32.500 \mathrm{E}-01$

3 not used $32.500 \mathrm{E}-01$

3 not used $32.500 E-01$

3 not used $32.000 E+01$

not used 32.000 E-05

3 not used 33.000 E-02

not used $32.000 \mathrm{E}-02$

not used $39.800 \mathrm{E}-01$

not used 3 3.000E-01

3 not used 37.000 E-07

not used $31.000 \mathrm{E}-10$

3 not used $38.000 \mathrm{E}-01$

3 not used $32.000 \mathrm{E}-01$

${ }_{3}$ not used ${ }_{3} 8.894 \mathrm{E}+011_{3}$

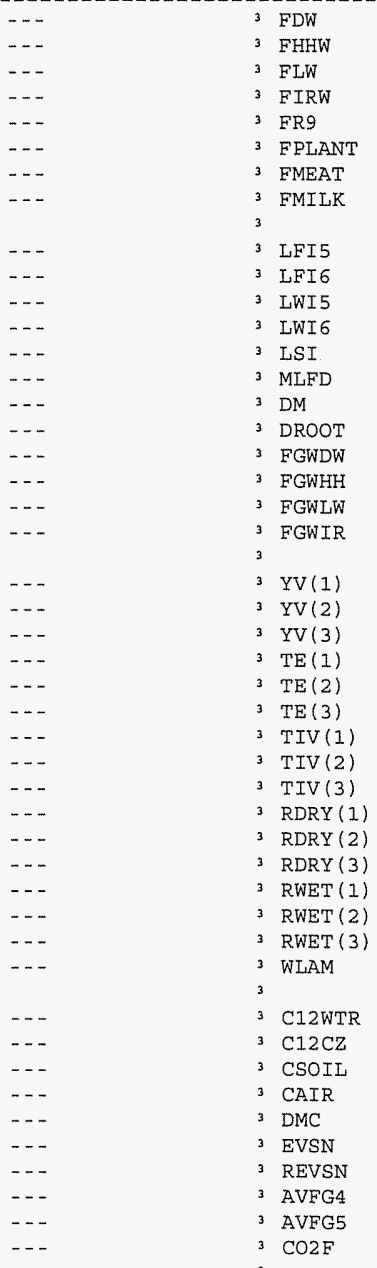




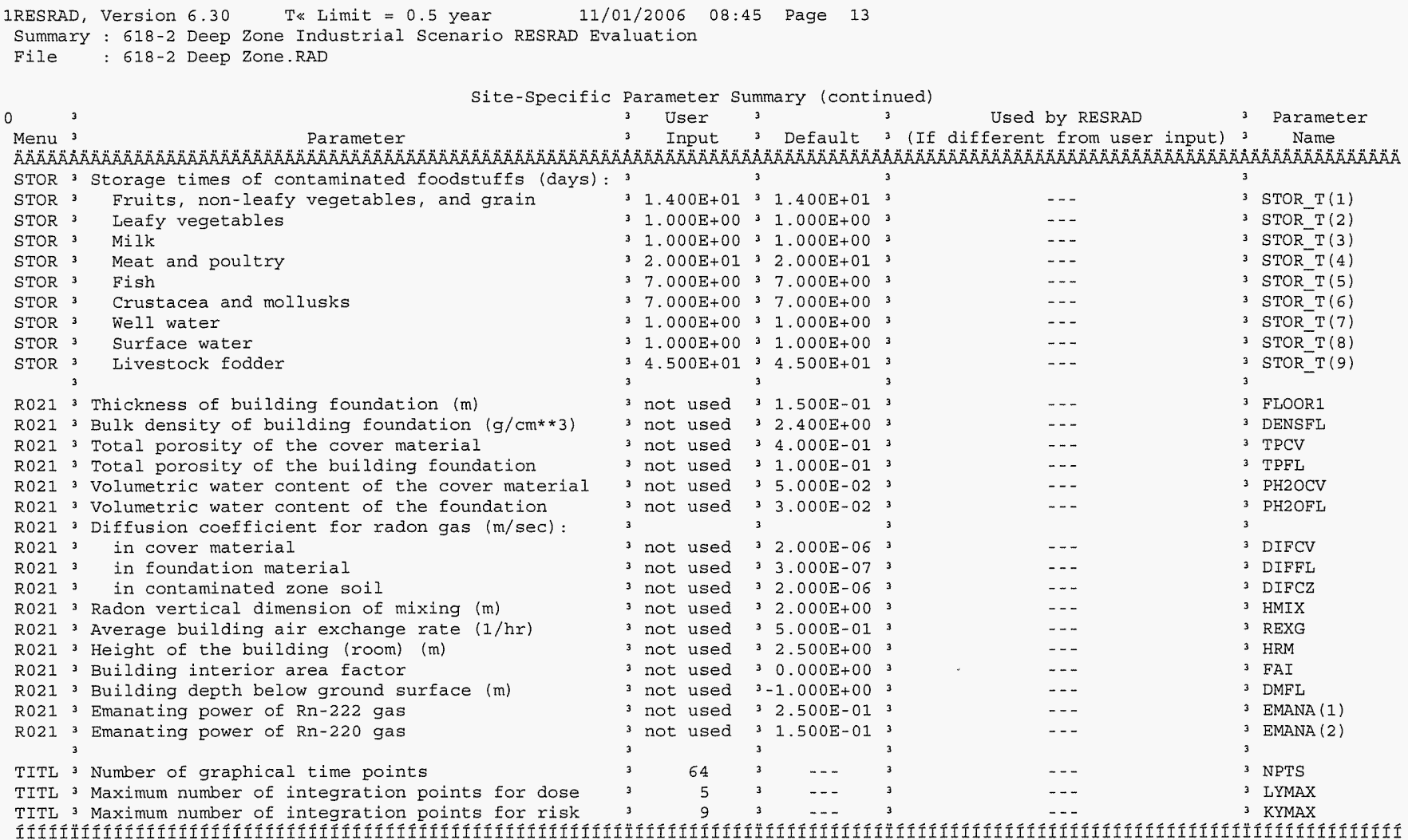

Summary of Pathway Selections

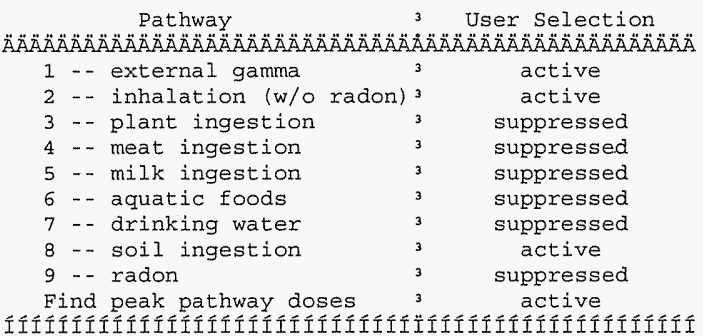




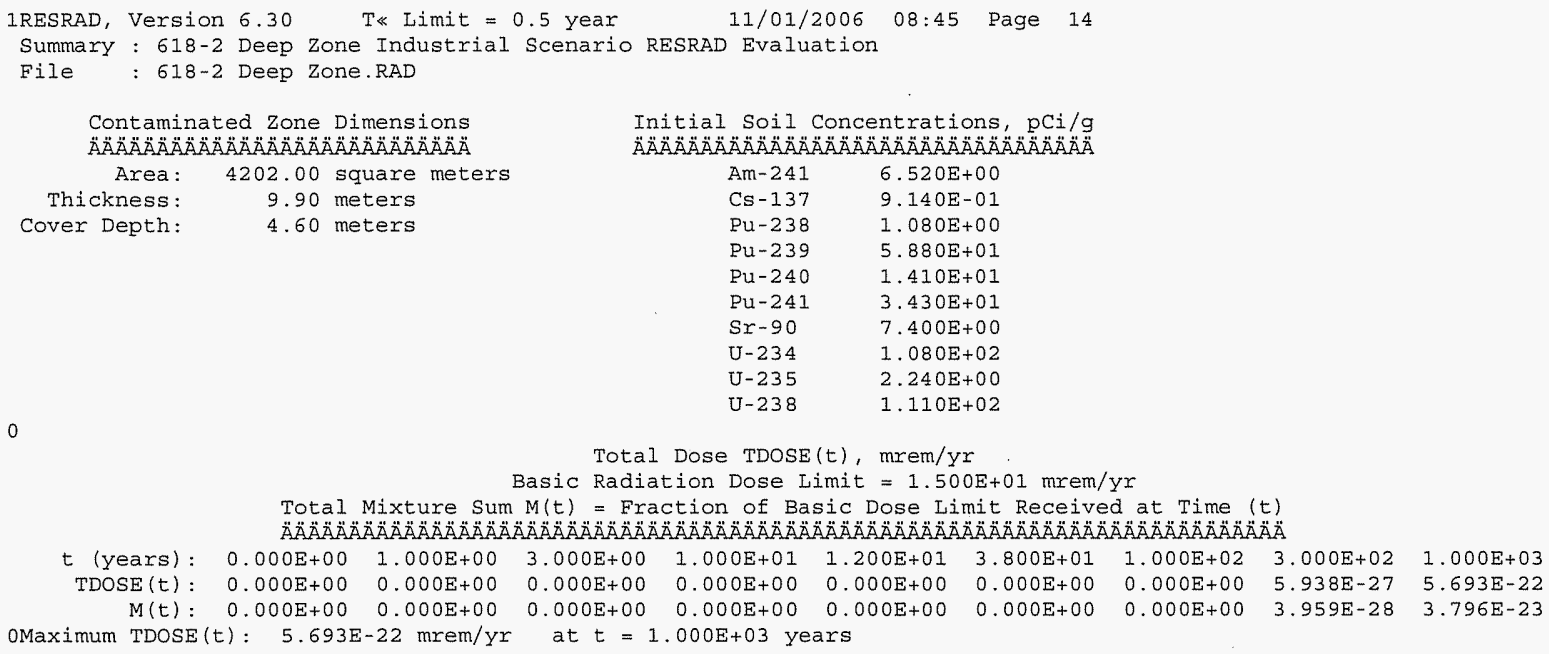


File : 618-2 staging Pile.RAD

Dose Conversion Factor (and Related) Parameter Summary File: HEAST 2001 Morbidity

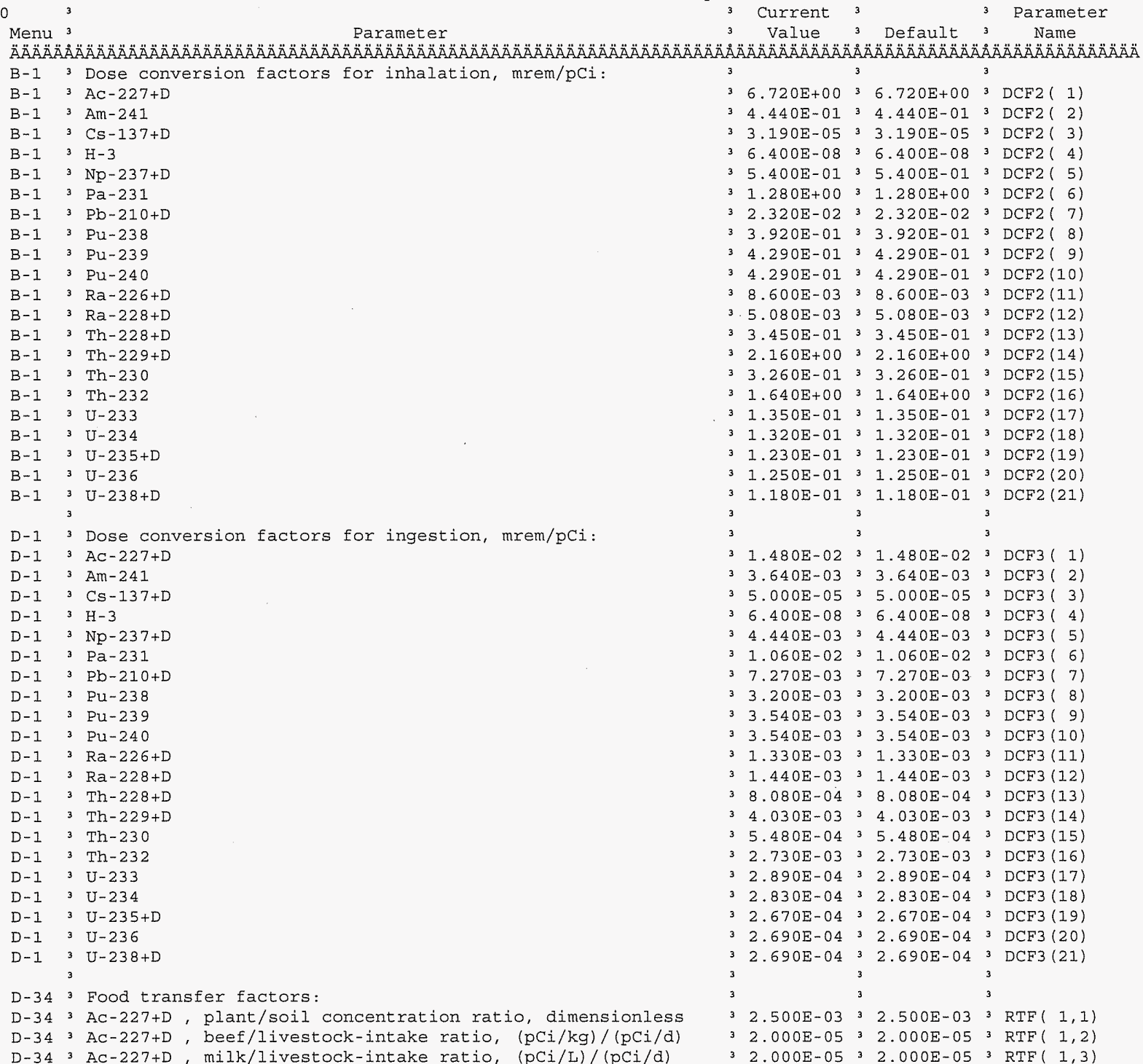


1RESRAD, Version $6.30 \quad T \ll$ Limit $=0.5$ year $\quad 11 / 01 / 2006 \quad 08: 58$ Page
Summary : $618-2$ Staging Pile and Decontamination Pad Area RESRAD Evaluation

File : 618-2 staging Pile.RAD

Dose Conversion Factor (and Related) Parameter Summary (continued)

\begin{tabular}{|c|c|c|c|c|c|c|c|c|}
\hline 0 & 3 & & 3 & Current & 3 & & 3 & Parameter \\
\hline Menu & 3 & meter & 3 & Value & 3 & Default & 3 & Name \\
\hline$\ddot{A} \ddot{A} \ddot{A}$ & & 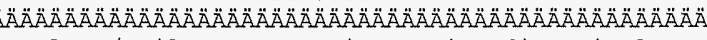 & & & & & & \\
\hline$D-34$ & $3 \mathrm{Am}-241$ & , plant/soil concentration ratio, dimensionless & 3 & $1.000 E-03$ & 3 & $1.000 \mathrm{E}-03$ & 3 & $\operatorname{RTF}(2,1)$ \\
\hline$D-34$ & $3 \mathrm{Am}-241$ & beef/livestock-intake ratio, $(\mathrm{pci} / \mathrm{kg}) /(\mathrm{pCi} / \mathrm{d})$ & 3 & $5.000 \mathrm{E}-05$ & 3 & $5.000 \mathrm{E}-05$ & 3 & $\operatorname{RTF}(2,2)$ \\
\hline$D-34$ & 3 Am-241 & , milk/livestock-intake ratio, $(\mathrm{pCi} / \mathrm{L}) /(\mathrm{pCi} / \mathrm{d})$ & 3 & $2.000 \mathrm{E}-06$ & 3 & $2.000 E-06$ & 3 & $\operatorname{RTF}(2,3)$ \\
\hline$D-34$ & 3 & & 3 & & 3 & & 3 & \\
\hline$D-34$ & $3 \mathrm{Cs}-137+\mathrm{D}$ & plant/soil concentration ratio, dimensionless & 3 & $4.000 E-02$ & 3 & $4.000 \mathrm{E}-02$ & 3 & $\operatorname{RTE}(3,1)$ \\
\hline$D-34$ & $3 \mathrm{Cs}-137+\mathrm{D}$ & , beef/livestock-intake ratio, $(\mathrm{pCi} / \mathrm{kg}) /(\mathrm{pCi} / \mathrm{d})$ & 3 & $3.000 E-02$ & 3 & $3.000 E-02$ & 3 & $\operatorname{RTF}(3,2)$ \\
\hline$D-34$ & ${ }^{3} \mathrm{Cs}-137+\mathrm{D}$ & , milk/livestock-intake ratio, $(\mathrm{pCi} / \mathrm{L}) /(\mathrm{pCi} / \mathrm{d})$ & 3 & $8.000 E-03$ & 3 & $8.000 \mathrm{E}-03$ & 3 & $\operatorname{RTF}(3,3)$ \\
\hline$D-34$ & 3 & & 3 & & 3 & & 3 & \\
\hline$D-34$ & $3 \mathrm{H}-3$ & , plant/soil concentration ratio, dimensionless & 3 & $4.800 \mathrm{E}+00$ & 3 & $4.800 \mathrm{E}+00$ & 3 & $\operatorname{RTF}(4,1)$ \\
\hline$D-34$ & $3 \mathrm{H}-3$ & , beef/livestock-intake ratio, $(\mathrm{pCi} / \mathrm{kg}) /(\mathrm{pCi} / \mathrm{d})$ & 3 & $1.200 \mathrm{E}-02$ & 3 & $1.200 E-02$ & 3 & $\operatorname{RTF}(4,2)$ \\
\hline$D-34$ & $3 \mathrm{H}-3$ & , milk/Iivestock-intake ratio, $(\mathrm{pCi} / \mathrm{L}) /(\mathrm{pCi} / \mathrm{d})$ & 3 & $1.000 \mathrm{E}-02$ & 3 & $1.000 E-02$ & 3 & $\operatorname{RTF}(4,3)$ \\
\hline$D-34$ & 3 & & 3 & & 3 & & 3 & \\
\hline$D-34$ & $3 \mathrm{~Np}-237+\mathrm{D}$ & , plant/soil concentration ratio, dimensior & 3 & $2.000 \mathrm{E}-02$ & 3 & $2.000 \mathrm{E}-02$ & 3 & $\operatorname{RTF}(5,1)$ \\
\hline$D-34$ & $3 \mathrm{~Np}-237+\mathrm{D}$ & , beef/livestock-intake ratio, $(\mathrm{pCi} / \mathrm{kg}) /(\mathrm{pCi} / \mathrm{d})$ & 3 & $1.000 E-03$ & 3 & $1.000 \mathrm{E}-03$ & 3 & $\operatorname{RTF}(5,2)$ \\
\hline$D-34$ & $3 \mathrm{~Np}-237+\mathrm{D}$ & , milk/livestock-intake ratio, $(\mathrm{pCi} / \mathrm{L}) /(\mathrm{pCi} / \mathrm{d})$ & 3 & $5.000 \mathrm{E}-06$ & 3 & $5.000 \mathrm{E}-06$ & 3 & $\operatorname{RTF}(5,3)$ \\
\hline$D-34$ & 3 & & 3 & & 3 & & 3 & \\
\hline$D-34$ & $3 \mathrm{~Pa}-231$ & , plant/soil concentration ratio, dime & 3 & $1.000 \mathrm{E}-02$ & 3 & $1.000 E-02$ & 3 & $\operatorname{RTF}(6,1)$ \\
\hline$D-34$ & $3 \mathrm{~Pa}-231$ & , beef/livestock-intake ratio, $(\mathrm{pCi} / \mathrm{kg}) /(\mathrm{pCi} / \mathrm{d})$ & 3 & $5.000 E-03$ & 3 & $5.000 \mathrm{E}-03$ & 3 & $\operatorname{RTE}(6,2)$ \\
\hline$D-34$ & $3 \mathrm{~Pa}-231$ & , milk/livestock-intake ratio, $(\mathrm{pCi} / \mathrm{L}) /(\mathrm{pCi} / \mathrm{d})$ & 3 & $5.000 E-06$ & 3 & $5.000 \mathrm{E}-06$ & 3 & $\operatorname{RTF}(6,3)$ \\
\hline$D-34$ & 3 & & 3 & & 3 & & 3 & \\
\hline$D-34$ & $3 \mathrm{~Pb}-210+\mathrm{D}$ & , plant/soil concentration ratio, dimensionless & 3 & 1. $000 \mathrm{E}-02$ & 3 & $1.000 \mathrm{E}-02$ & 3 & $\operatorname{RTF}(7,1)$ \\
\hline$D-34$ & $3 \mathrm{~Pb}-210+\mathrm{D}$ & , beef/livestock-intake ratio, $(\mathrm{pCi} / \mathrm{kg}) /(\mathrm{pCi} / \mathrm{d})$ & 3 & $8.000 \mathrm{E}-04$ & 3 & $8.000 E-04$ & 3 & $\operatorname{RTF}(7,2)$ \\
\hline$D-34$ & $3 \mathrm{~Pb}-210+\mathrm{D}$ & , $\mathrm{milk} /$ livestock-intake ratio, $(\mathrm{pCi} / \mathrm{L}) /(\mathrm{pCi} / \mathrm{d})$ & 3 & $3.000 E-04$ & 3 & $3.000 \mathrm{E}-04$ & 3 & $\operatorname{RTF}(7,3)$ \\
\hline$D-34$ & 3 & & 3 & & 3 & & 3 & \\
\hline$D-34$ & 3 Pu-238 & , plant/soil concentration ratio, dime & 3 & $1.000 \mathrm{E}-03$ & 3 & E-03 & 3 & $\operatorname{RTE}(8,1)$ \\
\hline$D-34$ & $3 \mathrm{Pu}-238$ & , beef/livestock-intake ratio, (pCi/ks & 3 & $0 E-04$ & 3 & $E-04$ & 3 & $\operatorname{RTE}(8,2)$ \\
\hline$D-34$ & $3 \mathrm{Pu}-238$ & , milk/livestock-intake ratio, $(\mathrm{pCi} / \mathrm{L}) /(\mathrm{pCi} / \mathrm{d})$ & 3 & $1.000 \mathrm{E}-06$ & 3 & $1.000 E-06$ & 3 & $\operatorname{RTF}(8,3)$ \\
\hline$D-34$ & 3 & & 3 & & 3 & & 3 & \\
\hline$D-34$ & s Pu-239 & oil concentration ratio, dim & 3 & $1.000 \mathrm{E}-03$ & 3 & $1.000 E-03$ & 3 & $\operatorname{RTF}(9,1)$ \\
\hline$D-34$ & 3 Pu-239 & , beef/Iivestock-intake ratio, $(\mathrm{pCi} / \mathrm{k}$ & 3 & OE-04 & 3 & $\mathrm{E}-04$ & 3 & $\operatorname{RTF}(9,2)$ \\
\hline$D-34$ & $3 \mathrm{Pu}-239$ & milk/livestock-intake ratio, $(\mathrm{pCi} / \mathrm{L}) /(\mathrm{pCi} / \mathrm{d})$ & 3 & $1.000 \mathrm{E}-06$ & 3 & $1.000 \mathrm{E}-06$ & 3 & $\operatorname{RTF}(9,3)$ \\
\hline$D-34$ & 3 & & 3 & & 3 & & 3 & \\
\hline$D-34$ & $3 \mathrm{Pu}-240$ & oil concentration ratio, dim & 3 & $1.000 \mathrm{E}-03$ & 3 & $1.000 \mathrm{E}-03$ & 3 & $\operatorname{RTF}(10,1)$ \\
\hline $\mathrm{D}-34$ & $3 \mathrm{Pu}-240$ & , beef/livestock-intake ratio, $(\mathrm{pCi} / \mathrm{kg}) /(\mathrm{pCi} / \mathrm{d})$ & 3 & $1.000 \mathrm{E}-04$ & 3 & $1.000 \mathrm{E}-04$ & 3 & $\operatorname{RTF}(10,2)$ \\
\hline$D-34$ & ${ }^{3} \mathrm{Pu}-240$ & , $\mathrm{milk} /$ livestock-intake ratio, $(\mathrm{pCi} / \mathrm{L}) /(\mathrm{pCi} / \mathrm{d})$ & 3 & $1.000 E-06$ & 3 & $1.000 \mathrm{E}-06$ & 3 & $\operatorname{RTF}(10,3)$ \\
\hline$D-34$ & 3 & & 3 & & 3 & & 3 & \\
\hline$D-34$ & ${ }^{3} \mathrm{Ra}-226+\mathrm{D}$ & , plant/soil concentration ratio, dim & 3 & $4.000 \mathrm{E}-02$ & 3 & $4.000 \mathrm{E}-02$ & 3 & $\operatorname{RTF}(11,1)$ \\
\hline$D-34$ & ${ }^{3} \mathrm{Ra}-226+\mathrm{D}$ & , beef/livestock-intake ratio, (pCi/kg)/(pCi/d) & 3 & $1.000 \mathrm{E}-03$ & 3 & $1.000 \mathrm{E}-03$ & 3 & $\operatorname{RTF}(11,2)$ \\
\hline$D-34$ & $3 \mathrm{R} a-226+\mathrm{D}$ & , milk/livestock-intake ratio, $(\mathrm{pCi} / \mathrm{L}) /(\mathrm{pCi} / \mathrm{d})$ & 3 & $1.000 \mathrm{E}-03$ & 3 & $1.000 \mathrm{E}-03$ & 3 & $\operatorname{RTF}(11,3)$ \\
\hline$D-34$ & 3 & & 3 & & 3 & & 3 & \\
\hline$D-34$ & $3 \mathrm{Ra}-228+\mathrm{D}$ & , plant/soil concentration ratio, dime & 3 & 4. $000 \mathrm{E}-02$ & 3 & $4.000 \mathrm{E}-02$ & 3 & $\operatorname{RTF}(12,1)$ \\
\hline$D-34$ & $3 \mathrm{Ra}-228+\mathrm{D}$ & , beef/livestock-intake ratio, (pCi/kg)/(pCi/d) & 3 & $1.000 \mathrm{E}-03$ & 3 & $1.000 \mathrm{E}-03$ & 3 & $\operatorname{RTF}(12,2)$ \\
\hline$D-34$ & $3 \mathrm{Ra}-228+\mathrm{D}$ & , milk/livestock-intake ratio, $(\mathrm{pCi} / \mathrm{L}) /(\mathrm{pCi} / \mathrm{d})$ & 3 & 1.000E-03 & 3 & $1.000 \mathrm{E}-03$ & 3 & $\operatorname{RTF}(12,3)$ \\
\hline$D-34$ & 3 & & 3 & & 3 & & 3 & \\
\hline$D-34$ & $3 \mathrm{Th}-228+\mathrm{D}$ & , plant/soil concentration ratio, dim & 3 & 1. $000 \mathrm{E}-03$ & 3 & 1. $.000 \mathrm{E}-03$ & 3 & $\operatorname{RTF}(13,1)$ \\
\hline$D-34$ & $3 T h-228+D$ & beef/livestock-intake ratio, $(\mathrm{pCi} / \mathrm{kg}) /(\mathrm{pCi} / \mathrm{d})$ & 3 & $1.000 E-04$ & 3 & $1.000 E-04$ & 3 & $\operatorname{RTF}(13,2)$ \\
\hline$D-34$ & $3 \mathrm{Th}-228+\mathrm{D}$ & , $\mathrm{milk} / \mathrm{livestock-intake} \mathrm{ratio,}(\mathrm{pCi} / \mathrm{L}) /(\mathrm{pCi} / \mathrm{d})$ & 3 & $5.000 \mathrm{E}-06$ & 3 & $5.000 E-06$ & 3 & $\operatorname{RTF}(13,3)$ \\
\hline$D-34$ & & & & & & & & \\
\hline
\end{tabular}


IRESRAD, Version $6.30 \quad T \ll$ Limit $=0.5$ year $\quad 11 / 01 / 2006 \quad 08: 58$ Page 4 Summary : 618-2 Staging Pile and Decontamination Pad Area RESRAD Evaluation File : 618-2 Staging Pile.RAD

Dose Conversion Factor (and Related) Parameter Summary (continued) File: HEAST 2001 Morbidity

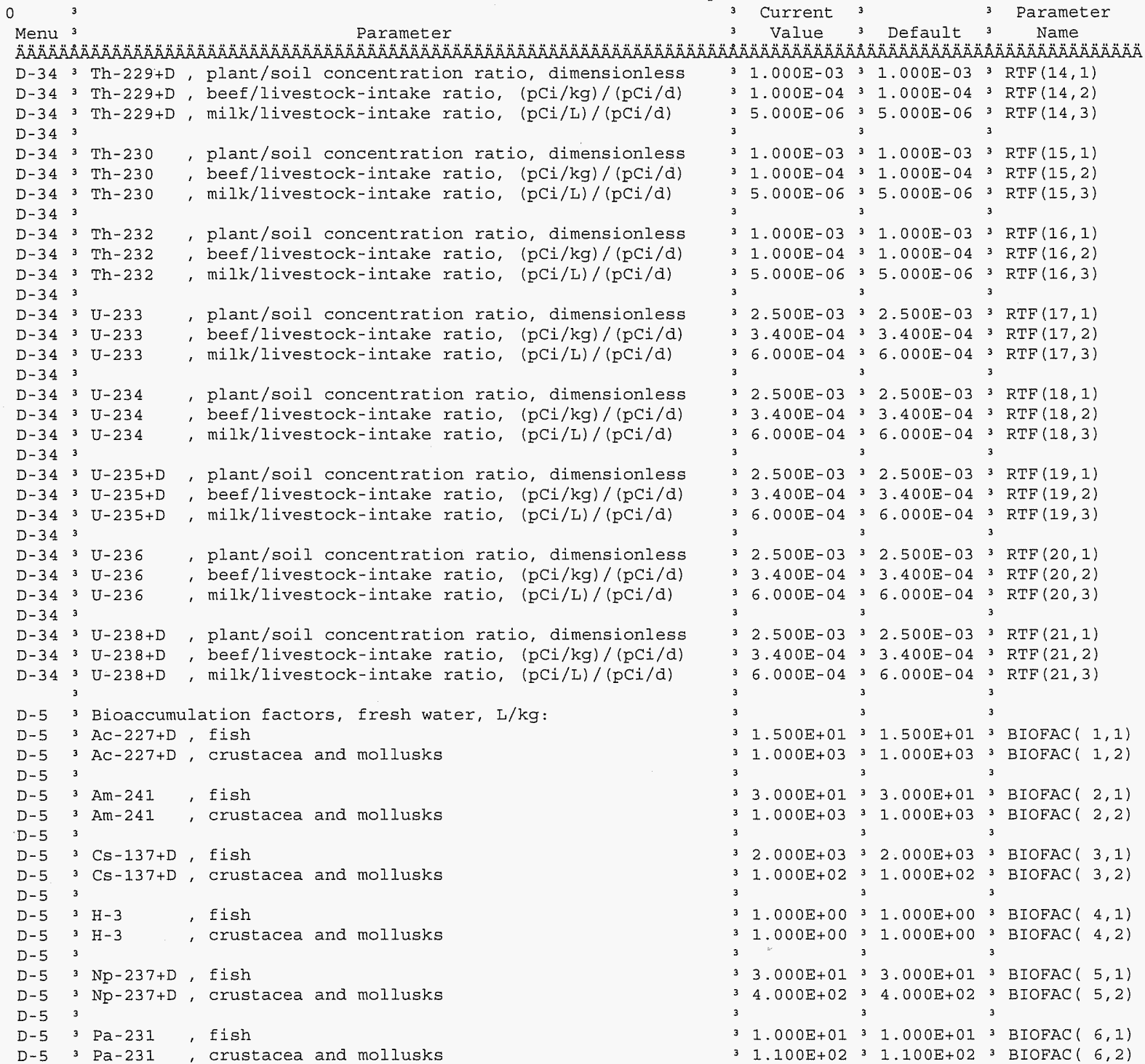


IRESRAD, Version $6.30 \quad T \ll$ Limit $=0.5$ year $\quad 11 / 01 / 2006 \quad 08: 58$ Page 5

Summary : 618-2 Staging Pile and Decontamination Pad Area RESRAD Evaluation

File : 618-2 staging Pile.RAD

Dose Conversion Factor (and Related) Parameter Summary (continued) File: HEAST 2001 Morbidity

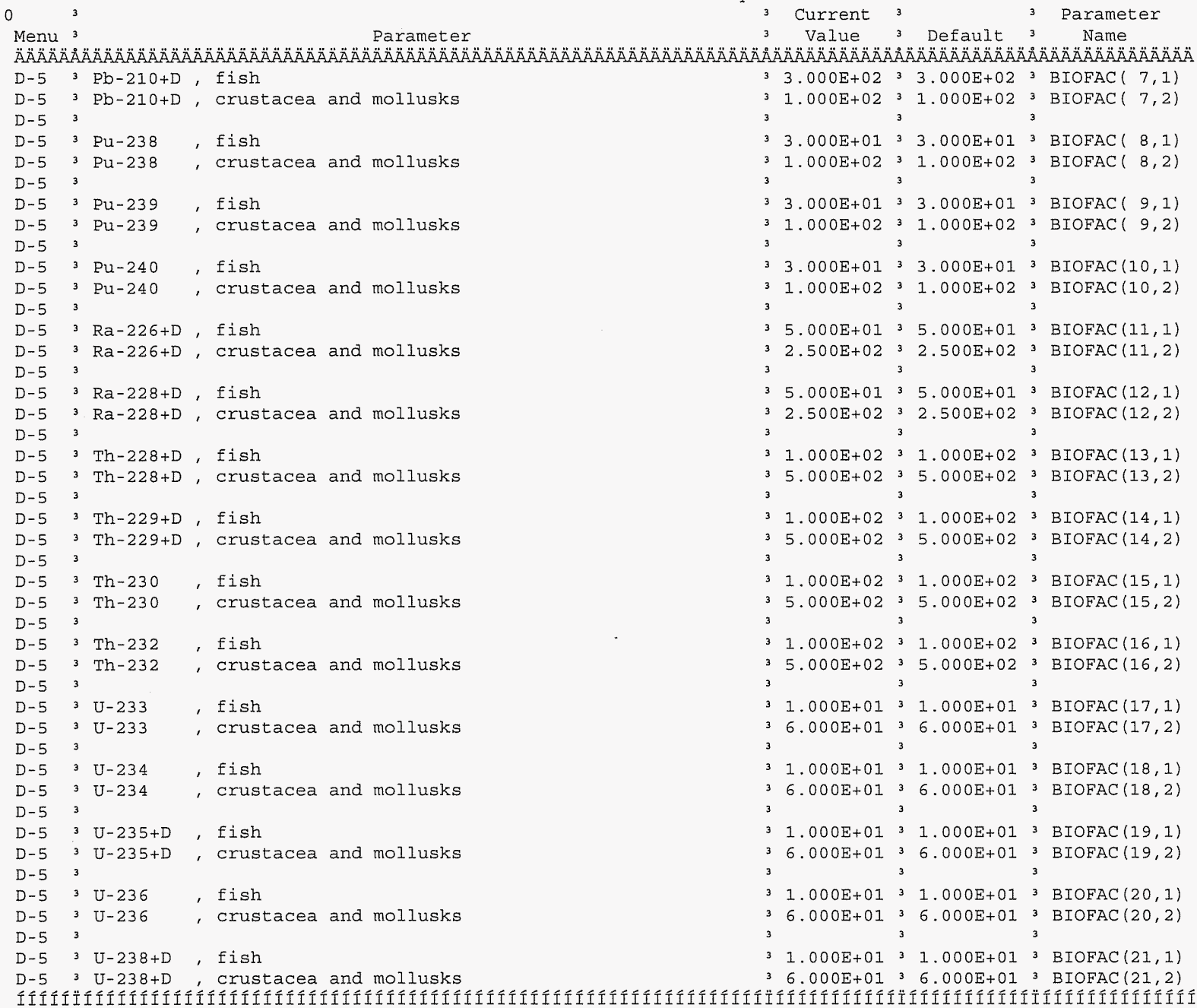


1RESRAD, Version $6.30 \quad$ T Limit $=0.5$ year $11 / 01 / 2006$ 08:58 Page
Summary : 618-2 Staging Pile and Decontamination Pad Area RESRAD Evaluation Summary : $618-2$ Staging Pile and
File : $618-2$ Staging Pile. RAD Site-Specific Parameter Summary Menu

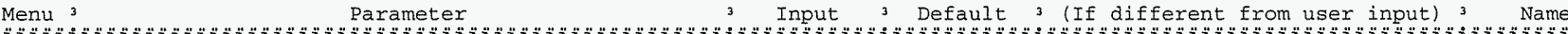
3 Used by RESRAD Used by RESRAD 3 Parameter

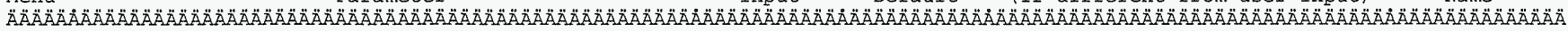
R011 3 Area of contaminated zone $(m * * 2)$ R011 3 Thickness of contaminated zone (m) R011 3 Length parallel to aquifer flow (m)

R011 3 Basic radiation dose limit (mrem/yr)

R011 3 Time since placement of material (yr)

R011 3 Times for calculations $(y x)$

R011 3 Times for calculations ( $\mathrm{Yr}$ )

R011 ${ }^{3}$ Times for calculations (yr)

R011 3 Times for calculations ( $y x$ )

R011 3 Times for calculations $(\mathrm{yr}$ )

R011 3 Times for calculations (yr)

R011 3 Times for calculations ( $\mathrm{yr}$ )

R011 3 Times for calculations ( $\mathrm{Yr}$ )

R011 3 Times for calculations ( $y r$ ) 3

R012 3 Initial principal radionuclide (pCi/g)

$\mathrm{R} 0123$ Initial principal radionuclide ( $\mathrm{pCi} / \mathrm{g}$ )

$\mathrm{R} 0123$ Initial principal radionuclide ( $\mathrm{pCi} / \mathrm{g}$ )

R012 3 Initial principal radionuclide ( $\mathrm{pCi} / \mathrm{g}$ )

$\mathrm{R} 012^{3}$ Initial principal radionuclide ( $\mathrm{pCi} / \mathrm{g}$ ):

R012 3 Initial principal radionuclide (pCi/g)

$\mathrm{R} 0123$ Initial principal radionuclide ( $\mathrm{pCi} / \mathrm{g}$ ):

$\mathrm{R} 0123$ Initial principal radionuclide $(\mathrm{pCi} / \mathrm{g})$ :

$\mathrm{R} 0123$ Initial principal radionuclide $(\mathrm{pCi} / \mathrm{g})$

R012 3 Concentration in groundwater

R012 3 Concentration in groundwater

$\mathrm{R} 0123$ Concentration in groundwater

R012 3 Concentration in groundwater

R012 3 Concentration in groundwater

R012 3 Concentration in groundwater

R012 3 Concentration in groundwater

R012 3 Concentration in groundwater

$(\mathrm{pCi} / \mathrm{L}):$

$(\mathrm{pCi} / \mathrm{L}):$

$(\mathrm{pCi} / \mathrm{L}):$

$32.071 \mathrm{E}+04 \quad 3 \quad 1.000 \mathrm{E}+04$

$3.600 \mathrm{E}+00,2.000 \mathrm{E}+00^{3}$

3 not used $31.000 E+02$

$31.500 \mathrm{E}+0132.500 \mathrm{E}+01$

$30.000 \mathrm{E}+003 \quad 0.000 \mathrm{E}+003$

$31.000 \mathrm{E}+00331.000 \mathrm{E}+00$

$33.000 \mathrm{E}+00 \quad 3 \quad 3.000 \mathrm{E}+003$

$31.000 E+0131.000 E+01$

$31.200 E+0133.000 E+01$

$33.800 \mathrm{E}+0131.000 \mathrm{E}+02$

$31.000 E+0233.000 E+023$

$3.000 \mathrm{E}+0231.000 \mathrm{E}+03$

$31.000 E+03 \quad 3 \quad 0.000 E+00$

3 not used $30.000 E+00^{3}$

$\mathrm{Am}-2413-1.500 \mathrm{E}-0133^{3} 0.000 \mathrm{E}+00$

Cs-137 3 $3.200 \mathrm{E}-02 \quad 3 \quad 0.000 \mathrm{E}+00$

\begin{tabular}{llll}
3 & $1.080 \mathrm{E}+00$ & 3 & $0.000 \mathrm{E}+00$ \\
\hline
\end{tabular}

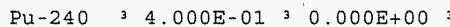

$\mathrm{U}-23433.390 \mathrm{E}-0130.000 \mathrm{E}+00$

$\mathrm{U}-23536.600 \mathrm{E}-03 \quad 0.000 \mathrm{E}+00$

$\begin{array}{lllll}U-238 & 3 & 2.940 \mathrm{E}-01 & 3 & 0.000 \mathrm{E}+00^{3}\end{array}$

3 not used $30.000 E+00$

3 not used $30.000 E+00{ }^{3}$

not used

not used $30.000 E+00$

$\mathrm{pCi} / \mathrm{L}): \mathrm{U}-2343$ not used $30.000 \mathrm{E}+00$

3 not used $30.000 E+00$

$(\mathrm{pCi} / \mathrm{T}) \cdot \mathrm{U}-238$

R013 ${ }^{3}$ Cover depth (m)

R013 3 Density of cover material $(\mathrm{g} / \mathrm{cm} * * 3)$

R013 3 Cover depth exosion rate $(\mathrm{m} / \mathrm{yr})$

R013 3 Density of contaminated zone $(\mathrm{g} / \mathrm{cm} * * 3)$

R013 3 Contaminated zone erosion rate $(\mathrm{m} / \mathrm{yr})$

R013 3 Contaminated zone total porosity

R013 3 Contaminated zone field capacity

R013 3 Contaminated zone hydraulic conductivity (m/yr)

R013 3 Contaminated zone b parameter

R013 3 Average annual wind speed $(\mathrm{m} / \mathrm{sec})$

R013 3 Humidity in air $(\mathrm{g} / \mathrm{m} * * 3)$

R013 3 Evapotranspiration coefficient

R013 3 Precipitation ( $\mathrm{m} / \mathrm{yr}$ )

R013 3 Irrigation $(\mathrm{m} / \mathrm{yr})$

R013 3 Irrigation mode

R013 3 Runoff coefficient

${ }_{3}^{3}$ not used ${ }_{3}^{3} 0.000 \mathrm{E}+00_{3}^{3}$

$30.000 E+00^{3} \quad 0.000 E+00$

3 not used $31.500 \mathrm{E}+00$

not used $31.000 \mathrm{E}-03$

$31.600 \mathrm{E}+0031.500 \mathrm{E}+00$

$3.000 E-0331.000 E-03$

$3.000 \mathrm{E}-0134.000 \mathrm{E}-01$.

$2.500 E-0132.000 E-01$

$2.200 \mathrm{E}-03$ 3 $1.000 \mathrm{E}+01$

$31.500 \mathrm{E}+0135.300 \mathrm{E}+00$

$33.400 E+0032.000 E+00$

$38.000 \mathrm{E}+0038.000 \mathrm{E}+00$

$39.100 \mathrm{E}-0135.000 \mathrm{E}-01$

$31.524 \mathrm{E}-0131.000 \mathrm{E}+00$

${ }^{3} 7.600 \mathrm{E}-0132.000 \mathrm{E}-01$

3 overhead 3 overhead 3

32.000 E-0132.000E-013

3 AREA

-. $\quad 3$ THICKO

-.. 3 LCZPAQ

3 BRD

-.- 3 TI

-.- $3 \mathrm{~T}(2)$

3 T( 3$)$

3
3 $(4)$

${ }^{3} \mathrm{~T}(5)$

-.- $3 \mathrm{~T}(6)$

-. $3 \mathrm{~T}(7)$

$3 T(8)$

$\begin{array}{ll}3 & T(9) \\ 3 & T(10)\end{array}$

$-\cdots$

-- $\quad 3$ S1 ( 3 )

${ }^{3} \operatorname{SI}(4)$

${ }^{3} \operatorname{Sil}(8)$

3 SI 9 )

$3 \mathrm{SI}(10)$

-.- $\quad 3$ SI (18)

${ }^{3} S 1(19)$

$\begin{array}{ll}3 & S 1(21) \\ 3 & \text { W1 }\end{array}$

3 W $1(3)$

$--$

3 WI $(8)$

-.. 3 WI $(9)$

3 WI (10)

${ }^{3}$ W1 (18)

${ }_{3}$ WI (19)

$-\cdots$

-. 3 COVERO

3 VCV

--- 3 DENSCZ

-.- $3 \mathrm{VCZ}$

-.

3 FCCZ

${ }^{3} \mathrm{HCCZ}$

${ }^{3} \mathrm{BCZ}$

-.- 3 WIND

3 HUMID

3 EVAPTR

3 RI

${ }^{3}$ IDITCH

3 RUNOFF 


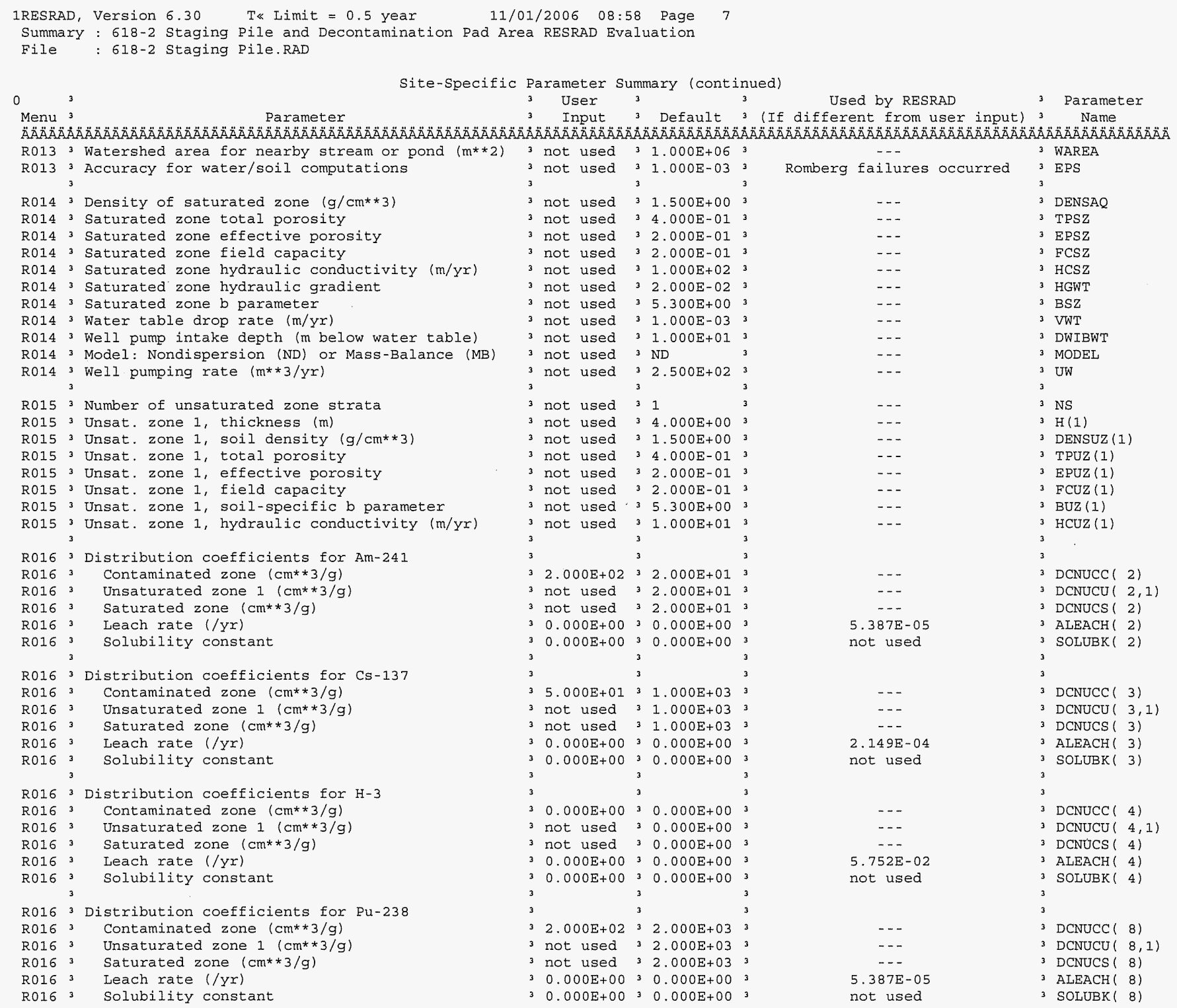




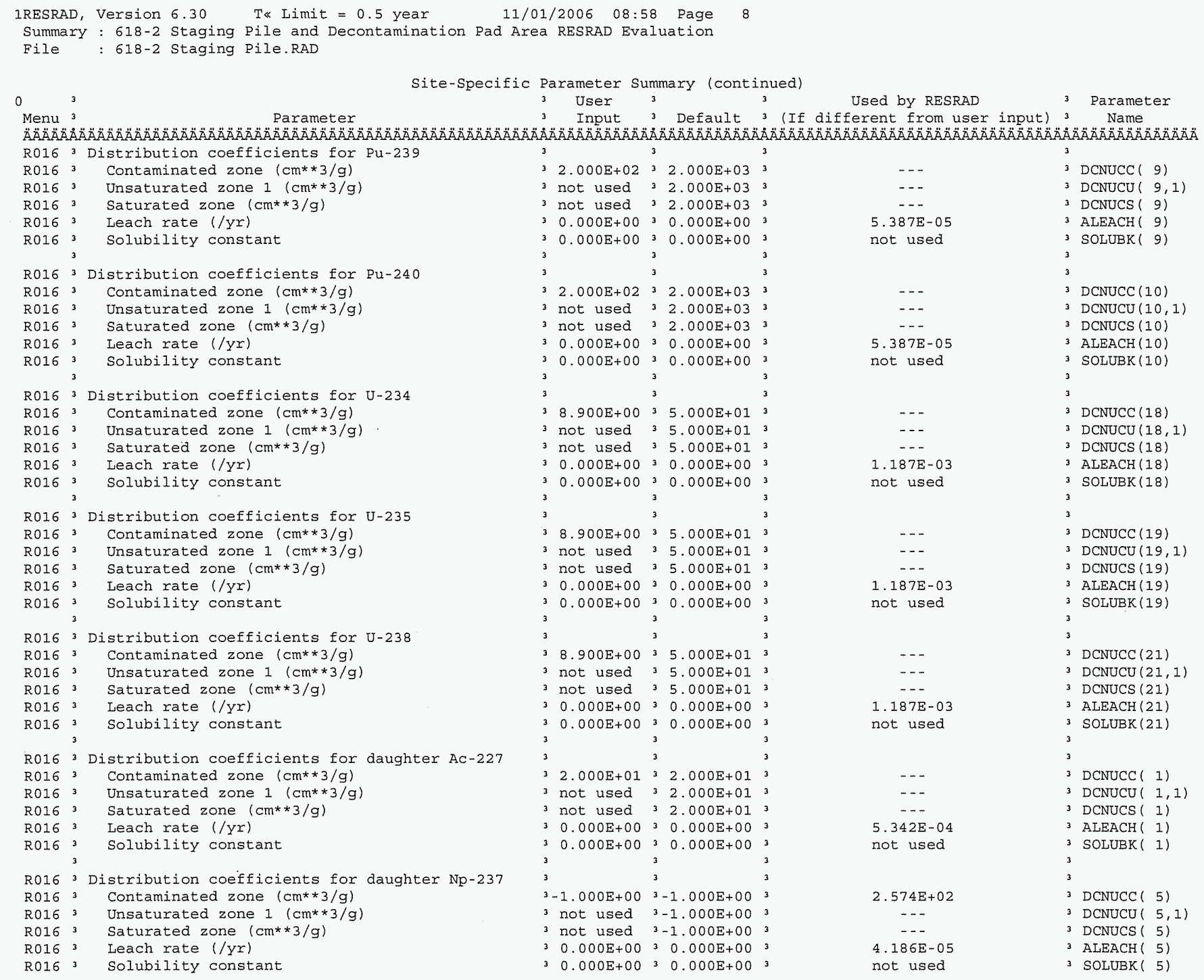


IRESRAD, Version $6.30 \quad$ T* Limit $=0.5$ year $11 / 01 / 2006 \quad 08: 58$ Page 9

Summary : 618-2 Staging Pile and Decontamination Pad Area RESRAD Evaluation

File: $618-2$ staging Pile.RAD

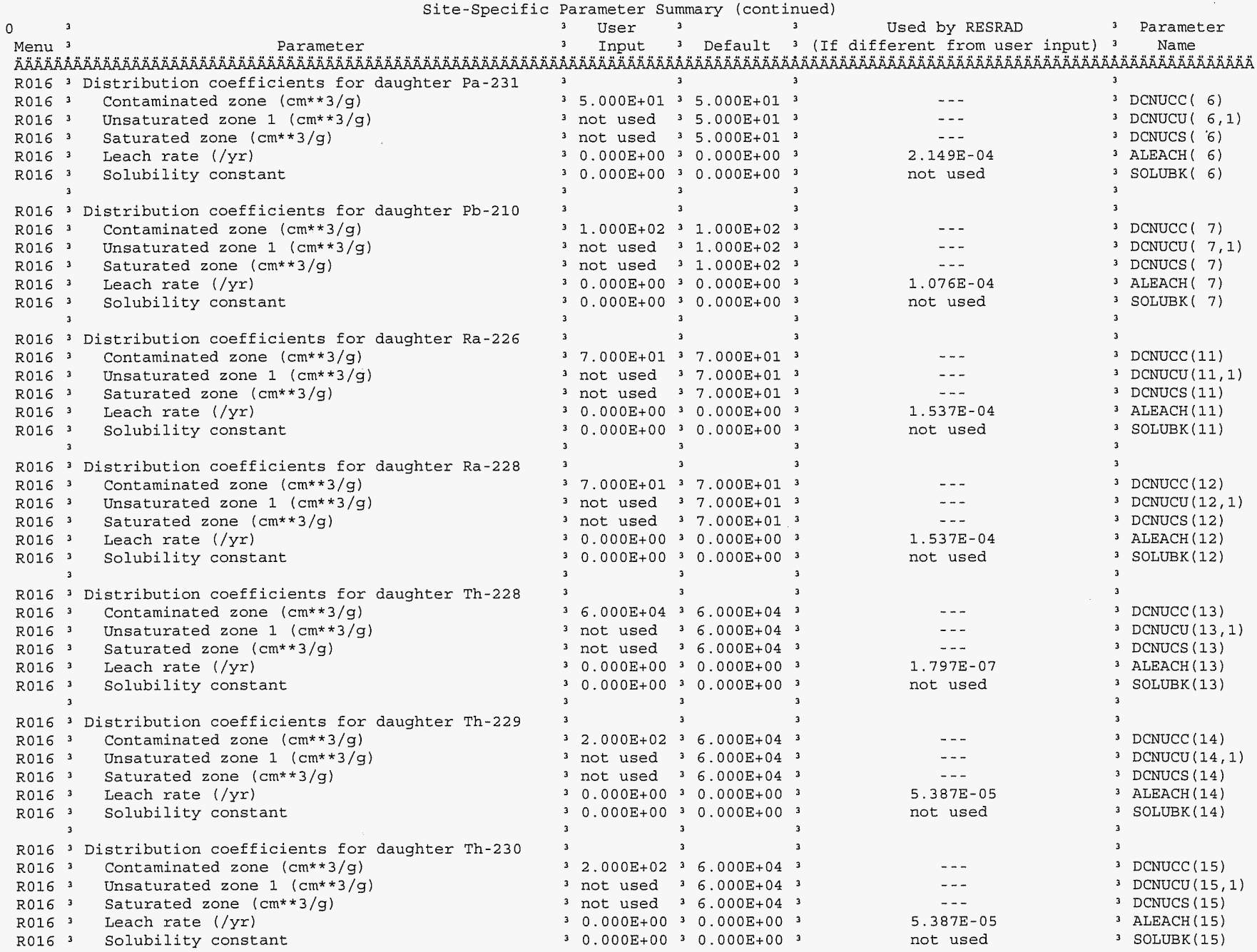


IRESRAD, Version $6.30 \quad$ T\& Limit $=0.5$ year $\quad 11 / 01 / 2006 \quad 08: 58$ Page 10 Summary : 618-2 Staging Pile and Decontamination Pad Area RESRAD Evaluation File : 618-2 staging Pile.RAD

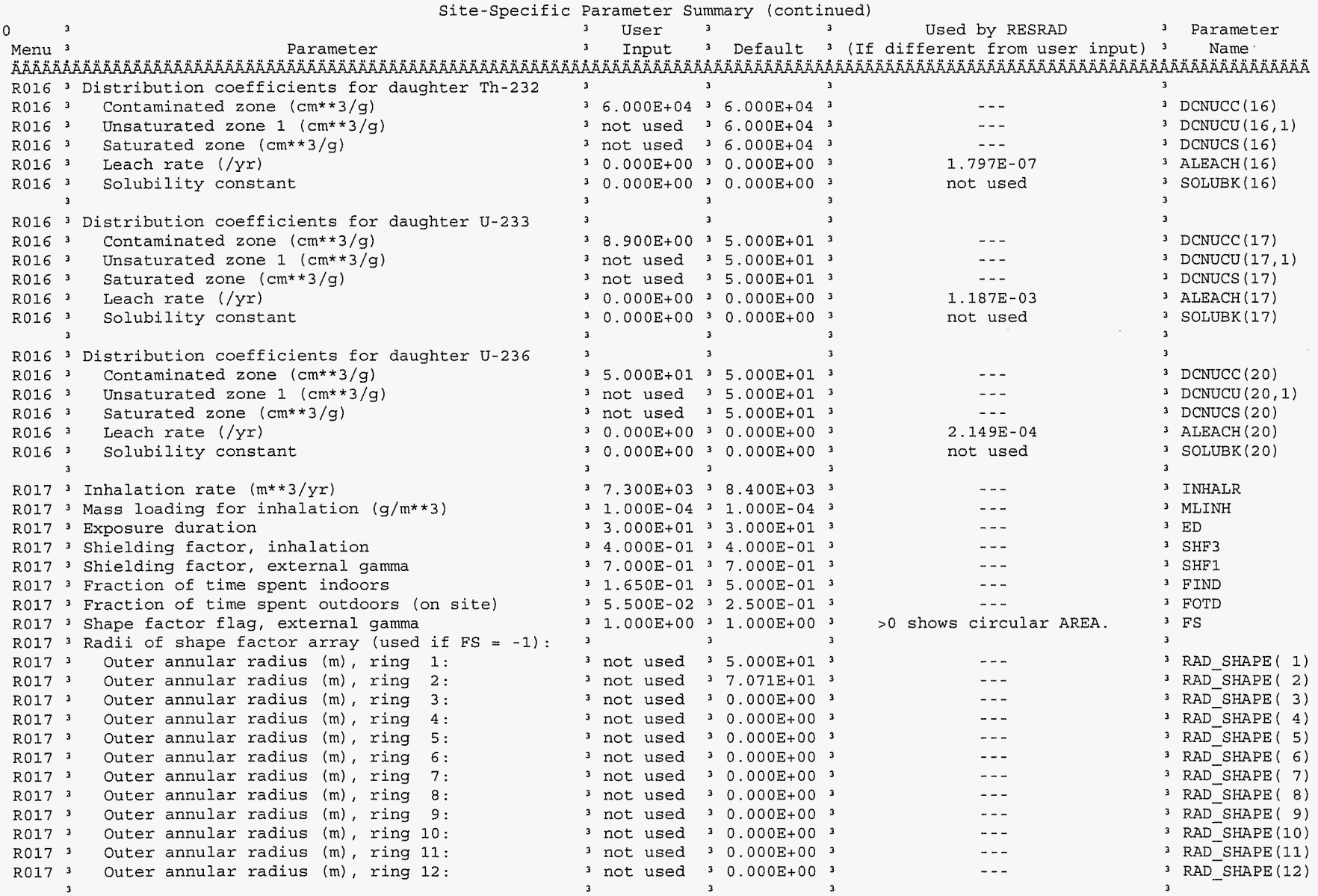


IRESRAD, Version $6.30 \quad T$ Limit $=0.5$ year $\quad 11 / 01 / 2006 \quad 08: 58$ Page 11 Summary : 618-2 Staging Pile and Decontamination Pad Area RESRAD Evaluation File : 618-2 staging Pile.RAD

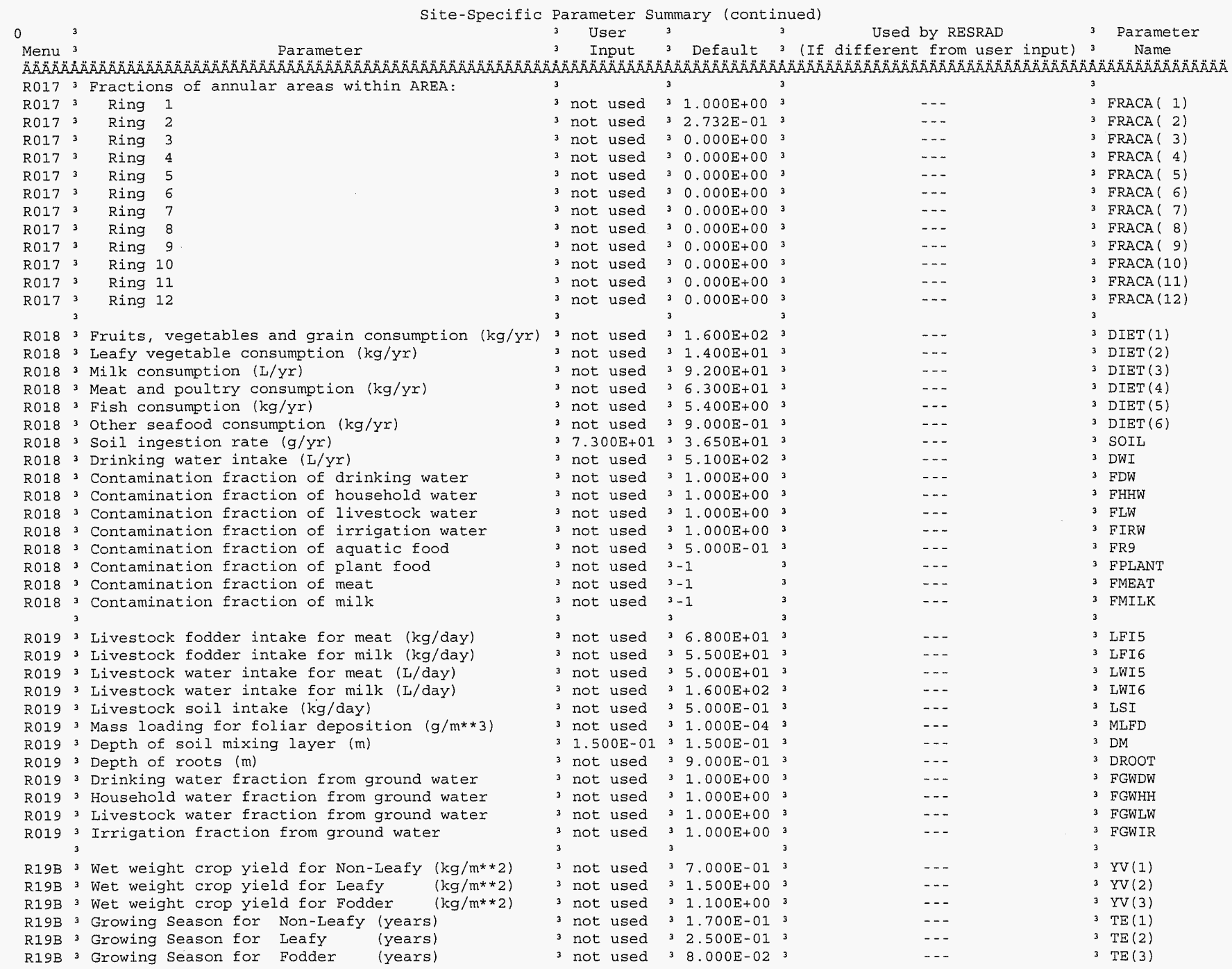


IRESRAD, Version $6.30 \quad$ T\& Limit $=0.5$ year $11 / 01 / 2006 \quad 08: 58$ Page 12

Summary : 618-2 Staging Pile and Decontamination Pad Area RESRAD Evaluation

File : 618-2 staging Pile.RAD

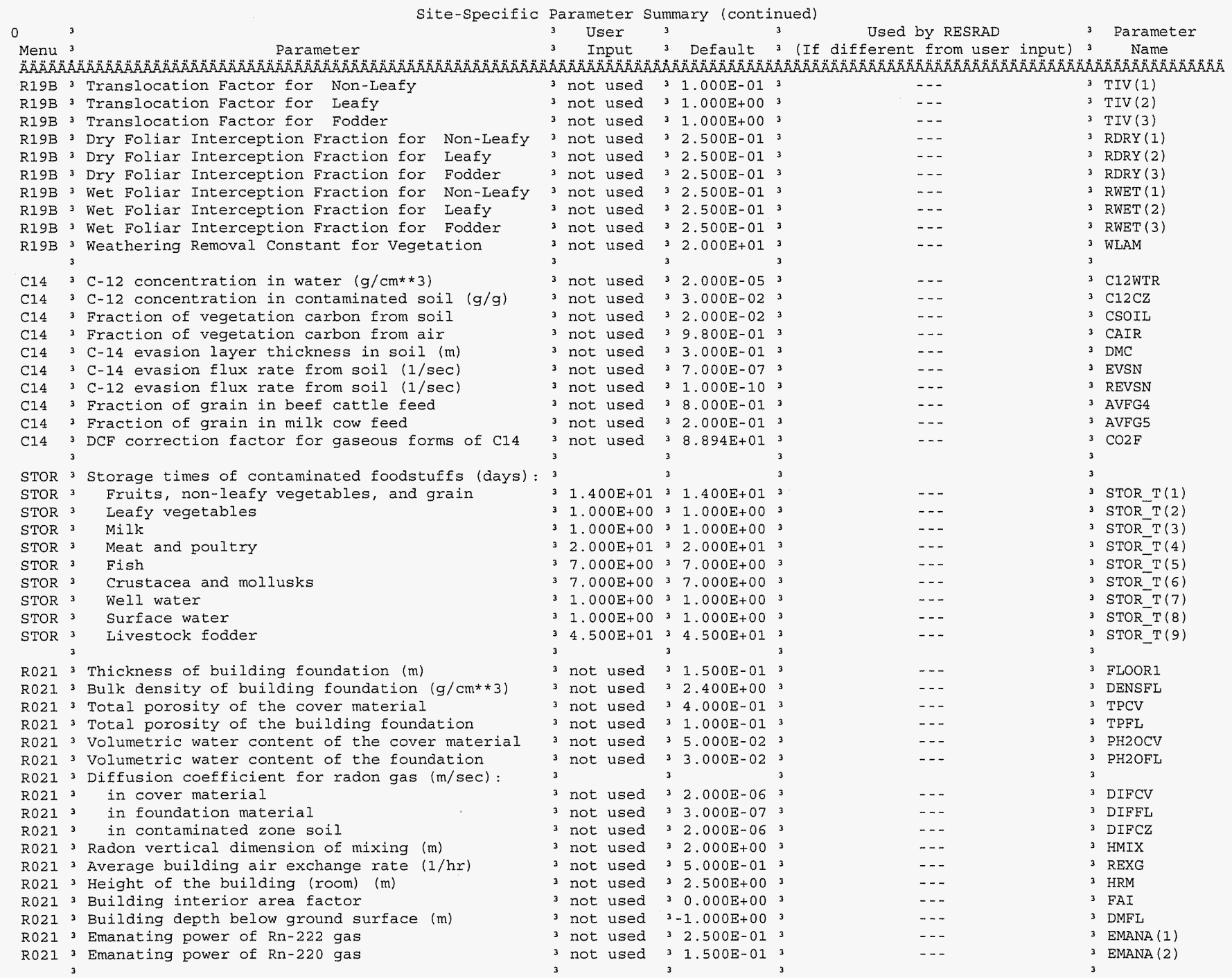


IRESRAD, Version $6.30 \quad$ T\& Limit $=0.5$ year 11/01/2006 08:58 Page 13

Summary : 618-2 Staging Pile and Decontamination Pad Area RESRAD Evaluation

File: 618-2 staging Pile.RAD

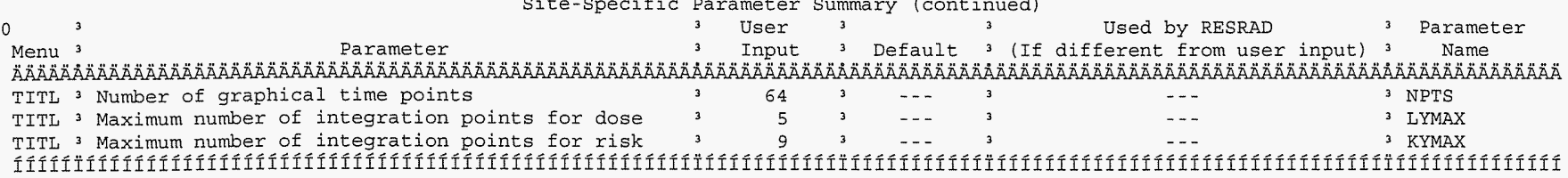

Summary of Pathway Selections

Pathway User Selection

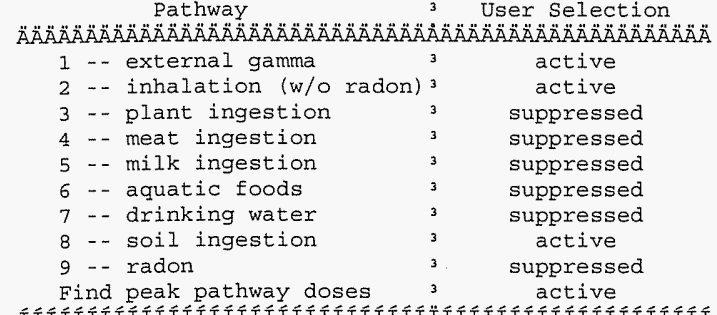

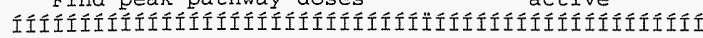




\section{DISTRIBUTION}

U.S. Department of Energy

Richland Operations Office

S. L. Sedgwick

A3-04

D. C. Smith (5)

A3-04

$\mathrm{RL}$ Correspondence Control

A5-10

DOE-RL Public Reading Room

$\mathrm{H} 2-53$

Fluor Hanford, Inc.

J. P. Shearer (WIDS)

A0-21

Washington Closure Hanford

K. A. Anselm

$\mathrm{H} 4-23$

R. A. Carlson

X4-08

S. W. Clark

$\mathrm{H} 4-23$

F. M. Corpuz

L6-06

J.W. Darby

L6-06

L. A. Dietz

H4-22

L. M. Dittmer

$\mathrm{H} 4-23$

M. J. Haass

L6-06

R. B. Kerkow

L6-06

J. E. Thomson

$\mathrm{H} 4-22$

W. S. Thompson

$\mathrm{H} 4-23$

Records and Document Control

H4-11

Hanford Technical Library

P8-55 
CVP-2006-00010

Rev. 0

Distr-2 